\title{
From vulnerability to resilience against depression : the value of daily-life positive emotions
}

Citation for published version (APA):

Geschwind, N. (2011). From vulnerability to resilience against depression : the value of daily-life positive emotions. [Doctoral Thesis, Maastricht University]. Maastricht University. https://doi.org/10.26481/dis.20111207ng

Document status and date:

Published: 01/01/2011

DOI:

10.26481/dis.20111207ng

Document Version:

Publisher's PDF, also known as Version of record

\section{Please check the document version of this publication:}

- A submitted manuscript is the version of the article upon submission and before peer-review. There can be important differences between the submitted version and the official published version of record.

People interested in the research are advised to contact the author for the final version of the publication, or visit the DOI to the publisher's website.

- The final author version and the galley proof are versions of the publication after peer review.

- The final published version features the final layout of the paper including the volume, issue and page numbers.

Link to publication

\footnotetext{
General rights rights.

- You may freely distribute the URL identifying the publication in the public portal. please follow below link for the End User Agreement:

www.umlib.nl/taverne-license

Take down policy

If you believe that this document breaches copyright please contact us at:

repository@maastrichtuniversity.nl

providing details and we will investigate your claim.
}

Copyright and moral rights for the publications made accessible in the public portal are retained by the authors and/or other copyright owners and it is a condition of accessing publications that users recognise and abide by the legal requirements associated with these

- Users may download and print one copy of any publication from the public portal for the purpose of private study or research.

- You may not further distribute the material or use it for any profit-making activity or commercial gain

If the publication is distributed under the terms of Article $25 \mathrm{fa}$ of the Dutch Copyright Act, indicated by the "Taverne" license above, 


\section{From vulnerability to resilience against depression}

\section{The value of daily-life positive emotions}

Nicole Geschwind 
ISBN 978-90-8570-630-4

Cover Design: Nicole Geschwind, with thanks to Edward Monkton for letting me use his "Elephant of joy" Printing: Wöhrmann Print Service Layout: Nicole Geschwind

Copyright (C) Nicole Geschwind, Maastricht 2011 All rights reserved. No part of this publication may be reproduced, stored in a retrieval system, or transmitted, in any form or by any means, without the prior permission from the proprietor. 


\title{
From vulnerability to resilience against depression
}

\section{The value of daily-life positive emotions}

\author{
PROEFSCHRIFT
}

Ter verkrijging van de graad van doctor aan de Universiteit Maastricht, op gezag van

de Rector Magnificus, Prof. mr. G.P.M.F. Mols, volgens het besluit van het College van Decanen, in het openbaar te verdedigen op woensdag 7 december 2011 om 12 uur

door

Nicole Mary Geschwind

Geboren op 7 september 1979 te Bremen, Duitsland 


\section{Promotor}

Prof. dr. J. van Os

\section{Co-promotores}

Dr. M. Wichers

Dr. F. Peeters

\section{Beoordelingscommissie}

Prof. dr. I. Myin-Germeys (voorzitter)

Prof. dr. A. Beekman (Vrije Universiteit Amsterdam)

Prof. dr. M. Peters

Dr. F. Raes (Katholieke Universiteit Leuven, België)

Dr. K. Schruers

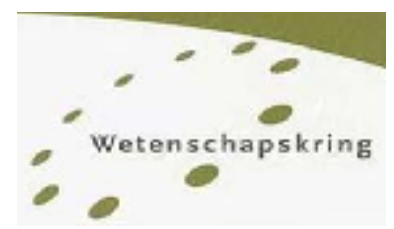

South Limburg Mental Health and Teaching Network PhD Series

The research presented in this thesis was conducted at the School for Mental Health and Neuroscience, Department of Psychiatry and Psychology of Maastricht University, Maastricht University Medical Centre.

Publication of this thesis was financially supported by Eli Lilly Nederland B.V., Servier Nederland Farma B.V., and Lundbeck B.V. 
To Sibren and Jamie

The Elephant of Joy

Let us, like the Elephant, Live happy and live true

By finding JOY and WONDER In each little thing we do.

Edward Monkton 
Paranimfen

Rufa Diederen

Nicole Leibold 


\section{CONTENTS}

Chapter 1

General Introduction

Chapter 2

The role of affective processing in vulnerability to and resilience against depression

Chapter 3

Scars in depression: Is a conceptual shift necessary to solve the puzzle?

\section{Chapter 4}

Transition from stress sensitivity to a depressive state: A longitudinal twin study

Chapter 5

Early improvement in positive rather than negative emotion predicts remission from depression after pharmacotherapy

\section{Chapter 6}

Meeting risk with resilience: High daily-life reward experience preserves mental health

Chapter 7

Mindfulness training increases momentary positive emotions and reward experience in adults vulnerable to depression. A randomized controlled trial

Chapter 8

Mindfulness-based cognitive therapy reduces residual depressive symptoms irrespective of number of prior depressive episodes. A randomized controlled trial

Chapter 9

General Discussion

Summary

Samenvatting

References

C.V. and Publications 



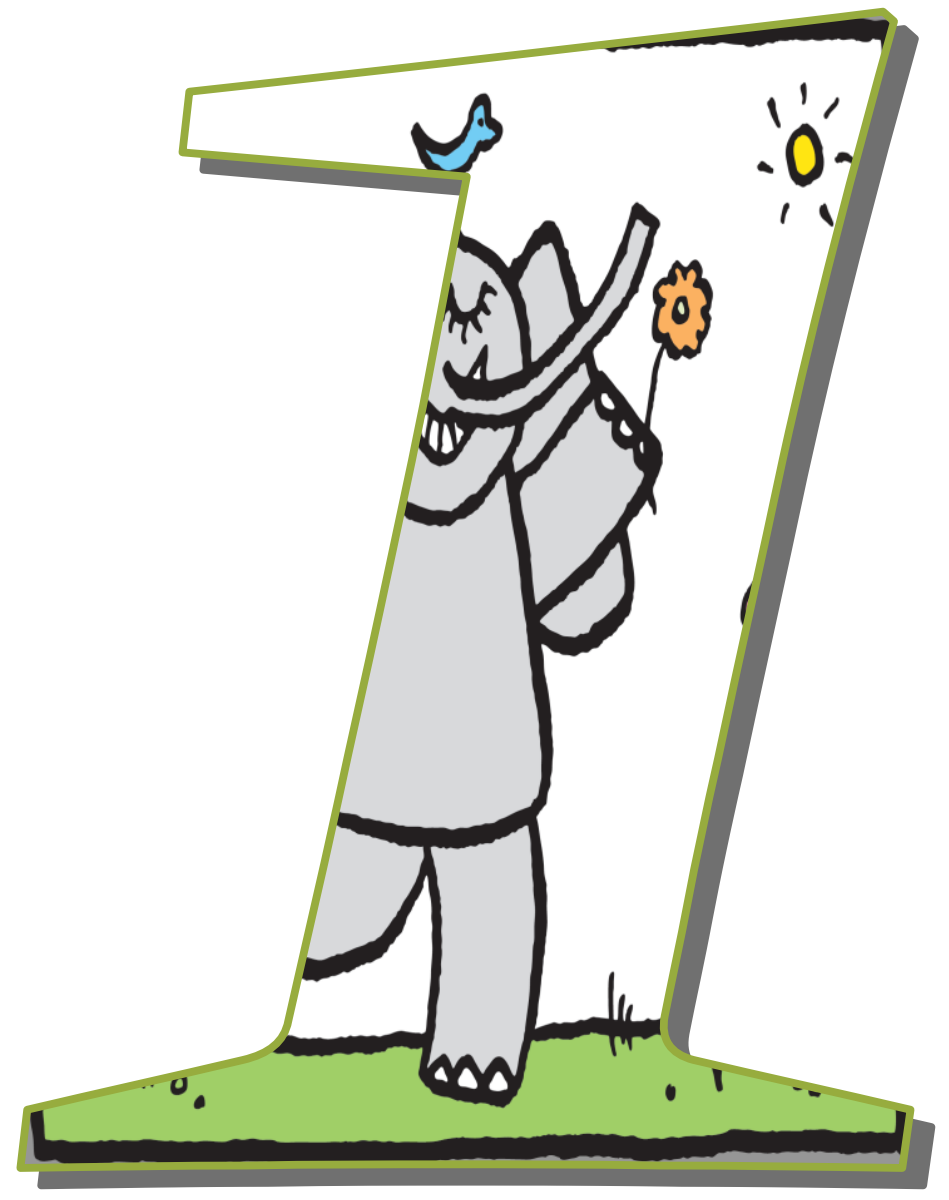

\section{General Introduction}




\section{DEPRESSION}

Depressive disorders represent an enormous burden for health. According to the World Health Organization (WHO; 2001), depression was the largest cause of disability in the year 2000, and fourth on the list of contributors to the global burden of disease. By 2020, the WHO predicts depression to have become the second leading contributor to burden of disease. Depression thus is a common and disabling mental disorder.

Symptoms include a combination of miserable mood, loss of interest or pleasure, feelings of guilt or low self-worth, disturbed sleep or appetite, low energy, and poor concentration. Virtually everybody has experienced some symptoms of depression, especially after exposure to stressful life events or chronic stress. If five out of nine symptoms of depression are present for longer than two weeks, and if these symptoms result in impairment and loss of functioning, the criteria for a major depressive episode are met (American Psychiatric Association, 2000). Depressed mood and loss of interest or pleasure are seen as core symptoms; therefore one of these two symptoms has to be present for diagnosing a major depressive episode. The WHO (2001) estimates that world-wide about $10 \%$ of women and $6 \%$ of men experience a depressive episode per year, although prevalence estimates of Major Depression vary widely across populations.

While a categorical approach (i.e., depression present or absent) may be useful in clinical settings, cut-off points and exact criteria for diagnosing a major depressive episode are largely arbitrary. There are strong arguments for adapting a dimensional approach in which depressive symptoms wax and wane, varying on a continuum from absent to very impairing. For example, subthreshold depressive symptoms predict increased risk for future major depressive episodes (Cuijpers \& Smit, 2004), and are associated with considerable impairment in functioning (Judd, Schettler, \& Akiskal, 2002). Therefore, we generally adapted the dimensional approach throughout this thesis.

\section{VULNERABILITY AND RESILIENCE}

In the past, research has largely focused on what makes people vulnerable to psychopathology (Seligman \& Csikszentmihalyi, 2000). With the advent of the positive psychology movement, researchers have started to show more interest in protective influences (Gable \& Haidt, 2005). While some people show remarkable resilience to depression, others are particularly vulnerable. Studying both vulnerability and resilience will provide a more complete picture of how to 
prevent or treat depression and enhance well-being. What is it that makes someone vulnerable? What behavior or traits do resilient people possess? And how do vulnerability and resilience interact? Can we teach vulnerable individuals to adapt characteristics of resilient people? These questions are addressed in the current thesis. Obviously, processes of vulnerability and resilience occur in various domains such as emotion ${ }^{1}$, perception, cognition, social support and coping. The scope of this thesis is limited to the affective domain.

\section{POSITIVE AND NEGATIVE AFFECT}

Investigations into the factor structure of emotion have converged on two-factor models, commonly labeled positive and negative affect (PA and NA; Almagor \& Ben-Porath, 1989; Watson \& Tellegen, 1985). PA is associated with behavioral approach and characterized by such feelings as enthusiasm, interest and happiness. NA is associated with behavioral withdrawal and includes such feelings as distress, sadness and hostility. In depression, the processing of both NA and PA appears to be altered (Leppänen, 2006). As mentioned earlier, increased NA as well as a reduced capacity to experience PA (anhedonia) are core symptoms of depression (American Psychiatric Association 2000).

Several findings suggests that PA and NA are related yet independent systems (Watson, Wiese, Vaidya, \& Tellegen, 1999). First of all, PA and NA have different functions. NA is usually linked to short-term action tendencies (Frijda, Kuipers, \& ter Schure, 1989; Tooby \& Cosmides, 1990). Fear, for example, motivates the urge to escape, and anger motivates the urge to attack. PA, on the other hand, likely has more general and long-term functions. According to the Broaden-and-Build Theory, PA encourages exploration of opportunities and interaction with others, which on the long run will strengthen social bonds (Fredrickson, 2001, 2004). Second, PA and NA have different diurnal patterns. While NA is relatively independent of the time of day, PA is low in the morning, peaks over day and is low before going to bed (Clark, Watson, \& Leeka, 1989). Third, in-the-moment measurements of PA and NA reveal only moderate negative correlations (though the strength of correlation varies with the intensity of emotions and is stronger at very intense levels of emotion) (Diener \& Emmons, 1984).

\footnotetext{
${ }^{1}$ Although the terms affect and emotion are sometimes distinguished, definitions vary. Because the current thesis did not did not investigate particular emotions but clusters of positive and negative emotions (positive affect and negative affect), we have used the terms affect and emotion interchangingly.
} 
Levels of NA and PA vary from moment to moment, as illustrated in Figure 1. Everyday experiences are a major source of moment-to-moment variation. For example, an unexpected smile can make someone feel elated, whereas a careless word can make someone feel upset or angry. The extent to which emotions are influenced by everyday situations varies between individuals. Some people are very sensitive to stress; they react to unpleasant daily-life situations with a much larger increase in NA than others. Similarly, some people are more sensitive to rewarding situations - in other words, they generate more PA from pleasant events or activities than others (a tendency hereafter referred to as high reward experience).

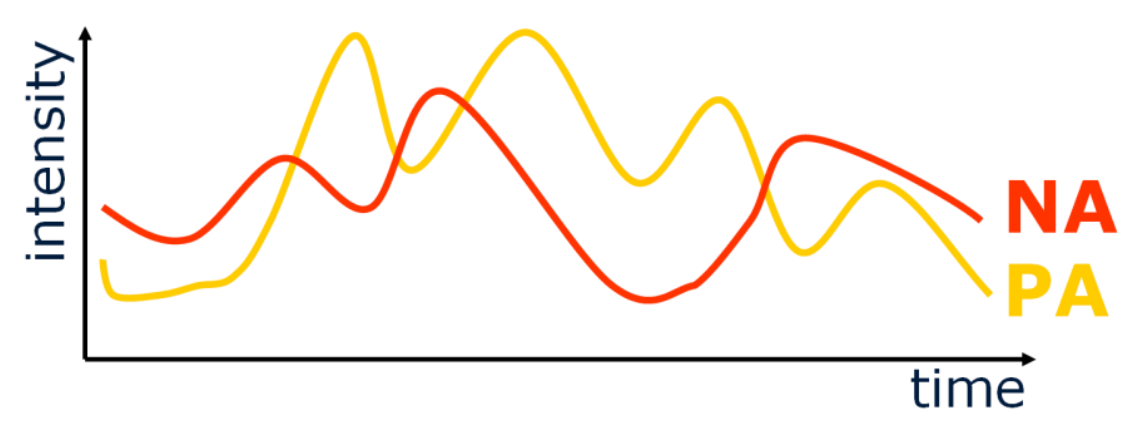

Figure 1. NA and PA vary from moment to moment, depending on experiences in the flow of daily life. Retrospective assessment methods do not measure this variability very well. NA $=$ Negative Affect, PA = Positive Affect

Tendencies to react to daily-life situations in a certain way do not only have short-term (in terms of e.g. momentarily altered emotional state) but also longterm consequences. For example, heightened daily-life stress sensitivity appears to be a risk indicator for depression (Jacobs, et al., 2006; Wichers, et al., 2007b). On the other hand, high reward experience as well as the tendency to experience high overall levels of positive emotion appear to be protective (e.g., Cohn, Fredrickson, Brown, Mikels, \& Conway, 2009; Danner, Snowdon, \& Friesen, 2001; Fredrickson, 2001; Garland, et al., 2010; Wichers, et al., 2010). How these everyday affective response patterns interact with risk for depression, resulting in increased vulnerability to or resilience against depression, is the topic of this thesis.

Affective responses to daily-life situations are difficult to measure with conventional methods such as retrospective questionnaire or interview. The Experience Sampling Method (ESM) captures emotional variability in people's 
own daily-life context and is the method of choice for a rigorous assessment of emotions in daily life. Therefore, ESM was used in nearly all studies reported in this thesis.

\section{THE EXPERIENCE SAMPLING METHOD}

ESM is a momentary assessment method to assess participants in their daily living environment, thus providing repeated in-the-moment assessments of affect in a prospective and ecologically valid manner (Csikszentmihalyi \& Larson, 1987; De Vries, 1992; Peeters, Nicolson, Berkhof, Delespaul, \& De Vries, 2003). Compared to retrospective questionnaires and interviews, ESM offers several advantages: (a) enhanced ecological validity, because participants are assessed in their normal daily environment, (b) minimized retrospective bias, because participants' experiences are assessed in the moment, and (c) enhanced reliability, because participants' experiences are assessed repeatedly (Csikszentmihalyi \& Larson, 1987). Apart from the sensitive measurement of concurrent emotions, ESM is ideally suited to assess person-context interactions in daily life, such as stress sensitivity and reward experience, as shown in Figure 2.

In the studies used for the present thesis, participants received a digital wristwatch and a set of ESM self-assessment forms collated in a booklet for each day. The wristwatch emitted a signal ("beep") at ten preprogrammed unpredictable moments during each day. Assessment periods usually lasted five to six days, thus resulting in a maximum of 60 beeps per study period. After each beep, participants were asked to complete their appraisals of current thoughts, mood, and context on 7-point Likert scales. 


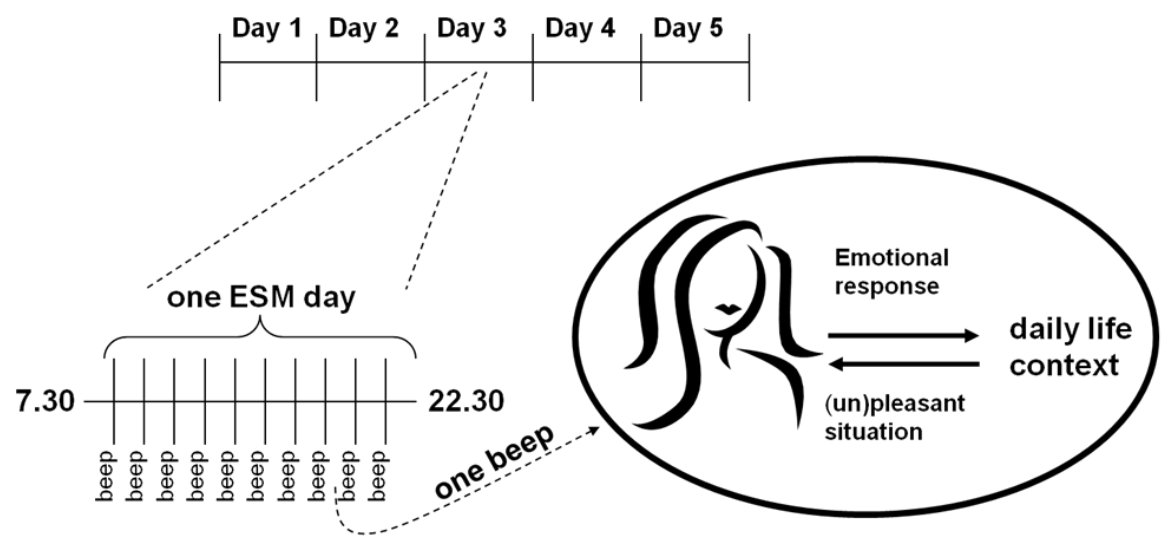

Figure 2. The Experience Sampling Method procedure and its potential to measure person-context interactions. Participants complete up to ten assessments of in-the-moment emotion and context per day. Affective response patterns such as stress sensitivity (the tendency to experience high NA reactivity in response to unpleasant situations) or reward experience (the tendency to experience high PA reactivity in response to pleasant situations) can then be determined. $E S M=$ Experience Sampling Method; NA = Negative Affect, PA = Positive Affect

\section{FROM ETIOLOGY TO INTERVENTION}

In a nutshell, this thesis presents experience sampling research ${ }^{2}$ on the role of daily-life affective processing in vulnerability to and resilience against depressive symptoms. Vulnerability and resilience are approached from a dimensional view, focusing on symptom expression rather than using diagnostic categories.

We first discuss the role of daily-life emotions and person-context interactions in the etiology of depression. More specifically, how are daily-life positive and negative emotions and the related affective constructs reward experience and stress sensitivity involved in the development of depressive symptoms? Second, we investigate the role of positive and negative emotions in the prediction of recovery from depression. Finally, based on the outcome of these studies, we move on to the level of intervention and present a new way to increase resilience against depressive symptoms.

\footnotetext{
${ }^{2}$ With the exception of Chapter 8 (which is based on interview and questionnaire data).
} 


\section{OUTLINE OF MY THESIS}

Chapter 2 discusses the role of affective processing in vulnerability to and resilience against depression. Chapter 2 can be seen as an extension to the General Introduction. Several examples illustrate the complex nature of the diagnostic concept "depression", with interacting genetic and environmental influences. The advantages of examining more proximal intermediate phenotypes, rather than diagnostic categories, are discussed, as well as the potential of ESM to contribute to valid measurements of such intermediate phenotypes. The chapter closes by highlighting the need for further research on the role of positive emotions in resilience against depressive symptoms.

Chapters 3 and 4 are concerned with vulnerability to depression. Chapter 3 is a conceptual article which outlines a new approach in the examination of scar theories. Scar theories have evolved in an attempt to explain the finding that the chances on future episodes of major depression increase as a function of the number of earlier episodes. Scar theories assume that changes during an episode of depression induce a long-lasting change and thereby increase the likelihood of future episodes. Nevertheless, empirical research on post-depressive scarring has largely remained inconclusive. In Chapter 4, the issue of vulnerability is further explored in a longitudinal twin study. We examined associations between baseline daily-life stress sensitivity and later depressive state, and identified genetic and non-genetic factors moderating the transition from stress sensitivity to depression.

Chapters 5 to 7 deal with resilience against and recovery from depression, focusing in particular on the role of daily-life positive emotions. In Chapter 5, we examined whether monitoring early change in positive and negative emotion during the first week of pharmacotherapy might improve clinical decision making. Current strategies for finding the right antidepressant for an individual include trial-and-error. Enriching the clinical decision-making process with accurate outcome predictors at an early change of treatment therefore has the potential to considerably improve quality of life for depressed patients. Studies have implicated the activation of resilience-like mechanisms in recovery from depression and response to pharmacotherapy. Other research suggests that positive rather than negative emotions predict resilience. Yet conventional instruments for scoring improvement hardly take positive emotions into account. We therefore expected early change in positive emotion to be an important predictor of response and remission, and to contribute over and above outcome prediction using conventional instruments. 
Chapter 6 presents the results of a longitudinal study on daily-life reward experience. Given the existing literature which demonstrates that the experience of high levels of positive emotions preserves and possibly augments mental health, high reward experience (i.e., the ability to generate boosts in PA from pleasant daily-life situations) should be beneficial. If high reward experience was indeed associated with preserved mental health, this would point to possible interventions aimed at generating more positive emotions in daily life: Teaching people to make more efficient use of pleasant daily-life situations. We therefore examined prospectively whether high daily-life reward experience was associated with increased resilience against affective symptoms, and whether environmental or genetic risk factors moderated these potential effects.

In Chapter 7, we present a randomized controlled trial on the potential of Mindfulness-Based Cognitive Therapy (MBCT) to increase emotional resilience against depression. MBCT is a group skills-training program which combines meditation exercises (Kabat-Zinn, 1990) with features of Cognitive Therapy (Beck, Rush, Shaw, \& Emergy, 1979; Teasdale, Segal, \& Williams, 1995). The original idea behind $\mathrm{MBCT}$ was to train participants to disengage from automatic negative thinking patterns that arise during dysphoric mood and facilitate relapse. However, there is reason to believe that MBCT might also increase participants' capacity for the experience of positive affect. We therefore investigated whether participation in could teach vulnerable individuals to make better use of natural rewards in daily life and to generate higher levels of positive emotions.

In Chapter 8, we examined whether the current practice to exclude patients with only one or two prior episodes of major depression from most MBCT trials is justifiable. Exclusion of this patient group is based on two earlier studies which had found that only patients with three or more prior depressive episodes had a reduced risk of relapse after MBCT (Ma \& Teasdale, 2004; Teasdale, et al., 2000). However, if MBCT indeed facilitates an affective processing style associated with increased resilience, then $\mathrm{MBCT}$ should be beneficial for everyone at risk for depression (independent of the number of prior depressive episodes). Wrongly excluding people with less than three prior episodes of major depression from MBCT trials would deny them the chance to become more emotionally resilient.

Chapter 9 provides a general discussion of the studies included in this thesis, along with clinical and societal implications and suggestions for future research. 


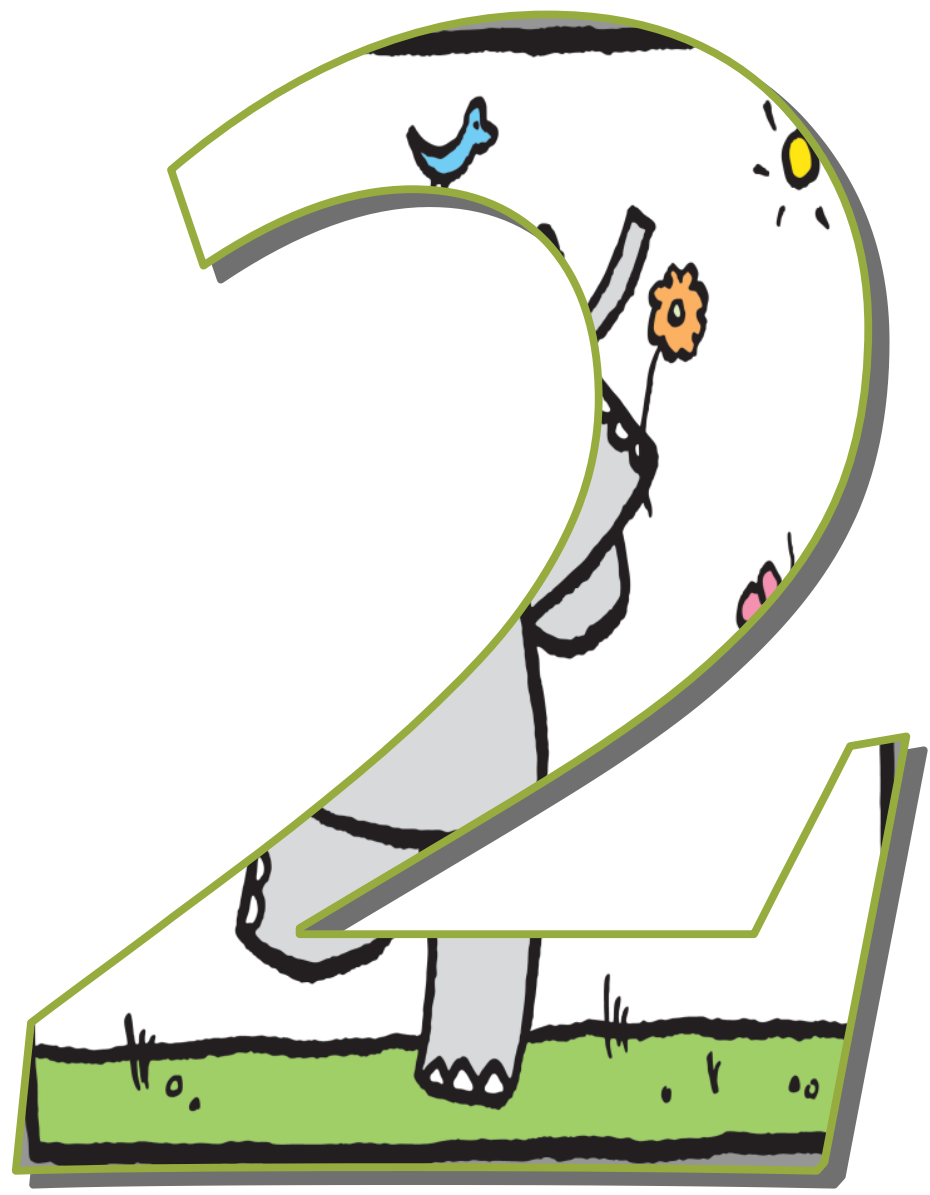

The role of affective processing in vulnerability to and resilience against depression 
Published as:

Geschwind, N., van Os, J., Peeters, F., \& Wichers, M. (2009). The role of affective processing in vulnerability to and resilience against depression. In C. M. Pariante, R. M. Nesse, D. Nutt \& L. Wolpert (Eds.), Understanding depression: A translational approach (pp. 181-192). NY: New York: Oxford University Press Inc.

\section{INTRODUCTION: DEPRESSION AS A COMPLEX DISORDER}

Unlike Mendelian diseases, depression is not caused by a single gene alteration but is the product of multiple interacting causes (Cannon \& Keller, 2005; Van Praag, De Kloet, \& Van Os, 2004b). Recent findings underscore just how complex these interactions can be. Genes may interact with the environment (e.g. the serotonin transporter polymorphism has been found to interact with stress) (Caspi, et al., 2003) or increase the risk of exposure to certain environments (e.g. through mediation of such personality traits as neuroticism or sensation-seeking) (Van Praag, et al., 2004b). Environmental risk factors may interact or correlate with each other (e.g. childhood adversity was found to be associated with a higher occurrence of life events) (Wichers, et al., 2009c). Gene $x$ gene $x$ environment interactions have also been reported. For example, regarding risk for depression, the serotonin transporter polymorphism and a gene coding for brain-derived neurotrophic factor (BDNF) were shown to interact with each other as well as with childhood maltreatment and social support (Kaufman, et al., 2006). Furthermore, new insights demonstrate that genetic regulation is more complex than previously thought (Pearson, 2006). Genes can be functionally altered by insertions, deletions or duplications. Furthermore, as has been demonstrated in the rapidly developing field of epigenetics, a gene can be silenced through the addition of a methyl group to the gene. Methylation of genes is reversible and can be influenced by environmental factors as well as be inherited (Mill \& Petronis, 2007; Pearson, 2006). In a study on rat pups, for example, it was found that the degree of maternal care influenced gene methylation, which in turn was associated with altered HPA axis functioning (Fish, et al., 2004).

Given this challengingly complex situation, there is an urgent need for multidisciplinary studies involving epidemiology, psychology, genetics, psychiatry, pharmacology and neuroscience (Moffitt, Caspi, \& Rutter, 2005; The European Network of Schizophrenia Networks for the Study of Gene-Environment Interactions (EU-GEI), 2008). Where possible, such studies should focus on endophenotypes rather than on disorder outcomes (Caspi \& Moffitt, 2006). Endophenotypes are intermediate phenotypes (expressions of genes) that lie 
somewhere on the pathway between disease-promoting genetic variation, biological vulnerability and disorder (Cannon \& Keller, 2005). They are assumed to be more biologically homogeneous than the current nosological categories of disorders. Examining endophenotypes may facilitate the identification of susceptibility genes and gene-environment interactions (Bearden \& Freimer, 2006). The nature of endophenotypes may be biological (e.g. HPA axis functioning) or psychological (e.g. sensitivity to stress).

Several assessment issues are important especially when studying psychological endophenotypes. Assessment should be (i) repeated (rather than cross-sectional / one-time assessment), because repeated measurements increase reliability and sensitivity; (ii) prospective (rather than retrospective); and (iii) based on a short and recent time span (rather than covering longer periods of time), thereby decreasing memory bias (Delespaul, 1995). In contrast to clinical interviews and standardized questionnaires, the experience sampling method (ESM) meets all these requirements (see Figure 1). In ESM research, participants' reactions to random real-life situations are recorded in a booklet at multiple times a day for several days, assessing context, emotions and cognitions as they occur. This repeated measurement of daily-life situations endows ESM with particularly high ecological validity and reliability (De Vries, 1992; Eck, Nicolson, \& Berkhof, 1998). ESM thus makes it possible to study disorder-relevant endophenotypes in daily life, in that is, in the context in which they take place. Examples of ESM endophenotypes are stress sensitivity (often defined as negative affect reactivity to daily-life stressors) or reward experience (often defined as positive affect reactivity to pleasant daily-life events) (e.g., Wichers, et al., 2009a) . The measurement of ESM endophenotypes can of course be combined with genetic information or biological measurements (e.g. cortisol, see Wichers, et al., 2008c). The above-mentioned features make ESM an ideal instrument for the study of gene-environment interaction research. 


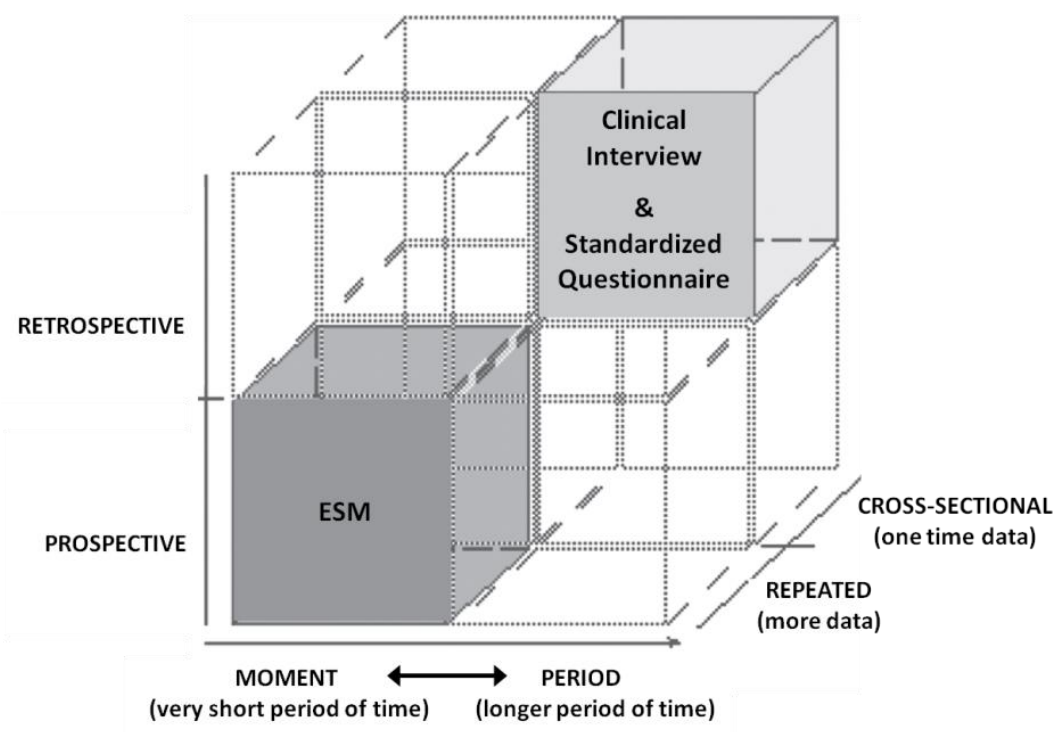

Figure 1. Comparison of the experience sampling method (ESM) and conventional measures. ESM is prospective, repeated and concerned with very short moments of time, compared to conventional methods such as clinical interviews and standardized questionnaires. Figure reproduced with permission from Delespaul, 1995.

In the past, research has largely focused on what makes us vulnerable to psychopathology (Seligman \& Csikszentmihalyi, 2000). With the advent of the positive psychology movement, researchers have started to show more interest in protective influences (Gable \& Haidt, 2005). While some people are particularly vulnerable to becoming depressed, others show remarkable resilience. Studying both vulnerability and resilience will enable us to get a more complete picture of how to best prevent and treat depression. This chapter therefore deals with research into both vulnerability to and resilience against depression with the complexity of co-occurring and interacting genetic and environmental processes in mind. The discussion focuses on the latest findings concerning the role of affective processing in relation to vulnerability and resilience, with special attention to gene-environment research using ESM.

\section{VULNERABILITY TO AND RESILIENCE AGAINST DEPRESSION}

Depression is a multifaceted disorder: emotion, perception, cognition, selfesteem, personality, social support and coping are only some of the many factors involved in its aetiology and maintenance. However, the scope of this chapter is limited to affective processing. Investigations into the factor structure of emotion 
have converged on two-factor models, commonly labelled positive and negative affect (PA and NA, respectively) (Almagor \& Ben-Porath, 1989; Watson \& Tellegen, 1985). PA is associated with behavioral approach and characterized by such feelings as enthusiasm, interest and happiness. NA is associated with behavioral withdrawal and includes such feelings as distress, sadness and hostility. Evidence suggests that PA and NA are related yet independent systems (Watson, et al., 1999).

In depression, the processing of both NA and PA appears to be altered (Leppänen, 2006). Increased NA as well as a reduced capacity to experience PA (anhedonia) are core symptoms of depression (American Psychiatric Association, 2000). Much if not most of people's emotional experience is influenced by events in their daily lives. A genuine smile can make someone feel happy and elated, whereas a careless word can make someone feel upset or angry. Tendencies to react to daily-life events in a certain way not only have short-term effects (in terms of e.g. altered mood) but also appear to have long-term consequences. For example, the tendency to react to daily-life stressors with a large increase in NA appears to be a risk indicator for depression (Jacobs, et al., 2006; Wichers, et al., 2007b). On the other hand, a tendency to experience more positive emotions has been found to have several protective effects (e.g. Danner, et al., 2001; Fredrickson, 2001).

\section{Vulnerability to depression: negative affect and stress sensitivity}

Increased NA and withdrawn behavior are core symptoms of depression. As argued above, stress sensitivity is likely a main contributor to daily-life NA. Stress sensitivity also has biological plausibility as an endophenotype, given that depressed individuals frequently display excessive HPA axis activation and that HPA axis functioning has been linked to stress responses (e.g. see Hasler, Drevets, Manji, \& Charney, 2004). An ESM twin study showed that participants with a cotwin with lifetime depression have a different diurnal cortisol profile than those without, suggesting that altered HPA axis functioning is an indicator of depression liability, and not just a consequence of being depressed (Wichers, et al., 2008c).

It has been shown that both genetic and environmental risk factors can produce long-term increases in sensitivity to stressful events. For example, women with a history of childhood abuse displayed an augmented ACTH and cortisol response as well as elevated heart rate in a laboratory public-speaking psychosocial stress task of public speaking (Heim, et al., 2000). A history of childhood abuse has also been found to make women more sensitive to the depressogenic effects of stressful life events (SLEs) (Kendler, Kuhn, \& Prescott, 2004). In a study by van Os and Jones (1999), higher maternal neuroticism was 
associated with higher sensitivity to SLEs, indicating familial transmission of vulnerability factors. Momentary assessment technology using ESM is particularly well suited to provide ecological validation of the notion of sensitivity to stress as embodied in, for example, the personality trait neuroticism. Recent ESM data indicate that neuroticism expresses itself in daily life through more NA, longerlasting NA and stronger NA reactivity to stressful daily events (Jacobs et al. submitted). Poorer childhood mental health and higher levels of childhood neuroticism were associated with higher sensitivity to SLEs, too, suggesting that altered stress sensitivity may be one of the mechanisms linking poorer childhood mental health to enduring affective psychopathology in adulthood (Van Os \& Jones, 1999). Taken together, these studies suggest that heightened stress sensitivity is a risk factor and endophenotype for depression.

A recent ESM study confirmed that daily-life stress sensitivity (operationalized as NA reactivity to daily-life stressors) is an important endophenotype for depression. Study participants with the highest genetic risk of depression (measured by co-twin lifetime depression status) reacted to daily-life stressors with more NA than did participants with low genetic risk (Wichers, et al., 2007b). Prospective follow-up measurements showed that daily-life stress sensitivity indeed predicts future depression (Wichers, et al., 2007b). As a whole, these studies show that depression is associated with higher stress sensitivity and suggest that heightened stress sensitivity represents a vulnerability to depression rather than a consequence of being depressed.

\section{Sensitization and stress sensitivity}

Knowing that stress sensitivity plays an important role in depression, it is important to examine the mechanisms through which people become more sensitive to stress. A likely mechanism contributing to the development of stress sensitivity is 'sensitization'.

In order to explain the long-term effects of early stress on later adult vulnerability, Post (1992) suggested (on the basis of animal laboratory studies) that, in some individuals, a process of 'sensitization' to stressors occurs over the course of development. Early stress may trigger structural changes due to the induction of gene-transcription factors. These structural changes likely result in long-term changes in the expression of neurotransmitters, receptors and neuropeptides. These changes are assumed to induce progressively lower thresholds to stress, so that with each further exposure less stress is required for similar behavioral (and biological) responses (Monroe \& Harkness, 2005; Post, 1992). Findings concerning stress and depression in the literature - for example the fact that recurrent episodes of depression become more and more 
independent of major life stressors and can eventually be triggered even by minor events - support the notion that a process of sensitization may be involved (Kendler, Thornton, \& Gardner, 2001; Monroe \& Harkness, 2005).

A recent ESM study that tested the hypothesis that sensitization processes underlie the development of stress sensitivity, found that previously experienced stress exposures - such as childhood adversity and SLEs in adulthood - increased adult stress sensitivity to small stressors in the flow of daily life in a doseresponse relationship: the greater the number of adversities people had faced, the higher their stress sensitivity. Furthermore, genes appeared to potentiate this effect: participants with a high genetic risk for depression developed higher levels of daily-life stress sensitivity in response to pre-natal and post-natal adversities than did those with a low genetic risk (see Figure 2). Childhood adversity seemed to play the most important role in the vicious circle of increased stress sensitivity in those at high genetic risk for depression, because it was associated with both increased stress sensitivity and a higher occurrence of SLEs. In fact, the effect of adult SLEs on stress sensitivity disappeared when controlled for childhood adversity (Wichers, et al., 2009c) . An effect of childhood adversity on stress sensitivity was also found in another ESM study on frequent attendees of general practitioners (Glaser, van Os, Portegijs, \& Myin-Germeys, 2006), as well as in laboratory and naturalistic studies (e.g. Heim, et al., 2000; Kendler, et al., 2004).

Taken together, these studies show that adverse events render especially the genetically vulnerable more sensitive to stress, and support the hypothesis that genes contribute to vulnerability partly by facilitating the process of sensitization after stress exposure. However, many questions remain. For example, it is unknown what the exact mechanisms of stress sensitization are or how epigenetic changes influence stress sensitization. 


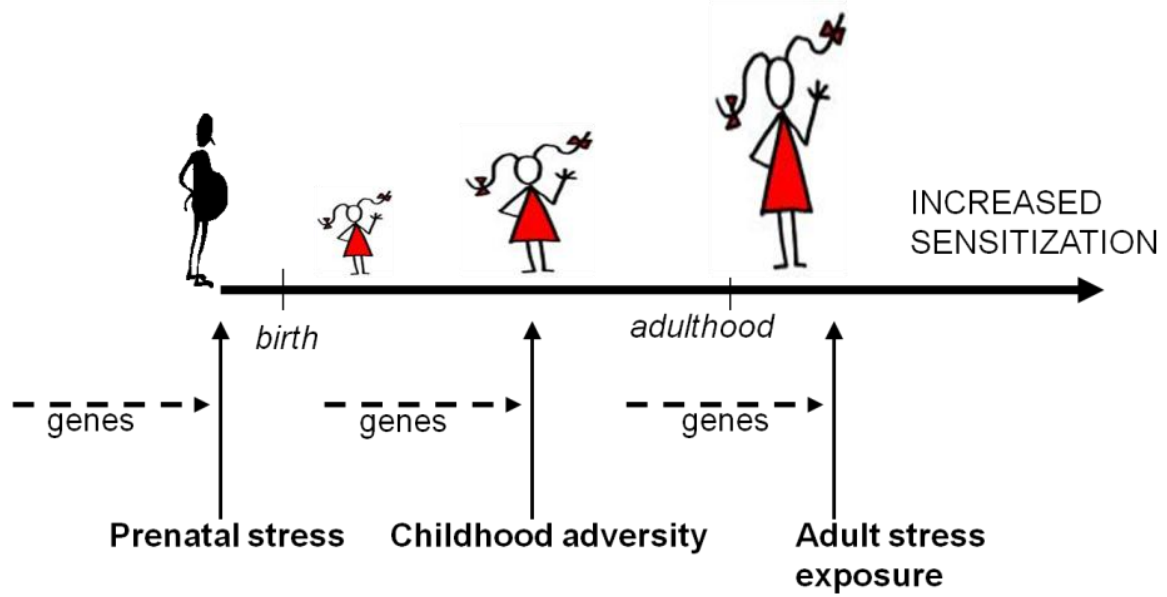

Figure 2. Stress exposures during the course of development are hypothesized to increase future stress sensitization. Genetic factors may impact on sensitization by increasing the effects of stressors on the sensitization process. Thus, genes likely interact with environmental stressors at different stages of development.

\section{Resilience against depression: the role of positive emotions}

Along with the frequent experience of negative emotions, the diminished experience of positive emotions lies at the heart of depressive symptomatology. Anhedonia is a core symptom of major depressive disorder, and experimental studies showed that depressed individuals generated less positive affect from pleasant stimuli than did non-depressed individuals (Dunn, Dalgleish, Lawrence, Cusack, \& Ogilvie, 2004; Sloan, Strauss, Quirk, \& Sajatovic, 1997). However, compared to the extensive literature on negative emotions, relatively little attention has been paid to the role of positive emotions in depression. Some studies, though, have been performed in this area.

Positive emotions and having a positive attitude seem to have beneficial effects on mental and physical health (Seligman, Steen, \& Peterson, 2005). For example, positive emotions speed up cardiovascular recovery from stress and improve immune system functioning, and are associated with increased longevity (Fredrickson, 2001; Tugade \& Fredrickson, 2004; Tugade, Fredrickson, \& Barrett, 2004). In Danner and colleagues' well-known nun study (2001), positive emotional content in early-life autobiographies was strongly associated with longevity 60 years later, with a 2.5 fold difference in risk for mortality between nuns in the lowest and those in the highest quartile of positive emotion expression. A metaanalysis of cross-sectional, longitudinal and experimental studies demonstrated that happiness was associated with and preceded success, indicating that 
happiness facilitates accomplishment (Lyubomirsky, King, \& Diener, 2005). In a study in which women with chronic pain were interviewed weekly, higher levels of overall PA predicted lower levels of pain in subsequent weeks, demonstrating that positive affect can be a source of resilience in chronic pain. Higher weekly PA also resulted in lower negative affect both directly and in interaction with stress and pain (Zautra, Johnson, \& Davis, 2005).

The latest ESM studies that additionally examined gene-environment interactions confirm the protective function of positive emotions. An ESM twin study showed that the experience of positive emotions during moments of dailylife stress protects against stress sensitivity by buffering negative reactivity to stressful events (Wichers, et al., 2007a). Moreover, the experience of positive emotions also attenuated the expression of genetic vulnerability on negative mood bias in the flow of daily life (Wichers, et al., 2007a). On average, having a twin (especially a monozygotic twin) with a lifetime history of depression is associated with increased NA in response to stressful situations (Wichers, et al., 2007b). However, this effect of genetic vulnerability is weakened during the coexperience of positive emotions (Wichers, et al., 2007a).

The protective effect of positive emotions on genetic vulnerability was also found in ESM studies that examined specific genes, for example the gene encoding for brain-derived neurotrophic factor (BDNF). BDNF is important for neuronal survival and synaptic plasticity (Nair \& Vaidya, 2006). Stress has been found to decrease BDNF expression in the hippocampus (Duman \& Monteggia, 2006), and there is evidence that BDNF plays a role in such stress-related disorders as depression (Chen, et al., 2006; Hashimoto, 2007; Hashimoto, Shimizu, \& lyo, 2004; Lang, et al., 2005; Nair \& Vaidya, 2006). The gene coding for BDNF contains a functional polymorphism ( $\mathrm{Val}{ }^{66} \mathrm{Met}$ ). Of the two genetic versions, Valine (Val) is the more active variant and is therefore associated with higher BDNF secretion than is Methionine (Met). A recent ESM study found that carriers of the BDNF Val/Met combination displayed a significantly larger NA response to social stress than did carriers of the Val/Val combination (Wichers, et al., 2008b). Interestingly, this gene-environment interaction was moderated by the experience of positive emotions. The experience of positive emotions during social stress decreased BDNF genetic moderation of the NA response. When $\mathrm{Val} /$ Met carriers experienced high levels of positive emotion at times of social stress, their NA levels were comparable to those of BDNF Val/Val carriers (see Figure 3).

The studies described above suggest that genetic effects do not influence stress sensitivity in a deterministic manner and may be modified by targeting the 
ability to generate positive emotions in daily life. Furthermore, a decreased ability to generate positive emotions may contribute to a loss of resilience against stressful events in daily life. Therefore, since daily-life stress sensitivity is likely on the causal pathway to depression, an increased ability to generate positive emotions may contribute to resilience against the development of depression. This hypothesis is confirmed by a randomized controlled trial that compared the antidepressant imipramine with placebo. A six-week treatment with imipramine decreased daily-life stress sensitivity (measured with ESM) and increased the amount of positive emotion generated from daily-life activities (Wichers, et al., 2009a). Importantly, only increases in the ability to experience positive emotion discriminated between treatment responders and non-responders. This implies that the restoration of the ability to experience positive affect is required for response to treatment in depression, and further underlines the value of targeting positive emotions in particular.

To conclude, the existing literature on positive emotions demonstrates that positive emotions have important protective functions and that mechanisms of resilience related to the experience of positive emotions clearly deserve further attention in future research and therapy efforts. The potential of positive emotions to diminish the expression of genetic vulnerability suggests that focusing on positive emotions may be especially beneficial in certain subgroups. This possibility is discussed in the next paragraph.

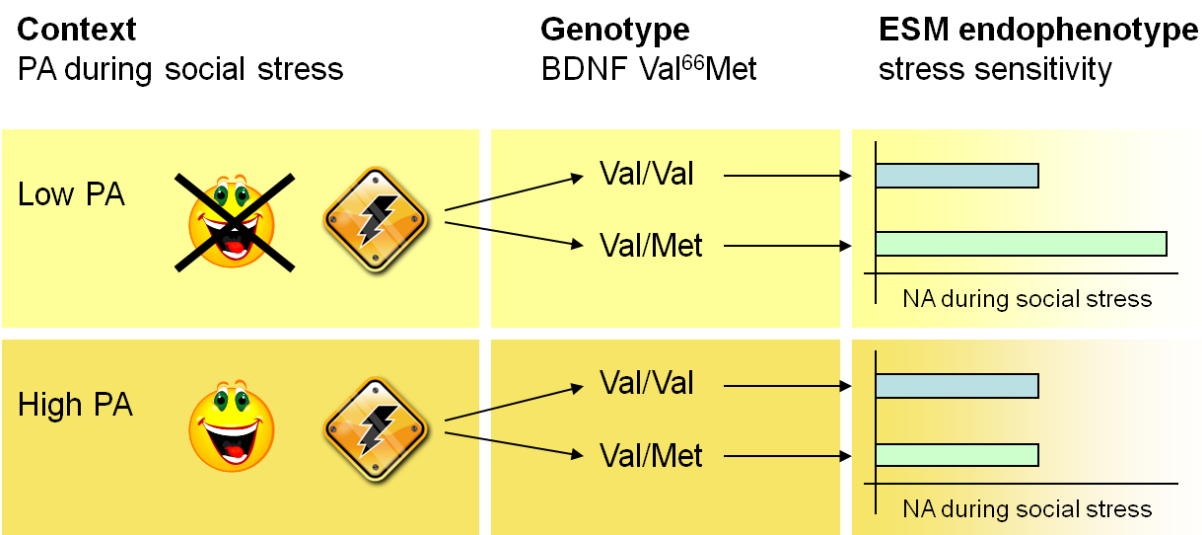

Figure 3. The experience of positive emotions at times of social stress decreases the genetic moderation of the BDNF Val ${ }^{66}$ Met polymorphism on the NA response to social stress. When PA is low, Val/Met carriers experience significantly more NA than do Val/Val carriers during social stress. When PA is high, however, this difference in sensitivity to social stress diminishes (graphic summary of data from Wichers et al. 2008b). 
Targeting positive emotions: personalized medicine?

The above-mentioned finding that BDNF Val/Met carriers in particular benefit from experiencing positive emotion at times of social stress (Wichers, et al., 2008b) suggests possible advantages of 'personalized medicine' (targeting treatment to an individual's needs on the basis of personal characteristics). Targeting the ability to experience positive emotions appears to be especially relevant in individuals who have at least one copy of the Met variant, because teaching them to experience more positive emotions may have a protective influence on their genetic predisposition to experience more social stress.

Other studies suggest that variations in the catechol-O-methyl transferase (COMT) Val ${ }^{158}$ Met polymorphism may be associated with the ability to experience positive emotions. COMT is an enzyme that breaks down dopamine, and dopamine has been found to be associated with positive affect (Burgdorf \& Panksepp, 2006). Met is less active than Val, leading to a lower levels of COMT and, as a result, higher prefrontal levels of dopamine (Egan, Goldman, \& Weinberger, 2002). In a recent ESM study on the COMT Val ${ }^{158}$ Met polymorphism, it was found that the ability to generate positive emotions from daily-life events increased with an increase in the number of the subject's Met alleles (Wichers, et al., 2008a) (see Figure 4). This raises the question whether targeting the ability to experience positive emotions may be especially useful in Val/Val carriers because they seem to be less efficient in experiencing PA - or in Met/Met carriers, because they may have a greater capacity for experiencing PA. Two recent studies show that having at least one Met allele is associated with a faster (Yoshida, et al., 2008) and a better (Baune, et al., 2008) response to antidepressant medication (although see also Szegedi, et al., 2005). This may suggest that Met/Met carriers have a higher natural capacity to recover from depression, possibly via PA, and that especially carriers of $\mathrm{Val} / \mathrm{Val}$ may benefit from additional treatment that targets positive emotions. However, such suggestions are highly speculative at this point.

To conclude, while the notion of 'personalized medicine' is certainly attractive, much more research is needed in order to determine what the optimal individual treatment strategies may be. 


\section{Context}

Pleasant daily-life events
Genotype

COMT Val ${ }^{158} \mathrm{Met}$
ESM endophenotype

reward experience
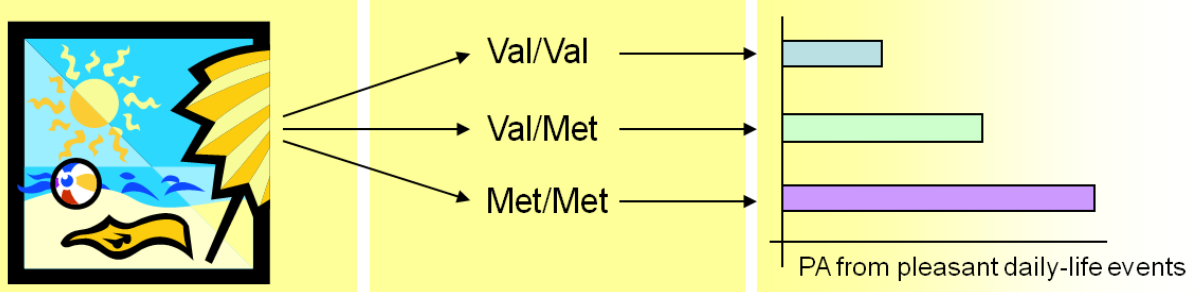

Figure 4. Genetic moderation by the COMT Val ${ }^{158}$ Met polymorphism on the PA response to pleasant daily-life events (here called 'reward experience'). In a recent ESM study, the ability to generate positive emotions from daily-life events was found to increase with the number of Met alleles of the subject (graphic summary of data from Wichers et al. 2008a).

Targeting positive emotions: how?

Increasing the ability to experience positive emotions could play an important role in making people less stress sensitive and more resilient against depression. Furthermore, response to treatment of depression may be conditional on restoration of the ability to experience PA (Wichers, et al., 2009a). The question arises how PA may be best targeted, either as prevention strategy to make people more resilient against depression, or in combination with pharmacological treatment in order to obtain an optimal treatment response.

Meditation-based techniques, such as mindfulness-based cognitive therapy (MBCT), may offer a way to modify the experience of PA. More advanced meditators have been found to possess greater self-awareness and to experience more positive emotions (Easterlin \& Cardena, 1998), and people have been found to report more positive emotions when in a mindful compared to a non-mindful state (Brown \& Ryan, 2003). During mindfulness training sessions, people are trained towards increased moment-to-moment awareness of experience, resulting in increased openness or receptiveness (Baer 2003). Although studies have shown that mindfulness training is effective in reducing depressive symptoms and relapse probability (Baer, 2003; Coelho, Canter, \& Ernst, 2007; Teasdale, et al., 2000), the mechanism remains unknown. It can be hypothesized that mindfulness training is a tool that increases the ability to make use of natural, moment-to-moment rewards in the environment, thereby increasing positive affect. Whether this is indeed the case should be evaluated in future research. 


\section{CONCLUSION / FUTURE DIRECTIONS}

Sensitive and ecologically valid gene-environment interaction research focusing on daily-life (ESM) endophenotypes is badly needed in order to further unravel the very complex gene-environment interactions associated with depression. Recent ESM studies have shown that heightened stress sensitivity is both a risk factor and an endophenotype of depression, and suggest that PA plays a role in making people more resilient to stress. Focusing treatment and prevention efforts on heightening positive affect therefore appears to be a promising venue to reduce stress sensitivity. Further research is necessary in order to answer such questions as how the ability to experience positive emotions is best modified, and who will benefit most from a treatment aimed at increasing PA.

\section{Acknowledgement}

Marieke Wichers was supported by the Dutch Medical Council (VENI grant nr 916.76.147). 



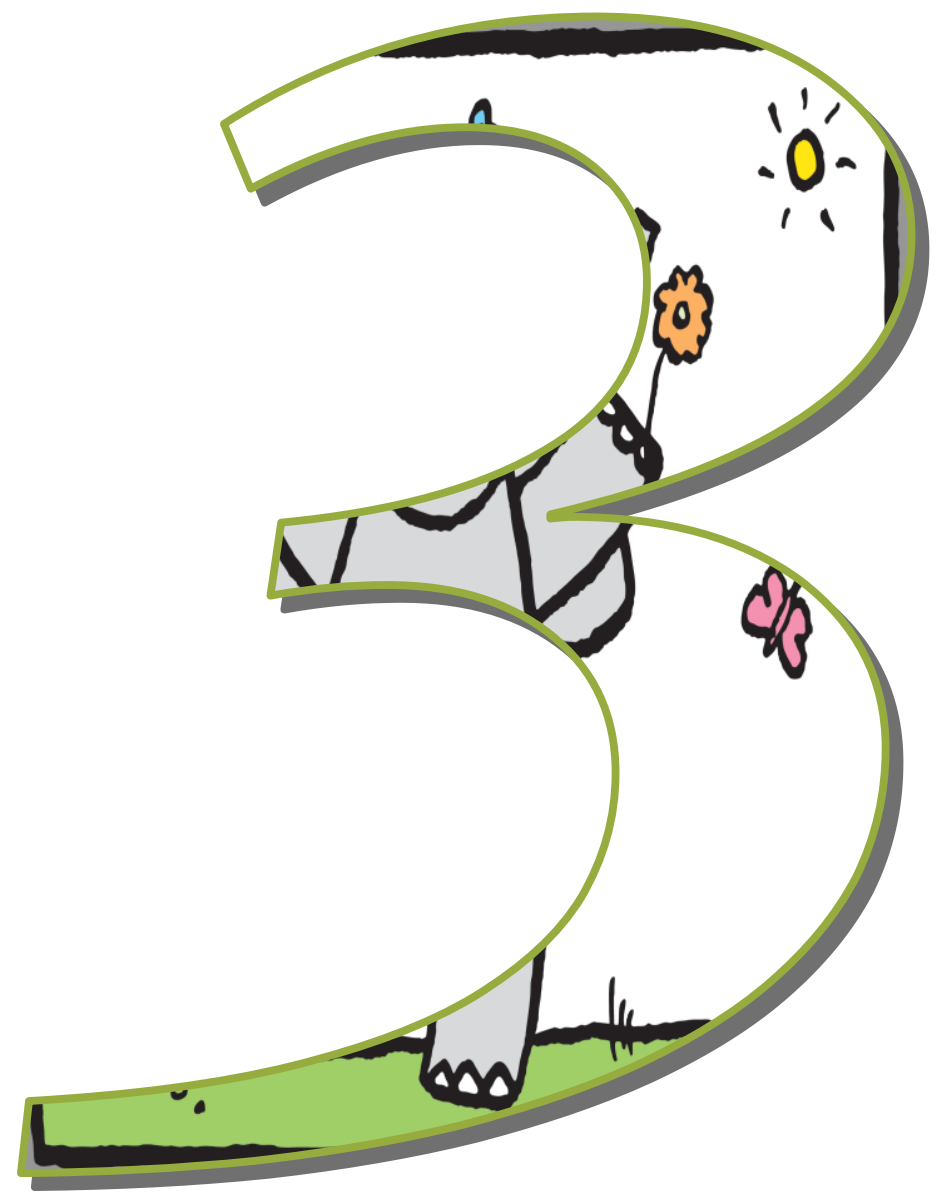

Scars in depression: is a conceptual shift necessary to solve the puzzle? 


\begin{abstract}
Although clinical findings suggest that in the aftermath of depression a process of 'scarring' may ensue, research examining the issue of 'scars' (including biological, psychological and cognitive changes) has remained largely inconclusive. This paper proposes a new approach to the concept of 'scar', that is (i) based on a dimensional view on depression, (ii) uses methods that take into account the dynamic interplay between the person and his context, (iii) differentiates between scars following depression and scars following the factor that actually caused the depression such as stress and (iv) introduces a dynamic view of the concept of 'scar' in that it hypothesizes that scars can wax and wane. This approach may stimulate the discovery of new entries in the puzzle underlying the ontogenesis of vulnerability and resilience. Furthermore, it may provide insights that help to develop new therapies for depression.
\end{abstract}

Key words: Depressive disorder, dimensional approach, experimental designs, treatment.

\title{
Published as:
}

Wichers, M. C., Geschwind, N., van Os, J., \& Peeters, F. (2010). Scars in depression: Is a conceptual shift necessary to solve the puzzle? Psychological Medicine, 40(3), 359-365. doi: 10.1017/s0033291709990420 
Major depressive disorder (MDD) is, at least in secondary and more specialized care settings with morbidity concentration, a chronic psychiatric disorder with residual symptoms, frequent relapses, and estimates of recurrence ranging from $33 \%$ to $70 \%$ within several years (Pettit, Lewinsohn, \& Joiner, 2006).

Previous research has shown that the causal role of severe life-events is smaller in recurrences than in first-onset episodes of MDD (Monroe \& Harkness, 2005; Stroud, Davila, \& Moyer, 2008). This observation was described by Post (Post, 1992) as a process of behavioral 'sensitization' and electrophysiological 'kindling', suggesting that a depressive episode leaves some traces that persist after remission and recovery, and render individuals vulnerable to the onset of new episodes under the influence of only moderate or, eventually, no psychosocial stress. This idea is known as the scar hypothesis of depression (Lewinsohn, Steinmetz, Larson, \& Franklin, 1981). Scar theories assume that something, presumably encoded at the biological level, changes during an episode of depression, inducing a long-lasting change and increasing the likelihood of future episodes (Burcusa \& lacono, 2007). Scar research, however, is not confined to the neurobiological domain. In contrary, studies examining scars in depression have focussed on potential scars within a broad variety of domains (psychosocial, cognitive, emotional and others) (Beevers, Rohde, Stice, \& Nolen-Hoeksema, 2007; Burcusa \& lacono, 2007; Ormel, Oldehinkel, \& Vollebergh, 2004b; Shea, et al., 1996). In this manuscript, therefore, the term scar may refer to all possible changes in cognition, emotion, behavior or biology that develop in the aftermath of a depressive mood state and result in a stable increase in vulnerability.

Studies that address the scar hypothesis ideally assess putative factors that may become scarred before and after an episode of MDD in participants without residual symptoms. In studies that attempted to apply such a rigorous design, several potential scars were examined including depression-related cognitions (Barnett \& Gotlib, 1988; Lewinsohn, et al., 1981), personality (De Fruyt, Van Leeuwen, Bagby, Rolland, \& Rouillon, 2006; Duggan, Sham, Lee, \& Murray, 1991; Rohde, Lewinsohn, \& Seeley, 1990; Shea, et al., 1996); (Ormel, et al., 2004b), psychosocial disability (Ormel, Oldehinkel, Nolen, \& Vollebergh, 2004a; Rohde, et al., 1990), social skills (Zeiss \& Lewinsohn, 1988) as well as rumination, selfesteem and negative emotionality (Beevers, et al., 2007). However, in all these studies virtually no evidence was found for the scar hypothesis. Any changes in the aforementioned variables were either concomitants of current (residual) depressive symptoms or scores were already elevated before the onset of the episode of MDD thus likely representing premorbid risk factors rather than scars. The field is thus left with a situation in which vulnerability for onset of episodes of 
MDD seems to increase as a function of the number of episodes, suggestive of scarring, yet studies that try to examine the mechanisms involved fail to shed any light on the issue.

We will argue that methodological and conceptual complexities may underlie this discrepancy and propose a new approach to the concept of 'scar' for future research.

\section{WHERE AND HOW SHOULD WE LOOK FOR THE SCAR?}

\section{Dimensional approach}

Although the categorical approach of MDD in the DSM-IV may be useful in clinical settings, depression is probably best conceptualized as a dimensional construct rather than as a discrete diagnostic entity (Hankin, Fraley, Lahey, \& Waldman, 2005; Kendler \& Gardner, 1998; Slade, 2007), although some controversy exists in the literature (Beach \& Amir, 2003; Coyne, 1994; Flett, Vredenburg, \& Krames, 1997; Solomon, Ruscio, Seeley, \& Lewinsohn, 2006). Strong arguments for the dimensional view of depression include the fairly consistent finding that subthreshold depression strongly increases risk for future MDD (Cuijpers \& Smit, 2004) and that subthreshold depression impacts considerably on functioning (Judd, et al., 2002). The influence of underlying etiological factors is therefore unlikely to be limited to man-made DSM-IV boundaries. Likewise, it is improbable that scars would only develop after an episode that fulfils the DSM-IV criteria. A more plausible hypothesis is that scars, if any, would develop proportionally to the amount of depressive symptoms experienced. This would mean that scars already develop gradually as a result of subtle sub-clinical depressive symptoms before the onset of a full-blown episode of MDD. This gradually increasing scar, as the catalyser of a vicious circle, may thus additionally constitute a risk factor for the first episode of MDD (see Figure 1). Likewise, 'scarring' residual symptoms after MDD may additionally constitute a risk factor for relapse. This principle can be compared to the proposed vicious circle of the global warming of the earth. The warming of the atmosphere makes the ice on the North pole melt. This allows the sun to shine upon the surface of the sea that was previously covered with ice, enabling it to warm up the sea even further and cause melting of additional ice. In this example, the melting of the ice would be the gradually developing 'scar' that subsequently by itself further increases the speed of warming up, eventually causing a point of global warming where humans experience the consequences. Likewise, in the case of MDD, negative emotionality and low self-esteem can be perceived as potential risk catalysers: they may be caused by negative 
environmental events, and at the same time induce risk for future negative (interpretations of) situations that, in turn, will build up even more scars. Similarly, exposure to stress (Hammen, 1991) itself could be a potential risk catalyser: depressive symptoms may foster life circumstances that increase the likelihood of exposure to stress that in turn further increase the individual's vulnerability to depression. If the proposition regarding the 'gradually increasing scar' is true then it is impossible to identify these scars using the dichotomous approach as described in the introduction, since pre-morbid alterations will be identified as risk factors rather than scars resulting from sub-clinical depressive symptoms.

Thus, we propose that the scars that researchers wish to identify may develop gradually along the life-cycle, proportionally to the severity and duration of the depressive symptoms experienced.

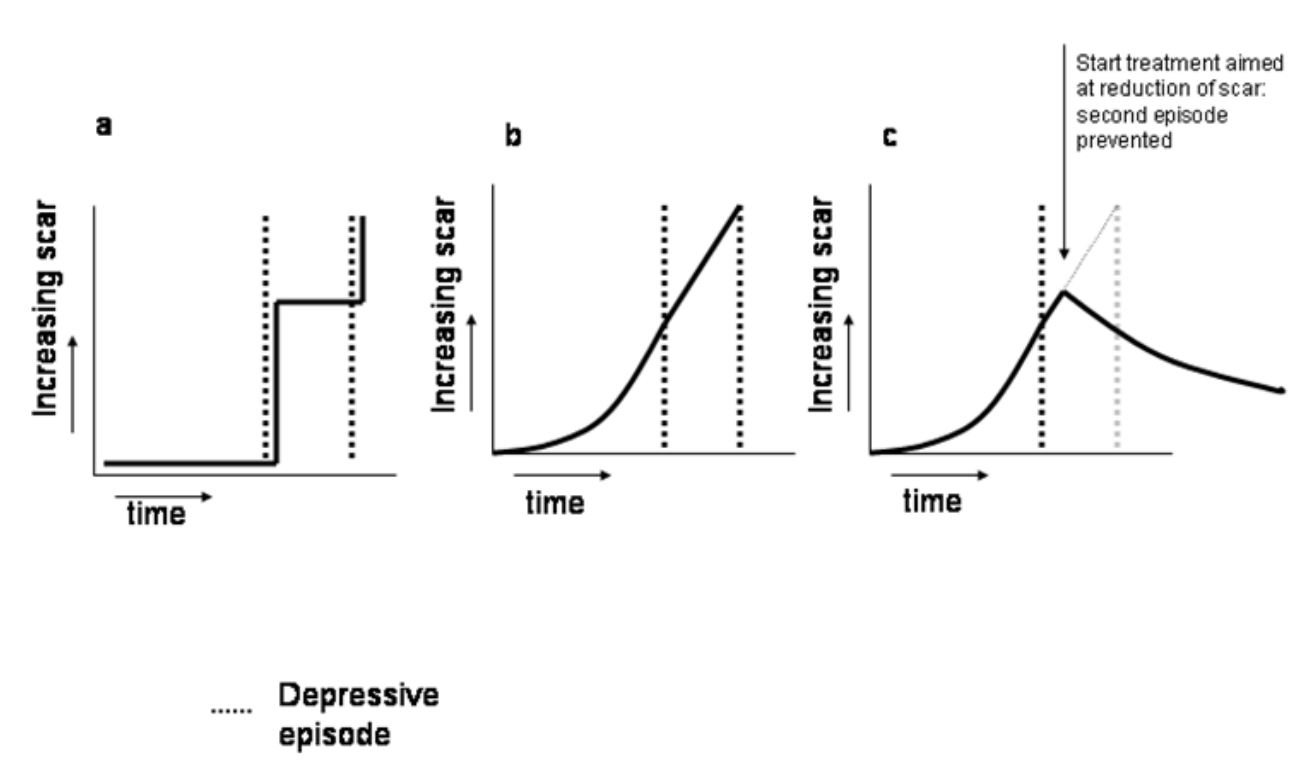

Figure 1. 1a represents the dichotomous scar model while $1 \mathrm{~b}$ and $1 \mathrm{c}$ represent the continuous scar model. The figures are valid in a context where the individual experiences stress or sub-clinical depressive symptomatology. The $x$-axis represents time and the $y$-axis the increasing scar. The dotted lines represent episodes of depression. In Figure 1a, the scar develops suddenly following an episode of depression. In Figure $1 \mathrm{~b}$, the scar develops gradually over time regardless of the arbitrary boundaries of fulfilling criteria for depression. Figure 1c depicts the hypothesis that scars are reversible and that (new) treatments aimed at reducing scars may prevent the development of further episodes of depression due to their effect on scar development. 


\section{Sensitive to person-context interactions}

Cross-sectional, non-dynamic methodology in depression research will likely obscure the effects of scarring. Without taking into account the dynamic interplay between the person and his/her context, measures may not be sensitive enough to make a scar effect visible. For example, Segal and colleagues found that a greater cognitive reactivity of remitted depressed patients following induction of depressed mood increased the risk for relapse (Segal, et al., 2006). Thus, the (re)activation of depressive thinking styles triggered by temporary dysphoric states may indicate vulnerability. In this study, dysfunctional cognitive attitudes would likely have remained inactive in the absence of an experimental mood induction paradigm, so that differences between controls and remitted patients would not have been detected. Thus, it is possible that scars are not immediately accessible and measurable with questionnaires, but that they can only be made visible using challenging experimental designs that activate a participant's vulnerability. Tools that are able to reveal subtle dysfunctions following experimental induction are available in the psychiatric literature. However, up to now they are not commonly used to evaluate possible scar effects. Examples of experimental tools in the biological domain are challenges of serotonergic pathways in the brain and the hypothalamic-pituitary-adrenal (HPA) axis. The former is achieved by acute tryptophan depletion (ATD) through lowering levels of tryptophan (essential for 5-HT synthesis) that cannot be synthesized by the body. Bhagwagar and Cowen (Bhagwagar \& Cowen, 2008) recently suggested that ATD reveals latent dysfunctions in brain circuits that are associated with regulation of emotion in MDD vulnerable subjects. Another biological experimental tool that may possibly reveal scars is the Dexamethasone Corticotropin Releasing Hormone suppression (DEX/CRH) test. No HPA-axis abnormalities (e.g., alterations in basal cortisol secretion) are reported in recovered patients unless challenged by for example the DEX/CRH-test (Zobel, et al., 2001). To our knowledge, no studies have used these methodologies to investigate the process of scarring.

Another type of design that incorporates the dynamic interplay between an individual and his/her environment is the Experience Sampling Method (ESM) (Csikszentmihalyi \& Larson, 1987; Delespaul, 1995). In ESM research, individuals are asked to fill in a diary in response to a beep signal at random unpredictable moments of the day. The diary provides information on their current mood state, physical state, current activities, appraisal of the current social situation and recent (past hour) events etc. This method enables investigators to assess subtle changes in dynamic real-life person-context interactions instead of traits measured in an experimental laboratory setting which typically isolates subjects 
from their daily-life context. For example, the negative affective response to minor daily-life stressors - daily-life stress sensitivity - has been revealed as an important risk factor for the development of future depressive symptomatology (Wichers, et al., 2009b). The nature of ESM measurements may benefit research aimed at identifying scars. Thus, in the search for scars, future research should use methods able to detect changes in subtle and context-dependent subject characteristics.

\section{Two processes: scarring due to depressive symptoms and due to the causes of depressive symptoms}

Another question that needs to be resolved is whether the hypothesized scarring is caused by the depressive symptomatology itself or by the triggers that caused the depressive symptoms in the first place, such as stress exposure, or by a combination of these factors. To date, studies that have investigated the existence of scar effects did not differentiate between the possible scarring effects of the depressive symptoms or pre-existing conditions - such as extreme stress - before the onset of the depressive episode. Both the experience of stress and experience of depressive symptoms may produce scar effects. Post and colleagues (Post, 1992) refer to two types of sensitization mechanisms, one related to the stressor and one to the experience of an affective episode. Psychosocial stress may induce a cascade of neurobiological events that have long-lasting consequences such as altered gene expression. In this way, the individual may end up more vulnerable and exhibit altered behavioral responses to stress because of the sensitization by previous stressors (Post, 1992).

Alternatively or perhaps additionally, the experience of an affective episode may leave behind traces that predispose to further episodes as argued by Post and co-workers (Post, 1992). A related view, developed from the above sensitization theory, that better fits with the continuous phenotype of depression and at the same time constitutes an integration between a cognitive and neurobiological perspective on depression, was proposed by Segal and colleagues (Segal, Williams, Teasdale, \& Gemar, 1996), based on the associative network theory. According to this theory, a network of inter-related nodes exists in which activation may spread from node to node. Concepts may bring to consciousness other closely related concepts via the spreading activation within this network. The likelihood of cognitive patterns being activated is dependent on the frequency of past usage analogue to biological mechanisms of neuronal learning. Based on this theory, it is argued that people strengthen their negative thought patterns every time they use them. The experience of severe adverse experiences may create a network of intensely negative "depressogenic" themes that is 
related to the affect experienced. Thus, each time an individual experiences low mood (s)he is prone to activate other elements within the negative concepts network which will again strengthen - or sensitise - the paths of this network, making it more accessible each time a moment of low mood presents itself in the future (Segal, et al., 1996). This view is compatible with the assumption that depressive symptoms over the whole continuum of depression may contribute to scar effects. A recent study showed that developmental stress exposures increased later daily-life stress sensitivity (conceptualised as negative affective response to minor daily stressors) (Wichers, et al., 2009c) which fits within the sensitization theory and the cognitive view on sensitization in depression. For example, early adversity may induce negative cognitive schemes (a scar due to a stressor) that become more readily activated each time they are used, i.e. at the time of experiencing stressors or mild dysphoric states (continuously increasing scar due to negative affective states). As a result, small daily-life stressors may activate negative cognitive schemes with progressively lower thresholds, so that the subject responds with progressively stronger negative affect to daily-life stressors.

As mentioned before, the higher the daily-life stress sensitivity, the stronger its association with future increase in depressive symptoms and episodes of depression (Segal, et al., 1996; Wichers, et al., 2009b; Wichers, et al., 2009c). A plausible biological substrate for the effect of early adversity on the development of a negative scheme is amygdala reactivity. Heightening of amygdala reactivity is known to follow traumatic experiences (Ganzel, Casey, Glover, Voss, \& Temple, 2007; Ganzel, Kim, Glover, \& Temple, 2008) and this increase is associated with automatic negative evaluation response tendencies and a negative bias in the interpretation of faces (Beck, 2008; Dannlowski, et al., 2007a; Dannlowski, et al., 2007b).

In conclusion, both stressors and negative mood states may be able to produce scars that are relevant to the vulnerability for depression. Additionally, these processes may work together in producing vulnerability and are likely gradually inducing an increased amount of scar over periods of time. However, no previous studies examining the existence of scars have tried to disentangle the two processes. Another issue is whether a threshold may exist after which scarring consolidates. Some evidence exists that for example the process of kindling (evident from the progressively reduced association between major life events and risk of each further depressive episode) involved in the risk for new depressive episodes reaches consolidation at a certain point after which the amount of acquired vulnerability stabilizes (Kendler, Thornton, \& Gardner, 2000). 
Future studies examining scar effects should try to take into account these processes in order to unravel the complex mechanisms of the progressively increasing vulnerability for depression due to scar effects.

\section{The issue of reversibility - can we heal the scar?}

Finally, the question arises whether a scar is reversible or not. The name 'scar' implies a permanent increase in vulnerability. However, biological plausibility may be higher for a 'scar' or vulnerability concept that has dynamic properties, something that can increase and decrease under certain circumstances. In recent years, the concept of epigenetics, evidence that genetic function is not static but plastic, has also gained attention within the field of psychopathology research. The idea that epigenetic effects also apply to psychopathology may help identifying evidence for the biological plausibility of reversibility of scars. Gene function can be altered following adverse circumstances and may become expressed as a change in behavior (scarring effect). Recent studies have reported associations between epigenetic status and mood or depression (Oberlander, et al., 2008; Philibert, et al., 2008; Poulter, et al., 2008). Although speculative, it may be parsimonious to hypothesize that certain environmental influences may also change genetic epi-mutations back into their default states. This may subsequently reduce the amount of scarring, the risk for mood abnormalities, and formation of psychopathology. However, to date this has not been studied.

There is biological plausibility for reversibility not only at the level of genetics but also at the level of neuronal mechanisms of learning. For instance, in the example described earlier, based on the associative network theory, one would predict that not using the connections between negative concepts will weaken the strength of the associations, increase the threshold for activation and thus decrease this type of vulnerability. Not using specific (negative) network associations may be a very active process which can reduce previously developed scars.

In addition, evidence from a recent study (Wichers, et al., 2007a) is suggestive of scar reversibility. The dynamic person-context interaction concept of daily-life stress sensitivity can be seen as scar of past stressors and dysphoric states according to the new definition proposed in this paper. It gradually increases following early stress exposures (Wichers, et al., 2009c), is associated with depressive symptoms (Wichers, et al., 2007b) and predicts the development of future new affective symptoms and episodes of MDD (Wichers, et al., 2009b). For this potential scar, evidence exists that other factors may decrease its effects. It has been found that the experience of positive emotions during stressors decreases the level of stress sensitivity (Wichers, et al., 2007a). Moreover, 
positive emotions neutralize the expression of genetic risk associated with increased daily-life stress sensitivity (Wichers, et al., 2008b). Thus, there are mechanisms that may decrease the impact of potential scars.

A dynamic view on scars encourages a more optimistic and less deterministic view on vulnerability. In addition, it emphasizes the role of resilience in depressive disorder: mechanisms implicated in positive traits and strategies of the subject that are relatively independent from vulnerability mechanisms, may have crucial importance for diminishing scars and preventing (recurrence of) depressive symptomatology.

\section{SCARS AND TREATMENT OF DEPRESSION}

Knowledge of reversibility of scars is highly relevant to the treatment of MDD. As the scars of previous experienced stress or negative affective states form a vicious circle by which people become more and more depressed (and thus develop new scars), treatments for MDD should also be focussed on reduction of scarring effects. Only by disruption of this vicious cycle can the long-term course of MDD be bent into a more favourable direction. This makes the discovery of the basic mechanisms involved in scarring a priority in depression research.

Some treatments already may work by impacting on the processes of scarring. A new treatment, 'mindfulness-based-cognitive-therapy' (MBCT) (Teasdale, et al., 2002; Teasdale, et al., 2000), attempts to teach individuals to focus attention on the presence and to let go of negative thoughts about past experiences or future worries. Subjects with sensitised negative cognitive schemes may profit from this approach as it helps them to detach from thoughts that activate these schemes. By actively avoiding the spreading of activation through the negative cognitive schemes, the strength of these paths will eventually diminish. Thus, people will desensitise their network of negative cognitions, and thus their scar, that caused negative emotions to flourish (Sheppard \& Teasdale, 2004). Cognitive behavioral therapy (CBT) may also be effective partly through the desensitization of the network of negative cognitions. Segal and colleageus (Segal, Gemar, \& Williams, 1999) showed that after negative mood induction, patients successfully treated with CBT exhibited significantly less dysfunctional cognitions than those successfully treated with pharmacotherapy. Thus, CBT may be able to undo relevant underlying cognitive scars which were not affected by medication treatment.

Another future application of scar research to clinical practice is the assessment of change of potential scars during standard therapy. Dynamic 
person-context phenotypes (hereafter: Experience Sampling Method [ESM] phenotypes) such as 'stress sensitivity' are, as argued above, potential scars and can be measured using ESM technology. The assessment of treatment response in terms of scar changes may induce new possibilities. Instead of assessing treatment response in terms of remission and recovery, for which monitoring of change in depressive symptoms is sufficient, the additional assessment of 'scar change' may be useful as an indicator of risk for future depressive symptomatology. A recent study showed that in remitted depressed subjects, ESM phenotypes, independently from baseline depressive symptoms, contribute to the prediction of future symptoms and relapse (Wichers, et al., 2009b). Assessment of a reduction in the mechanisms underlying the continuation of symptoms - the scars - during treatment may constitute a complementary way to assess treatment response (in addition to assessment of decrease in symptoms only) and may increase power to predict future course of depression.

\section{CONCLUDING REMARKS}

The progressively growing risk of depression relapse with a higher number of past experienced episodes of depression provides strong support for the theory that scars related to past experiences may increase a person's level of vulnerability. However, studies have generally not been successful in detecting scars and their mechanisms. We propose a new approach to examine the concept of a 'scar' that (i) is based on a dimensional view on depression, i.e that scars may develop following depressive symptoms at all points of the continuum from low to high depression, ii) uses methods that take into account the dynamic interplay between the person and its context to be able to detect subtle changes in personenvironment interplay, (iii) differentiates between scars following depression and scars following that what caused the depression such as stress exposure, and (iv) holds a dynamic view of the concept of 'scar' in that it hypothesizes that scars can wax and wane, stimulating research into mechanisms of resilience.

If scars are conceived as continuously and gradually developing from interactions between the effects of lingering traces of stress and the experience of low mood, it is implied that scars operate at a sub-clinical as well as a clinical level. Furthermore, starting from the sub-clinical level, scars may keep growing - making people more and more vulnerable - and create a vicious circle. The above view necessitates a new approach in the examination of scars that may stimulate the finding of new entries in the puzzle of the development of vulnerability and resilience. Moreover, this approach may provide insights that help to develop new 
therapies for MDD aimed at decreasing scar(s) and complementary assessment methods for evaluation of treatment response. 


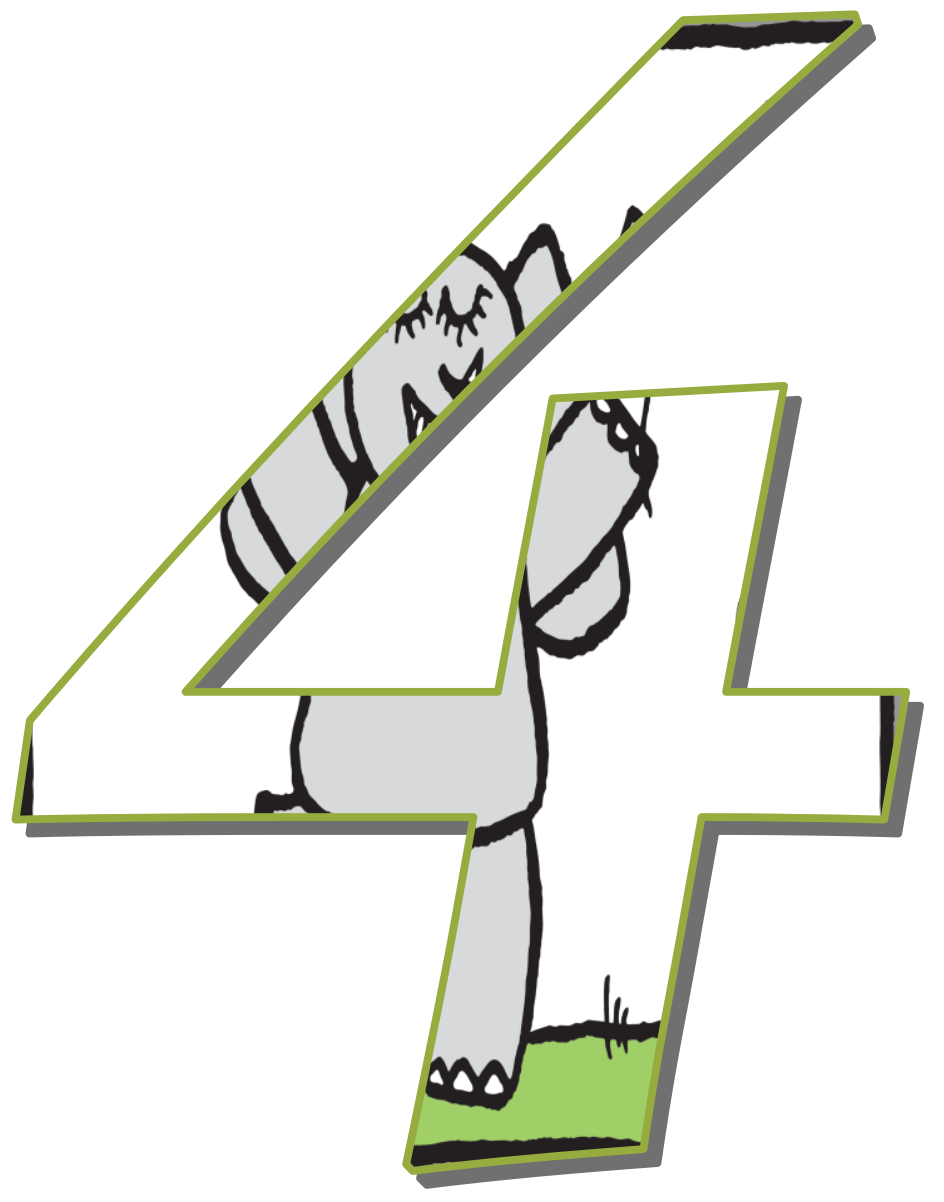

Transition from stress sensitivity to a depressive state: A longitudinal twin study 


\section{ABSTRACT}

Background: Daily-life stress sensitivity is associated with depression but prospective data are lacking.

Aims: To examine associations between baseline ecological daily-life stress sensitivity and follow-up depression and to identify genetic and non-genetic factors moderating transition from stress sensitivity to depression.

Method: Daily-life stress sensitivity was assessed at baseline in twins $(n=502)$. One baseline and four follow-up measurements of SCL-90 depressive symptoms and negative life events were collected, as well as interview-based DSM-IV diagnosis at baseline and last follow-up. Hypothesised genetic markers were determined.

Results: Baseline stress sensitivity was associated with increased follow-up depressive symptoms and risk for major depressive disorder. Genetic liability and aetiological independence from major life events increased the probability of transition from stress sensitivity to depression

Conclusions: Onset of depression is attributable to pre-onset ecological measures of stress sensitivity, particularly when genetic liability is high and individuals have reached a stage where the influence of competing environmental causes is low.

\section{Published as:}

Wichers, M., Geschwind, N., Jacobs, N., Kenis, G., Peeters, F., Derom, C., et al. (2009). Transition from stress sensitivity to a depressive state: Longitudinal twin study. The British Journal of Psychiatry, 195(6), 498-503. doi: 10.1192/bjp.bp.108.056853 
Recent findings suggest that genetic liability to depression is in part expressed as the tendency to display NA in response to minor stressors in the flow of daily life (Wichers, et al., 2007b). Thus, a causal pathway from genetic risk for daily-life stress sensitivity to the development of affective symptoms may be hypothesised. Additional support for a causal pathway from daily-life stress sensitivity to the development of depression can be obtained by assessing the within-subject association between baseline daily-life stress sensitivity and the development of future affective symptomatology. The current report examines this issue in a large general population female twin sample, using Experience Sampling Methodology (ESM) (Csikszentmihalyi \& Larson, 1987) and prospective dimensional and diagnostic follow-up measurements of depression. In addition, since not all subjects with high levels of stress sensitivity will develop depression, factors that may moderate transition to depression were examined. It was hypothesised that indirect measures of genetic risk and direct molecular genetic variation in the BDNF val ${ }^{66}$ met and 5-HTTLPR polymorphisms on the one hand (Bergstrom, Jayatissa, Mork, \& Wiborg, 2008; Caspi, et al., 2003; Hayden, et al., 2008; Kaufman, et al., 2006), and occurrence of negative life events on the other moderate transition from experiencing increased daily-life stress sensitivity to future affective symptomatology.

\section{METHODS}

\section{Subjects}

The study sample consisted of 621 individuals, aged between 18 and 46 years, from a general population twin sample from Flanders (Belgium). Two-hundred and eighteen twin pairs were recruited from the East-Flanders Prospective Twin Survey. This population-based survey has prospectively recorded all multiple births in the province of East-Flanders since 1964 (Derom, et al., 2002; Loos, Derom, Vlietinck, \& Derom, 1998). Zygosity was determined as described elsewhere (Jacobs, et al., 2006). The project was approved by the Local Ethics Committee and all participants gave written informed consent. The sample was female only, given the probability of qualitative differences in the type of environmental stressors that are associated with depression in men and women (Kendler, Thornton, \& Prescott, 2001), and sex-specific genetic factors for both neuroticism and depression (Fanous, Gardner, Prescott, Cancro, \& Kendler, 2002).

\section{Experience Sampling Method}

ESM is a structured diary technique to assess subjects in their daily living environment, which has been validated for the use of studying the immediate 
effects of stressors on mood (Csikszentmihalyi \& Larson, 1987; Delespaul, 1995; DeVries, 1992; Jacobs, et al., 2005). Subjects received a digital wristwatch and a set of ESM self-assessment forms collated in a booklet for each day. The wristwatch was programmed to emit a signal ("beep") at an unpredictable moment in each of ten 90-minute time blocks between 7:30 and 22:30, on five consecutive days. After each beep, subjects were asked to stop their activity and to fill out the ESM self-assessment forms previously handed to them, collecting reports of thoughts, current context (activity, persons present, and location), appraisals of current situation and mood (for further description see Wichers, et al., 2007b).

\section{Procedure}

The sample was assessed at five time points, including a baseline (TO) and four follow-up measurements (T1-T4). The average number of days between T0 and T1 was 132, between T1 and T2 91, between T2 and T3 116 and between T3 and T4 91. The first interview was at the home of the individuals, follow-up data were collected using questionnaires and telephone interviews. All interviews were performed by trained research psychologists or graduate psychological assistants.

\section{Measurements}

\section{Affective symptoms}

The Structured Clinical Interview for DSM-IV axis I disorders (SCID-I) (First, Spitzer, Gibbon, \& Williams, 1995) was administered in order to obtain current and life time diagnosis of major depressive disorder. The SCID interview was conducted at T0 and T4. At all time points, subjects filled in the Symptom Checklist (SCL-90R) (Derogatis, Rickels, \& Rock, 1976). SCL-90R depression and anxiety scales were averaged for each measurement occasion, resulting in a combined score reflecting affective symptoms (hereafter: affective symptoms).

Recent Negative Life events

At each time point, an inventory of recent life events was made, based on the event list of the Interview of Recent Life Events (IRLE) (Paykel, 1997). Subjects reported if one of 61 events happened in the last six months (at baseline) and since the last measurement occasion (at follow-up) and rated their impact on a five-point scale (from $1=$ very pleasant to $5=$ very unpleasant). For further description see Jacobs and colleagues (Jacobs, et al., 2006). Congruent with earlier analyses in this sample (Jacobs et al, 2006), events rated as unpleasant (i.e. score of 4 , "unpleasant" or score of 5 "very unpleasant) defined life event occurrence 
and a continuous exposure variable was made representing the number of such unpleasant events befalling the individual over the last six months.

Since previous studies have shown that experience of early adversity may potentially confound the association between recent negative life events and risk for depression (Wichers, et al., 2009c), childhood adversity was measured using the shortened version (Bernstein, Ahluvalia, Pogge, \& Handelsman, 1997) of the 70-item CTQ questionnaire (Arntz \& Wessel, 1996; Bernstein, et al., 1994). At the request of the Twin Registry, the most explicit items concerning sexual and physical abuse were omitted; less explicit items were retained.

\section{Daily-life NA and stress appraisals}

Measures of stress and NA were collected at each beep in the ESM protocol at T0. In order to measure ESM event-related stress (STRESS), subjects were asked to report the most important event that happened between the current and the previous beep. This event was subsequently rated on a 7-point bipolar scale (-3 = very unpleasant, $0=$ neutral, $3=$ very pleasant). Responses were recoded to allow high scores to reflect stress ( $-3=$ very pleasant, $0=$ neutral, $3=$ very unpleasant). ESM NA was the mean of 6 mood adjectives (I feel 'insecure', 'lonely', 'anxious', 'low', 'guilty' and 'suspicious') rated on 7-point Likert scales.

\section{Genotyping}

Placental tissue for DNA analysis was available for 156 participants, blood samples for 14 , and buccal cell samples for 208 , using a sterile swab specifically designed for the collection of buccal cell samples for DNA testing (Omni Swabs; Whatman PLC, Brentford, England).

Genotyping of the 5-HTTLPR was carried out as described previously (Jacobs, et al., 2006). BDNF Val ${ }^{66}$ Met (rs6265) genotyping was done by KBiosciences (Herts, UK, http://www.kbioscience.co.uk), using an assay based on Taqman allelic discrimination. For every monozygotic (MZ) twin in the sample with genotypic data, the same genotypic data were included for the co-twin, assuming identical genotypes.

\section{Statistical analysis}

Previous studies by our group examined daily-life stress sensitivity as the effects of minor stressors (STRESS) as exposure variable on NA in the flow of daily life as response variable (Myin-Germeys, van Os, Schwartz, Stone, \& Delespaul, 2001; Wichers, et al., 2007b). In the current study, however, the response variable was affective symptomatology and daily-life stress sensitivity represented the exposure variable, the effect of which on affective symptomatology needs to be modelled statistically. Therefore, the variable 'stress sensitivity' was constructed 
from the ESM observations reflecting the within-subject average effect size of STRESS on NA (based on an average of 27.7 effect sizes per subject). Furthermore, a variable was created representing the average negative life event score measured over T1-T4. The variable 'co-twin depression' was created to reflect familial vulnerability to depression and was defined as a past diagnosis of depression in the co-twin. In order to make maximal use of the five measurement occasions of affective symptoms, data were analysed in the long format using multi-level analysis techniques (XTMIXED command in STATA 10 (StataCorp, 2007)). Data from multiple follow-up observations (level 1) were clustered within participants (level 2), who were part of twin pairs (level 3). For the analyses with follow-up DSM-IV diagnosis of depression as response variable, multilevel logistic regression analyses were conducted using the command XTGEE in STATA 10 (Snijders \& Bosker, 1999). In these analyses, subject observations (level 1) were clustered within twin pairs (level 2).

First, the effect of baseline stress sensitivity on follow-up affective symptoms (T1-T4) was examined, corrected for affective symptoms at TO. Similarly, the association between stress sensitivity and follow-up DSM-IV diagnosis of depression at T4 was examined, but here, additionally, all subjects with a DSM-IV diagnosis of depression at TO were excluded. In case of significant linear effects, dose-response in the association was examined by dividing the distribution of stress sensitivity by its tertiles using the XTILE command, thus creating tertile groups.

Second, interaction effects were examined between stress sensitivity on the one hand and i) familial risk (co-twin depression) ii) indirect genetic risk (i.e. examining $M Z$ and $D Z$ genetic contrasts using the zygosity $x$ co-twin depression interaction term) iii) direct genetic variation using 5 -HTTLPR and BDNF val ${ }^{66}$ met genetic polymorphisms and iv) post-baseline negative life events, on the other. These interaction effects were examined both for the dimensional outcome of depression and DSM-IV diagnosis of depression measured at T4. All interaction analyses were corrected for SCL-90 score of affective symptoms at T0. In those with follow-up DSM-IV diagnosis of major depression as dependent variable, subjects with current DSM-IV diagnosis of depression at baseline were excluded. In the analyses using co-twin measures of familial risk or indirect genetic risk, sensitivity analyses were performed with additional correction for baseline current or past DSM-IV diagnosis of depression. Analyses including negative life events were corrected for the continuous score of early adversity as measured with the shortened version of the CTQ.

Variables were standardised in order to report standardised effect sizes. 


\section{RESULTS}

\section{Subject characteristics}

The total sample consisted of 621 white subjects, of which 610 participated in the ESM procedure. Thirty-one subjects were excluded because they had missing or too few valid ESM self-reports. Another 49 subjects were excluded due to missing ESM data and 28 due to insufficient ESM data for the construction of the withinsubject averaged effect size of STRESS on NA. This resulted in a dataset of 502 subjects that were part of 250 different twin pairs (152 were MZ twins, 97 were DZ twins and one pair was of unknown zygosity) and that included 36 non-twin sisters (see Table 1 for sample description). Follow-up data with respect to followup affective symptoms were present for 481 subjects at T1, 476 at T2, 450 at T3 and $443(88 \%)$ at T4. For $391(78 \%)$ subjects, SCID DSM-IV diagnoses were available at T4. The distribution of genotypes of the polymorphisms of the 5HTTLPR and BDNF val ${ }^{66}$ met was in Hardy-Weinberg equilibrium (BDNF Val/Val: 269, Val/Met: 112, Met/Met: $21 ; \chi^{2}=3.8, \mathrm{df}=1, \mathrm{p}>0.05 ; 5$-HTTLPR: LL: 121, LS: 158, SS: 76; $\left.\chi^{2}=3.2, \mathrm{df}=1, \mathrm{p}>0.05\right)$. Results from analyses regarding the Met/Met variant were not interpreted as too few subjects $(n=21)$ were included.

At baseline, eighty-nine out of 502 (17.7\%) subjects ever had fulfilled DSMIV criteria for major depression (hereafter: history of major depression) and 51 out of 391 (13\%) developed a DSM-IV diagnosis of depression between baseline and T4, of which 14 had a baseline history of major depression. Sixteen percent of those with high (highest 33\%) daily-life stress sensitivity and without history of major depression developed major depression in the period between baseline and T4, compared to $10 \%$ of those with average and only $6 \%$ of those with low daily-life stress sensitivity. Almost half (46\%) of those that developed a major depression had displayed daily-life stress sensitivity at the level of the highest $33 \%$ at baseline. People with a baseline history of major depression showed significantly increased daily-life stress sensitivity $(\beta=0.35, p=0.002)$. A significant association was present between the variables making up the baseline stress sensitivity $X$ post-baseline negative life events interaction term $(\beta=0.08, p=$ $0.043)$. No other associations were present between variables in other interaction terms. 
Table 1

Sample Characteristics.

\begin{tabular}{lc}
\hline Age in years: mean (SD) & $27.4(7.4)$ \\
Average baseline SCL-90 item score: mean (SD) & $1.44(0.4)$ \\
Average follow-up SCL-90 item score: mean (SD) & $1.35(0.4)$ \\
Average number of post-baseline negative life events: mean (SD) & $3.8(3.8)$ \\
Education, \% & \\
college or university degree & 66 \\
secondary education & 33 \\
primary education only & 1 \\
Employment, \% & \\
employed & 64 \\
student & 32 \\
unemployed & 2 \\
homemaker & 2 \\
sick leave & 1 \\
\hline
\end{tabular}

Note. SCL-90-R = Symptom Checklist - 90 - Revised

\section{Baseline daily-life stress sensitivity and follow-up affective symptoms}

Baseline daily-life stress sensitivity was significantly associated $(\beta=0.16, p=0.01$; corrected for baseline) with follow-up affective symptoms. Additionally, a doseresponse association was apparent from average compared to low stress sensitivity $(\beta=0.43, p=0.005)$ and high compared to low stress sensitivity ( $\beta=$ $0.46, p=0.003$, see Figure $1 \mathrm{a})$. The regression coefficient linear trend was significant $(\beta=0.24, p=0.003)$. Furthermore, baseline stress sensitivity was significantly predictive of follow-up major depression in subjects without current major depression at baseline $(O R=1.34, p=0.048)$. Again, a dose-response association was apparent from average compared to low stress sensitivity (OR = $1.10, p=0.82)$ and high compared to low stress sensitivity $(O R=1.75, p=0.16$; see Figure $1 \mathrm{~b}$ ). The $\beta$ linear trend, however, was not statistically significant ( $\beta=$ $1.34, p=0.14$ ). A post-hoc analysis showed that excluding additionally those with past diagnosis of depression at baseline even further strengthened the association between stress sensitivity and follow-up diagnosis of depression $(\mathrm{OR}=1.43, p=$ 0.030). 
(a)

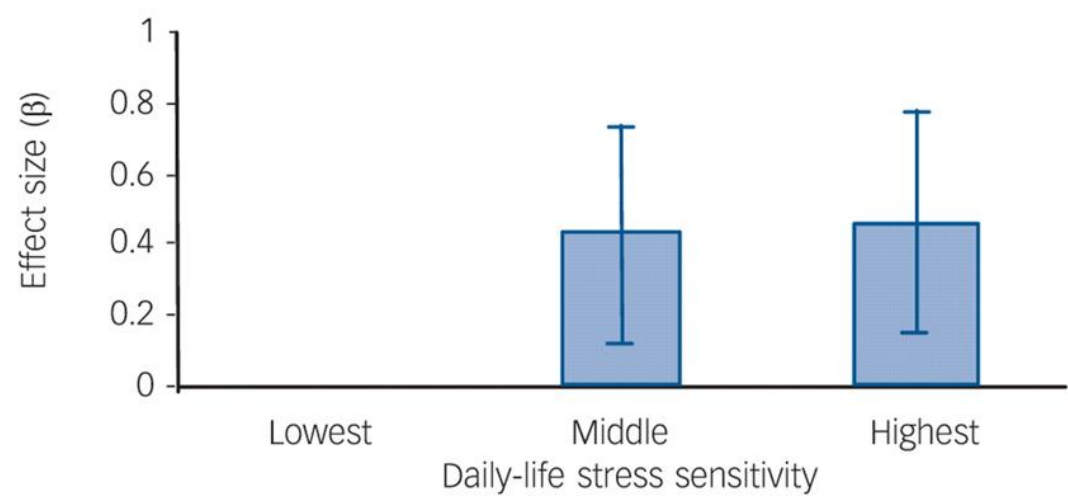

(b)

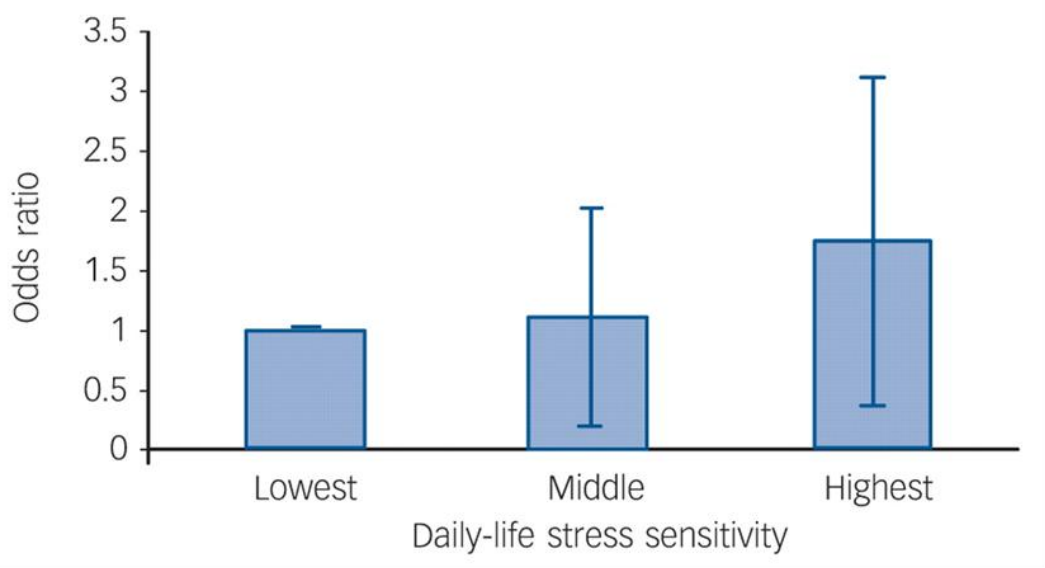

Figure 1. Dose-response association between daily-life stress sensitivity, divided into tertile groups, and subsequent depression.

(a) Depressive symptoms: standardised effect sizes ( $\beta$ ) for depressive symptoms at follow-up; (b) major depressive disorder: standardised effect in odds ratios (ORs) for a diagnosis of DSM-IV depression at last follow-up assessment. Error bars depict 95\% confidence intervals.

Moderation of the association between daily-life stress sensitivity and follow-up depressive symptomatology

Familial vulnerability for depression

There was no interaction between co-twin depression and baseline stress sensitivity in the model of follow-up affective symptoms $\left(\chi^{2}=0.88, p=0.35\right)$. However, in probands with co-twin depression, the association was greater ( $\beta=$ 0.29 ) than in those without $(\beta=0.14)$. A sensitivity analysis, additionally corrected for a baseline history of major depression, showed similar effect sizes (co-twin depression: $\beta=0.26$ and no co-twin depression: $\beta=0.12$; interaction $\chi^{2}=0.74, p=$ 
0.39). Similarly, no significant interaction effect was found in the model of followup major depression $\left(\chi^{2}=0.04, \mathrm{OR}=0.93, p=0.84\right)$.

\section{Genetic vulnerability for depression}

The three-way interaction effect $\left(\chi^{2}=4.42, p=0.036\right)$ between co-twin depression and baseline stress sensitivity in the model of follow-up affective symptoms was significantly greater within $\mathrm{MZ}$ twin pairs $(\beta=0.039, p=0.047)$ than $\mathrm{DZ}$ twin pairs $(\beta=-0.024, p=0.30)$. Correction for proband history of DSM-IV depression revealed similar results (interaction: $\chi^{2}=4.57, p=0.032 ; \mathrm{MZ}: \beta=0.038, p=0.051$; DZ: $\beta=-0.026, p=0.25)$. No significant interaction was found in the model of follow-up major depression $\left(\chi^{2}=2.17\right.$, OR $\left.=0.90, p=0.14\right)$.

Direct genetic markers associated with depression: HTTLPR and BDNF Val ${ }^{66}$ Met polymorphisms

HTTLPR variation significantly moderated the baseline stress sensitivity effect on follow-up affective symptoms (long-short variant [LS] compared to long-long [LL] $\chi^{2}=4.13, \beta=-0.34, p=0.042$; short-short [SS] variant compared to long-long: $\chi^{2}=$ $0.02, \beta=0.03, p=0.89)$. Also, variation in the BDNF Val ${ }^{66} \mathrm{Met}$ polymorphism moderated the effect of baseline stress sensitivity on follow-up affective symptoms (val/met compared to Val/Val: $\chi^{2}=4.96, \beta=0.33, p=0.026$ ). No significant moderation by these polymorphisms was found in the model of followup major depression.

Recent negative life events (post-baseline)

Corrected for baseline depressive symptoms and early adversity, a significant effect of post-baseline negative life events on follow-up depressive symptoms was apparent $(\beta=0.74, p<0.001$ ) as well as a significant effect on follow-up major depression $(O R=1.43, p=0.014)$. In addition, a significant interaction was apparent between post-baseline negative life events and daily-life stress sensitivity in the model of follow-up depressive symptoms $\left(\chi^{2}=5.67, \beta=-0.12, p=\right.$ $0.017)$. Although tertile group analyses also showed that the effect of negative life events diminished with increased baseline stress sensitivity, these stratified analyses did not yield significant results (average compared to low stress sensitivity: $\beta=-0.02, p=0.92$; high compared to low stress sensitivity: $\beta=-0.10, p$ $=0.48$; dose-response trend: $\beta=-0.06, p=0.38$; see Figure 2 for absolute effect sizes of negative life events per level of stress sensitivity). There was no significant moderating effect of negative life events in the model of follow-up major depression. 


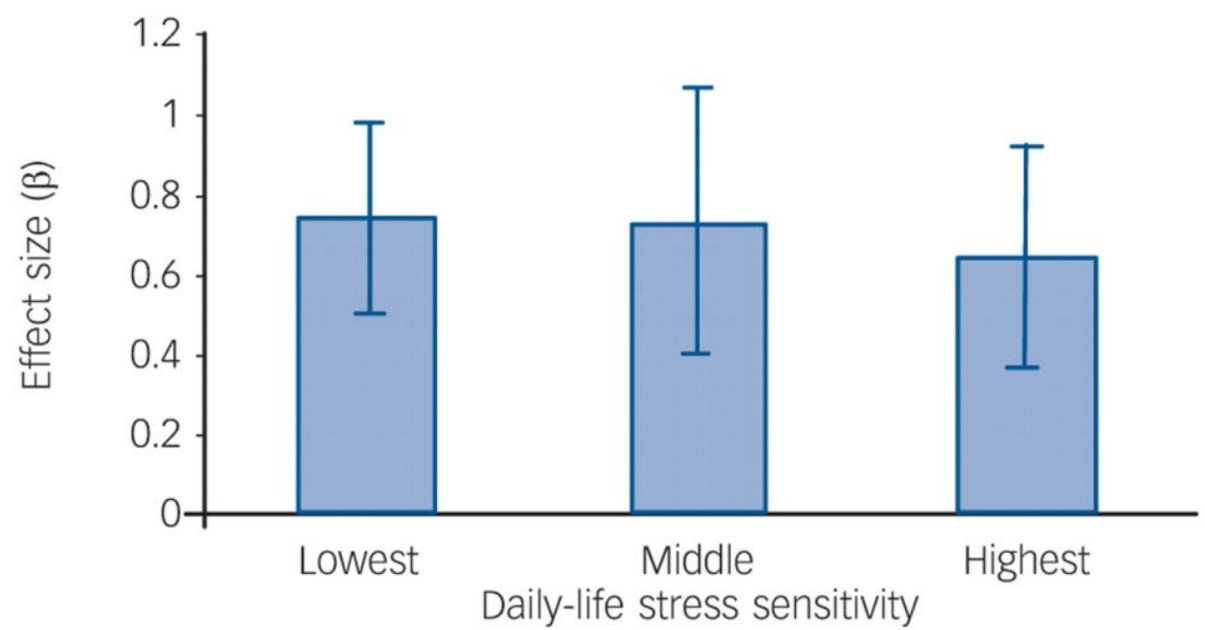

Figure 2. Post-baseline negative life events interaction with baseline daily-life stress sensitivity in predicting the development of depressive symptoms.

The standardised effect sizes $(\beta)$ of negative life events on follow-up depressive symptoms are shown separately for each tertile group of daily-life stress sensitivity. Error bars depict $95 \%$ confidence intervals.

\section{DISCUSSION}

Daily-life stress sensitivity predicted future increase in affective symptoms - shifts to higher positions on the depression continuum (Kendler \& Gardner, 1998) - as well as transition to a full-blown DSM-IV depressive disorder in subjects without any history of DSM-IV depressive disorder. In addition, results were compatible with a dose-response relationship: the higher the stress sensitivity, the greater the association with later affective symptoms. However, shifting from low to average stress sensitivity predicted the largest increase in risk, while a shift from average to high made a minor extra contribution. The dose-response association did not reach significance for follow-up diagnosis of depression. This is the first study showing associations between stress sensitivity in daily life and future depressive symptomatology. Replication is necessary before considering the findings conclusive.

\section{Role of genetic moderators}

The findings (based on both indirect and direct genetic measures) support the hypothesis that genetic factors moderate the effect of daily-life stress sensitivity on a future upward shift along the depression continuum. Indirect genetic 
vulnerability - sharing $100 \%$ of the genes with a subject with a history of depression compared to sharing only $50 \%$ - was shown to increase the effect of daily-life stress sensitivity on future depressive symptomatology. Also, variations in polymorphisms associated with depression are suggestive of genetic influence. Subjects with a Met allele in the BDNF Val ${ }^{66}$ Met polymorphism, compared to those without, were at increased risk for transition of stress sensitivity to the development of new affective symptoms. The Met allele - associated with lower BDNF serum levels than the Val allele - has been associated with stress-related outcomes and meta-analyses, albeit not all, suggest association between BDNF and depression at various phenotypic levels (Brunoni, Lopes, \& Fregni, 2008; Sen, Duman, \& Sanacora, 2008; Verhagen, et al., 2008). In addition, recent studies that examined possible moderation of BDNF $\mathrm{Val}^{66} \mathrm{Met}$ of the effect of stress on depressive symptoms show a similar increased risk associated with the Met allele (Aguilera, et al., 2009; Gatt, et al., 2009; Kaufman, et al., 2006). Therefore, the current findings agree with existing literature and extend it to minor stressors in the flow of daily life.

In addition, variation in the 5-HTTLPR was associated with increased risk of transition to symptomatic affective dysregulation: the SL variant appeared protective compared to both the SS and the LL variants. The literature is inconsistent regarding the effects of the 5-HTTLPR in relation to depression. Both SS and LL variants have been shown to increase risk (Araya, et al., 2008; Hayden, et al., 2008; Laucht, et al., 2009). Therefore, the current finding should be interpreted cautiously and needs replication. To reduce noise and increase the probability of true positive findings, future research should enrich studies with haplotype analyses or, if possible, data on epigenetic variation conveying information on the transcriptional activity of a particular segment of DNA.

\section{Role of environmental moderators}

Environmental factors also moderate the effect of stress sensitivity. Conform previous literature (Bouma, Ormel, Verhulst, \& Oldehinkel, 2008; Comijs, et al., 2007) there were main effects of negative life events on both dimensional and dichotomous measures of depression. However, negative life events negatively moderated the effect of stress sensitivity on follow-up affective symptoms. Thus, the higher the level of stress sensitivity, the lower the influence of negative life events on future depression. A possible interpretation of this result may be found in findings reported by Kendler and colleagues (Kendler, et al., 2000), who showed that the influence of life events on depression decreased with multiple past episodes of depression. The authors proposed 'kindling' or 'sensitization' as the cause of this phenomenon leading to more autonomous onsets of depression not 
related any more to major stressful events. Daily-life stress sensitivity has been shown to reflect sensitization following past stress exposure (Wichers, et al., 2009c). It may be hypothesised that individuals with increasing number of past episodes of depression will develop increased daily-life stress sensitivity - which indeed was the case in the current sample (see subject characteristics) and that negative life events now have to "compete" with daily-life stress sensitivity for new onsets of depression. This would support the theory - as discussed by Monroe \& Harkness (2005) - that stressors do no loose the capacity to trigger depression, but, on the contrary, that even small daily-life stressors already are capacitated to cause depression, thereby overtaking, in terms of attributable fraction, onsets caused by infrequent major life stressors.

\section{Daily-life stress sensitivity as an intermediate phenotype of depression}

As mentioned in the introduction, there is evidence that ecological daily-life stress sensitivity represents the behavioral expression of genetic risk for depression (Wichers, et al., 2007b). The current findings indicated that additionally the mechanisms of stress sensitivity mediate onset of depression, i.e. are causal. Factors that substantiate the hypothesis of causality are (i) temporal order, i.e. the exposure preceded the disease outcome, (ii) the presence of a dose-response association, (iii) biological plausibility and (iv) consistency with other research findings (Bradford-Hill \& Proceedings of the Royal Society of Medicine, 1965). Although causality is something that can never be concluded with certainty, the findings presented here support the notion that daily-life stress sensitivity causally influences the risk for future depressive symptomatology. Furthermore, the strength of the association between the endophenotype daily-life stress sensitivity and depression appears to be dependent on both genetic and environmental factors, illustrating the complexity of the behavioral disorder that is called major depression and the need for studies that take into account these complex interacting mechanisms.

\section{Limitations}

There was a certain amount of drop-out over the follow-up measurements that may have introduced bias. However, drop-out was relatively small. Bias due to differential drop-out is unlikely since it is not to be expected that depressed subjects at follow-up with low baseline stress sensitivity dropped out at a different rate - or higher rate - than those with high baseline stress sensitivity.

The study population was large. There was thus enough power to detect differences in depressive symptoms using a dimensional scale. However, for the analyses using the dichotomous measure 'DSM-IV diagnosis of depression' as 
outcome variable, especially for the power-hungry interaction analyses, low power may have contributed to inconclusive findings.

Another issue is that exposure to life events is not necessarily a true environmental factor (Eaves, Silberg, \& Erkanli, 2003). It has been shown that genetic factors influence exposure to life events. Therefore, findings should be interpreted accordingly. Finally, measurements of negative life events were based on self-reports as opposed to structured interviews like the Life Events and Difficulties Schedule (LEDS), increasing the possibility of retrospective bias and more error in the timing of event occurrence. Although negative life events were not rated specifically with regard to level of contextual threat, the rating of negative emotional attribution in the IRLE arguably constitutes a valid way of identifying those occurrences most likely to shape the risk of future depression.

\section{Acknowledgements}

This research was supported by the Dutch Organisation for Scientific Research; the Fund for Scientific Research, Flanders and Twins, a nonprofit association for scientific research in multiple births (Belgium) (to the East Flanders Prospective Survey); and the EU Framework 6 Integrated Project NewMood (LSHMCT-2004-503474) (to Dr Kenis).

We thank all twins for their cooperation. Dr MC Wichers was supported by the Dutch Organisation for Scientific Research (VENI grant nr 916.76.147). 


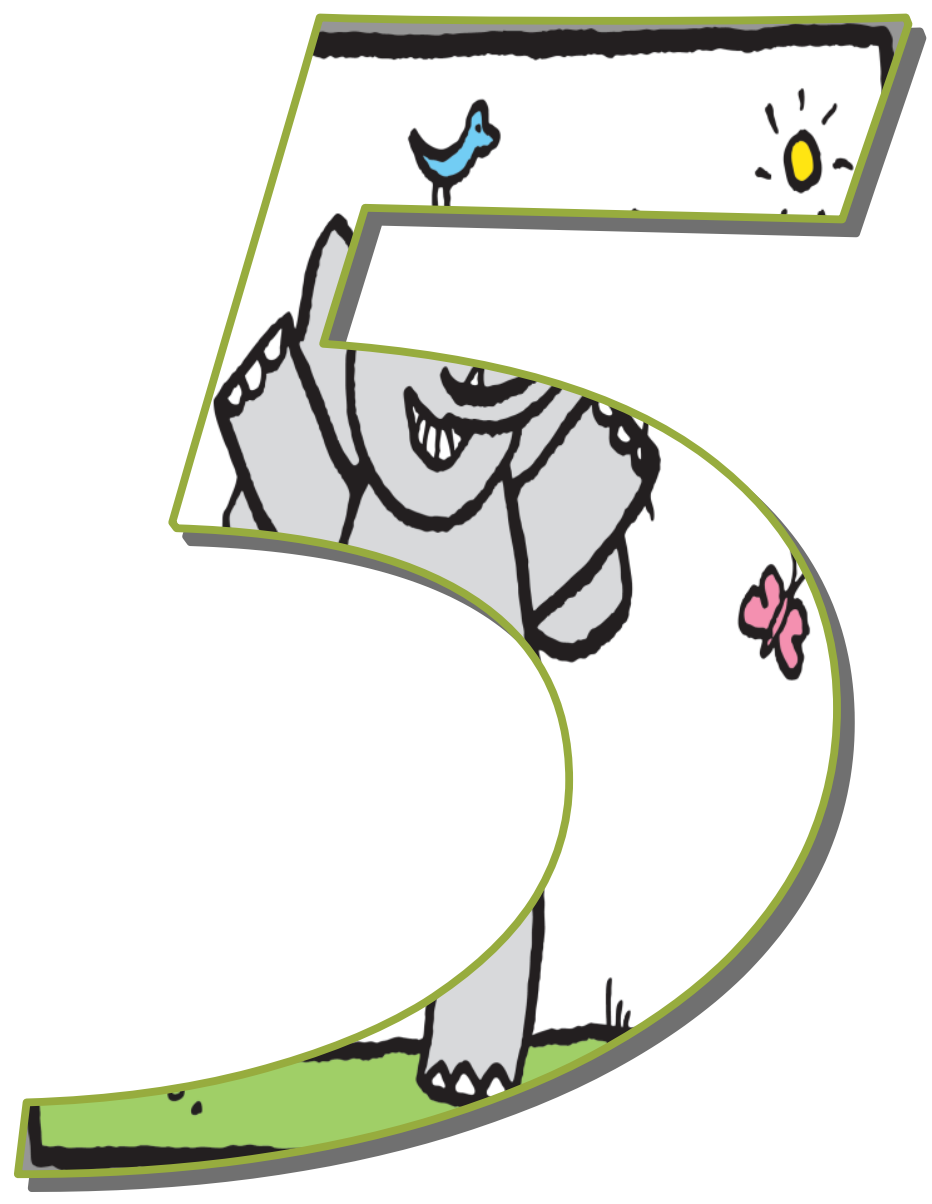

Early improvement in positive rather than negative emotion predicts remission from depression after pharmacotherapy 


\section{ABSTRACT}

Knowledge on mechanisms involved in early prediction of response to antidepressant medication may help optimize clinical decision making. Recent studies regarding response to pharmacotherapy implicate resilience-like mechanisms and involvement of positive, rather than negative emotions. The aim of the current study is to examine the contribution of early change in positive affect to the prediction of response to pharmacotherapy. Positive and negative emotions were measured at baseline and during the first week of pharmacotherapy, using experience sampling techniques. The association between early change in positive and negative emotions and severity of depressive symptoms at week six was examined in a sample of 49 depressed patients. The added benefits of measuring early change in positive emotions compared to early Hamilton Depression Rating Scale (HDRS) change alone were evaluated through model comparisons. Early improvement in positive affect during the first week of treatment predicted the continuous HDRS score $(6=-$ $0.64, p<0.001)$, response (50\% reduction; $O R=4.32, p<0.01$ ), and remission (HDRS $\leq 7 ; O R=9.29, p<0.001$ ) at week six with moderate to large effect sizes. Effects of early change in negative emotions were only half as large and disappeared when evaluated simultaneously with early change in positive emotions. When early change in positive emotions was added to the models including early HDRS change only, all three models improved significantly. In conclusion, early change in positive rather than negative emotions best predicted response to treatment, supporting the notion that antidepressants activate resilience-like mechanisms. Moreover, monitoring of positive emotions in early stages of treatment may improve clinical decision making.

Keywords: Affect; Depression; Treatment response; Experience sampling method; Early improvement; Prognosis

\section{Published as:}

Geschwind, N., Nicolson, N. A., Peeters, F., van Os, J., Barge-Schaapveld, D., \& Wichers, M. (2011). Early improvement in positive rather than negative emotion predicts remission from depression after pharmacotherapy. European Neuropsychopharmacology, 21(3), 241-247. doi: 10.1016/j.euroneuro.2010.11.004 
The efficiency of depression treatment can be considerably improved if current trial and error strategies are enriched with accurate outcome predictors at an early stage of treatment. In contrast to previous hypotheses on delayed onset of treatment response (Quitkin, Rabkin, Ross, \& Stewart, 1984), evidence suggests that about $60 \%$ of the improvement occurring on active antidepressant and placebo takes place during the first two weeks of treatment (Posternak \& Zimmerman, 2005). Several randomized or naturalistic studies as well as metaanalyses have shown that early improvement after one or two weeks of treatment strongly predicts later treatment outcome (e.g., Henkel, et al., 2009; Stassen, Angst, Hell, Schayfetter, \& Szegedi, 2007; Szegedi, et al., 2009; Tadic, et al., 2010). However, all these studies on early improvement assessed symptom severity with the Hamilton Depression Rating Scale (HDRS; Hamilton, 1960). The HDRS is a multidimensional instrument that covers only a selected number of clinical symptoms and neglects the assessment of positive emotions (Bagby, Ryder, Schuller, \& Marshall, 2004). Investigations that rely on the HDRS therefore provide little information on the nature of emotional changes driving long-term improvement in early responders. Better knowledge on the emotional mechanisms involved in early response may aid the development of better strategies for assessing early response to treatment, thereby optimizing clinical decision making and improving quality of life for patients suffering from depression.

Although studies show that antidepressant use affects both positive and negative emotion processing (e.g., Harmer, et al., 2010; Rawlings, Norbury, Cowen, \& Harmer, 2010), recent studies suggest that changes in positive rather than negative emotions may be important in predicting recovery from depression. First, recovery from depression was associated with increases in the ability to experience reward in daily life, rather than decreases in sensitivity to stress (Wichers, et al., 2009a). Second, a high ability to experience positive emotions in daily life was associated with both reduced risk of becoming depressed in individuals with high genetic loading for depression and increased resilience against the development of affective symptoms (Geschwind, et al., 2010; Wichers, et al., 2007a; Wichers, et al., 2010). Third, Stassen and colleagues (2007) concluded that antidepressants appear to trigger and maintain a common, resilience-like mechanism that controls recovery from depression. Psychological resilience can be defined as the ability to bounce back from negative emotional experiences, and recent studies show that positive, but not negative emotions, predict psychological resilience (Cohn, et al., 2009). 
Based on the findings outlined above, we hypothesized that early change in positive affect, rather than early change in negative affect, would be an important predictor of response to antidepressant treatment. Furthermore, we expected that adding information on early change in positive affect to information on early change in HRSD would significantly improve early prediction models of recovery from depression. We tested this hypothesis in a pre-existing longitudinal dataset on depressed patients starting pharmacotherapy. Early change in positive and negative emotions was measured using the experience sampling method (ESM) at baseline and at the end of the first week of pharmacotherapy. ESM is a momentary assessment technique in which participants are prompted to report on current emotions at random moments during the day, and is ideally suited for investigating change in emotions in daily life (Csikszentmihalyi \& Larson, 1987).

\section{EXPERIMENTAL PROCEDURES}

\section{Sample}

Eighty-three patients with a DSM-IV diagnosis of current major depressive disorder (MDD) were recruited in eight primary care practices in the Netherlands. Data were collected in the period between 1995 and 1996. Inclusion criteria were age between 18 and 65 years and a score at study entry of $\geq 18$ on the 17-item Hamilton Depression Rating Scale (HDRS; Hamilton, 1960). Exclusion criteria included major medical disorders and current use of psychotropic medications, except for the occasional use of temazepam. All participants gave written informed consent. The study was approved by the standing medical ethics committee.

\section{Study design}

During an initial baseline week, participants received no treatment in any form and took part in the experience sampling procedure during 6 days. Those who had completed at least 30 valid experience sampling assessments (for rationale see Experience Sampling Method below) were randomly assigned to twice-daily, double-blind, six-week treatment with either a tricyclic antidepressant or placebo (imipramine: starting dose of $50 \mathrm{mg} /$ day, increased to $200 \mathrm{mg} /$ day over the first week of treatment; placebo: starting with one capsule per day, increased to 4 capsules over the first week of treatment). In case of intolerance, the dose could be decreased to either $100 \mathrm{mg} /$ day of imipramine or 2 placebo capsules per day. Participants then took part in a second period of experience sampling during the last three days of the first week of treatment. In addition, patient's general practitioners (GPS) administered the HDRS at screening, and at the end of 
baseline, week 1 , week 2 , week 4 and week 6 . The current report used data from baseline, week 1 and week 6 .

\section{Experience Sampling Method}

The Experience Sampling Method (ESM) is a momentary assessment method to assess participants in their daily living environment, providing repeated in-themoment assessments of affect in a prospective and ecologically valid manner (Csikszentmihalyi \& Larson, 1987). Participants received a digital wristwatch and a set of ESM self-assessment forms collated in a booklet for each day. The wristwatch was programmed to emit a signal ("beep") at an unpredictable moment in each of ten 90-minute time blocks between 7:30 and 22:30, on consecutive days, resulting in a maximum of 60 beeps per person for the baseline measurement and a maximum of 30 beeps for the last three days of the first week. After each beep, participants were asked to fill out the ESM selfassessment, collecting reports of current mood and context. All self-assessments were rated on 7-point Likert scales. Participants were instructed to complete their reports immediately after the beep, thus minimizing memory distortion, and to record the time at which they completed the form. Participants with less than one third of valid reports were excluded from the analysis, and all reports not completed within $15 \mathrm{~min}$ after the actual beep were considered invalid, as previous work (Delespaul, 1995) has shown that reports completed after this interval are less reliable and consequently less valid.

\section{Measurements}

Hamilton Depression Rating Scale (HDRS)

The 17-item HDRS (Hamilton, 1960) was administered by the treating GP. All participating GPs had completed a standardized training for the HDRS.

Positive Affect (PA) and Negative Affect (NA)

At each beep, several ESM mood adjectives were assessed on 7-point Likert scales ranging from 1 (not at all) to 7 (very), as described previously (Barge-Schaapveld \& Nicolson, 2002). The mood adjectives 'cheerful', 'satisfied, 'energetic', 'calm', 'enthusiastic', 'strong' and 'happy' loaded on the PA factor (Cronbach's alpha=0.89), whereas 'hostile', 'depressed', 'tense', 'insecure', 'lonely', 'anxious', 'guilty', 'harried' and 'irritable' loaded on the NA factor (Cronbach's alpha=0.85). Mean levels of PA and NA were computed for each participant at each study period (baseline or end of week one) separately. 


\section{Statistical Analysis}

\section{Definitions of early change}

Early changes in HDRS, NA and PA were computed as the difference between the measure at baseline and at the end of week one, such that positive values reflect improvement (week one minus baseline for HDRS and NA change, baseline minus week one for PA change).

\section{Definitions of response and remission}

In agreement with commonly used definitions (e.g., Ballesteros, et al., 2007; Henkel, et al., 2009), response was defined as a reduction of $50 \%$ or more in HDRS score from baseline to week 6 , and remission as a score of 7 or less on the HDRS at week $6 .^{3}$

\subsubsection{Statistical tests}

Contributions of early change in PA and NA to improvement by week 6 were examined separately, using three different definitions of improvement: the continuous HDRS score at week 6, response, and remission. Simple linear regression with covariates was used to analyze the relationship of early change in PA and NA, respectively, with the continuous HDRS score at week 6, while correcting for baseline HDRS score. Logistic regression was used for the dichotomous outcomes response and remission. Because of our specific hypotheses on the importance of PA, further analyses focussed on early change in PA. Analyses explored to what extent early improvement in PA (compared to early deterioration or no change in PA) predicted improvement at week 6. Likelihood ratio tests were used to test whether early change in PA provided additional information over and above early HDRS change.

Imipramine and placebo groups were analyzed together, because mechanisms and trajectories of recovery have been found to be similar for antidepressant medication and placebo (Papakostas, Perlis, Scalia, Petersen, \& Fava, 2006; Posternak \& Zimmerman, 2005; Stassen, et al., 2007). Analyses were, however, corrected for group status (imipramine vs. placebo) and repeated in each group separately to verify the assumption that early change in PA predicted recovery from depression similarly in the placebo and TCA group.

\footnotetext{
${ }^{3}$ We chose to focus the analysis on outcome measures at week 6 because this is a clinically relevant time frame in which one can expect major changes in depressive symptoms. However, all analyses yielded similar results when repeated using definitions of stable response ( $50 \%$ reduction at both week 4 and week 6) and stable remission ( $\leq 7$ at week 4 and week 6 ), or when a growth model was used instead of linear regression analyses.
} 
Because average levels of affect at baseline influence the extent of possible change in PA or NA (i.e., ceiling or floor effects), all analyses were corrected for participant's average PA or NA at baseline. All variables were standardized, except for the outcome variables remission and response in the logistic regression. Standardized regression coefficients $\beta$ are reported for the linear regressions, and Odd's Ratios (OR) for the logistic regressions. Analyses were performed in Stata 11 (StataCorp, 2009).

\section{RESULTS}

\section{Participant characteristics}

Of the 83 recruited patients, 9 were excluded on the basis of the described inclusion and exclusion criteria. Additional 11 patients completed fewer than 30 valid ESM reports at baseline, which was the pre-set criterion for study continuation. Forty-nine of the remaining 63 participants $(78 \%)$ completed the first 6 weeks of treatment. Seventeen of the 49 participants received imipramine, and 22 placebo. Analyses were based solely on data of participants who completed treatment. Dropouts showed a trend towards higher PA at baseline, compared to completers $(\beta=0.31, p=0.09)$. There were no other significant baseline differences between dropouts and completers, and dropout was not associated with treatment group (imipramine vs. placebo).

Participants ranged in age from 25 to 59 years (mean $=42.5, \mathrm{SD}=9.2$ ). The majority were female $(73 \%)$ and married $(69 \%)$. Most had regular jobs $(47 \%)$ or were housewives (27\%). Average HDRS score ranged from 19 to 30 at baseline and from 0 to 24 at week 6 . Sixteen out of 49 participants were in remission in week 6 , and 30 were classified as responders. Means and standard deviations of PA, NA, and HDRS scores by study period are presented in Table 1 . Only 10 out of 49 participants ( 5 in the imipramine group, and 5 in the placebo group) experienced improvement of PA in the first week (defined as PA week $1>$ PA baseline), with no change or worsening in the others. Participants with early improvement of PA did not differ from the other participants in terms of sociodemographic characteristics and severity of depression (all $p>.20$ ). Figure 1 displays average changes in PA, NA, and HDRS during the first week of treatment, overall and stratified by response and remission status at week 6 . 
Table 1

Means and Standard Deviations (in brackets) of positive affect (PA), negative affect (NA) and Hamilton Depression Rating Scale (HDRS) scores in the overall sample, and stratified by response and remission status.

\begin{tabular}{lccccc}
\hline & Overall & \multicolumn{2}{c}{ Response } & \multicolumn{2}{c}{ Remission } \\
& & Yes & No & Yes & No \\
& $\mathrm{n}=49$ & $\mathrm{n}=30$ & $\mathrm{n}=19$ & $\mathrm{n}=16$ & $\mathrm{n}=33$ \\
\hline PA baseline & $3.5(1.4)$ & $3.5(1.6)$ & $3.5(1.2)$ & $3.3(1.6)$ & $3.6(1.4)$ \\
PA week 1 & $3.0(1.4)$ & $3.3(1.5)$ & $2.4(1.0)$ & $3.6(1.4)$ & $2.6(1.2)$ \\
NA baseline & $3.9(1.3)$ & $3.5(1.3)$ & $4.4(0.9)$ & $3.5(1.4)$ & $4.1(1.1)$ \\
NA week 1 & $3.3(1.5)$ & $2.8(1.3)$ & $4.1(1.4)$ & $2.5(1.0)$ & $3.7(1.5)$ \\
HDRS baseline & $24.0(3.2)$ & $24.0(3.6)$ & $24.1(3.1)$ & $23.3(3.8)$ & $24.4(2.9)$ \\
HDRS week 1 & $21.5(4.7)$ & $20.4(5.1)$ & $23.3(3.5)$ & $19(5.3)$ & $22.7(3.9)$ \\
HDRS week 6 & $10.9(6.5)$ & $6.5(3.4)$ & $17.8(3.5)$ & $4.0(2.5)$ & $14.0(5.0)$ \\
\hline
\end{tabular}

\section{Association of Early Change in PA and NA with recovery from depression}

Early change in PA during the first week of treatment significantly predicted continuous HDRS scores as well as response and remission at week six. As shown in Table 2, effect sizes of early change in PA were moderate to large. Expressed in unstandardized values, an increase of one point (on the 7-point Likert scale) in PA from baseline to week one corresponded to a 4-point decrease on the HDRS by week six. Experiencing early improvement of PA, compared to no change or early worsening of PA, was associated with a 9-point decrease on the HDRS in week six ( $\beta=-0.54, p<0.001$ ) and a 34 times higher probability of achieving remission (OR $=33.90, p<0.01)$. All 10 participants with early improvement of PA met the response criterion at week six. Effect sizes of early change in PA remained moderate to large when the analyses were repeated separately for the placebo group (continuous: $\beta=-0.54, p<0.01$, response: $O R=3.89, p<0.05$, remission: $O R=6.52, p<0.05$ ) and the imipramine group (continuous: $\beta=-0.63, p<0.01$, response: $O R=5.38, p=0.07$, remission: $O R=20.41, p<0.05)$. 
no

no
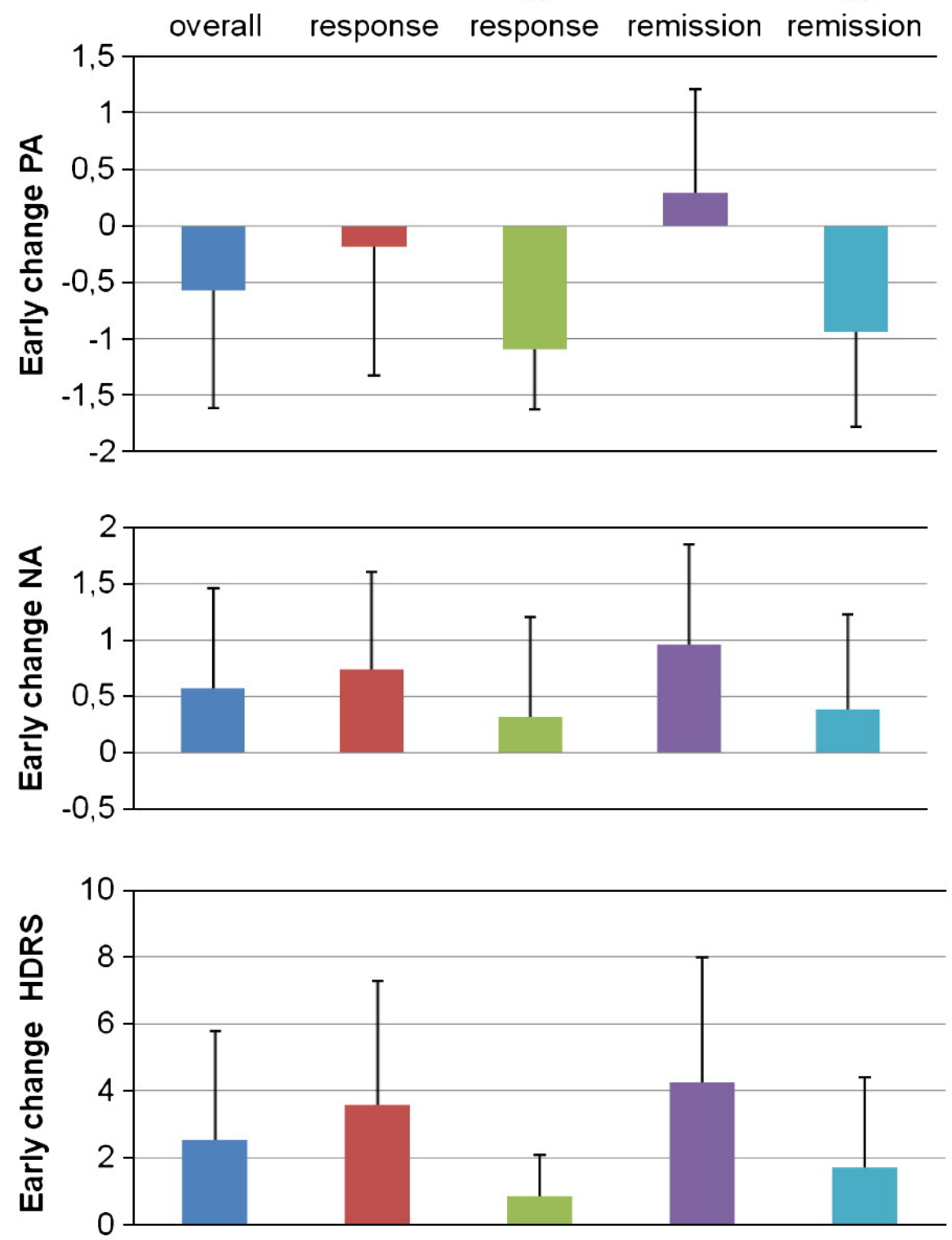

Figure 1. Average change in positive affect (PA), negative affect (NA) and Hamilton Depression Rating Scale (HDRS) after one week of treatment. 
Table 2

Standardized effect sizes and significance levels of early change in positive affect (PA) or negative affect (NA) on Hamilton Depression Rating Scale (HDRS) score, response and remission at week 6 (first column).

\begin{tabular}{|c|c|c|c|c|}
\hline & effect size & effect size & effect size & effect size \\
\hline & $\begin{array}{c}\text { Early } \\
\text { change PA }\end{array}$ & Baseline PA & Group & $\begin{array}{c}\text { Baseline } \\
\text { HDRS }\end{array}$ \\
\hline HDRS score $(\beta)$ & $-0.64 * * *$ & $-0.34 *$ & $-0.35 * *$ & 0.05 \\
\hline Response (OR) & $4.32 * *$ & 2.25 & 1.56 & - \\
\hline \multirow[t]{2}{*}{ Remission (OR) } & $9.29 * * *$ & 2.91 & $3.12 *$ & - \\
\hline & $\begin{array}{c}\text { Early } \\
\text { change NA }\end{array}$ & $\begin{array}{c}\text { Baseline } \\
\text { NA }\end{array}$ & Group & $\begin{array}{c}\text { Baseline } \\
\text { HDRS }\end{array}$ \\
\hline HDRS score $(\beta)$ & $-0.32 *$ & 0.32 & -0.24 & 0.07 \\
\hline Response (OR) & 1.86 & $0.42 *$ & 1.15 & - \\
\hline Remission (OR) & $3.58 *$ & 0.41 & 1.74 & - \\
\hline
\end{tabular}

Although early change in NA also was a significant predictor for the continuous HDRS sore and remission status (but not for response) at week 6 , effect sizes were only about half the size of those for early change in PA (see Figure 2). When early change in PA and early change in NA were entered simultaneously to check for the independence of their contributions, effect sizes of early change in PA remained significant and similar in size (continuous: $\beta=-$ 0.57, $p<0.001$, response: $O R=4.00, p<0.05$, remission: $O R=6.94, p<0.01$ ), while the effects of early change in NA decreased and were no longer statistically significant (continuous: $\beta=-0.12, p>.3$, response: $O R=1.32, p>.4$, remission: $O R$ $=1.62, p>.4)$, indicating that the predictive value of NA is reducible to that of PA.

Improvement of early prediction models through addition of early change in PA Early change in HDRS predicted significant improvement on all three outcome measures (continuous: $\beta=-0.48, p<0.001$, response: $O R=4.08, p<0.05$, remission: $O R=2.36, p<0.05)$. When early change in PA was added to the model including early change in HDRS, all three models improved significantly (continuous: $\operatorname{chi2}(2)=12.90, p<0.01$; response: $\operatorname{chi2}(2)=6.24, p<0.05$; remission chi2 $(2)=14.72, p<0.001)$, with the proportion of explained variance increasing from $30 \%$ to $44 \%$, from $18 \%$ to $28 \%$, and from $16 \%$ to $40 \%$, respectively. 

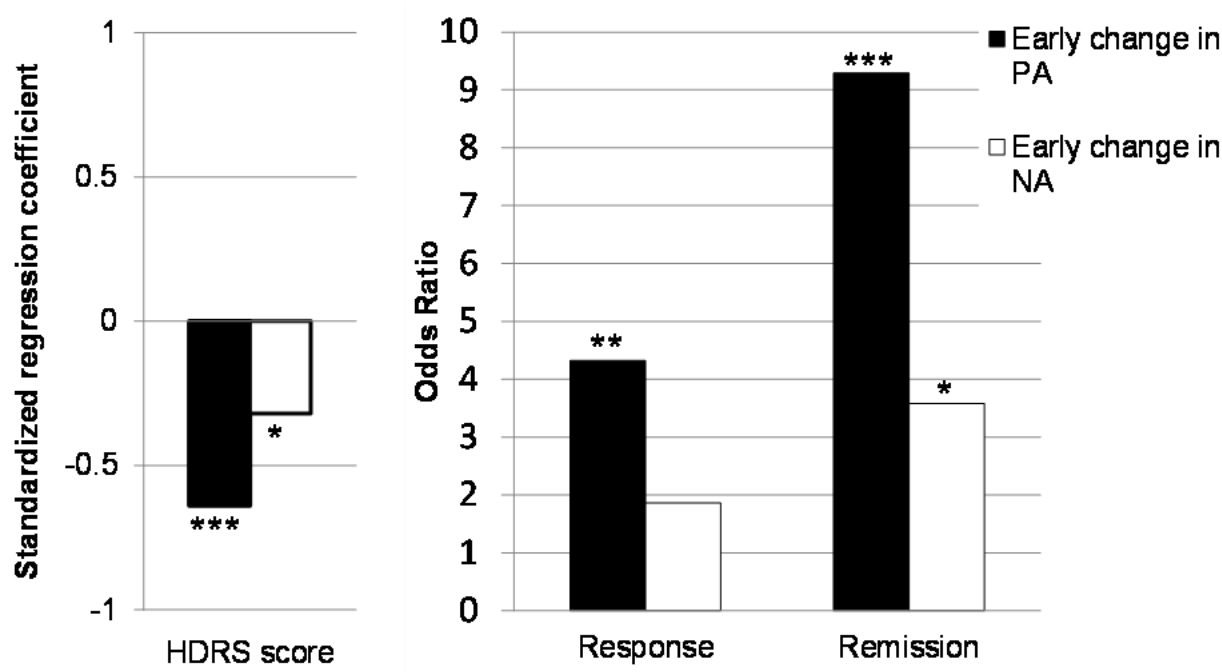

Figure 2. Graph with standardized effect sizes of early change in positive affect (PA) and negative affect (NA) predicting Hamilton Depression Rating Scale (HDRS) score, response (50\% HDRS reduction), and remission (HDRS $\leq 7$ ) at week 6.

\section{DISCUSSION}

Early change in Positive Affect (PA), compared to Negative Affect (NA), best predicted response to treatment. Early change in PA during the first week of treatment was associated with improvement on the Hamilton Depression Rating Scale (HDRS), response ( $50 \%$ symptom reduction), and remission (HDRS $\leq 7$ ) at week six. Effect sizes were moderate to large. Early change in NA was not consistently associated with response to treatment, and effect sizes were much smaller than those of early change in PA. When both predictors were entered into the regression model simultaneously, the effects of NA change diminished even further, whereas effect sizes of early change in PA remained similar. Compared to no change or early worsening of PA, experiencing early improvement of PA was associated with a 34 times higher chance of achieving remission, and all 10 participants with early improvement of PA achieved response at week six. Early PA improvement did not depend on treatment group (imipramine or placebo). Because participants with early improvement of PA did not differ from the other participants in terms of socio-demographic characteristics and severity of depression, differences in outcome can likely be attributed to changes in PA, rather than to third variables associated with PA. Finally, adding early change in 
positive emotions to standard early prediction models significantly improved accuracy of prediction at week six for all three outcome measures.

In order to speed up recovery from depression and provide adequate treatment, it is useful to identify non-responders as early and as accurately as possible. Early change in PA provided vital information for prediction of response over and above early HDRS change, which is still used as gold standard for the assessment of depression, despite psychometrical and conceptual flaws (Bagby, et al., 2004). The HDRS unfortunately does not measure PA at all. It contains only one item on "Work and Interest", in which patients' capacity to work is assessed simultaneously with their hospitalization status and levels of interest in activities (Hamilton, 1960). We argue that assessment of PA may aid early identification of (non)response and that assessment of PA should be included when monitoring early treatment response. More research is needed before determining clinically informative cut-off points which eventually could be used to aid clinical decision making involved in the treatment of depression. Another unanswered question is which time frame is best for assessing early changes in PA. Early improvement in this study was very successful in identifying responders to treatment (i.e., all early PA improvers met the response criterion at week six). However, the low rate of early PA improvement (compared to reported rates of $65 \%$ of early HDRS improvement; Szegedi, et al., 2009) may mean that measuring PA change after two weeks could be more successful in identifying non-response and need for adjustment of treatment.

Because studies indicate that PA is associated with resilience and well-being more strongly than NA (Cohn, et al., 2009; Fredrickson, Tugade, Waugh, \& Larkin, 2003), our findings support Stassen et al.'s (2007) claim that antidepressant treatment may activate resilience-like mechanisms. Earlier studies found that positive emotions were associated with attenuated expression of genetic risk for depression and increased resilience after adversity (Geschwind, et al., 2010; Wichers, et al., 2008b; Wichers, et al., 2007a; Wichers, et al., 2010). Furthermore, in an experimental setup, depressed participants differed from healthy controls only regarding emotional response to anticipated reward but not punishment (McFarland \& Klein, 2009). The current study adds to the accumulating evidence on the importance of positive emotions in depression by showing that, after as early as one week of treatment, the PA system reacts sensitively to treatment and is closely linked to later response. Whether PA is part of the resilience response or rather facilitates action of other resilience-related mechanisms remains unclear and may be investigated in future research. 
Several large studies as well as meta-analyses demonstrate that trajectories of recovery under active antidepressants - once triggered - follow a pattern similar to that of placebo, despite marked pharmacologic differences of the triggers (Posternak \& Zimmerman, 2005; Stassen, et al., 2007; Szegedi, et al., 2009; Tadic, et al., 2010). Also in the current study, early change in PA predicted response to treatment in both the imipramine and the placebo group, implying overlapping mechanisms of recovery from depression in both groups. As Stassen and colleagues (2007) argue, this may mean that depressed patients have a biological resilience-like predisposition that controls the time course of recovery and that can be triggered, modified or suppressed. Our study suggests that drugs - or psychotherapies - actively targeting the PA or reward system may be particularly effective in activating resilience mechanisms leading to recovery from depression. Drugs that modulate catecholaminergic activity may increase PA more than other types of drugs (Depue, Luciana, Arbisi, Collins, \& Leon, 1994; Nutt, et al., 2007) because they tend to elevate dopamine activity in the prefrontal cortex (Karson, et al., 1983). Tomarken et al. (2004), for example, reported that the catecholaminergic action of bupropion SR produced more robust effects on anhedonia than placebo (although see Dichter, Tomarken, Freid, Addington, \& Shelton, 2005). It is possible that drugs that facilitate early changes in PA are more efficient in triggering recovery processes, even though these differences in PA may disappear as time goes by and patients recover from depression.

In conclusion, the involvement of positive emotions in early response to antidepressant treatment supports the activation of resilience-like mechanisms in recovery from depression. Moreover, monitoring of positive emotions in early stages of treatment may improve clinical decision making.

Limitations of the study include that evaluation of response and remission took place in week 6 . Our results need to be replicated in studies with longer follow-up periods and larger samples. Another limitation is that follow-up measurements of dropouts were unavailable, possibly distorting the results. However, analyses are likely to underestimate rather than overestimate the effects of early PA improvement, given that 10 of 11 dropouts were participants with non-improvement of PA. Finally, the relatively small sample size only allowed for investigations of mechanisms of change common to placebo and active antidepressant medication. However, several large studies suggest that early improvement mechanisms appear to be similar in placebo and active drugs (e.g., Stassen, et al., 2007; Szegedi, et al., 2009), and early change in positive affect was an important predictor of improvement also when both groups (placebo and imipramine) were examined separately. 


\section{Acknowledgements}

We thank P. Verburg-Noteborn, C. van der Voort-Duindam, G. Driesen and F. van Goethem for help with data collection and processing. M. de Vries and P. Delespaul provided advice on various aspects of the study. We are indebted to M. Bemelmans, R. Brouwers, G. Costongs, A. Schlösser, J. Theunissen, P. van Aubel, D. van der Voort, and M. Weijts for subject recruitment and clinical assessments. The original study was supported by an unrestricted research grant from Solvay Pharmaceuticals, Weesp, the Netherlands. M.C. Wichers was supported by the Dutch Organisation for Scientific Research (VENI grant nr 916.76.147). 


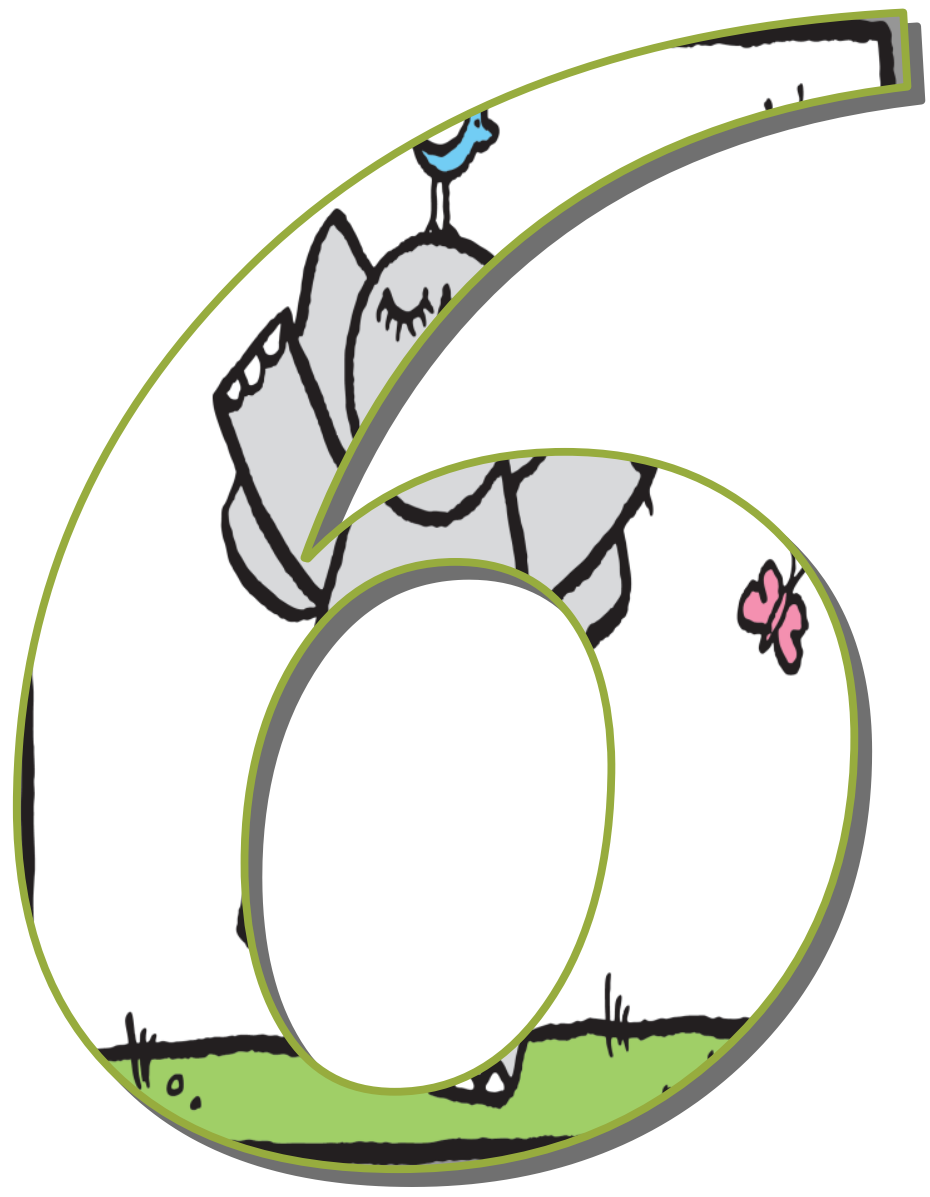

Meeting risk with resilience:

High daily-life reward experience preserves mental health 
Objective: To examine prospectively whether high reward experience (the ability to generate positive affect boosts from pleasurable daily events) protects against affective symptoms and whether environmental or genetic risk factors moderate protective effects.

Method: At baseline, 498 female twins participated in an experience sampling study measuring reward experience in daily life. They also completed questionnaires on childhood adversity and recent stressful life events (SLE). Affective symptoms were measured at baseline and at four follow-ups using SCL90 anxiety and depression subscales. Co-twin affective symptoms were used as indicators of genetic risk.

Results: Baseline reward experience did not predict follow-up affective symptoms, regardless of level of genetic risk. However, high reward experience was associated with reduced future affective symptoms after previous exposure to childhood adversity or recent SLE.

Conclusions: High daily-life reward experience increases resilience after environmental adversity; modification of reward experience may constitute a novel area of therapeutic intervention.

Keywords: affective symptoms; resilience, psychological; risk factors; longitudinal studies; twin study

Published as:

Geschwind, N., Peeters, F., Jacobs, N., Delespaul, P., Derom, C., Thiery, E., ... Wichers, M. (2010). Meeting risk with resilience: High daily-life reward experience preserves mental health. Acta Psychiatrica Scandinavica, 122(2), 129-138. doi: 10.1111/j.1600-0447.2009.01525.x 


\section{Significant Outcomes:}

- The ability to generate positive affect boosts (reward experience) from pleasant daily-life events preserves mental health, but only in case of high childhood adversity or recent stressful life events.

- High daily-life reward experience may thus represent a mechanism of resilience in people at risk for depression and related disorders.

- Novel treatments for the prevention of affective disorders should focus on enhancing daily-life reward experience in people who experienced early or late environmental adversity. Discovering how to enhance reward experience remains a challenge for future research.

\section{Limitations:}

- The observations of the Experience Sampling data rely on participants' compliance.

- Childhood trauma and recent SLE were measured retrospectively.

- Because participants were female only, the results may not be generalizable to men.

\section{INTRODUCTION}

"The foolish man seeks happiness in the distance, the wise grows it under his feet" (James Oppenheim). This quote refers to the ability to enjoy the moment instead of living in the future. Some people are able to generate an abundance of positive emotions from pleasant everyday events like going to a birthday party, seeing an inspiring movie or eating tasty food. In other words, they have the ability to use pleasant events to boost their mood, at least temporarily, be it deliberate or non-deliberate. Does this ability make them more resilient in the long run? Here we examine prospectively whether the ability to generate boosts in positive affect (PA) in response to pleasurable daily-life events is associated with more favourable mental health outcomes.

In general, PA is a strong candidate for resilience against stress-related disorders, because it reduces stress-induced psychiatric and physiological symptoms (Danner, et al., 2001; Fredrickson, 2001; Fredrickson, Cohn, Coffey, Pek, \& Finkel, 2008; Tugade \& Fredrickson, 2004; Zautra, et al., 2005). In daily life, experiencing PA during moments of minor stress is associated with lowered sensitivity to stress. In other words, the usual increase in negative affect (in reaction to stressful events) is less pronounced when people experience PA (Wichers, et al., 2007a). Furthermore, Fredrickson and colleagues (2008) recently 
found that experiencing more PA predicted increased personal resources (like social support and purpose in life), which in turn predicted decreased affective symptoms. Similarly, a retrospective study showed that maintaining near-normal levels of positive emotions during the aftermath of the September 11 terrorist attacks was associated with less affective symptoms a couple of weeks later (Fredrickson, et al., 2003). These studies show that PA buffers the effects of stressful events.

Apart from that, PA also reduces the expression of genetic vulnerability for affective disorders. Individuals with genetic vulnerability for depression are more stress-sensitive than individuals without genetic vulnerability (Wichers, et al., 2007b). However, in situations of high PA, the difference in stress sensitivity between genetically vulnerable and non-vulnerable individuals is reduced (Wichers, et al., 2007a). A study using direct genetic information found similar results: Experiencing positive emotions neutralizes the difference in stress sensitivity caused by the BDNF Val ${ }^{66}$ Met genotype (Wichers, et al., 2008b).

Thus, the existing literature demonstrates that PA preserves, and possibly improves, mental health. Preserving mental health becomes particularly important in the presence of environmental or genetic risk. Childhood adversity (Afifi, Brownridge, Cox, \& Sareen, 2006; Kendler, et al., 2004) as well as recent stressful life events (SLE) (Hammen, 2005; Kessler, 1997; Van Praag, De Kloet, \& Van Os, 2004a) are important environmental risk factors, because they increase daily-life stress sensitivity as well as the risk for major depression and related disorders (Glaser, et al., 2006; Wichers, et al., 2009c).

Given the many benefits of PA, we expect that the ability to generate boosts in PA from pleasant daily-life situations (hereafter: high reward experience) will contribute to the preservation of mental health. We predict that high reward experience, measured at baseline, will predict low affective symptoms at followup, especially when someone is at risk for low mood (due to either environmental adversity like childhood adversity or recent SLE or genetic risk), see Figure 1 . We tested these hypotheses in a prospective twin study, using the experience sampling method (ESM). ESM is optimally equipped to test these hypotheses, because it measures people's experiences in their own daily-life context, not only prospectively but also repetitively, catching variability in the flow of daily life. This type of measurement is necessary to reliably capture such a dynamic concept as daily-life reward experience. 


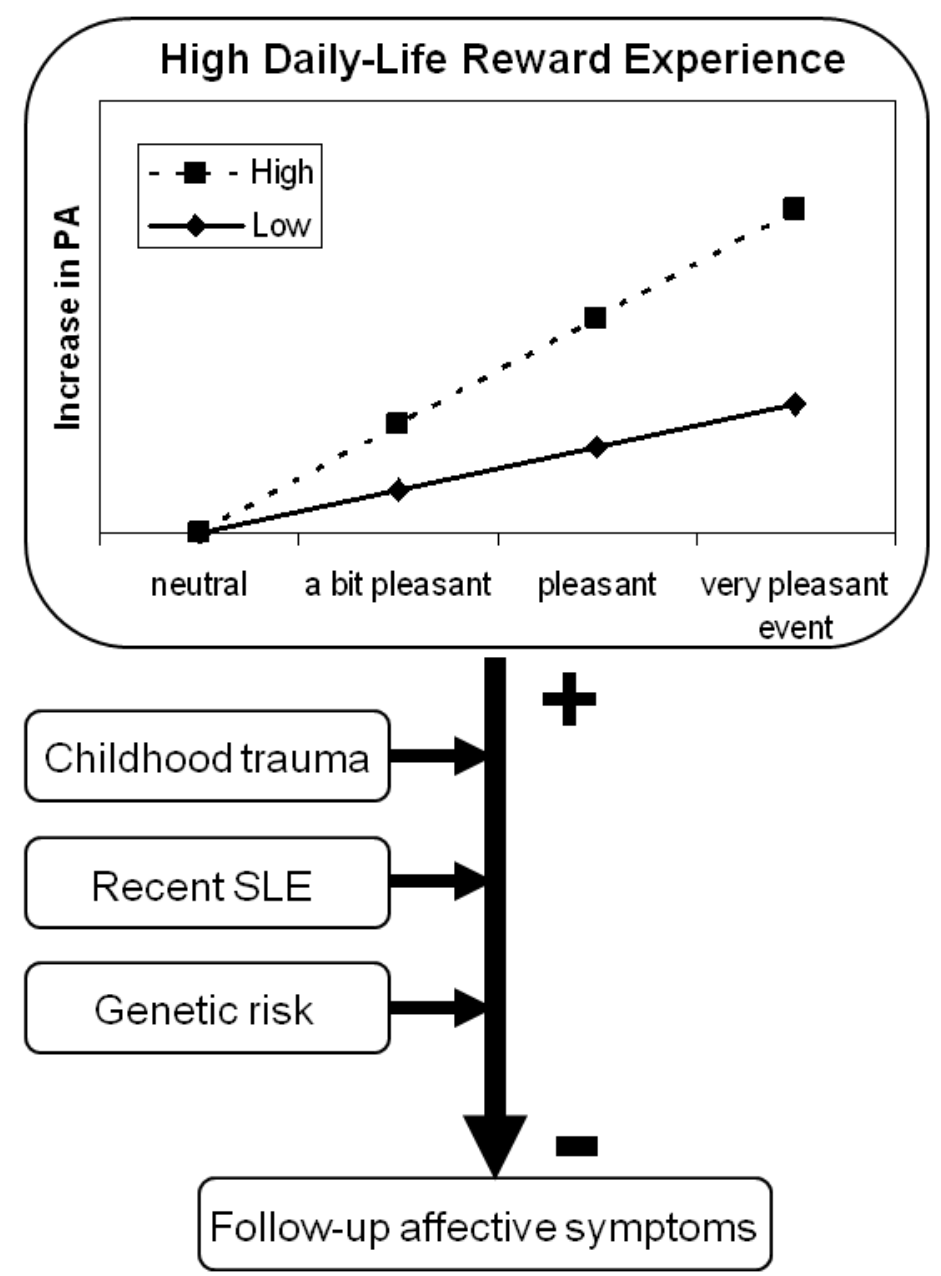

Figure 1. Daily-Life Reward Experience and expected relations with follow-up affective symptoms. People with high reward experience are able to generate stronger positive affect (PA) boosts in response to pleasant events than people with low reward experience. Higher daily-life reward experience is hypothesized to be associated with less follow-up affective symptoms, especially in people at risk for affective symptoms, i.e., those with higher levels of childhood trauma, recent stressful life events (SLE), and genetic risk.

\section{Aims of the study}

The aims of our study are to examine prospectively whether high reward experience (the ability to generate positive affect boosts from pleasant events) contributes to resilience against affective disorders, and how this protective effect is moderated by environmental and genetic risk factors. 


\section{MATERIAL AND METHODS}

\section{Sample}

The study sample consisted of 621 female participants (age: 18 to 61) from Flanders (Belgium). The participants were general population twin pairs and 46 of their non-twin sisters. Two-hundred and eighteen of these twin pairs were recruited from the East-Flanders Prospective Twin Survey (EFPTS), which has prospectively recorded all multiple births in the province of East-Flanders since 1964 (Derom, et al., 2006; Loos, et al., 1998). Twin zygosity was determined at birth for EFPTS participants. For the other participants, zygosity was determined through a sequential analysis based on sex, fetal membranes, blood groups, DNA fingerprints, and interviews (Jacobs, et al., 2006). The study was approved by the Ethics Committee of Maastricht University, and all participants provided written informed consent.

\section{Experience Sampling Method (ESM)}

ESM is a structured diary technique to assess participants in their daily living environment. ESM has been validated for the use of studying the immediate effects of daily-life situations on mood (Csikszentmihalyi \& Larson, 1987; De Vries, 1992; Delespaul, 1995; Lataster, et al., 2009). Participants received a digital wristwatch and a set of ESM self-assessment forms collated in a booklet for each day. The wristwatch was programmed to emit a signal ("beep") at an unpredictable moment in each of ten 90-minute time blocks between 7:30 and 22:30, on five consecutive days, resulting in a maximum of 50 beeps per person. After each beep, participants were asked to stop their activity and fill out the ESM self-assessment forms, with regard to thoughts, mood, current context (activity, persons present, and location) and appraisals of current situation and, finally, the most important event since the last beep. All self-assessments were rated on 7point Likert scales.

Trained research assistants with ample experience in momentary assessment techniques explained the ESM procedure to the participants during an initial briefing session, and participants completed an ESM practice form to confirm that they understood the 7-point Likert scale. Participants could call a telephone number in case they had questions or problems during the ESM sampling period. Participants were told to complete their reports immediately after the beep, thus minimizing memory distortion, and to record the time at which they completed the form. In order to know whether the participants had completed the form within $15 \mathrm{~min}$ of the beep, the time at which participants indicated they completed the report was compared to the actual time of the 
beep. All reports not filled in within $15 \mathrm{~min}$ after the beep were excluded from the analysis, since previous work (Delespaul, 1995) has shown that reports completed after this interval are less reliable and consequently less valid. In addition, previous work has shown that participants who have valid reports for at least onethird of all measurements can be included since their missing data does not distort the results, whereas measures of individuals with fewer than $30 \%$ of completed reports are less reliable (Delespaul, 1995). Therefore, participants with fewer than 17 valid reports (out of 50) were excluded from the analysis.

\section{Design}

Participants were assessed at baseline (ESM, childhood adversity, recent SLE, and affective symptoms) and at four follow-up time points (affective symptoms). The average number of days between baseline and first follow-up was 132, between first and second follow-up 91, between second and third follow-up 116 and between third and fourth follow-up 91.

\section{Measurements}

Affective symptoms

Participants completed the Symptom Checklist (SCL-90R) (Bech, 2004; Derogatis, 1994) at baseline and at four follow-up assessments. The SCL-90R is a comprehensive self-report symptom inventory, multidimensional in nature, and oriented to screen for a broad range of psychological problems and psychopathology in community respondents. It contains 90 items, scored on a five-point severity scale, measuring 9 primary symptom dimensions named 'Somatization', 'Obsessive-Compulsive', 'Interpersonal Sensitivity', 'Depression', 'Anxiety', 'Hostility', 'Phobic Anxiety', 'Paranoid Ideation' and 'Psychoticism'.

Per participant, SCL-90R depression and anxiety scales were averaged for each measurement occasion, resulting in a combined score reflecting affective symptoms.

\section{Childhood adversity}

Childhood adversity was measured using the shortened version (Bernstein, et al., 1997) of the 70-item Childhood Trauma Questionnaire (Arntz \& Wessel, 1996; Bernstein, et al., 1994). At the request of the Twin Registry, the most explicit items concerning sexual and physical abuse were omitted; less explicit items were retained. The questionnaire thus consisted of 21 items with statements concerning early life experiences, such as "I was abused", "There was not enough food" and "I was neglected". Items were scored on a scale of 1 (never true) to 5 
(very often true). Cronbach's alpha for this 21 -item questionnaire was 0.93 . The sum score of all items was used as a continuous measure of childhood adversity.

\section{Recent SLE}

Our inventory of recent SLE was based on the event list of the Interview for Recent Life Events (Paykel, 1997). Participants reported whether any of 61 events happened in the past 6 months and indicated how unpleasant the experience was to them (from $1=$ very pleasant to $5=$ very unpleasant). SLE were divided into 10 categories: work, education, finance, health, bereavement, migration, courtship and cohabitation, legal, family and social relationships, and marital relationships, all representing datable occurrences involving changes in the external social environment. Only SLE rated as unpleasant (i.e., with a score of 4, "unpleasant," or a score of 5, "very unpleasant") were included in the analysis. The number of such unpleasant events in the past 6 months was used as a continuous measure of recent SLE (Jacobs, et al., 2006).

\section{Genetic risk for affective symptoms}

Genetic risk for affective symptoms was assessed indirectly using information on the affective health of the co-twin. The continuous variable "co-twin affective symptoms" was constructed, representing baseline affective symptoms of the participant's co-twin. To obtain an indicator of genetic risk for depression, this variable was paired with information about twin zygosity (mono- or dizygotic).

Daily-life events, affect and reward experience

Measures of daily-life events and affect were extracted from the Experience Sampling Method (ESM) framework, Participants rated their current mood on 7point Likert scales ranging from 0 (not at all) to 7 (very much). The mean of the positive affect items (I feel 'cheerful', 'content', 'energetic', and 'enthusiastic') formed the Positive Affect (PA) scale (Cronbach's alpha $=0.86$ over the participant mean). Negative affect (NA) was assessed with six mood adjectives (I feel 'insecure', 'lonely', 'anxious', 'low', 'guilty', and 'suspicious') and had a Cronbach's alpha $=0.76$ over the participant mean. After rating their affect, participants reported the most important event that happened between the current and the previous beep and rated that event on a 7-point bipolar scale $(-3=$ very unpleasant, $0=$ neutral, $3=$ very pleasant). Reward experience was conceptualised as the effect of pleasant daily-life events (i.e., only those events rated as 0 (neutral) to 3 (very pleasant)) on PA (Wichers, et al., 2008a). For each participant reporting at least four pleasant events, a reward experience variable was constructed by regressing beep-moment PA on the pleasantness score of the most recently reported daily-life event (Wichers, et al., 2008a). This variable thus 
reflects the extent to which the pleasantness of daily-life events translates into increases in PA, see Figure 1. Some people, for example, experience just a small increase in PA after an event they rated as very pleasant, say a birthday party. Others experience a much larger increase in PA, suggesting that they can use the birthday party more effectively to generate positive emotions. Stress-sensitivity was conceptualised likewise as negative affect reactivity to unpleasant daily-life events, see Wichers and colleagues (2007b).

\section{Analysis}

This study consisted of 5 measurement occasions. Most of the participants were twins. This means that the data were clustered: the five measurement occasions were clustered within participants, who were clustered in twin pairs. Clustering induces correlation in the data: measurements within participants are more alike than measurements between participants, and measurements within twin pairs are more alike than measurements between twin pairs. Appropriate analysis techniques are necessary to handle the correlations induced by the clustered data (Snijders \& Bosker, 1999). We used the STATA XTMIXED multilevel linear regression command.

All analyses were corrected for baseline SCL-90R affective symptoms to ensure that associations between follow-up affective symptoms and baseline variables reflected effects on change in affective symptoms. All variables included in the analyses were standardized, yielding standardized effect sizes.

To examine our hypotheses, we ran the following regression analyses: The main effect of reward experience on follow-up affective symptoms was examined by regressing affective symptoms on reward experience. Regarding the hypothesis that risk factors moderate the effect of reward experience on affective symptoms, follow-up affective symptoms were regressed on the interaction between the risk factor and reward experience. This was done separately for each of the three risk factors (childhood adversity, recent SLE, and genetic risk). For childhood adversity and recent SLE, this resulted in regressions with a 2-way interaction (childhood adversity $\mathrm{x}$ reward experience / recent SLE $\mathrm{x}$ reward experience). Significant interactions were followed up by dose-response analyses. Dose-response relationships were examined by dividing the distribution of values for childhood adversity and recent SLEs by their tertiles using the STATA XTILE command. The STATA LINCOM command was used subsequently to calculate effect sizes of the differences between the low risk group and the medium or high risk group, respectively. For genetic risk, the regression contained a three-way interaction (co-twin affective symptoms $x$ zygosity $x$ reward experience). The STATA LINCOM 
command was used to calculate stratified effect sizes for mono- and dizygotic twins.

When risk factors were significantly associated with each other, the independence of their effects was checked by simultaneously entering their interactions (with reward experience) into the regression model.

Finally, the analyses were repeated in order to correct for a number of potential confounders. First, it is known that stress sensitivity influences risk for affective symptoms (2007b). To ensure that possible protective effects of high reward experience were not an artefact of low stress sensitivity, all analyses were repeated whilst correcting for stress sensitivity. Second, all analyses were repeated excluding the 20 participants who were depressed at baseline, to verify that the retrospective measures of childhood adversity and recent SLE were not contaminated by current depression status. Third, all analyses were re-run whilst additionally correcting for the number of positive events experienced by each participant and for the average PA per participant.

\section{RESULTS}

\section{Participant characteristics}

Of the total sample of 621 participants, 610 participated in the ESM procedure. Thirty-one participants were excluded because they had fewer than 17 valid ESM self-reports. Another 81 participants were excluded due to missing data on either (i) affective symptoms at all follow-ups, (ii) baseline reward experience or (iii) baseline affective symptoms. This resulted in a dataset of 498 participants that were part of 246 different twin pairs. Of the 498 participants, 286 were monozygotic twins, 170 were dizygotic twins, two were twins of unknown zygosity, and 40 were non-twin sisters of twins. For the analysis on genetic risk, non-twin sisters and twin-pairs of unknown zygosity were excluded. Of the remaining twins, 13 had missing data on co-twin affective symptoms, leaving 443 participants (280 monozygotic and 163 dizygotic twins) in the analyses involving genetic risk. Five of the 498 participants had missing data on childhood adversity, leaving 493 participants in the analyses involving childhood adversity. Mean age of the 498 participants was 28 years (SD: 7.9 years, range: 18-61). Sixty-five percent had a college or university degree, $33 \%$ completed secondary education and $1 \%$ had primary education only. The majority was currently employed $(60 \%$ employed, $31 \%$ student, $2 \%$ unemployed, $3 \%$ homemaker and $1 \%$ sick leave). Ninety-seven percent participated in at least two, $91 \%$ in at least three, and $79 \%$ in all four follow-ups. 
Multilevel analyses showed no significant association between reward experience on the one hand and genetic risk $\left(\beta=-0.15, \chi^{2}(1)=2.84, p=0.11\right)$, childhood adversity $(\beta=0.08, p=0.09)$, and recent $\operatorname{SLE}\left(\beta=0.03, \chi^{2}(1)=0.50\right.$, $p=0.48)$ on the other. Childhood adversity was significantly associated with later experience of SLE $\left(\beta=0.21, \chi^{2}(1)=40.38, p=0.00\right)$. Therefore, the independence of their interaction terms was tested (see Independence of moderation later on). Other risk factors were not associated with each other. Mean score of affective symptoms was $1.47(\mathrm{SD}=0.50)$ at baseline and $1.42(\mathrm{SD}=0.48)$ at follow-up assessments. Mean score of childhood adversity was $1.64(\mathrm{SD}=0.55)$, and mean score of recent SLE was $2.1(S D=2.3)$.

\section{Associations between reward experience and future affective symptoms}

\section{Main effect}

There was no overall association between reward experience and later affective symptoms $\left(\beta=0.02, \chi^{2}(1)=0.49, p=0.49\right)$.

Moderation by childhood adversity

The two-way interaction between childhood adversity and reward experience in the model of affective symptoms was significant $\left(\beta=-0.07, \chi^{2}(1)=6.05, p=0.01\right)$ : After childhood adversity, high reward experience was associated with less future affective symptoms. A dose-response association was apparent in that the effect of reward experience on future affective symptoms became stronger with higher levels of childhood adversity (average compared to low childhood adversity: $\beta=$ $0.06, \chi^{2}(1)=0.85, p=0.36$; high compared to low childhood adversity: $\beta=-0.15$, $\left.\chi^{2}(1)=4.65, p=0.03\right)$. For effect sizes of baseline daily reward experience on follow-up affective symptoms, stratified by degree of childhood adversity, see Figure 2. Descriptive information on the average levels of affective symptoms for high and low levels of reward experience per tertile of childhood adversity is presented in Table 1. 
Table 1

Means (SD) of affective symptoms by tertiles of childhood adversity and recent Stressful Life Events, and high vs. low Reward Experience.

\begin{tabular}{ccc}
\hline & \multicolumn{2}{c}{ Affective symptoms } \\
\cline { 2 - 3 } & Low Reward Experience & High Reward Experience \\
\hline Childhood adversity & & \\
Low & $1.25(.26)$ & $1.30(.30)$ \\
Medium & $1.44(.40)$ & $1.36(.33)$ \\
High & $1.62(.55)$ & $1.54(.49)$ \\
Recent Stressful life events & & $1.35(.36)$ \\
Low & $1.35(.34)$ & $1.50(.47)$ \\
Medium & $1.36(.34)$ & $1.43(.37)$ \\
High & $1.60(.57)$ &
\end{tabular}

Moderation by recent SLE

As hypothesized, the two-way interaction between recent SLE and reward experience was significant $\left(\beta=-0.08, \chi^{2}(1)=4.65, p=0.01\right)$. High reward experience was associated with less future affective symptoms after recent SLE. There was no linear dose-response relationship: reward experience was only associated with future affective symptoms in participants with a high amount of recent SLE, compared to those with a low amount $\left(\beta=-0.21, \chi^{2}(1)=7.99, p=\right.$ 0.01). A moderate amount of recent SLE was not associated with an increase in the effect of reward experience on follow-up affective symptoms $\left(\beta=0.07, \chi^{2}(1)=\right.$ $0.63, p=0.43)$. For effect sizes of baseline reward experience on follow-up affective symptoms, stratified by level of recent SLE, see Figure 2. For descriptive information on the average levels of affective symptoms for high and low levels of reward experience per tertile of recent SLE, see Table 1.

\section{Moderation by genetic risk}

The three-way interaction between affective symptoms in the co-twin, zygosity, and reward experience in the model of follow-up affective symptoms in the participant was not statistically significant $\left(\beta=0.14, \chi^{2}(1)=0.90, p=0.34\right)$. However, the two-way interaction between reward experience and co-twin affective symptoms on follow-up affective symptoms was larger in monozygotic twins $(\beta=-0.20, p=0.03)$ than in dizygotic twins (dizygotic twins: $\beta=-0.06, p=$ 0.61 ). This means that the relationship between high reward experience and less future affective symptoms appears to be more pronounced in monozygotic twins with a symptomatic co-twin than in dizygotic twins with a symptomatic co-twin, 
suggesting genetic moderation. The difference between mono- and dizygotic twins, however, is not significant.

Independence of moderation by childhood adversity and recent SLE

In order to examine to what extent the significantly associated risk factors childhood adversity and recent SLE were independent, affective symptoms were regressed on both two-way interactions (childhood adversity $\mathrm{x}$ reward experience, recent SLE $x$ reward experience) simultaneously. The effect size of childhood adversity $x$ reward experience was reduced by $24 \%$, and the effect size of recent SLE $x$ reward experience was reduced by $38 \%$. Both interaction terms were no longer significant.

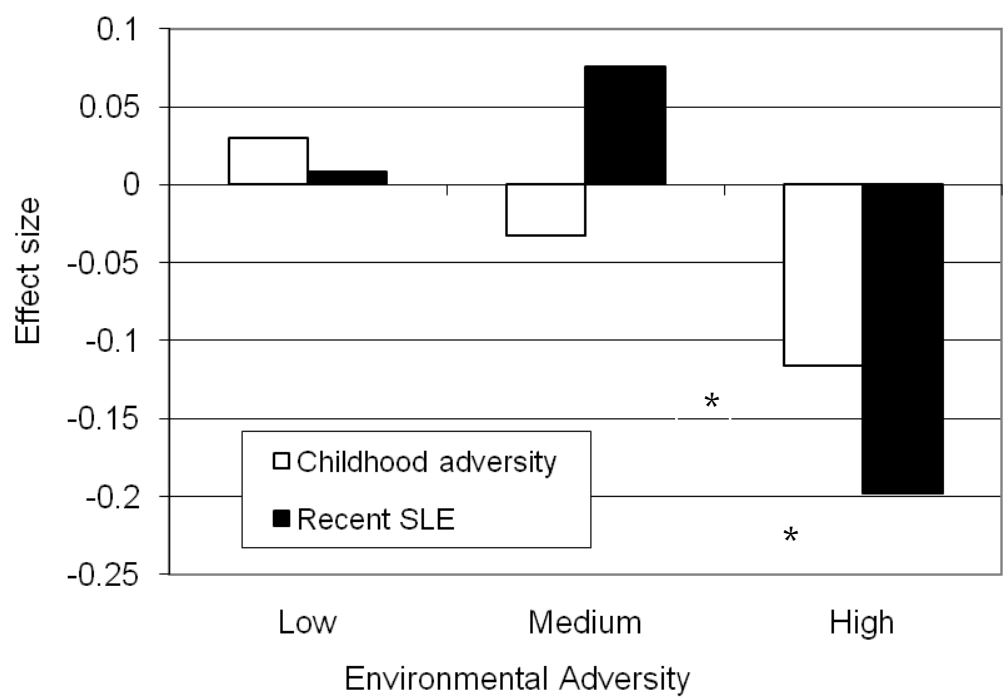

Figure 2. Effect of reward experience on follow-up affective symptoms, stratified by level of environmental adversity (childhood adversity and recent SLE, respectively). A negative effect size indicates a protective effect. Note: * represents a statistically significant difference compared to the low risk group.

Results of all analyses remained similar when additionally controlled for daily-life stress sensitivity or when participants who were depressed at baseline $(n=20)$ were excluded from the analyses. Correcting for the average number of positive events per participant and for participants' average PA did not influence our results, either. 


\section{DISCUSSION}

\section{Findings}

We hypothesized that high daily-life reward experience increases resilience against follow-up affective symptoms, and that this association is stronger in the presence of environmental adversity or genetic liability. In disagreement with the original hypothesis, there was no main protective effect of reward experience on follow-up affective symptoms. However, in line with our hypothesis, high reward experience was indeed associated with low follow-up affective symptoms in participants reporting childhood adversity or recent SLE. For childhood adversity, there was a dose-response association: with increasing levels of childhood adversity, the protective effect of high reward experience became more pronounced. For recent SLE, the protective effect of high reward experience was apparent only in the group reporting the highest number of recent SLE. Reward experience thus becomes more important in the context of increased vulnerability. The fact that people who were not or minimally exposed to environmental adversity do not need extra protection against the development of affective symptoms makes sense, since they are not likely to develop symptoms in the first place.

The experience of childhood adversity increased the likelihood of recent SLE, which is a phenomenon known from other studies (Koenen, Moffitt, Poulton, Martin, \& Caspi, 2007; Leserman, Li, Hu, \& Drossman, 1998). Although both childhood adversity and recent SLE did not contribute significantly after correcting one for the other, the effect of childhood adversity remained more stable than that of adult SLE. This suggests that early experiences may create an enduring liability that can be counteracted by resilience factors.

The three-way interaction reflecting our overall measure of genetic risk (cotwin affective symptoms $\mathrm{x}$ zygosity $\mathrm{x}$ reward experience) was not significant. This may reflect low statistical power, given the fact that three-way interactions are power-consuming. Effect sizes of reward experience, however, were associated in a dose-response fashion with level of genetic risk: high reward experience protected stronger against follow-up affective symptoms in mono- than in dizygotic twins with high-symptomatic co-twins. This is in accordance with the finding above that especially those individuals at increased risk of developing symptoms benefit from the ability to effectively generate PA in daily life. Nevertheless, new studies with more statistical power are needed to assess whether high reward experience can indeed increase resilience for people with high genetic risk for low mood. 
Additional analyses showed that the protective effects of high reward experience were independent of daily-life stress sensitivity (negative affective reactivity to unpleasant daily-life events), indicating that these represent two separate emotional pathways. Thus, reward experience, as shown previously (Wichers, et al., 2007a) as well as in the current study, appears to be involved mainly in the development of resilience whereas stress sensitivity may play a role in the development of vulnerability (Wichers, et al., 2007b; Wichers, et al., 2009c).

Furthermore, the results were unchanged by the exclusion of participants who were depressed at baseline, indicating that their depression status did not distort the results.

Results also remained similar to the original analyses when correcting for the amount of pleasant events per participant and average PA per participant, indicating that PA boosts from pleasant daily-life events contribute to resilience independently from experiencing PA in general or from encountering pleasant events. In other words, the increase in PA after pleasant events appears to be important apart from overall PA and apart from the mere frequency of pleasant events.

Taken together, our findings indicate that the ability to generate PA boosts from pleasant daily-life events increases resilience against affective symptoms in people with a history of early or late environmental adversity.

\section{Implications}

Individual variation in the ability to generate PA boosts from pleasant daily events may explain significant variation in resilience against stress-related affective disorders. Our findings are in line with a recent study of Cohn and colleagues (Cohn, et al., 2009), who found that high levels of daily PA predicted increases in trait resilience. The study of PA is therefore worthy of greater attention, complementing the main focus on negative affect. Focussing on PA should lead to novel treatment approaches and urgently needed prevention strategies against increasing numbers of stress-related disorders like depression. A recent study showed that increases in reward experience predicted recovery from depression (Wichers, et al., 2009a). This implies that the ability to generate PA boosts from pleasant daily-life events can change. With regard to the timely question of which groups should be targeted for prevention (Cuijpers \& Smit, 2008), our study suggests that efforts to prevent affective disorders should be aimed at people exposed to environmental risk factors like childhood adversity or recent stressful life events.

Regarding our concept of reward experience, a number of open questions remain: First of all, because of a delay of, on average, 90 minutes between 
consecutive beeps, there can be relatively long time delays between the recorded pleasant event and participants' report of positive affect. Therefore, high reward experience in our study may also have been a question of being more capable of maintaining PA, once activated, rather than letting it dissipate to a minimal, base level. More research is needed in order to find out exactly what constitutes the protective component in reward experience - the ability to generate PA efficiently, or the ability to maintain it for longer. Furthermore, because current PA was rated at the same time as 'pleasantness of the most important event since the last beep', beep-moment mood may have influenced ratings of event pleasantness, rather than the pleasantness of the most important event influencing mood. Future research should try to disentangle these effects, maybe by separating those questions into different beeps. A third question is whether and how - the ability to experience reward from everyday events can be enhanced. Both pharmacological as well as non-pharmacological approaches are possible.

\section{Pharmacological approaches to enhancing reward experience}

Knowledge about the biological mechanisms of reward experience is important for discovering new ways to enhance resilience. Studies suggest that dopamine projections to the prefrontal cortex are involved in the conscious experience of reward (Galvan, et al., 2005; Kringelbach, 2005; Malhi \& Berk, 2007; Schultz, 2002). The COMT enzyme, which breaks down dopamine, is closely involved in dopamine regulation in the prefrontal cortex (Bilder, Volavka, Lachman, \& Grace, 2004; Craddock, Owen, \& O'Donovan, 2006). Lower levels of COMT lead to higher prefrontal dopamine levels, and should therefore be associated with a higher ability to experience reward.

Indeed, a recent experience sampling study found that daily-life reward experience increased proportionally with the number of Met alleles on the COMT Val ${ }^{158}$ Met polymorphism (Wichers, et al., 2008a). The Met allele is associated with lower COMT activity, and, as a result, higher levels of prefrontal dopamine than the Val allele (Egan, et al., 2002). Participants with the Met/Met genotype generated comparable levels of reward experience from events rated as 'a bit pleasant' as participants with the Val/Val genotype generated from events rated as 'very pleasant' (Wichers, et al., 2008a). Similarly, participants with the Met/Met genotype had the highest prefrontal responses to anticipated and delivered rewards in a recent neuroimaging study (Dreher, Kohn, Kolachana, Weinberger, \& Berman, 2009), again indicating that individuals with Met allele experience more reward than individuals with the Val allele. 
In sum, the available evidence suggests that biological agents acting on dopamine pathways might be successful in enhancing the ability to experience reward. Although many antidepressants target the dopaminergic system (Millan, 2009), it is still unclear to what extent antidepressants improve reward experience. One recent study using imipramine (Wichers, et al., 2009a) suggests that response to antidepressants is mediated, at least in part, by changes in reward experience. However, further research is necessary to find out to what extent pharmacological interventions can improve reward experience.

\section{Non-pharmacological approaches to enhancing reward experience}

The fact that biological factors partially underlie reward experience does not exclude the possibility that non-pharmacological interventions are effective in modifying reward experience. For example, Goldapple and colleagues (2004) demonstrated that cognitive-behavioral therapy led to changes in cortical-limbic pathways in depressed patients, and Davidson and colleagues (2003) found that an 8-week mindfulness meditation program resulted in increases in activation in the left anterior hemisphere, which is associated with the experience of positive affect (Davidson, 1992; Davidson \& Fox, 1982). Furthermore, many patients prefer non-pharmacological treatment over taking medication (Van Schaik, et al., 2004). Research suggests that meditation-based techniques, such as mindfulness-based cognitive therapy (MBCT), might be an alternative way to enhance daily-life reward experience. Participation in a 7-week meditation workshop led to significant increases in PA in a non-clinical sample (Fredrickson, et al., 2008). Furthermore, more advanced meditators experienced more PA than less advanced meditators (Easterlin \& Cardena, 1998), and participants reported more PA when in a mindful compared to a non-mindful state (Brown \& Ryan, 2003).

During mindfulness training sessions, participants are trained towards increased moment-to-moment awareness of experience, resulting in increased openness or receptiveness (Baer, 2003). An enhanced receptiveness might improve the ability to make use of natural daily-life rewards. However, these suggestions remain speculative. Discovering to what extent - and how - daily-life reward experience can be enhanced therefore remains a challenging task for future research.

\section{Strengths}

Strengths of the study include the use of the experience sampling method (ESM) to establish a measure of reward experience in daily life. In contrast to conventional questionnaires or interviews, ESM measurements are prospective, repeated and cover only a short and recent time span, thereby decreasing 
memory bias (Delespaul, 1995). Furthermore, ESM provides sensitive information on the dynamic relationship between the emotional responses of an individual and his or her daily-life context.

A second strength is the study's longitudinal design, with affective symptoms being measured at baseline and at four follow-up time points. Finally, genetic risk was estimated sensitively by combining zygosity with a continuous measure of affective symptoms in the co-twin.

\section{Limitations}

First, it has been suggested that problems may arise in the ESM procedure, as it depends on participants' compliance (Kudielka, Broderick, \& Kirschbaum, 2003). In particular fixed time sampling protocols may be problematic and can bias results. However, this report did not use a fixed-time sampling frame, and our ESM procedure was validated in a previous report that used electronic monitoring devices. Results showed that compliance was high (over 90\%) and inclusion of the inaccurately timed reports did not distort the data (Jacobs, et al., 2005).

Second, reward experience was assessed prospectively but contained a cross-sectional element as the relationship between PA and pleasantness of most important event (since the last beep) was assessed at each ESM moment. Therefore, it is possible that participants matched their recall of the event to their mood at the moment of the beep, rather than pleasantness of the most important event influencing mood. However, either explanation bears clinical relevance, and the interpretation that pleasant events contribute to PA undoubtedly has face validity.

Third, childhood adversity and recent negative life events were measured retrospectively and through self-report. It is possible that those self-report measures were influenced by current mood state. However, all analyses were controlled for baseline affective symptoms, making it unlikely that results of the current study are the consequence of confounding by mood state. Moreover, the childhood trauma questionnaire has been found to have high test-retest reliability as well as good convergence with other (i.e. interview) forms of administration (Bernstein, et al., 1994), and the Interview for Recent Life Events was designed specifically to collect datable occurrences involving changes in the external social environment rather than internal occurrences (Paykel, 1997).

A fourth limitation is that the sample was female only. Consequently, our findings cannot be generalized to the male population. Furthermore, participants were mainly of middle age and, on average, higher educated than the general Belgium population. 


\section{Acknowledgements}

Funding/Support: This research was supported by the Dutch Organisation for Scientific Research (VENI grant nr 916.76.147 to Dr Wichers) and by the Fund for Scientific Research, Flanders and Twins, a Belgian non-profit association for scientific research in multiple births (grant to the East Flanders Prospective Survey). 



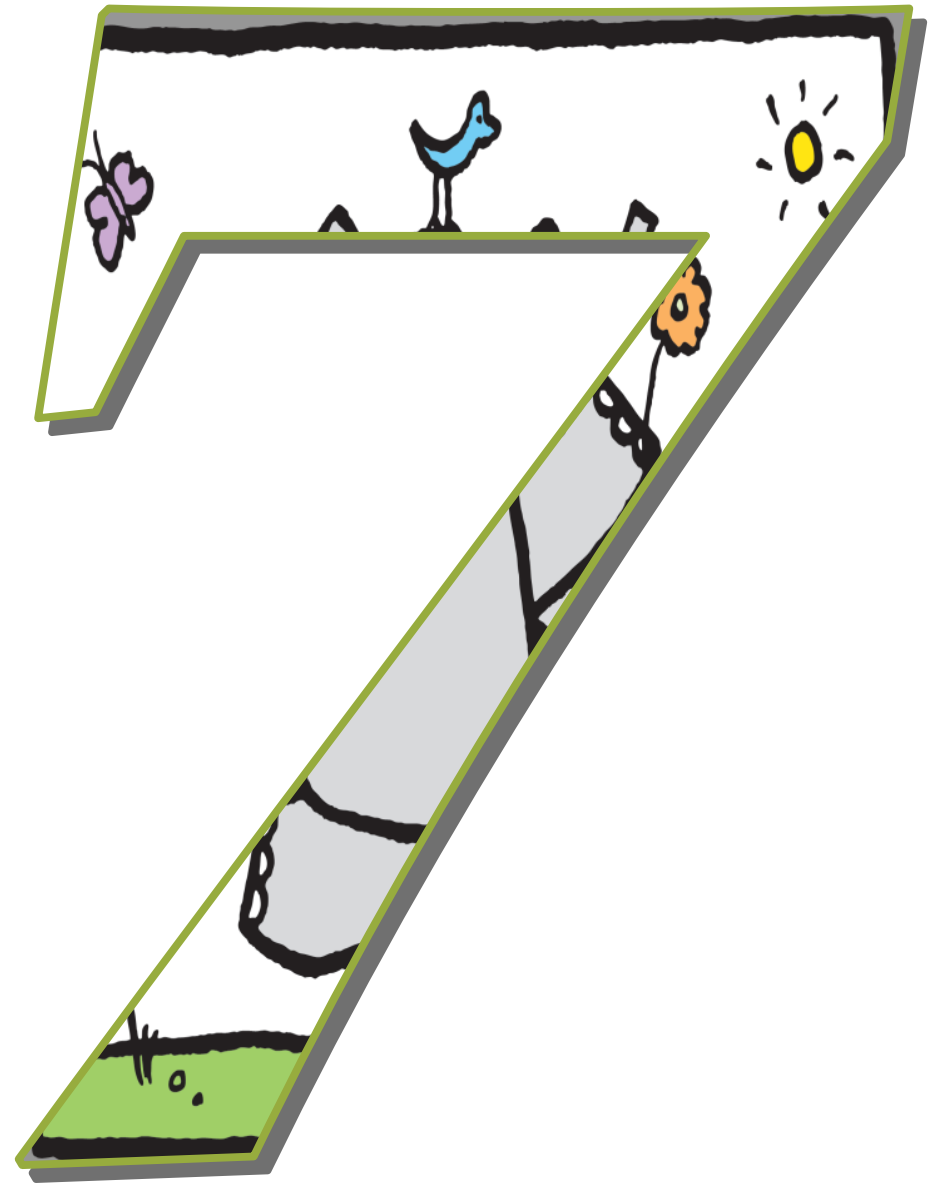

Mindfulness training increases momentary positive emotions and reward experience in adults vulnerable to depression. A randomized controlled trial 
Objective: To examine whether Mindfulness-Based Cognitive Therapy (MBCT) increases momentary positive emotions and the ability to make use of natural rewards in daily life.

Method: Adults with a life-time history of depression and current residual depressive symptoms (mean age 43.9 years, SD 9.6; $75 \%$ female; all Caucasian) were randomized to MBCT $(n=64)$ or waitlist control (CONTROL; $n=66$ ) in a parallel, open-label, randomized controlled trial. The Experience Sampling Method was used to measure momentary positive emotions as well as appraisal of pleasant activities in daily life during six days before and after the intervention. Residual depressive symptoms were measured using the 17-item Hamilton Depression Rating Scale.

Results: MBCT compared to CONTROL was associated with significant increases in appraisals of positive emotion $\left(b^{*}=.39\right)$ and activity pleasantness $\left(b^{*}=.22\right)$, as well as enhanced ability to boost momentary positive emotions by engaging in pleasant activities $\left(b^{*}=.08\right.$; all $\left.p<.005\right)$. Associations remained significant when corrected for reductions in depressive symptoms, or for reductions in negative emotion, rumination and worry. In the MBCT condition, increases in positive emotion variables were associated with reduction of residual depressive symptoms (all $\mathrm{p}<.05$ ).

Conclusions: MBCT is associated with increased experience of momentary positive emotions, greater appreciation of, and enhanced responsiveness to pleasant daily-life activities. These changes were unlikely to be pure epiphenomena of decreased depression and, given the role of positive emotions in resilience against depression, may contribute to the protective effects of MBCT against depressive relapse.

Keywords: MBCT; mindfulness-based cognitive therapy; residual symptoms; positive affect; ecological momentary assessment

Published as:

Geschwind, N., Peeters, F., Drukker, M., van Os, J., \& Wichers, M. (2011). Mindfulness training increases momentary positive emotions and reward experience in adults vulnerable to depression: A randomized controlled trial. Journal of Consulting and Clinical Psychology, 79(5), 618-628. doi: 10.1037/a0024595 
Major depression is a common and highly recurrent clinical condition - an estimated 75 to 90 percent of patients will have multiple episodes (e.g., Mueller, et al., 1999). Even after different treatment steps a considerable number of depressed participants continue to experience residual symptoms of depression (Rush, et al., 2006). Residual symptoms not only impact negatively on quality of life, they are also associated with three times faster relapse, compared to full recovery (Judd, et al., 1998; Rush, et al., 2006). Residual symptoms commonly include sleep disturbances, loss of energy, and decreased pleasure, motivation and interest. Overall, this pattern of symptoms is consistent with lower levels of positive affect (Nutt, et al., 2007; Watson, Clark, \& Carey, 1988) - a core symptom of Major Depressive Disorder. Previous studies suggest that positive affect promotes prevention of and recovery from depression (Geschwind, et al., 2011a; Morris, Bylsma, \& Rottenberg, 2009; Wichers, et al., 2010). Prevention efforts focused on increases in positive affect are therefore likely to (a) improve residual symptoms and well-being, and (b) prevent relapse.

\section{POSITIVE AFFECT, WELL-BEING, AND DEPRESSION}

Experiencing positive emotions and having a positive attitude have beneficial effects on mental and physical health in general (Seligman, et al., 2005). A metaanalysis of cross-sectional, longitudinal and experimental studies demonstrated that positive affect was associated with and preceded success, indicating that positive affect facilitates accomplishment (Lyubomirsky, et al., 2005). Evidence also suggests that positive affect is a source of resilience against pain and negative affectivity. For example, in a study in which women with chronic pain were interviewed weekly, higher levels of positive affect predicted lower levels of pain in subsequent weeks. Higher weekly levels of positive affect also resulted in lower levels of negative affect both directly and in interaction with stress and pain (Zautra, et al., 2005). A recent study suggests that especially in-the-moment positive emotions, rather than more general satisfaction with life, are associated with increased resilience (Cohn, et al., 2009). Momentary assessment studies, in which experiences are sampled repeatedly at random moments in daily life, support this view. For example, high daily-life reward experience (i.e., the ability to generate positive affect from pleasant daily events) predicted increased resilience against later affective symptoms in participants vulnerable to depression (Geschwind, et al., 2010; Wichers, et al., 2010). Furthermore, in participants with a lifetime history of depression, scoring one standard deviation higher on the ability to generate positive affect from pleasant daily-life events was 
associated with a three-fold reduction in risk to experience a future episode (Wichers, et al., 2010).

Studies show that depressed participants, compared to never-depressed controls, experience less positive affect in the course of daily life (BargeSchaapveld, Nicolson, Berkhof, \& deVries, 1999; Bylsma, Taylor-Clift, \& Rottenberg, 2011; Peeters, Berkhof, Delespaul, Rottenberg, \& Nicolson, 2006). Also, they generate less positive affect from pleasant stimuli during experimental tasks (Bylsma, Morris, \& Rottenberg, 2008), though in daily life this phenomenon is debatable (Bylsma, et al., 2011; Peeters, et al., 2003).

Taken together, the evidence suggests that (a) high levels of positive affect have beneficial effects on vulnerability to, prevention of, and recovery from depression, and that (b) depressed individuals generate less positive affect, compared to non-depressed individuals. In other words, positive affect may represent a resilience phenotype against depression. Individuals vulnerable to depression may therefore benefit from learning to experience more positive affect. The question arises how people can reshape emotional processes in a way that heightens their ability to experience positive affect in their daily lives. One momentary assessment study showed that response to a six-week treatment with antidepressant medication was associated with a heightened ability to boost positive affect through pleasant activities, relative to baseline (Wichers, et al., 2009). However, advocating use of antidepressant medication for prevention purposes only is problematic - and whether a behavioral, non-pharmacological, intervention can be used to heighten participants' ability to generate positive emotions in daily life has never been tested.

An intervention currently receiving empirical support for the prevention of depressive relapse and recurrence, and for the treatment of residual depressive symptoms, is Mindfulness-Based Cognitive Therapy (MBCT; Segal, Williams, \& Teasdale, 2002b). Although many authors assume changes in cognitive processes to be a core element of the beneficial effects of MBCT (Segal, Teasdale, Williams, \& Gemar, 2002a), it is unclear if and how MBCT affects alterations in emotional experiences. As we argue below, there is reason to believe that MBCT may have the potential to induce changes in resilience phenotypes associated with positive affect.

\section{MINDFULNESS-BASED COGNITIVE THERAPY}

During mindfulness exercises, participants attempt to maintain attention on a particular focus, for example their own breathing. Whenever the attention 
wanders away from breathing to thoughts or feelings, participants are encouraged to acknowledge and accept these thoughts and feelings but then let go of them and direct their attention back to breathing. Participants then use the same approach in the course of daily life: they aim to focus on the 'here and now' (to engage with the present experience) and to redirect their attention whenever they notice that it has switched to distracting thoughts and worries. Next to this attentional aspect, mindfulness also works on an attitudinal dimension by promoting the cultivation of an open, curious and mild orientation of mind. The essence of a mindful state is to be fully in the present moment, to experience the present moment without judgment or evaluation and without worrying about the future or ruminating about past experiences (Kabat-Zinn, 1990).

Mindfulness-Based Cognitive Therapy (MBCT) is specifically designed to prevent depressive relapse and recurrence. MBCT combines methods of meditation and mindfulness training (Kabat-Zinn, 1990) with features of cognitive therapy for depression (Beck, et al., 1979; Teasdale, et al., 1995). The original idea behind $\mathrm{MBCT}$ was to train participants to disengage from automatic negative thinking patterns that arise during dysphoric mood and facilitate relapse (Teasdale, et al., 2000). Several studies have shown that MBCT is associated with reduced depressive symptoms and lower risk of relapse (Bondolfi, et al., 2010; Kuyken, et al., 2008; Ma \& Teasdale, 2004; Teasdale, et al., 2000). Furthermore, recent studies found that $\mathrm{MBCT}$ is associated with reduced cognitive reactivity (Kuyken, et al., 2010; Raes, Dewulf, Van Heeringen, \& Williams, 2009), rumination (Williams, 2008), and worry (Evans, et al., 2008).

However, there is reason to believe that MBCT might also increase participants' capacity for the experience of positive affect (Garland, et al., 2010; Geschwind, et al., 2010). Studies show that more advanced meditators experience more positive emotions (Easterlin \& Cardena, 1998), and that people report more positive emotions when in a mindful compared to a non-mindful state (Brown \& Ryan, 2003; Killingsworth \& Gilbert, 2010). Furthermore, in a randomized controlled trial, participants of loving-kindness meditation (LKM) reported stronger increases in positive affect over time, compared to control participants (Fredrickson, et al., 2008). During LKM participants practice to experience love and compassion first towards themselves and then towards loved ones, acquaintances, and strangers (Fredrickson, et al., 2008). This very explicit focus on positive emotions may, however, create a demand bias, which is much less pronounced in MBCT. Although awareness of pleasant events and nourishing activities is addressed during two to three of the eight training sessions (just as reactions to stressful situations are dealt with), the main focus during MBCT 
sessions is to develop an increased moment-to-moment awareness of experience (Baer, 2003). As pleasant events and emotions are usually less enduring, intense and attention-grabbing than unpleasant events and emotions (Baumeister, Bratslavsky, Finkenauer, \& Vohs, 2001), they may be more easily overlooked than their unpleasant counterparts. Increased moment-to-moment awareness, achieved in a mindful state, may help people to perceive fleeting pleasant events or emotions and to generate more enjoyment from pleasant activities (Schroevers \& Brandsma, 2010).

\section{HYPOTHESES}

Positive affect is associated with increased resilience against depression, but it remains unclear to which extent phenotypes related to positive affect can be modified trough behavioral interventions. We propose that MBCT is an intervention that increases the ability to make use of natural, moment-tomoment rewards in the environment, thereby increasing positive affect. Our primary hypothesis is that, after $\mathrm{MBCT}$, participants (a) experience more positive affect, (b) rate pleasant daily-life activities as more pleasant, and (c) experience more reward (i.e., generate more positive affect from engaging in pleasant activities). Because previous literature has shown that MBCT is associated with decreased worry, rumination (see above) and negative affect (Schroevers \& Brandsma, 2010), we will additionally examine to which extent potential changes in positive affect, pleasant activities, and reward experience are independent of decreases in worry, rumination and negative affect. Because of the protective links between positive affect and depression, a secondary hypothesis is that increases in positive affect, pleasant activities, and reward experience will be associated with decreases in depressive symptoms within the MBCT condition.

Hypotheses were tested in an open-label, parallel, randomized controlled trial comparing participants who continued with treatment as usual to participants who, additionally, received $\mathrm{MBCT}$. To measure the fleeting momentary experience of positive emotions (Garland, et al., 2010) in an ecologically valid and reliable manner, the Experience Sampling Method (ESM) was used. ESM is a momentary assessment technique in which participants are prompted to report on their current experiences at random moments during the day. ESM is therefore ideally suited to investigate changes in people's emotional reactions to their daily environment (Csikszentmihalyi \& Larson, 1987). To our knowledge, this is the first study to examine how MBCT is associated with changes in people's momentary affective responses in their daily-life context. 
Furthermore, this trial is the first to examine whether a non-pharmacological intervention can be used to modify a resilience phenotype (the ability to experience and generate positive emotions in daily life) in a sample vulnerable to depression.

\section{METHOD}

\section{Participant Characteristics}

For the current study (acronym MindMaastricht; trial number NTR1084, Netherlands Trial Register), adults with residual symptomatology after at least one episode of Major Depressive Disorder were recruited from outpatient mental health care facilities in Maastricht and through posters in public spaces. Residual symptoms are associated with higher risk of relapse (Judd et al., 1998) and were therefore required as an indicator of vulnerability to depression. Residual symptoms were defined as a score of seven or higher on the 17-item Hamilton Depression Rating Scale (HDRS; Hamilton, 1960) at the time of screening. Exclusion criteria were: fulfilling criteria for a current depressive episode, schizophrenia, psychotic episodes in the past year, and recent (past four weeks) or upcoming changes in ongoing psychological or pharmacological treatment. Currently depressed individuals were excluded because, at trial preparation, there was no evidence that currently depressed individuals were able to participate in or benefit from MBCT. Relevant sociodemographic and clinical characteristics are displayed in Table 1.

\section{Sampling procedures}

All study procedures were approved by the Medical Ethics Committee of Maastricht University Medical Centre, and all participants signed an informed consent form. An initial screening of potential participants for this randomized controlled trial was performed by phone to check for availability during the study period and likelihood of meeting in- and exclusion criteria. A second screening included administration of the Structured Clinical Interview for DSM IV axis I (SCID-I; First, Spitzer, Gibbon, \& Williams, 2002) and the 17-item HDRS by trained psychologists. Eligible participants were invited for a detailed one-on-one explanation of the experience sampling procedure, and then took part in the baseline assessment. The baseline assessment consisted of six days of experience sampling in their own environment (see section Experience Sampling Method; ESM), and subsequent administration of a battery of questionnaires (see section Measures) as well as the HDRS interview (in the laboratory). After the baseline assessment, participants were randomized to either MBCT or waiting list 
(CONTROL; allocation ratio 1:1) if they were likely to have at least 20 valid ESM assessments (Delespaul, 1995; see Experience Sampling Method). After either eight weeks of MBCT (see section "Intervention") or equivalent waiting time (in the CONTROL condition), participants again took part in six days of experience sampling, followed by the administration of the HDRS and the questionnaires. All participants were compensated with gift vouchers worth 50 Euros. Participants in the CONTROL condition had the opportunity to take part in MBCT after the postintervention assessment.

Randomization to treatment condition was stratified according to number of depressive episodes (two or less versus three or more), as previous studies suggest a greater benefit for those with three or more previous episodes (Ma \& Teasdale, 2004; Teasdale, et al., 2000). An independent researcher not involved in the project generated the randomization sequence in blocks of five (using the sequence generator on www.random.org), and wrote the randomization code into sealed numbered envelopes. After completion of all baseline assessments, the researcher allocated participants to their treatment condition based on the randomization code in the sealed envelope (opened in order of sequence). No masking of treatment condition took place.

\section{Sample Size and Power}

Sample size $(n \geq 120)$ was determined on the basis of sufficient power for geneenvironment interactions (not analyzed here). Post-hoc power calculations for the current analyses (using Stata 11's SAMPSI command; StataCorp, 2009) indicated a power of $>.90$ to detect small effects $(d=0.2)$ in the parameter of interest, the group*time interaction.

\section{Intervention (MBCT)}

Content of MBCT training sessions followed the protocol of Segal, Williams, and Teasdale (2002). Trainings consisted of eight weekly meetings lasting 2.5 hours and were run in groups of 10-15 participants (thus occasionally larger than the usual 10-12 participants per group). Assessment periods for control participants were matched to those of MBCT participants. Sessions included guided meditation, experiential exercises, and discussions. In addition to the weekly group sessions, participants received CDs with guided exercises and were assigned daily homework exercises (30 to 60 minutes daily). Trainings were given by experienced trainers in a centre specialized in mindfulness trainings. All trainers were supervised by an experienced health care professional who had trained with Teasdale and Williams, the co-developers of MBCT (Teasdale, et al., 1995). 


\section{Experience Sampling Method}

ESM is a momentary assessment method to assess participants in their daily living environment, thus providing repeated in-the-moment assessments of affect in a prospective and ecologically valid manner (Csikszentmihalyi \& Larson, 1987; Peeters, et al., 2003). Compared to retrospective questionnaires and interviews, ESM offers several advantages: (a) enhanced ecological validity, because participants are assessed in their normal daily environments, (b) minimized retrospective bias, because participants' experiences are assessed in the moment, and (c) enhanced reliability, because participants' experiences are assessed repeatedly (Csikszentmihalyi \& Larson, 1987).

In the current study, participants received a digital wristwatch and a set of ESM self-assessment forms collated in a booklet for each day. The wristwatch was programmed to emit a signal ("beep") at an unpredictable moment in each of ten 90-minute time blocks between 7:30 am and 10:30 pm, on six consecutive days, resulting in a maximum of 60 beeps per study period. After each beep, participants were asked to fill out the ESM self-assessment forms previously handed to them, collecting reports of current mood and context. All selfassessments were rated on 7-point Likert scales. Trained research assistants explained the ESM procedure to the participants during an initial briefing session, and a practice form was completed to confirm that participants understood the 7point Likert scale. Participants were instructed to complete their reports immediately after the beep, thus minimizing memory distortion, and to record the time at which they completed the form. All reports not filled in within $15 \mathrm{~min}$ after the actual beep were excluded from the analysis, since previous work (Delespaul, 1995) has shown that reports completed after this interval are less reliable and consequently less valid. For the same reason, participants with less than 20 valid reports at baseline were excluded from the analysis (Delespaul, 1995).

\section{Measures}

Pleasantness of daily-life activities.

To define pleasantness of daily-life activities in an ecologically valid manner, ESM self-rated appraisals of ongoing activities were used, consistent with several previous studies of emotional reactivity to appraised daily activities and contexts (Myin-Germeys \& van Os, 2007; Myin-Germeys, et al., 2001; Wichers, et al., 2009a). Participants rated their current activity on a 7-point Likert scale (with $1=$ not at all and $7=$ very). Factor analysis supported inclusion of four items for activity appraisal (with factor loadings $>.6$ ), namely: I enjoy this activity, This 
activity requires effort, I would prefer to do something else and I am skilled at doing this activity. Two items (I feel I'm being active and This is a challenge) had low factor loadings (.05 and .12, respectively), and were consequently not included in the activity pleasantness score. On the basis of the included ratings, a variable reflecting 'pleasantness of current activity' was generated. Before creating the activity pleasantness variable, the items 'This activity requires effort' and ' $I$ would prefer to do something else' were first recoded so that high scores reflected lower appraised effort and higher preference for the current activity. Consistent with Wichers and colleagues (2009), low scores ( $\leq 4)$ on all four items were set to zero (so that negative activity appraisals did not contribute to the overall score), and higher scores were recoded $(5=1,6=2,7=3)$ before calculating a sum score for activity pleasantness. High pleasantness thus reflected high skill, low effort, low preference for doing something else, and high enjoyment of the activity.

Positive affect (PA) and negative affect (NA).

At each beep, several ESM mood adjectives were assessed on 7-point Likert scales ranging from 1 (not at all) to 7 (very). Consistent with previous work (MyinGermeys, et al., 2001; Wichers, et al., 2010), principal component factor analysis with oblique rotation was used to generate a factor representing positive affect (PA) and a factor representing negative affect (NA). The mood adjectives happy, satisfied, strong, enthusiastic, curious, animated and inspired loaded on the PA factor (alpha = .89), while down, anxious, lonely, suspicious, disappointed, insecure and guilty loaded on the NA factor (alpha $=0.86$ ). One mood item (I feel relaxed) was not included in the PA factor due to low factor loadings (<.6). Mean levels of PA and NA were then computed per participant and beep moment.

Reward experience.

Reward experience was conceptualized as the effect (the standardized coefficient $b^{*}$ ) of pleasant activities on momentary PA. This coefficient captures the increase in PA when engaging in pleasant activities, relative to baseline. Reward experience thus was not a precalculated variable but hidden in the outcome of the analyses (consistent with Wichers, et al., 2009), so there was no way in which participants could consciously fake the outcome on this variable.

Hamilton Depression Rating Scale (HDRS).

The 17-item HDRS was administered by two trained research assistants with Master degrees in Psychology. The HDRS (Hamilton, 1960) is a semi-structured interview designed to assess depressive symptoms over the past week. It is one of the most often used rating scales in depression research, and internal, interrater, 
and retest reliability estimates for the overall HDRS are good (Bagby, et al., 2004). Only the overall score was used for the analyses, and interrater reliability for the total score was high (IntraClass Correlation coefficient $=.97$ ). To provide information on interrater reliability, both interviewers had independently rated eight video-taped HDRS interviews with patients varying in strength of residual depressive symptoms.

Inventory of Depressive Symptoms (Self-Rating; IDS-SR).

The IDS-SR (Rush, Gullion, Basco, Jarrett, \& Trivedi, 1996) is a self-rated scale, which includes 30 items rated on a scale from 0 to 3 . Since rating of 'appetite' and 'weight' is duplicated (separate items for increases and decreases), only 28 items are taken into account for the final score. The scale is sensitive to change and has good psychometric properties (Rush, et al., 1996). Internal consistency in the current sample was .85 .

Penn State Worry Questionnaire (PSWQ).

A Dutch version of the 16-item PSWQ was used to assess worrying. The PSWQ emerged from factor analysis of a large number of items and was found to possess high internal consistency and good test-retest reliability (Meyer, Miller, Metzger, \& Borkovec, 1990). Internal consistency in our sample was .90.

Rumination on Sadness Scale (RSS).

The approved Dutch back-translation (Raes, Hermans, \& Eelen, 2003) of the RSS (Conway, Csank, Holm, \& Blake, 2000) was used to measure rumination. The RSS contains 13 items comprising one factor, has an adequate test-retest stability over a 2- to 3-week period, and has good convergent and discriminant validity (Conway, et al., 2000). Internal consistency in the current sample was .90, similar to earlier studies (e.g., Conway, et al., 2000).

\section{Statistical Methods}

Experience Sampling Method data have a hierarchical structure. Thus, multiple observations (level 1) are clustered within participants (level 2). Multilevel analyses take the variability associated with each level of nesting into account (Snijders \& Bosker, 1999). Multilevel linear regression analyses, using the XTREG command in STATA 11.1 (StataCorp, 2009) were applied to the ESM data. 'Reward Experience' was conceptualized as the effect of positively appraised daily-life activities on PA (i.e., the boost in PA when participants engage in more pleasant compared to neutral activities). 
First, we examined the effects of MBCT on change in positive affect, pleasantness of activities, and reward experience, relative to CONTROL. Thus, in the models of PA and activity pleasantness, the two-way interaction between time (baseline vs. post assessment) and treatment group (CONTROL vs. MBCT) was the parameter of interest. For reward experience, the three-way interaction between time (baseline vs. post assessment), treatment group (CONTROL vs. $\mathrm{MBCT}$ ), and activity pleasantness in the model of PA was the parameter of interest. The MARGINS, DYDX command (StataCorp, 2009) was used to calculate the effect of activity pleasantness on PA in the four different conditions (MBCT and CONTROL, at baseline and post-intervention). Per treatment group, significance of differences between baseline and post-assessment were then assessed with Stata's TEST command, which uses the Wald test (Clayton \& Hill, 1993). To refute concerns that improvements in PA-related variables may simply reflect epiphenomena of decreased depression scores, analyses were reran posthoc whilst correcting for reduction on the HDRS.

Second, to examine to what extent the effect of treatment on PA, pleasant activities, and reward experience was independent of changes in third variables known from MBCT research, the above-mentioned analyses were repeated whilst including the interaction terms containing changes in worry, rumination, and NA (i.e., time*worry + time*rumination + time*NA).

Third, we examined to what extent MBCT-related improvements in the variables PA, activity pleasantness, and reward experience were associated with decreases in depressive symptoms. For this reason, analyses for all three variables were repeated in the MBCT group only, using "Reduction on the HDRS" (baseline minus post assessment) in the interaction terms instead of treatment group. To visualize these associations more clearly, and to examine dose-response associations between improvements in PA-related variables and depressive symptoms, we then divided improvement on the HDRS into tertiles. The strength of associations was tested using the margins command, and significance was assessed using the Wald test (Clayton \& Hill, 1993). To ensure that these associations were independent of the format of measurement, the analyses were repeated using the self-report IDS-SR to classify change in depressive symptoms (instead of the clinician-rated HDRS).

Because average levels at baseline naturally influence the extent to which a variable can increase (e.g., ceiling effects), the analyses on PA and reward experience were corrected for individually averaged baseline levels of PA. Similarly, the analyses on activity pleasantness were corrected for averaged baseline ratings of activity pleasantness. 
The reported analyses are based on the whole sample (intention to treat). Participants who attended less than four MBCT sessions $(n=3)$ were excluded for the per-protocol analysis (outcomes were similar and are not reported in detail).

\section{RESULTS}

\section{Participants}

Recruitment of participants started in January 2008 and ended in February 2009, and all post-intervention assessments were completed by August 2009, when the pre-determined number of participants was reached. Sociodemographic and clinical characteristics of MBCT and CONTROL participants are displayed in Table 1. At baseline, there were no large or significant differences between treatment groups with respect to sociodemographic and clinical characteristics. Table 2 shows baseline and post assessment scores of variables used in the analyses, stratified by treatment group. Again, there were no large or significant differences between groups at baseline. Participant flow through the study is displayed in Figure 1. No known harms or unintended treatment effects were reported in either group.

Participants completed 12,453 entries in total. Of these, 559 (4 \%) were excluded as invalid entries, because completion times fell outside the predetermined window of 15 minutes after the beep. On average, participants completed 49 (of 60; SD = 7.6) valid entries per assessment period. One participant had completed fewer than 20 valid entries at baseline and was therefore excluded from the analyses.

\section{Effects of MBCT on PA-related variables}

MBCT compared to CONTROL was associated with significant increases in PA, $\mathrm{b}^{*}=$ $.40,95 \% \mathrm{Cl}[.33, .46], \chi^{2}(1)=163.02, p<.001$. Similarly, MBCT was associated with increases in activity pleasantness, compared to CONTROL, $b^{*}=.22,95 \% \mathrm{Cl}$ $[.15, .29], \chi^{2}(1)=39.09, p<.001$. Also reward experience increased significantly in the MBCT group, compared to CONTROL, $b^{*}=.08,95 \% \mathrm{Cl}[.03, .14], \chi^{2}(1)=8.23$, $p=.004$. Standardized predicted values of PA-related variables, by group and assessment period, are displayed in Figure 2. Effects of treatment condition on PA-related variables were similar when the analyses were repeated whilst correcting for change in residual depressive symptoms $\left(b^{*}=.40, .22\right.$, and .08 for $\mathrm{PA}$, activity pleasantness, and reward experience, respectively), indicating that the effects of MBCT on PA-related variables were not just epiphenomena of reduction in depressive symptoms. 


\section{Independence of effects of MBCT on PA-related variables}

As can be seen in Table 2, MBCT was also associated with significant reductions in worry, rumination, and NA, compared to CONTROL. However, effects of MBCT on PA-related variables were reduced but remained evident and statistically significant when changes in worry, rumination, and NA were included in the analyses. The effect size of the time (baseline vs. post-assessment) by group (MBCT vs. CONTROL) interaction term then became $b^{*}=.19, p<.001$ (unadjusted $b^{*}=.40$ ) in the model of PA, and $b^{*}=.16, p<.001$ (unadjusted $b^{*}=.22$ ) in the model of activity pleasantness. In the model of reward experience the effect size of the three-way interaction term time*group*activity pleasantness became $b^{*}=$ $.07, p=.007$ (unadjusted $\mathrm{b}^{*}=.08$ ).

\section{Clinical Significance: Association between symptom reduction and PA-related increases}

With regard to clinical significance, analyses restricted to the MBCT group showed that reduction on the HDRS was associated with significant increases in PA, b* = $.30,95 \% \mathrm{Cl}[.26, .34], p=.000$, activity pleasantness, $\mathrm{b}^{*}=.05,95 \% \mathrm{Cl}[.002, .10], p$ $=.000$, and reward experience, $\mathrm{b}^{*}=.06,95 \% \mathrm{Cl}[.02, .09], p=.003$. In order to examine dose-response relationships, participants were classified as low ( $n=21$, mean reduction in HDRS scores $\triangle M=-1.94, S D=2.83$, range -9 to 1$)$, medium $(n=$ 22 , HDRS $\Delta M=3.89, S D=1.02$, range $2-5)$, and high responders $(n=20$, HDRS $\Delta M=8.31, S D=1.53$, range $6-12$ ) to $M B C T$, using a tertile split. Figure 3 displays standardized predicted values of PA-related variables by tertiles of response and assessment period (pre vs. post MBCT), confirming the predicted pattern of results. Results were similar when the IDS-SR was used to assess symptom reduction instead of the HDRS. 
Table 1

Baseline demographic and clinical characteristics per group.

\begin{tabular}{lcc}
\hline & $\begin{array}{c}\text { MBCT } \\
(n=63)\end{array}$ & $\begin{array}{c}\text { CONTROL } \\
(n=66)\end{array}$ \\
\hline Age (M, SD) & $44.6(9.7)$ & $43.2(9.5)$ \\
Female gender & $79 \%$ & $73 \%$ \\
Full-/part-time work & $62 \%$ & $68 \%$ \\
Illness/unemployment benefits & $19 \%$ & $23 \%$ \\
Living with partner/own family & $64 \%$ & $64 \%$ \\
$\leq 2$ previous episodes of MDD & $56 \%$ & $55 \%$ \\
$\geq 3$ previous episodes of MDD & $44 \%$ & $45 \%$ \\
Comorbid anxiety disorder (present) & $35 \%$ & $49 \%$ \\
Comorbid anxiety disorder (past) & $51 \%$ & $64 \%$ \\
Current psycho-counseling/-therapy & $13 \%$ & $12 \%$ \\
Current use of antidepressants & $32 \%$ & $38 \%$ \\
(Occasional) use of benzodiazepines & $8 \%$ & $8 \%$ \\
\hline
\end{tabular}

Note. There were no significant differences between groups (at $\mathrm{p}<.05) . \mathrm{MBCT}=$ Mindfulness-Based Cognitive Therapy; $\mathrm{CONTROL}=$ waitlist control condition; $\mathrm{MDD}=$ Major Depressive Disorder. 
Table 2

Means and standard deviations of variables used in the analyses, stratified by group and measurement occasion.

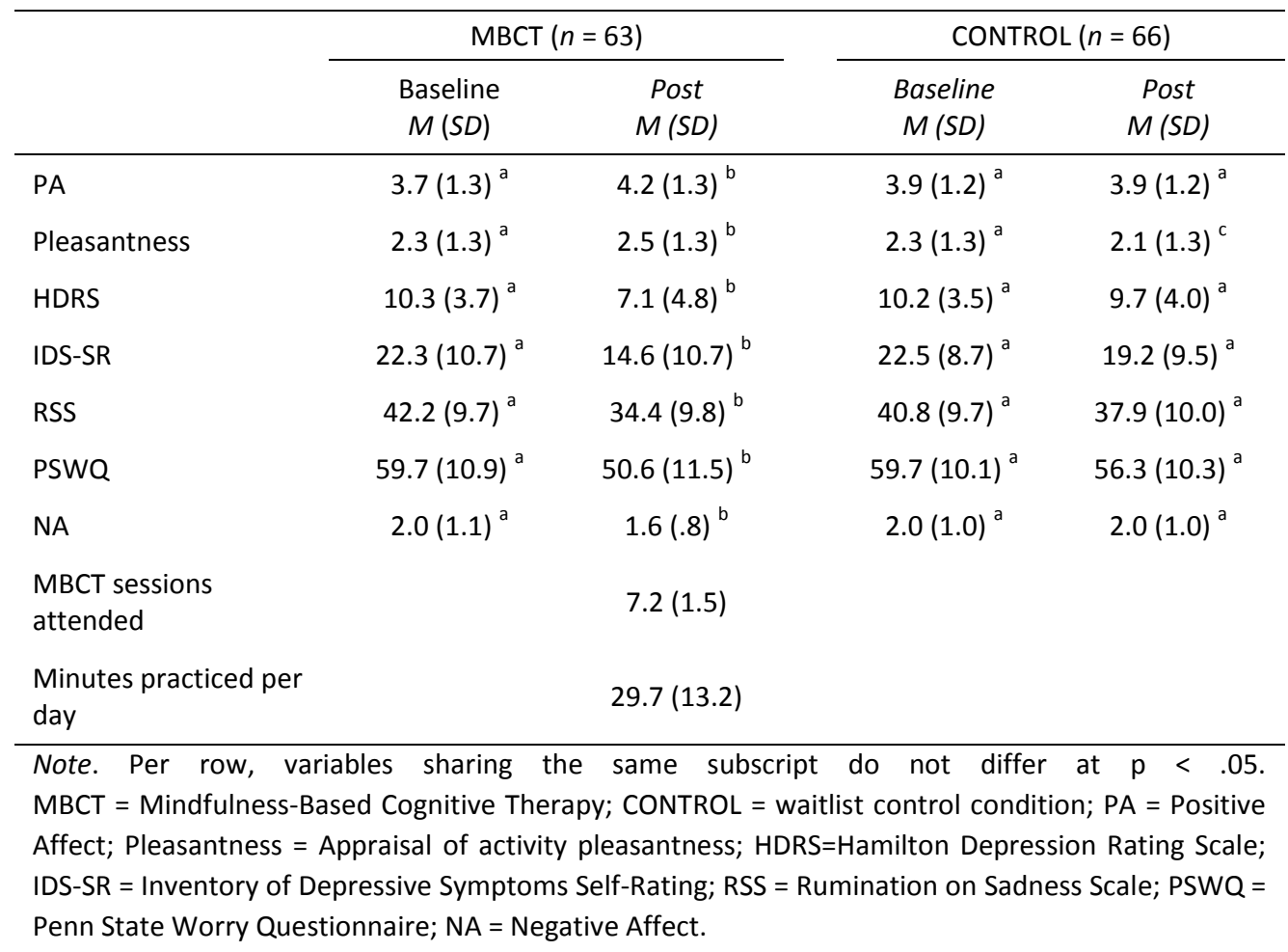




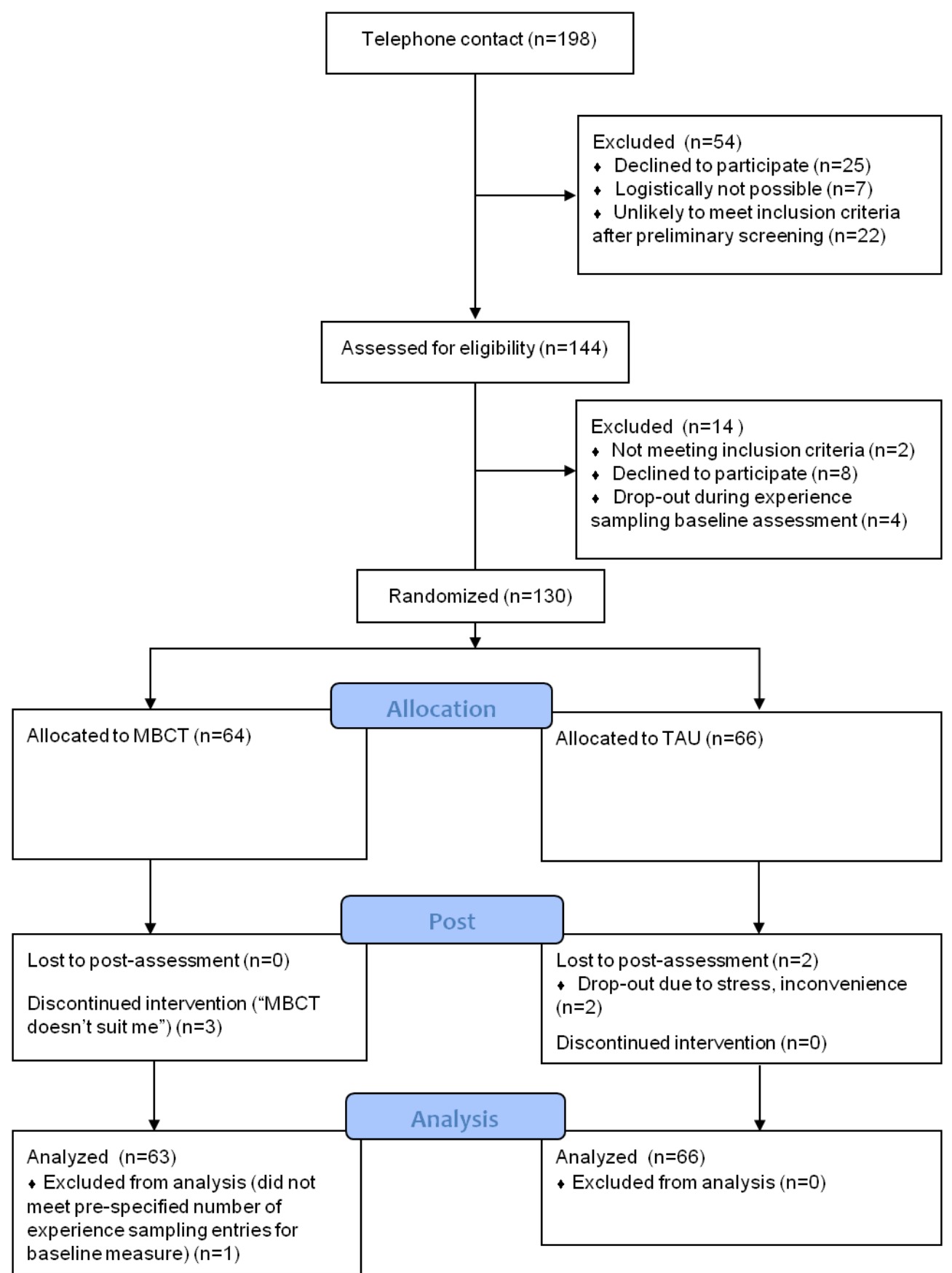

Figure 1. Participant flow diagram. MBCT = Mindfulness-Based Cognitive Therapy; CONTROL = waitlist control condition. 


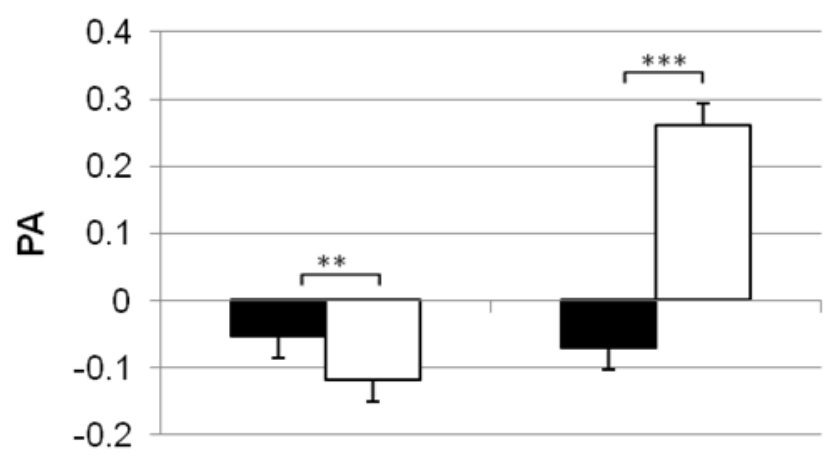

baseline $\square$ post

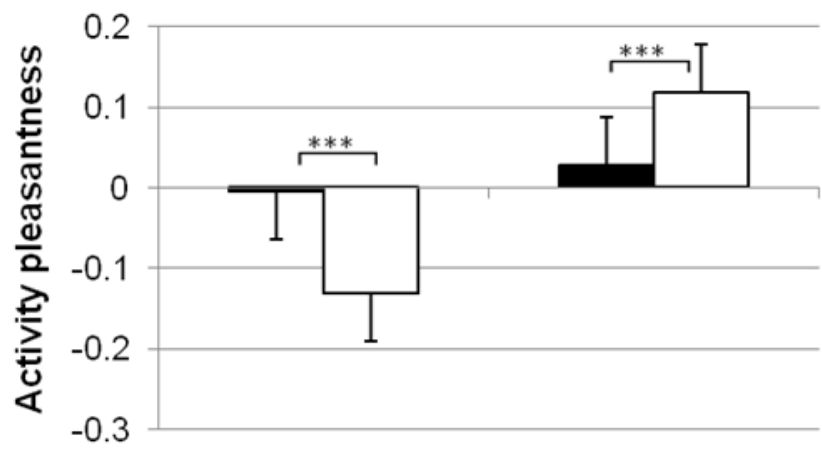

baseline $\square$ post

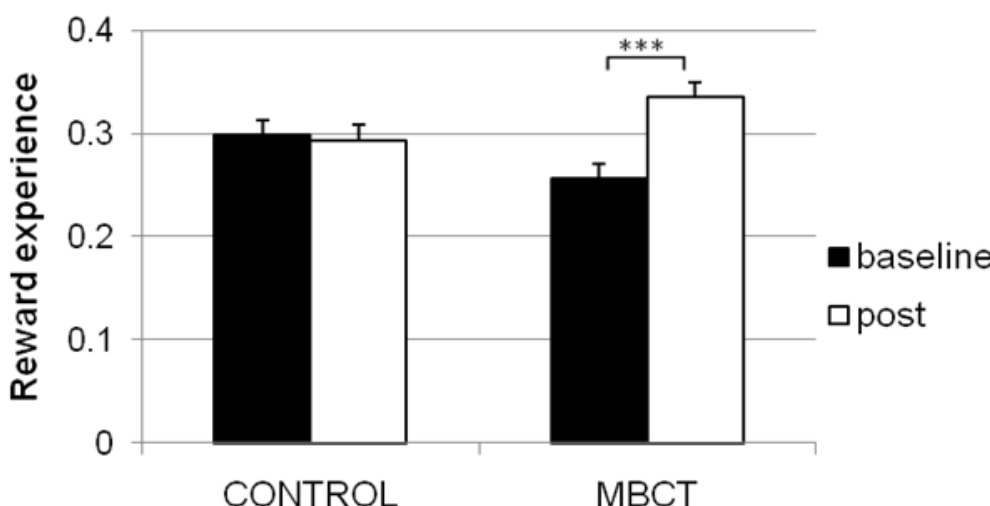

Figure 2. Standardized predicted values of positive affect, activity pleasantness, and reward experience $( \pm \mathrm{SE})$ at baseline and post-assessment in CONTROL and MBCT groups. Analyses were controlled for a person's mean baseline level on the dependent variable (positive affect or activity pleasantness, respectively). $\mathrm{MBCT}=$ Mindfulness-Based Cognitive Therapy; $\mathrm{CONTROL}=$ waitlist control condition.

$* \mathrm{p}<.05 ; * * \mathrm{p}<.01 ; * * * \mathrm{p}<.001$ 

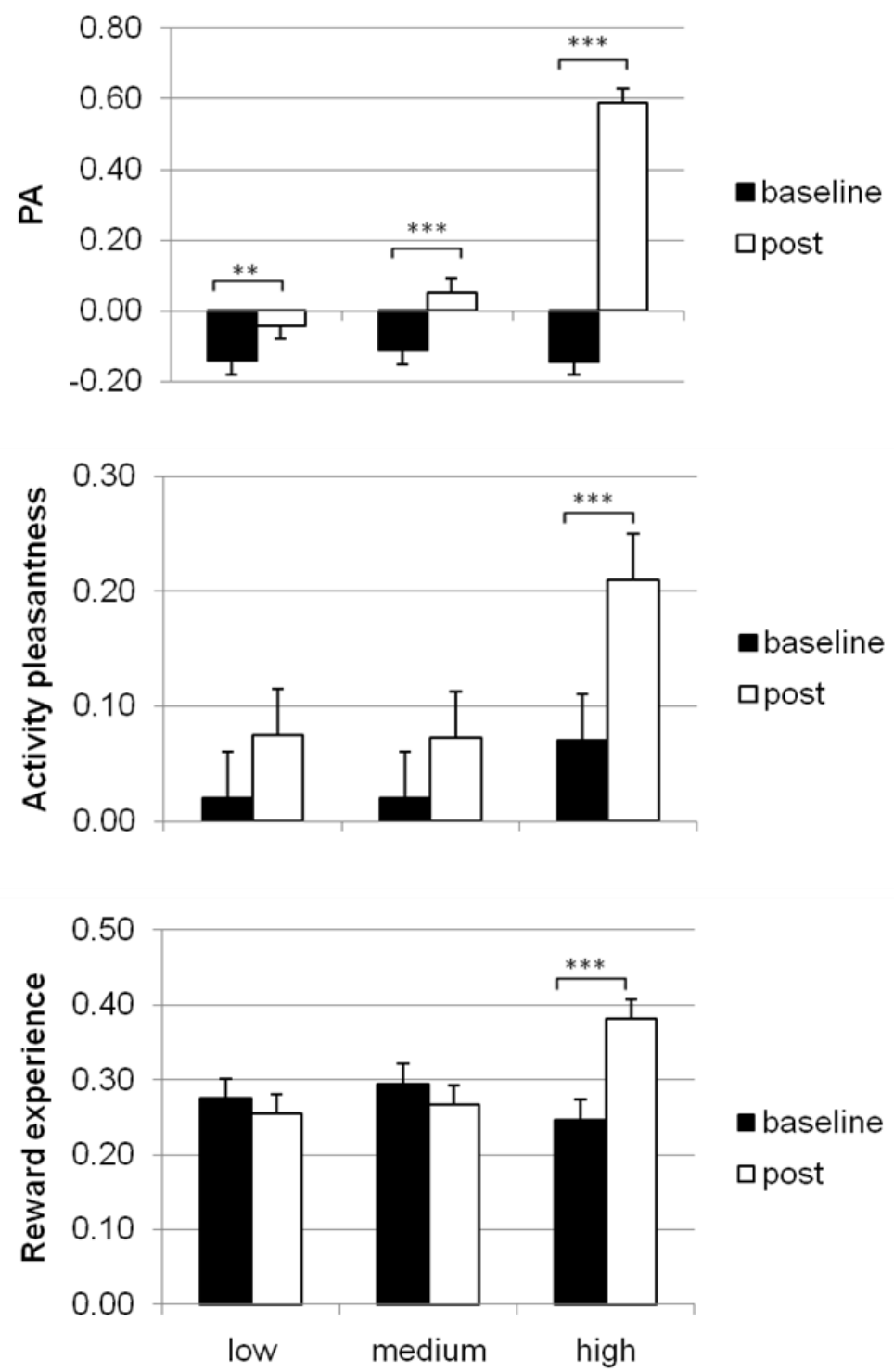

Figure 3. Association between reduction of depressive symptoms and positive affect, activity pleasantness, and reward experience, before and after MBCT. Analyses were controlled for a person's mean baseline level on the dependent variable (positive affect or activity pleasantness, respectively). Bars represent standardized predicted values ( $\pm \mathrm{SE}$ ) in low, medium, and high responders (MBCT group only). Differences between baseline and post-assessment increased with clinical response. HDRS = Hamilton Depression Rating Scale; MBCT = Mindfulness-Based Cognitive Therapy.

$* p<.05 ; * * p<.01 ; * * * p<.001$ 


\section{DISCUSSION}

\section{Current findings}

Effects of Mindfulness-Based Cognitive Therapy (MBCT) were compared to a waitlist control condition (CONTROL) in adults with a history of major depression and current residual symptoms. Overall, our results support the primary hypothesis that $\mathrm{MBCT}$ is associated with both enhanced experience of pleasant daily-life situations and improved positive affect (PA) responsiveness to pleasant daily-life situations. Results showed that MBCT was associated with (a) more overall PA, (b) higher appraisal of activities as pleasant, and (c) higher levels of reward experience, compared to baseline, and compared to CONTROL. Special attention was given to the question whether PA-related changes after MBCT may have simply been epiphenomena of reduced depression. The effects of treatment condition remained similar in analyses additionally correcting for reduction of depressive symptoms, indicating that MBCT affected changes in PA-related variables independent of degree of improvement in depressive symptomatology. Further analyses examined to which extent effects of MBCT on PA related variables were independent of changes in worry, rumination (variables on which $M B C T$ research has focused so far), and negative affect. Effect sizes of MBCT on PA-related variables were reduced but remained significant when changes in worrying, rumination, and negative affect were included.

In line with our secondary hypothesis, increases in positive affect variables within the MBCT group were associated with reductions in residual depressive symptoms, indicating the potential relevance of increased positive affect for the prevention of depression. There was a dose-response relation between reductions on the clinician-rated Hamilton Depression Rating Scale (HDRS) and the PA-related variables. MBCT participants with the highest reduction in residual symptoms (based on a tertile split of the distribution) experienced significantly stronger increases in PA, activity pleasantness, and reward experience than those with medium improvement, who in turn experienced stronger increases that those in the low improvement group (except for activity pleasantness and reward experience, where only the high improvement group differed from the other two groups).

\section{The other face of MBCT: changing the experience of positive emotions}

Our findings suggest that modification of a resilience phenotype (more frequent experience of daily-life PA, as well as enhanced PA responsiveness to pleasant daily-life activities) is possible. Increases in PA, activity appraisal, and reward 
experience were specific to $\mathrm{MBCT}$ (with no significant increases in the control condition), and partially independent of more commonly observed decreases in worry, rumination and negative affect (Raes, et al., 2009; Teasdale, et al., 1995; Teasdale, et al., 2000). This points to the possibility that MBCT may directly facilitate the experience of positive emotions, as suggested by Garland and colleagues (2010). For example, enhanced engagement with the present experience (one of the main goals of $\mathrm{MBCT}$ ) is associated with broadening of attention (Lutz, Slagter, Dunne, \& Davidson, 2008), and may therefore lead to higher awareness of pleasant situations (which are more easily overlooked than unpleasant situations; Baumeister, et al., 2001). This higher awareness of pleasant situations, in combination with the curious and mild attitude taught during MBCT, may in turn lead to increases in individuals' hedonic capacity (Schroevers \& Brandsma, 2010).

Although the current study provides initial support for the assumption that MBCT may increase the experience of reward and positive emotions (phenotypes that have been associated with better resilience against depressive symptomatology), future research should examine whether or not the experimental modification of reward experience represents a mechanism of change of MBCT. Kazdin (2007) provides a compelling account about how research on mindfulness mechanisms may best progress. For example, an association between the extent of the experimental increase in reward experience and a more favorable future course of illness would support the hypothesis of reward experience as a mechanism of change of MBCT. Studies might also investigate whether increased engagement with the present experience (as proposed above), or rather changes in third variables drive greater appreciation of, and enhanced responsiveness to pleasant activities. Furthermore, without an active treatment comparison it is impossible to know whether the changes in PA are specifically due to increased mindfulness. Therefore, future studies investigating associations between $\mathrm{MBCT}$ and change in PA should include a third attention-control arm (or alternatively a 'pure' arm, in which nourishing or rewarding activities are not at all addressed during the intervention). Finally, research investigating whether certain individual differences or clinical characteristics determine individual variation in the primary changes after MBCT would also be valuable. For example, some participants may benefit from MBCT mainly through reduction of worrying, while others may benefit mainly through changes in the experience of positive emotions. 


\section{Clinical Implications}

A growing body of literature indicates that the efficient generation of positive emotions may be a resilience phenotype: PA is very important for well-being in general (Folkman \& Moskowitz, 2000; Seligman, et al., 2005) and the prevention of and recovery from depression in particular (Garland, et al., 2010; Wichers, et al., 2009a). Several studies suggest that it would be clinically relevant to change the ability to experience of PA if that were possible. High levels of reward experience have been shown to protect against the development of affective symptoms (Geschwind, et al., 2010; Wichers, et al., 2010), and were associated with recovery from depression after pharmacotherapy (Wichers, et al., 2009a) in other, unrelated samples. The present study, to our knowledge, is the first to show that a behavioral, non-pharmacological intervention is associated with enhanced generation of PA during pleasant daily-life activities. However, due to the lack of an active intervention group, we cannot assume causality. An alternative explanation for our findings is that decreases in depressive symptoms could lead to increases in positive affect.

Despite statistical significance, the clinical significance may seem less obvious, because increases in PA are relatively small (e.g., PA increased from 3.7 to 4.2 on a 7-point Likert scale in the MBCT condition). On the other hand, a previous study showed that very small increases in PA can be strong predictors of clinically relevant change. For example, small improvements in daily-life PA (from 3.3 to 3.6 on a 7-point Likert scale) during the first week of antidepressant treatment were associated with a 34 times higher chance on achieving remission six weeks later, compared to no change or worsening of PA during the first week (Geschwind et al, 2010a).

Another clinically relevant finding is that increased reward experience following MBCT co-occurred with the reduction of residual complaints (which are harmful and increase the risk for future episodes of major depression; Judd, et al., 1998). Effects of MBCT on PA-related variables did not change when corrected for change in residual depressive symptoms, indicating that PA-related improvements were not simply epiphenomena of reductions in depressive symptoms. To verify this claim more definitely, however, replication of results in a symptom-free sample is desirable. Furthermore, future studies should look beyond changes in traditionally researched $\mathrm{MBCT}$ outcome variables and investigate to what extent MBCT-induced changes in positive emotionality and other resilience-related constructs contribute to recovery from depression and relapse prevention. 


\section{Strengths and limitations}

Strengths of the current study include the use of the Experience Sampling Method (ESM) to measure changes in positive affectivity. ESM repeatedly measures positive emotions as they occur in daily life, thus increasing ecological validity and minimizing memory bias (Csikszentmihalyi \& Larson, 1987). Although ESM relies on self-report, demand bias for the main outcome (change in PA-related variables) was unlikely for the following reasons: (i) positive affect items were hidden among other items describing the current activity and situation, and participants were blind to the hypotheses; (ii) MBCT's main focus is to encourage participants to engage with the present moment (although MBCT does also address nourishing activities), and (iii) most previous research has focused on negative emotions, rumination, or worry. Another strength of the current study is that inclusion criteria were intentionally kept at a low threshold in order to enhance generalizability. Moreover, attrition rate was low, and the main analyses focused on the intention-to-treat sample, thus analyzing all participants randomized to treatment. Finally, the study was a randomized controlled trial, thus reducing selection bias, and there were no prior differences in baseline or clinical characteristics between MBCT and CONTROL.

Limitations are (a) that the current trial did not include an active control intervention arm, making conclusions about active treatment ingredients impossible, (b) the all-Caucasian sample, and (c) the absence of objective information on competent treatment delivery and adherence to the MBCT protocol. Another limitation is (d) the reliance on self-reported entry completion time for distinguishing valid from invalid experience sampling reports. However, an earlier study comparing self-reported completion time and objective completion time (obtained through monitoring by hidden electronic devices; Jacobs, et al., 2005) indicates that self-reported completion times are reliable. Furthermore, (e) both participants and assessors were not blind to treatment condition due to pragmatic limitations. Importantly though, participants and assessors were unaware of direction and extent of change in PA-related variables (the main trial outcome) when assessing residual depressive symptoms. Also, associations between increases in PA-related variables and decreases in residual depressive symptoms were unlikely to be due to biased assessment, because results of self-report (IDS-SR) and interview-based (HDRS) assessment of residual depressive symptoms corresponded. A further limitation (f) is that it is impossible to say whether increase in activity appraisal indeed represents a tendency to appraise similar activities as more pleasant than before MBCT or rather a tendency to engage in more pleasant activities. However, either option has clinical 
relevance. The choice not to have external observers rate pleasantness of activities was made consciously, because the experience of every-day activities is entirely subjective. Moreover, this was a within-subject design and participants were compared to themselves. Finally, (g) the low-threshold inclusion criteria generate more heterogeneity between participants. However, there were no significant differences on any key variables between MBCT and CONTROL groups at baseline. Furthermore, the low-threshold inclusion criteria warrant generalizability to most individuals with residual depressive symptoms (though replication in a symptom-free sample would be desirable).

\section{Conclusion}

This is the first study to show that a behavioral intervention can lead to increased experience of momentary positive emotions, greater appreciation of, and enhanced responsiveness to pleasant daily-life activities. From a clinical point of view, this is a very relevant finding because it means that a non-pharmacological intervention may potentially improve the reward system. The clinical impact is considerable, because it makes prevention efforts targeting reward experience in vulnerable groups more acceptable.

More research is needed with regard to active treatment ingredients, mechanisms of change, and the degree to which positive emotions and other resilience-related phenotypes contribute to recovery from depression and prevention of relapse or recurrence.

\section{Acknowledgements}

Marieke Wichers was supported by the Dutch Organisation for Scientific Research (NWO, VENI Grant Nr. 916.76.147). We thank our study participants for their time and effort. Furthermore, we thank Rufa Diederen for her help with data collection, and Truda Driesen, Philippe Delespaul, Frieda van Goethem, and the 4D database team for their help with data entry. 


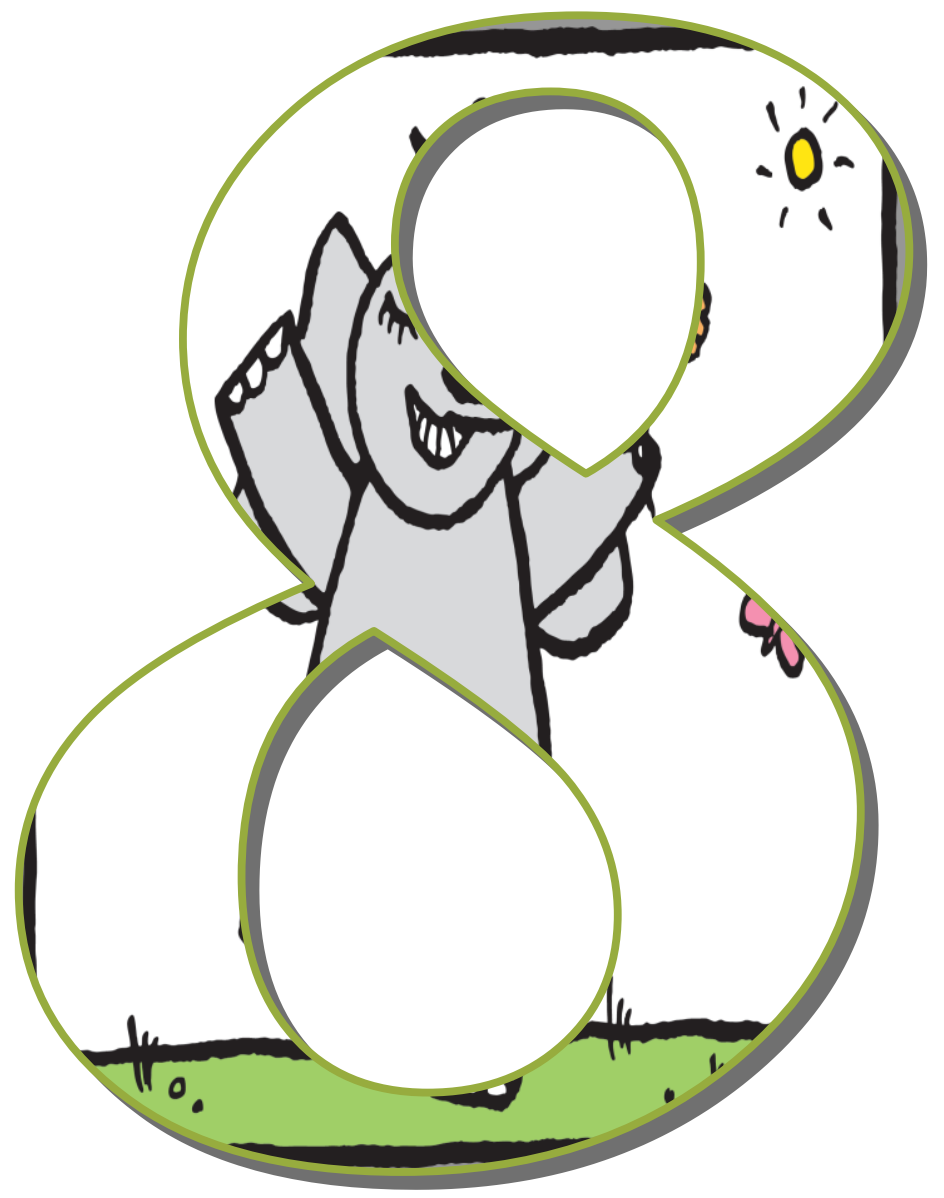

Mindfulness-Based Cognitive Therapy Reduces Residual Depressive Symptoms Irrespective of Number of Prior Depressive Episodes. A Randomized Controlled Trial 
Objective: To investigate whether exclusion of patients with only one or two prior episodes of major depression from most Mindfulness-Based Cognitive Therapy (MBCT) trials (based on findings of differential subgroup efficacy; Ma \& Teasdale, 2004; Teasdale, et al., 2000) is justified.

Method: Adults with a life-time history of depression and current residual depressive symptoms (mean age $=43.9$ years, $S D=9.6 ; 75 \%$ female; all Caucasian) were randomized to MBCT $(n=64)$ or waitlist control (CONTROL; $n=$ $66)$ in a parallel, open-label, randomized controlled trial. Randomization occurred within subgroups: $\leq 2$ prior episodes $(n=71)$ vs. $\geq 3(n=59)$. Primary outcome measure was reduction in residual depressive symptoms, measured using Hamilton Depression Rating Scale (HDRS-17) and Inventory of Depressive Symptoms (IDS).

Results: The interaction between treatment and subgroup was not significant ( $p>$ .2). MBCT was superior to CONTROL across subgroups $(p<.001)$. Effect sizes of MBCT compared to CONTROL were nonsignificantly larger in participants with $\leq 2$ than in participants with $\geq 3$ prior episodes (HDRS: $d=-.74$ vs. -.29 ; IDS: $d=-.60$ vs. -.26, respectively).

Conclusions: In a sample of participants with residual depressive symptoms, we found no evidence for increased efficacy of MBCT in participants with $\geq 3$ compared to $\leq 2$ prior episodes, challenging the recent practice of excluding patients with less than three prior episodes from MBCT trials and treatment. Presence of residual symptoms, rather than number of prior episodes, may determine efficacy of MBCT.

Keywords: residual symptoms; major depression; behavioural treatment; relapse; recurrent affective disorder

Published as:

Geschwind, N., Peeters, F., Van Os, J., \& Wichers, M. (submitted). Mindfulnessbased cognitive therapy reduces residual depressive symptoms irrespective of number of prior depressive episodes. A randomized controlled trial. 
Mindfulness-Based Cognitive Therapy (MBCT) is an effective group skills-training program specifically designed to prevent relapse of depression. MBCT combines methods of mindfulness training (Kabat-Zinn, 1990) with features of cognitive therapy for depression (Beck, et al., 1979; Teasdale, et al., 1995). The original idea behind $\mathrm{MBCT}$ was to train participants to fully experience the present moment and disengage from automatic negative thinking patterns that arise during dysphoric mood and facilitate relapse (Teasdale, et al., 2000). Several studies have shown that $\mathrm{MBCT}$ is effective in reducing depressive symptoms and relapse risk (Bondolfi, et al., 2010; Kuyken, et al., 2008; Ma \& Teasdale, 2004; Teasdale, et al., 2000). In addition, recent studies confirm that MBCT reduces cognitive reactivity (Kuyken, et al., 2010; Raes, et al., 2009), as well as rumination and worrying (Evans, et al., 2008; Geschwind, Peeters, Drukker, Van Os, \& Wichers, 2011b; Williams, 2008).

\section{DIFFERENTIAL EFFECTIVITY}

After the initial study by Teasdale and colleagues (2000) on differential risk of relapse associated with $\mathrm{MBCT}$, and $\mathrm{Ma}$ and Teasdale's subsequent replication (2004), Teasdale and coworkers postulated that MBCT was effective for prevention of relapse only in patients with three or more episodes of depression (hereafter referred to as $3+$ ). Based on these findings, patients with fewer than three episodes of depression (hereafter referred to as 2-) were often excluded a priori in studies examining the effectiveness of MBCT in depression (Bondolfi, et al., 2010; Godfrin \& van Heeringen, 2010; Kuyken, et al., 2008; Kuyken, et al., 2010; Segal, et al., 2010). Even when studies did not directly examine relapse prevention but related aspects such as residual depressive symptoms, researchers frequently restricted samples to patients with three or more depressive episodes (Barnhofer, et al., 2009; Hargus, Crane, Barnhofer, \& Williams, 2010; Kingston, Dooley, Bates, Lawlor, \& Malone, 2007). However, the evidence for excluding 2patients is not indisputable, as stated by Ma and Teasdale (2004) themselves. The overlap and diversity arguments discussed below support the notion that further research on this topic is necessary.

\section{THE OVERLAP ARGUMENT}

Recommendations for excluding 2- patients were based on only two studies from the same country and research group (the original developers of MBCT; Ma \& Teasdale, 2004; Teasdale, et al., 2000). In both studies, patients in the 2- group 
had experienced significantly more stressful life events before relapsing than patients in the 3+ group. Patients therefore may have been assessed at different stages of their lives, or they may have belonged to different subpopulations, as $\mathrm{Ma}$ and Teasdale (2004) suggest. However, it can be postulated that there should be some degree of overlap between these groups, given that all patients in the 3+ group once belonged to the 2- group, and part of the 2- group will transgress to the $3+$ group over time. Provided participants learn a set of skills which they can afterwards apply when necessary, MBCT should prevent or delay transgression to the $3+$ group in at least a subsample of 2 - patients.

\section{THE DIVERSITY ARGUMENT}

The rationale explaining why MBCT may be more effective for patients with three or more depressive episodes is based on the assumption that MBCT works mainly on cognitive processes (reduction of rumination and changes in meta-cognition). By encouraging disengagement from rumination and reducing cognitive reactivity to sad mood, as indeed shown in several studies (Kumar, Feldman, \& Hayes, 2008; Kuyken, et al., 2010; Raes, et al., 2009; Ramel, Goldin, Carmona, \& McQuaid, 2004), MBCT may prevent episodes elicited mainly by the downward effects of being caught up in negative thinking spirals. From this point of view, it makes sense that MBCT may be more effective in 3+ patients, given that research has shown that, compared to earlier depressive episodes, subsequent episodes are increasingly independent of stressful life events and more strongly associated with internal cognitive processes (Kendler, et al., 2000; Monroe \& Harkness, 2005; Post, 1992; Segal, et al., 1996).

However, evidence indicates that MBCT not only improves dysfunctional cognitive processes but also leads to independent changes in emotional experience. Fredrickson and colleagues report increased levels of positive emotions in working adults after an eight-week meditation training, compared to a waiting list control group. Changes in positive emotions in turn predicted increased personal resources, increased life satisfaction, and reduced depressive symptoms (Fredrickson, et al., 2008). In another randomized controlled trial, mindfulness meditation was associated with increased left-sided anterior activation (Davidson, et al., 2003), a pattern consistent with increased positive affect (Davidson, 1992; Davidson, Ekman, Saron, Senulis, \& Friesen, 1990). A recent report on the current randomized controlled trial also found increased levels of positive emotions and decreased levels of negative emotions after MBCT in participants with residual depressive symptoms, compared to CONTROL. Again, 
increases in positive emotions were associated with decreases in residual depressive symptoms (independently of changes in cognitive variables; Geschwind, et al., 2011). A review on advances in affective neuroscience concludes that positive emotions induce upward spirals, which can effectively counter downward depressive spirals (Garland, et al., 2010). There is evidence that this process may occur independently of cognitive change (Geschwind, et al., 2011b; Tang, Luborsky, \& Andrusyna, 2002). Garland and colleagues' review (2010) furthermore highlights MBCT as an intervention supporting the generation of positive emotions. Therefore, although MBCT leads to improvements in metacognition, it also appears to work on a different level.

Why improvements in emotional experience and other health-related variables (Baer, 2006; Davidson, et al., 2003) would remain restricted to 3+ patients, however, remains obscure, particularly given that mindfulness and other meditation-based interventions are beneficial in various patient populations without major depression, as well as in community samples (Baer, 2003, 2006; Evans, et al., 2008; Fredrickson, et al., 2008; Kabat-Zinn, 2003). It may be argued, therefore, that if $\mathrm{MBCT}$ contributes to health and well-being in individuals who never suffered from depression, there is no strong case for the expectation that beneficial effects will be limited to $3+$ participants. If this were the case a ushaped function would result, with MBCT being beneficial for participants with high levels of distress but no formal diagnosis of depression, as well as for 3+ participants, but not 2-participants.

The above-mentioned arguments support the need for further research on the question whether MBCT is indicated only for patients with more than three prior episodes of depression. However, further research must not necessarily use time to relapse as an outcome measure. Improvement in residual symptoms may also be a valid and useful outcome, as reasoned below.

\section{RESIDUAL DEPRESSIVE SYMPTOMS AS OUTCOME MEASURE?}

The outcome variable (time to relapse) in the studies by Teasdale and colleagues is based on a dichotomous classification (relapse vs. no relapse). Given mounting evidence for continuity of disease expression as well as problems associated with dichotomous classification in healthcare (e.g., arbitrary symptom clusters and thresholds; see Kendler \& Gardner, 1998), it may be useful to additionally examine continuous measures, for example residual depressive symptoms. The course of unipolar depression is characterized by waxing and waning of symptoms in many patients (Judd, et al., 2002), and the degree to which patients continue to 
experience residual symptoms of depression is directly related to risk for relapse (Judd, Paulus, \& Zeller, 1999; Nierenberg, et al., 2010). Studies have already shown that MBCT is associated with reduction of depressive symptoms in the short- and long-term (Kenny \& Williams, 2007; Kingston, et al., 2007; Mathew, Whitford, Kenny, \& Denson, 2010). However, these studies were either small (Kingston, et al., 2007), observational and non-randomized (Mathew, et al., 2010), or examined acutely depressed, treatment-resistant patients (Kenny \& Williams, 2007). Moreover, none of these studies examined differences between 2- and 3+ patients.

A second reason why it may be important to focus on residual symptoms is that these may potentially confound the impact of number of prior episodes, as latest findings suggest. Segal and colleagues recently investigated the role of stable versus unstable remission in the effectiveness of $\mathrm{MBCT}$ for relapse prevention in 3+ patients (Segal, et al., 2010). Among unstable remitters (i.e., those who experienced residual depressive symptoms during remission), MBCT was superior to placebo ( $74 \%$ decrease in risk for relapse) and equally effective as continuation with active antidepressants. Among stable remitters (i.e., those who did not experience residual symptoms during remission), there was no difference between continuation with $\mathrm{MBCT}$, active antidepressants, or placebo. This study thus highlights the importance of residual symptoms and suggests the need to further investigate the effectiveness of MBCT in 2- patients with residual depressive symptomatology. This issue is particularly relevant given that patients with residual symptoms (scores of ten or more on the Hamilton Depression Rating Scale) were actually excluded in the initial studies on differential relapse prevention (Ma \& Teasdale, 2004; Teasdale, et al., 2000).

\section{HYPOTHESES}

The aim of the current study is to examine the effectiveness of MBCT in participants with residual symptoms of depression after either one or two versus three or more prior episodes of major depression. The main outcome measure is reduction in residual depressive symptoms. Given the arguments outlined above, we expect that a) $\mathrm{MBCT}$, compared to the waiting list control condition (CONTROL), will be associated with reduction of residual symptoms, and that b) reductions will be independent of the number of prior episodes of major depression. Hypotheses are tested in an open-label, parallel, randomized controlled trial comparing participants who continued with treatment as usual to participants who, additionally, received eight weeks of MBCT. Participants were 
stratified according to the number of episodes of major depression (two or fewer vs. three or more) prior to randomization. In order elucidate possible different mechanisms of improvement in the two subgroups, we also explored whether the number of prior episodes of major depression was associated with differential changes in other variables of interest, such as positive and negative emotions, rumination, worry, and mindfulness skills.

\section{METHOD}

\section{Participant Characteristics}

For the current study (acronym MindMaastricht; trial number NTR1084, Netherlands Trial Register), adults with residual symptomatology after at least one episode of Major Depressive Disorder were recruited from outpatient mental health care facilities in Maastricht and through posters in public spaces. Residual symptoms are associated with higher risk of relapse (Judd, et al., 1998) and were therefore required as an indicator of vulnerability to depression. Residual symptoms were defined as a score of seven or higher on the 17-item Hamilton Depression Rating Scale (HDRS; Hamilton, 1960) at the time of screening. Exclusion criteria were: fulfilling criteria for a current depressive episode, schizophrenia, psychotic episodes in the past year, and recent (past four weeks) or upcoming changes in ongoing psychological or pharmacological treatment. Currently depressed individuals were excluded because, at trial preparation, there was no evidence that currently depressed individuals were able to participate in or benefit from MBCT. Relevant sociodemographic and clinical characteristics are displayed in Table 1.

\section{Sampling Procedures}

All study procedures were approved by the standing Medical Ethics Committee of Maastricht University Medical Centre, and all participants signed an informed consent form. An initial screening of potential participants was performed by telephone to check for availability during the study period and likelihood of meeting in- and exclusion criteria. A second screening included administration of the Structured Clinical Interview for DSM IV axis I (SCID-I; First, et al., 2002) and the 17-item HDRS by trained psychologists. Eligible participants were invited for a detailed one-on-one explanation of the study procedures, and then took part in the baseline assessment. The baseline assessment consisted of six days of experience sampling (ESM) in participants' daily-life environment, and subsequent administration of a battery of questionnaires (see section measures) as well as the HDRS interview (in the laboratory). ESM is a momentary assessment technique 
designed to measure affect in the flow of daily life (which was the focus of a previous report on the same sample; Geschwind, et al., 2011). After the baseline assessment, participants were randomized to either MBCT or CONTROL (allocation ratio 1:1) if they were likely to have at least 20 valid ESM assessments (see Delespaul, 1995; Geschwind, et al., 2011b). After either eight weeks of MBCT (see section "Intervention") or equivalent waiting time (in the CONTROL condition), participants again took part in six days of experience sampling, followed by administration of the HDRS and questionnaires. All participants were compensated with gift vouchers worth 50 Euros. Participants in the CONTROL condition were offered the opportunity to take part in MBCT after the postintervention assessment. Participant flow is displayed in Figure 1.

Randomization was stratified according to number of depressive episodes (two or fewer versus three or more), as previous studies suggest a greater benefit for those with three or more previous episodes (Ma \& Teasdale, 2004; Teasdale, et al., 2000). An independent researcher not involved in the project generated the randomization sequence in blocks of five (using the sequence generator on www.random.org), and wrote the randomization code in sealed numbered envelopes. After completion of all baseline assessments, the researcher allocated participants to their treatment condition based on the randomization code in the sealed envelope (opened in order of sequence). No masking of treatment condition took place.

\section{Sample Size and Power}

Sample size $(n \geq 120)$ was determined on the basis of sufficient power for geneenvironment interactions (not analyzed here). A power analysis to detect a significant interaction for a linear contrast of means was conducted by assuming that the difference in the mean reduction of the total HAM-D score between the MBCT and CONTROL conditions would be 2 points higher in the $3+$ group compared to the 2- group. Given a standard deviation of 3.4 in HAM-D change scores in a different sample and a total sample size of 130, approximately evenly split among the four groups, we obtain a power of just under $40 \%$ to detect a significant interaction at $\alpha=.05$.

\section{Intervention (MBCT)}

Content of MBCT trainings sessions followed the protocol of Segal et al. (2002). Trainings consisted of eight weekly meetings lasting $2.5 \mathrm{hr}$ and were run in groups of 10-15 participants (thus occasionally larger than the usual 10-12 participants per group). Assessment periods for control participants were matched to those of MBCT participants. Sessions included guided meditation, experiential exercises, 
and discussions. In addition to the weekly group sessions, participants received CDs with guided exercises and were assigned daily homework exercises (30-60 min daily). Trainings were given by experienced trainers in a center specialized in mindfulness trainings. All trainers were supervised by an experienced health care professional who had trained with Teasdale and Williams, the co-developers of MBCT (Segal, et al., 2002).

\section{Measures}

Hamilton Depression Rating Scale (HDRS).

The 17-item HDRS was administered by two trained research assistants with Master degrees in Psychology. The HDRS (Hamilton, 1960) is a semi-structured interview designed to assess depressive symptoms over the past week. It is one of the most often used rating scales in depression research, and internal, interrater, and retest reliability estimates for the overall HDRS are good (Bagby, et al., 2004). Only the overall score was used for the analyses, and interrater reliability for the total score was high (IntraClass Correlation coefficient $=0.97$ ). To provide information on interrater reliability, both interviewers had independently rated eight videotaped HDRS interviews with patients varying in strength of residual depressive symptoms.

Inventory of Depressive Symptoms (Self-Rating; IDS-SR).

The IDS-SR (Rush, et al., 1996) is a self-rated scale, which includes 30 items rated 0 to 3. Because the rating of "appetite" and "weight" is duplicated (separate items for increases and decreases), only 28 items are taken into account for the final score. The scale is sensitive to change and has good psychometric properties (Rush, et al., 1996). Internal consistency in the current sample was .85.

Penn State Worry Questionnaire (PSWQ)

A Dutch version of the 16-item PSWQ was used to assess worrying. The PSWQ emerged from factor analysis of a large number of items and was found to possess high internal consistency and good test-retest reliability (Meyer, et al., 1990). Internal consistency in the current sample was .90.

Rumination on Sadness Scale (RSS).

The approved Dutch backtranslation of the RSS was used (Raes, et al., 2003) to measure rumination. The RSS contains 13 items comprising one factor, has an adequate test-retest stability over a 2- to 3-week period, and has good convergent and discriminant validity (Conway, et al., 2000). Internal consistency in the current sample was .90, similar to earlier studies (e.g., Conway, et al., 2000). 
Kentucky Inventory of Mindfulness Skills (KIMS).

The approved and validated Dutch version (Dekeyser, Raes, Leijssen, Leysen, \& Dewulf, 2008) of the 39-item KIMS was used to measure mindfulness skills. The KIMS was chosen as a measure of mindfulness skills because it is suitable for use by novices. Moreover, its subscales are sensitive to change and have been validated also in clinical samples (Baer, Smith, \& Allen, 2004; Baum, et al., 2010). The KIMS has four subscales: Observe, Describe, Act With Awareness and Accept Without Judgement. Internal consistency of the subscales in the current sample was .84, .91, .81, and .88, respectively.

\section{$P A$ and NA}

At each beep, several ESM mood adjectives were assessed on 7-point Likert scales ranging from 1 (not at all) to 7 (very). Consistent with previous work (MyinGermeys, et al., 2001; Wichers, et al., 2010), principal component factor analysis with oblique rotation was used to generate a factor representing PA and a factor representing NA. The mood adjectives "happy", "satisfied", "strong", "enthusiastic", "curious", "animated", and "inspired" loaded on the PA factor ( $\alpha=$ .89), whereas "down", "anxious", "lonely", "suspicious", "disappointed", "insecure", and "guilty" loaded on the NA factor $(\alpha=.86)$. One mood item ("I feel relaxed") was not included in the PA factor due to low factor loadings (<.6). Mean levels of PA and NA were then computed per participant and beep moment.

\section{Statistical Methods}

Analysis was intention-to-treat. Primary outcome measures were residual depressive symptoms expressed as total HDRS and IDS-SR scores. Secondary outcome measures were total scores of worry (PSWQ), rumination (RSS), positive and negative affect (experience sampling method), and subscale scores of mindfulness skills (KIMS). Linear regression analyses were used to examine the effect of the interaction between treatment condition (MBCT vs. CONTROL) and number of previous depressive episodes (2-vs. $3+$ ) on the (post-measurement) outcome, always corrected for baseline values of that measure. Additional analyses checked whether results differed when variables with significant between-group differences were included. These results were only reported in detail when dissimilar. For positive and negative emotions, which were measured repeatedly per measurement occasion, a multi-level approach was used (see Wichers, et al., 2009). The statistical package Stata 11 was used for all analyses (StataCorp, 2009). Standardized effect sizes d (interpretable according to Cohen's criteria; Cohen, 1992) are reported whenever results concern effect sizes. Tables and figures of means display unstandardized values. 
Despite the low power to detect an interaction, statistical tests for interaction are recommended as the most appropriate statistical method for making inferences from subgroup analyses, because they recognize the limited extent of data available and therefore inhibit potentially premature claims of subgroup findings (Pocock, Assmann, Enos, \& Kasten, 2002). Nevertheless, for comparability with prior research, and in light of the low power for detecting an interaction, effect sizes of MBCT relative to CONTROL are also presented within subgroups.

\section{RESULTS}

\section{Participants}

Recruitment started in January 2008 and ended in February 2009, and all postintervention assessments were completed by August 2009, when the predetermined number of participants was reached. At baseline, there were no large or significant differences between subgroups with respect to sociodemographic and clinical characteristics, except for the distribution of gender (see Table 1). Table 2 shows baseline and post assessment scores of primary and secondary outcome measures by subgroup. Only IDS-SR scores differed significantly between groups at baseline, otherwise there were no large or significant baseline differences between groups. Participant flow through the study is displayed in Figure 1 . Note that one participant only completed the HDRS but not the other measures at the post measurement occasion. No known harms or unintended treatment effects were reported in either group. On average, participants randomized to $\mathrm{MBCT}$ attended 7.2 sessions $(\mathrm{SD}=1.5$ ), with no significant differences between the 2 - and $3+$ groups. Three MBCT participants attended less than 3 sessions (two 2- participants and one $3+$ participant). There was no difference in daily mindfulness practice between the 2- and 3+ MBCT group: 2participants in the MBCT group reported having practiced for 28 minutes $(S D=13$ ) per day on average, and 3+ participants reported a daily average of 32 minutes $(S D=13)$. There were no baseline differences with regard to mindfulness skills, as shown in Table 2. 
Table 1

Baseline demographic and clinical characteristics by treatment condition and number of prior major depressive episodes.

\begin{tabular}{|c|c|c|c|c|}
\hline \multirow[b]{2}{*}{ Measure } & \multicolumn{2}{|c|}{2 or less prior episodes } & \multicolumn{2}{|c|}{3 or more prior episodes } \\
\hline & $\begin{array}{l}\text { CONTROL } \\
(n=36)\end{array}$ & $\begin{array}{l}\text { MBCT } \\
(n=35)\end{array}$ & $\begin{array}{l}\text { CONTROL } \\
(n=30)\end{array}$ & $\begin{array}{l}\text { MBCT } \\
(n=29)\end{array}$ \\
\hline Age (mean, SD) & $43.1(8.8)$ & $42.5(10.6)$ & $43.4(10.4)$ & $47.1(8.0)$ \\
\hline Female gender ${ }^{a}$ & $58 \%$ & $83 \%$ & $90 \%$ & $72 \%$ \\
\hline Full-/part-time work & $75 \%$ & $74 \%$ & $60 \%$ & $48 \%$ \\
\hline $\begin{array}{l}\text { Illness/unemployment } \\
\text { benefits }\end{array}$ & $22 \%$ & $14 \%$ & $23 \%$ & $28 \%$ \\
\hline $\begin{array}{l}\text { Living with partner/own } \\
\text { family }\end{array}$ & $59 \%$ & $66 \%$ & $57 \%$ & $59 \%$ \\
\hline $\begin{array}{l}\text { Comorbid anxiety disorder } \\
\text { (present) }\end{array}$ & $42 \%$ & $29 \%$ & $57 \%$ & $41 \%$ \\
\hline $\begin{array}{l}\text { Comorbid anxiety disorder } \\
\text { (past) }\end{array}$ & $56 \%$ & $43 \%$ & $73 \%$ & $62 \%$ \\
\hline $\begin{array}{l}\text { Current psycho- } \\
\text { counseling/-therapy }\end{array}$ & $11 \%$ & $9 \%$ & $13 \%$ & $21 \%$ \\
\hline $\begin{array}{l}\text { Current use of } \\
\text { antidepressants }\end{array}$ & $32 \%$ & $27 \%$ & $47 \%$ & $39 \%$ \\
\hline $\begin{array}{l}\text { (Occasional) use of } \\
\text { benzodiazepines }\end{array}$ & $9 \%$ & $3 \%$ & $7 \%$ & $14 \%$ \\
\hline
\end{tabular}

Note. $\mathrm{MBCT}=$ Mindfulness-Based Cognitive Therapy; $\mathrm{CONTROL}$ = waitlist control condition .

${ }^{a}$ Apart from gender $(p=.02)$, there were no significant interactions between treatment condition and number of episodes (at $p<.05)$. 
Table 2

Means (SD) of primary and secondary outcome measures by treatment condition, measurement occasion, and number of prior episodes.

\begin{tabular}{|c|c|c|c|c|}
\hline \multirow[b]{2}{*}{ Measure } & \multicolumn{2}{|c|}{ CONTROL } & \multicolumn{2}{|c|}{ MBCT } \\
\hline & pre & post & pre & post \\
\hline & \multicolumn{4}{|c|}{2 or less prior episodes } \\
\hline HDRS & $10.5(3.7)$ & $9.8(4.1)$ & $9.6(3.2)$ & $5.9(4.2)$ \\
\hline IDS-SR & $23.8(8.8)$ & $20.3(9.9)$ & $19.3(9.4)$ & $11.4(8.9)$ \\
\hline RSS & $41.0(9.6)$ & $39.1(9.7)$ & $41.1(9.6)$ & $33.1(9.8)$ \\
\hline PSWQ & $59.8(11.2)$ & $57.3(10.7)$ & $58.9(10.3)$ & $49.7(11.7)$ \\
\hline KIMS observe & $40.9(7.0)$ & $40.7(7.2)$ & $39.5(7.0)$ & $43.9(6.0)$ \\
\hline KIMS describe & $26.6(6.4)$ & $27.4(7.0)$ & $27.3(7.0)$ & $28.6(6.4)$ \\
\hline KIMS act aware & $25.9(5.1)$ & $26.8(4.5)$ & $26.3(4.8)$ & $31.2(5.2)$ \\
\hline KIMS accept & $28.7(7.1)$ & $30.0(6.8)$ & $27.4(6.7)$ & $31.7(7.3)$ \\
\hline PA & $4.0(.9)$ & $3.9(1.0)$ & $3.9(.7)$ & $4.3(.9)$ \\
\hline \multirow[t]{2}{*}{ NA } & $2.1(.8)$ & $2.2(.9)$ & $1.9(.7)$ & $1.5(.6)$ \\
\hline & \multicolumn{4}{|c|}{3 or more prior episodes } \\
\hline HDRS & $9.9(3.4)$ & $9.5(4.0)$ & $11.1(4.1)$ & $8.6(5.1)$ \\
\hline IDS-SR & $20.5(8.2)$ & $17.9(9.1)$ & $26.0(11.1)$ & $18.6(11.5)$ \\
\hline RSS & $40.2(10.0)$ & $36.5(10.3)$ & $43.4(10.0)$ & $36.0(9.8)$ \\
\hline PSWQ & $59.2(8.5)$ & $55.0(9.8)$ & $60.6(11.8)$ & $51.6(11.3)$ \\
\hline KIMS observe & $39.2(8.2)$ & $39.5(8.7)$ & 40.1 (7.9) & $44.7(5.8)$ \\
\hline KIMS describe & $26.2(7.3)$ & $26.7(6.4)$ & $26.8(5.6)$ & $29.3(5.4)$ \\
\hline KIMS act aware & $26.3(6.9)$ & $26.5(6.7)$ & $25.9(6.7)$ & $29.5(4.7)$ \\
\hline KIMS accept & $28.9(7.3)$ & $31.7(7.3)$ & $26.6(8.4)$ & $30.7(7.7)$ \\
\hline PA & $3.9(.8)$ & $3.8(.9)$ & $3.5(.8)$ & $3.9(1.0)$ \\
\hline NA & $1.8(.5)$ & $1.7(.6)$ & $2.1(.7)$ & $1.8(.7)$ \\
\hline
\end{tabular}

Note. $\mathrm{MBCT}=$ Mindfulness-Based Cognitive Therapy; CONTROL = waitlist control condition; Pleasantness = Appraisal of activity pleasantness; HDRS=Hamilton Depression Rating Scale; IDS-SR = Inventory of Depressive Symptoms Self-Rating; RSS = Rumination on Sadness Scale; PSWQ $=$ Penn State Worry Questionnaire; KIMS = Kentucky Inventory of Mindfulness Skills; PA = Positive Affect; NA = Negative Affect. 


\section{Effects of treatment condition on residual depressive symptoms}

Table 2 shows pre- and postintervention means of primary and secondary outcome measures by number of episodes. As displayed in Table 3, the interaction between treatment condition (MBCT vs. CONTROL) and number of episodes (2- vs. $3+$ ) on reduction of residual depressive symptoms was not significant, indicating that the effect of MBCT does not depend on the number of depressive episodes. This was true for both the HDRS and the IDS-SR scores. Removing the non-significant interaction term revealed a main effect of treatment condition (HDRS: $d=-.56,95 \% \mathrm{Cl}[-.87,-.25], \mathrm{t}(126)=3.56, \mathrm{p}<.001$ ); IDS-SR: $d=-0.44,95 \% \mathrm{Cl}[-.71,-.18], \mathrm{t}(125)=3.27, \mathrm{p}<.001)$ but not of number of episodes ( $p$ for both HDRS and IDS-SR $>.20$ ). Thus, MBCT was associated with a significantly stronger reduction of residual depressive symptoms across the whole sample. Examination of effect sizes within the two subgroups (shown in Table 4) revealed that the effect of $M B C T$ relative to CONTROL was more pronounced in the 2-group compared to the $3+$ group. This indicates that the non-replication of higher efficacy in the $3+$ group cannot be attributed to low power. Figure 2 shows changes in residual depressive symptoms by treatment condition and number of episodes.

\section{Effects of treatment condition on secondary outcome measures}

In analyses of secondary outcome measures (positive and negative emotions, rumination, worry, and mindfulness skills), the effect of MBCT similarly did not depend on the number of prior episodes. The interaction between treatment condition and number of prior episodes was not significant for any of the variables, see Table 3. Again, there was a main effect of treatment condition across the whole sample: MBCT resulted in significant increases in positive emotions and mindfulness skills, and significant decreases in negative emotions, rumination, and worry (see also Geschwind, et al., 2011b). As shown in Table 4, effect sizes for the effect of treatment (MBCT vs. CONTROL) on rumination and worry were numerically larger in the 2 - group compared to the $3+$ group, even though these differences were not statistically significant. 
Table 3

Testing the interaction term "treatment condition $\mathrm{x}$ number of prior episodes".

\begin{tabular}{lcccccc}
\hline Measure & $d$ & \multicolumn{2}{c}{$[95 \% \mathrm{Cl}]$} & $t / z^{a}$ & $d f$ & $p$ \\
\hline HDRS & .45 & -.18 & 1.07 & 1.41 & $125_{\mathrm{b}}$ & .16 \\
IDS-SR & .33 & -.22 & .89 & 1.18 & 124 & .24 \\
RSS & .34 & -.19 & .86 & 1.27 & 124 & .21 \\
PSWQ & .23 & -.24 & .71 & .97 & 124 & .33 \\
KIMS observe & .09 & -.44 & .61 & .33 & 124 & .74 \\
KIMS describe & .25 & -.17 & .67 & 1.19 & 124 & .24 \\
KIMS act aware & -.18 & -.74 & .38 & .64 & 124 & .52 \\
KIMS accept & -.27 & -.73 & .18 & 1.19 & 124 & .24 \\
PA & .08 & -.04 & .20 & 1.25 & - & .21 \\
NA & -.07 & -.19 & .05 & 1.07 & - & .28 \\
\hline
\end{tabular}

Note. Positive values for $d$ indicate a stronger reduction in MBCT vs. CONTROL in the 2-group than the $3+$ group, while negative values indicate a stronger increase in the 2 - group. $M B C T=$ Mindfulness-Based Cognitive Therapy; CONTROL = waitlist control condition; Pleasantness = Appraisal of activity pleasantness; HDRS = Hamilton Depression Rating Scale; IDS-SR = Inventory of Depressive Symptoms Self-Rating; RSS = Rumination on Sadness Scale; PSWQ = Penn State Worry Questionnaire; KIMS = Kentucky Inventory of Mindfulness Skills; PA = Positive Affect; NA = Negative Affect.

${ }^{a}$ For the multilevel measures positive and negative affect (PA and NA), the test statistics are zscores, for the other measures, the test-statistics are t-scores. ${ }^{b}$ One participant completed only the HDRS but not the other measures at postmeasurement. 
Table 4

Effect size of treatment (MBCT vs. CONTROL), stratified by number of prior episodes.

\begin{tabular}{|c|c|c|c|c|c|c|c|c|c|c|}
\hline \multirow{3}{*}{$\begin{array}{r}\text { Measure } \\
\text { HDRS }\end{array}$} & \multicolumn{5}{|c|}{2 or less prior episodes } & \multicolumn{5}{|c|}{3 or more prior episodes } \\
\hline & \multirow{2}{*}{$\frac{d}{-.74}$} & \multicolumn{2}{|c|}{$[95 \%$ CI] } & \multirow{2}{*}{$\frac{t / z^{a}}{3.49 * * *}$} & \multirow{2}{*}{$\frac{d f}{68}$} & \multirow{2}{*}{$\frac{d}{-.29}$} & \multicolumn{2}{|c|}{$[95 \% C I]$} & \multirow{2}{*}{$\frac{t / z^{a}}{1.13}$} & \multirow{2}{*}{$\frac{d f}{56_{\mathrm{b}}}$} \\
\hline & & -1.13 & -.35 & & & & -.80 & .22 & & \\
\hline IDS & -.60 & -.96 & -.23 & $3.29 * *$ & 68 & -.26 & -.71 & .18 & 1.19 & 55 \\
\hline RSS & -.61 & -.95 & -.27 & $3.59 * * *$ & 68 & -.26 & -.68 & .16 & 1.24 & 55 \\
\hline PSWQ & -.62 & -.93 & -.32 & $4.04 * * *$ & 68 & -.38 & -.76 & -.01 & $2.05^{*}$ & 55 \\
\hline KIMS observe & .56 & .22 & .91 & $3.29 * *$ & 68 & .65 & .24 & 1.06 & $3.19 * *$ & 55 \\
\hline KIMS describe & .09 & -.20 & .38 & .63 & 68 & .35 & .05 & .66 & $2.31 *$ & 55 \\
\hline KIMS act aware & .75 & .38 & 1.12 & $4.02 * * *$ & 68 & .57 & .15 & .99 & $2.7 * *$ & 55 \\
\hline KIMS accept & .38 & .07 & .68 & $2.43 *$ & 68 & .09 & -.25 & .44 & .54 & 55 \\
\hline PA & .36 & .82 & .44 & $8.97 * * *$ & - & .44 & .35 & .53 & $9.12 * * *$ & - \\
\hline NA & -.25 & -.33 & -.17 & $5.89 * * *$ & - & -.31 & -.40 & -.23 & $6.99 * * *$ & - \\
\hline
\end{tabular}

Note. All effects are in favour of MBCT, relative to CONTROL. MBCT = Mindfulness-Based Cognitive Therapy; CONTROL = waitlist control condition; Pleasantness = Appraisal of activity pleasantness; HDRS=Hamilton Depression Rating Scale; IDS-SR = Inventory of Depressive Symptoms Self-Rating; RSS = Rumination on Sadness Scale; $\mathrm{PSWQ}=$ Penn State Worry Questionnaire; KIMS = Kentucky Inventory of Mindfulness Skills; PA = Positive Affect; NA = Negative Affect.

${ }^{a}$ For the multilevel measures positive and negative affect (PA and NA), the test statistics are $z$-scores, for the other measures, the test-statistics are t-scores. ${ }^{b}$ One participant completed only the HDRS but not the other measures at postmeasurement.

${ }^{*} \mathrm{p}<.05 .{ }^{* *} \mathrm{p}<.01 . * * * \mathrm{p}<.001$. 


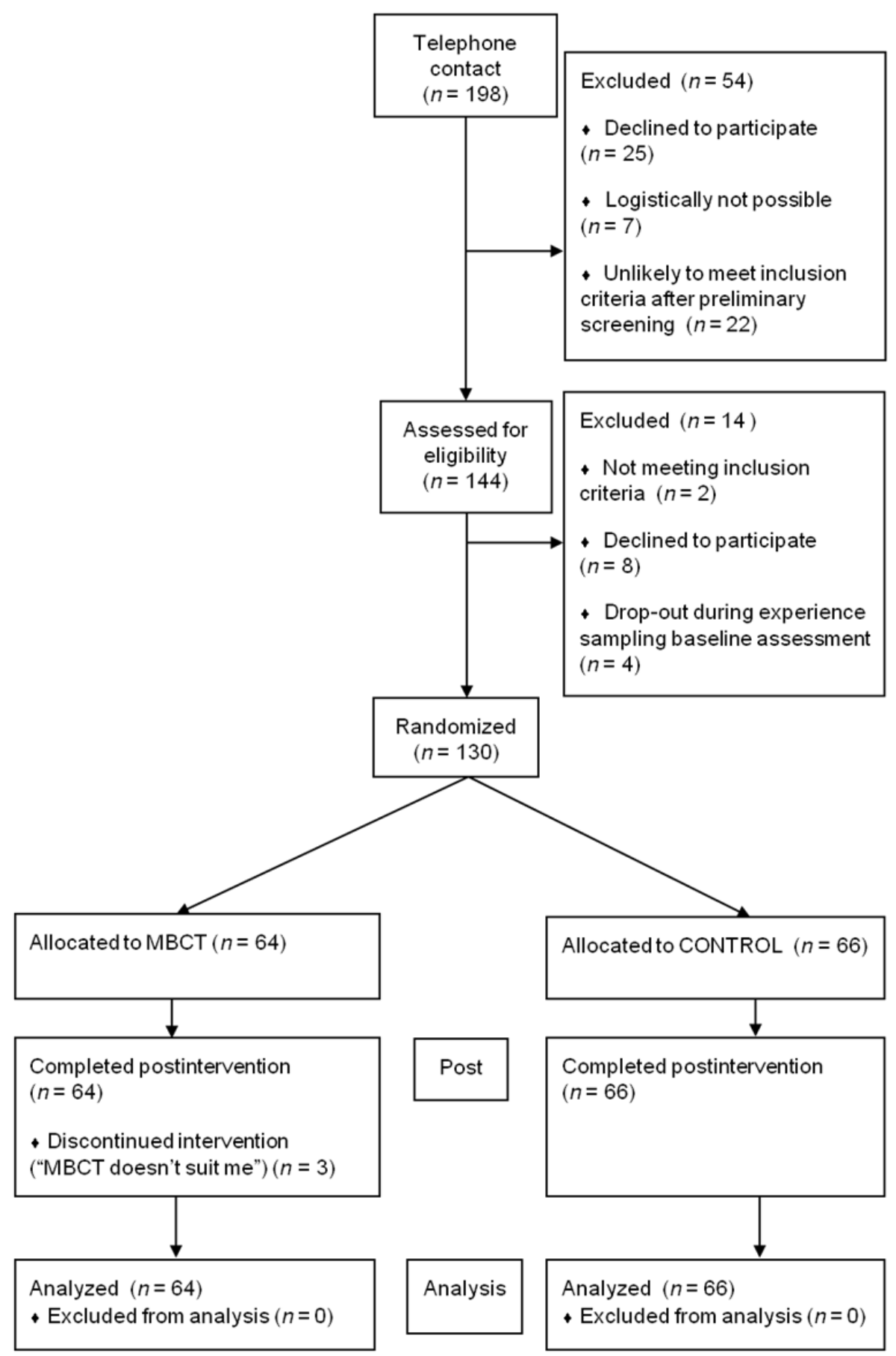

Figure 1. Participant flow diagram. $\mathrm{MBCT}=$ Mindfulness-Based Cognitive Therapy; $\mathrm{CONTROL}=$ waitlist control condition. 

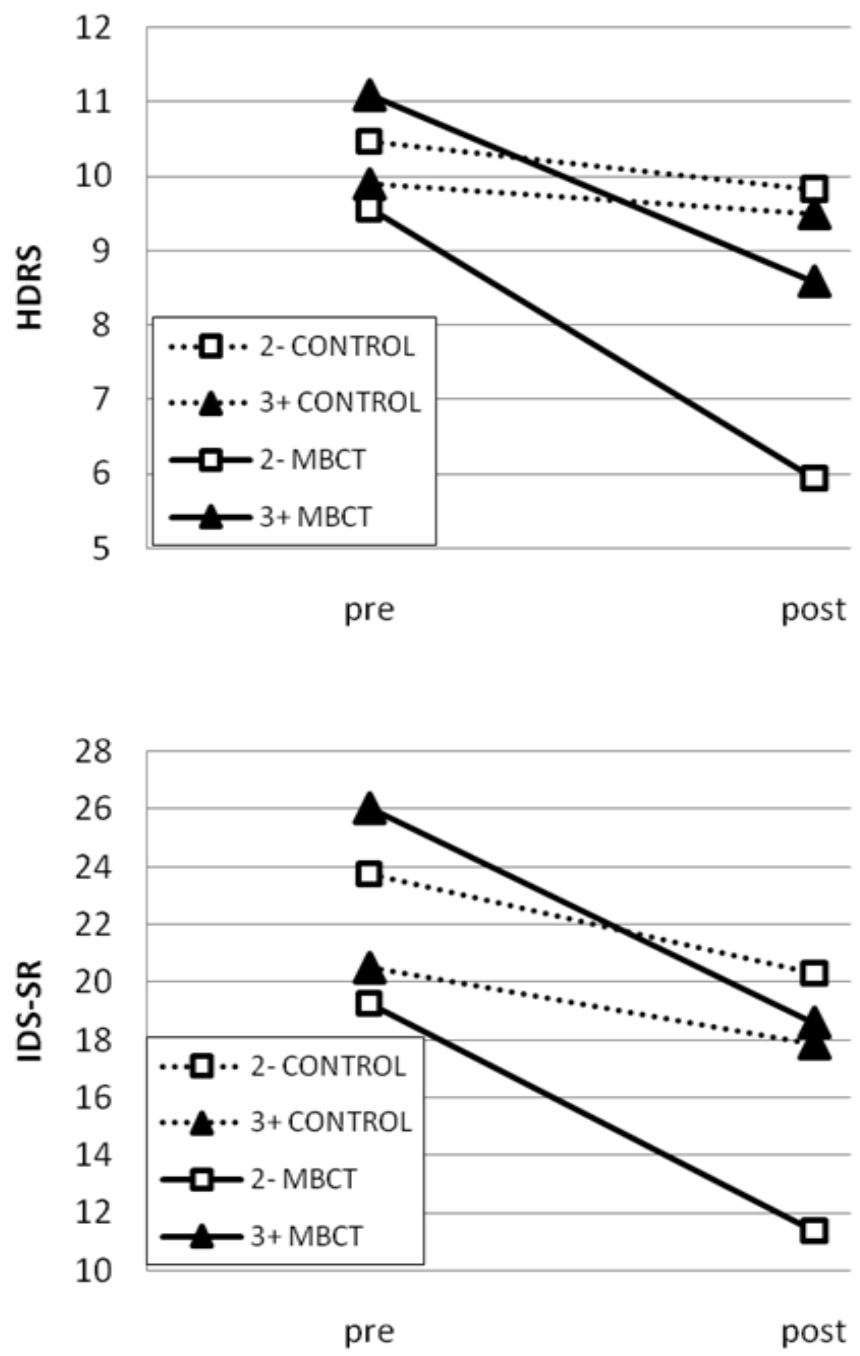

Figure 2. Change in residual depressive symptoms by treatment condition and number of prior episodes.

Note. 2 - = two or fewer prior episodes; $3+=$ three or more prior episodes; $\mathrm{MBCT}=$ MindfulnessBased Cognitive Therapy; CONTROL = waitlist control condition; HDRS=Hamilton Depression Rating Scale; IDS-SR = Inventory of Depressive Symptoms Self-Rating 


\section{DISCUSSION}

\section{Differential efficacy of MBCT by Subgroup?}

This randomized controlled trial found no evidence for differential efficacy on residual depressive symptoms of MBCT depending on the number of prior episodes (in line with Ramel, et al., 2004). Across the whole sample, MBCT led to significant reductions in residual depressive symptoms (approx. 30-35\% reduction), compared to CONTROL (approx. $10 \%$ reduction). Examination of effect sizes within subgroups revealed that non-replication of higher efficacy for $3+$ patients was not a result of low power, as the pattern of results was opposite to previous findings (Ma \& Teasdale, 2004; Teasdale, et al., 2000): Differences between MBCT and CONTROL were more pronounced in the 2-compared to the $3+$ group. Taken together, these results suggest that $\mathrm{MBCT}$ is indicated regardless of the 2- / 3+ distinction, at least as long as patients have residual symptoms. Findings therefore indicate an urgent need to reconsider the current practice of excluding patients with only one or two prior episodes of major depression from MBCT trials. Of course, one has to bear in mind that the outcome variables differed: residual symptoms in the current trial versus time to relapse in the original trials (Ma \& Teasdale, 2004; Teasdale, et al., 2000). However, evidence suggests that these outcomes are strongly related, residual symptoms being a powerful predictor of relapse (Judd, et al., 1999; Nierenberg, et al., 2010). Another major difference compared to the original trials is that the current trial explicitly included participants with residual symptoms (at least 7 on the HDRS, but not currently depressed according to the SCID-I interview), whereas the original trials excluded patients with residual symptoms (higher than 10 on the HDRS). Given recent findings that MBCT only worked in 3+ patients who at least occasionally experienced residual symptoms of depression (unstable remission; Segal, et al., 2010), it may well be that the efficacy of MBCT depends on the degree to which patients continue to experience residual symptoms, rather than on number of episodes.

\section{Different Mechanisms by Subgroup?}

Given that MBCT was originally assumed to primarily improve meta-cognition and other cognitive processes (reductions in rumination and worry) (Teasdale, et al., 2000), and given that cognitive processes appear to have a stronger influence during later episodes of relapse (Kendler, et al., 2000; Monroe \& Harkness, 2005), it may have been plausible to assume that MBCT works through different mechanisms in the different subgroups. For example, MBCT may lead to reduction in rumination and worry primarily in the $3+$ subgroup, and to improvements in 
emotion regulation primarily in the 2-subgroup. However, we found no indication for different mechanisms in the current trial. There was no evidence for an interaction between treatment group and number of depressive episodes regarding changes in rumination, worry, mindfulness skills and positive and negative emotions. On all these measures, MBCT was superior to CONTROL across the whole sample. Examining effects of MBCT by subgroup, effect sizes again were non-significantly larger for reductions in rumination and worry in the 2- group, compared to the $3+$ group. This finding is unexpected, given that subsequent episodes appear to be more related to internal cognitive processes than earlier episodes (Kendler, et al., 2000; Segal, et al., 1996). One therefore could have expected 3+ patients to show more improvement with regards to worry and rumination. On the other hand, there were no significant differences in worry and rumination between the two subgroups at baseline, so both subgroups had room for improvement. The presence of residual symptoms (which include rumination and worry) in the whole sample probably explains the lack of difference in rumination and worry between subgroups.

\section{Clinical Implications}

Clinical implications following from this trial are that $\mathrm{MBCT}$ treatment for residual symptoms should not be restricted to patients with 3 or more depressive episodes. As long as there are residual symptoms, MBCT appears to be superior to CONTROL on a number of outcomes. Although this trial did not directly examine risk for relapse/recurrence, reduction of residual symptoms can be expected to translate into reduced risk for relapse, considering how closely residual symptoms predict risk for relapse (Judd, et al., 1999; Nierenberg, et al., 2010). Beneficial changes not only included changes in meta-cognition but also emotional experience (i.e., more positive emotions, and less negative emotions), in line with previous suggestions and findings (Barnhofer, et al., 2007; Davidson, et al., 2003; Garland, et al., 2010). This is clinically important because (increases in) positive emotions have been found to predict recovery from depression and are associated with increased resilience in groups vulnerable to depression (Cohn, et al., 2009; Geschwind, et al., 2011b; Geschwind, et al., 2010).

\section{Strengths and limitations}

Strengths of the current study include a larger sample size for 2- patients $(n=71)$, compared to $\mathrm{Ma}$ and Teasdale (2004). Also, inclusion criteria were intentionally kept broad to enhance generalizability with respect to the general population of depressed patients. Finally, all randomized participants were assessed on the main outcome measure at baseline and post intervention (without attrition), and 
treatment compliance was high, with only three of $64 \mathrm{MBCT}$ participants attending less than four out of eight sessions.

Limitations include the fact that, despite the large sample size $(N=130)$, the power to detect an interaction between treatment condition and subgroup was low. However, non-replication of higher efficacy for $3+$ patients was not a matter of power, as effects actually were reversed, with higher efficacy for the 2subgroup. Second, because this trial examined reduction of residual symptoms immediately after the intervention, it still needs to be investigated if and how long after $\mathrm{MBCT}$ these improvements in clinical state remain stable. A recent study suggests that improvements in depressive symptoms following MBCT may be relatively long-lasting (Mathew, et al., 2010). Finally, the open-label, non-blinded nature of the current trial may have led to biased findings favoring $\mathrm{MBCT}$ over CONTROL. However, the agreement between the interview-based and self-report measure of residual depressive symptoms weakens this concern.

\section{Conclusions}

The present trial provided no evidence for a differential effect of $M B C T$ on residual depressive symptoms favoring those with a history of three or more episodes of major depression. MBCT was superior to CONTROL independent of the number of prior depressive episodes. Effects were not limited to improvements in depressive symptomatology and meta-cognition (i.e., reduced worry and rumination), but extended to improvements in emotional experience as well. Following MBCT but not CONTROL, participants experienced significantly higher levels of positive and lower levels of negative emotions in the flow of daily life. These improvements in emotional experience may well contribute to the beneficial effects of $M B C T$, as described in more detail in a previous report (Geschwind, et al., 2011b). In terms of reduction of residual depressive symptoms, effect sizes of MBCT compared to CONTROL even were about twice as large in participants with two or fewer prior episodes of depression, compared to three or more. Results therefore call into question the recent practice of excluding patients with only one or two prior episodes of depression from MBCT trials and treatment, and suggest that further examination of Teasdale and colleagues' initial findings of differential efficacy $(2004 ; 2000)$ is necessary. Finally, results indicate that it may be the degree to which patients continue to experience residual depressive symptoms after recovery from a depressive episode, rather than the number of prior episodes, which determines the efficacy of MBCT (in line with recent findings of Segal, et al., 2010). 


\section{Acknowledgements}

Marieke Wichers was supported by the Dutch Organisation for Scientific Research (NWO, VENI Grant Nr. 916.76.147). We thank our study participants for their time and effort. Furthermore, we thank Rufa Diederen for her help with data collection, and Truda Driesen, Philippe Delespaul, Frieda van Goethem, and the $4 \mathrm{D}$ database team for their help with data entry. 


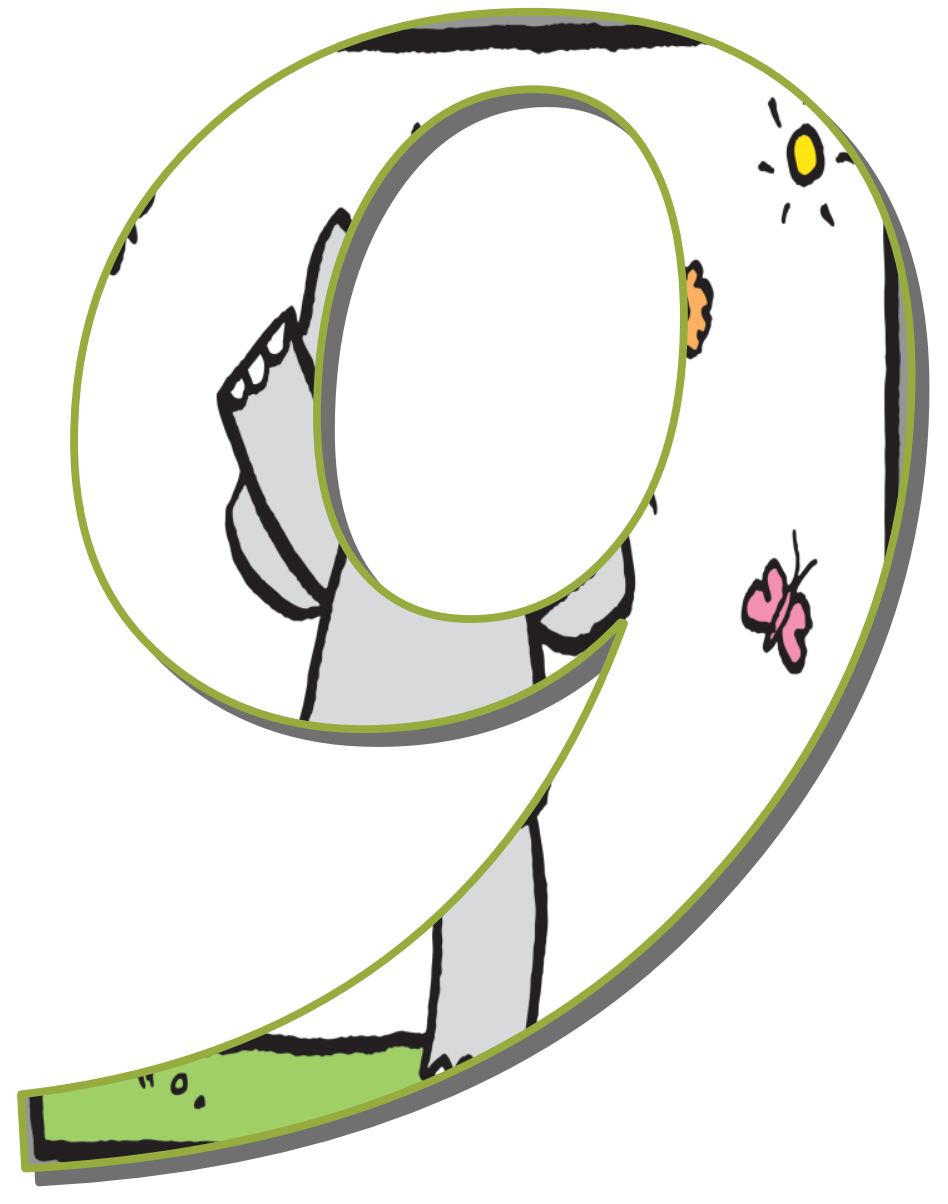

General Discussion 
The aim of the current thesis was to present research on vulnerability to and resilience against depression, with a focus on the role of affective processes as they occur in the flow of daily life. First, we discussed affective mechanisms involved in the development of depression. Then we moved on to investigate the role of daily-life emotions in the prediction of response during the early stages of treatment against depression. Based on these findings, we explored a new way to increase emotional resilience. The present thesis thus covers a range of topics, from etiology via response-prediction to intervention.

The overall discussion highlights selected findings and extrapolates conclusions to accommodate the discussion of societal, therapeutic, and research-related implications.

\section{THE ROLE OF AFFECTIVE PROCESSING IN THE ETIOLOGY OF DEPRESSION}

\section{Vulnerability}

Throughout this thesis, stress sensitivity (defined as high NA reactivity to stressful daily-life situations) has been established as a vulnerability phenotype for depression. Chapter 2 provided an overview of earlier publications discussing the role of stress sensitivity in depression. For example, study participants with a higher genetic risk of becoming depressed (measured by co-twin depression status) reacted to daily-life stressors with more NA than did participants with lower genetic risk, independent of their own level of depressive symptoms (Wichers, et al., 2007b). Furthermore, individuals with high baseline stress sensitivity were more likely to transgress to a depressive state, especially when environmental or genetic risk factors were present (Chapter 4). These studies suggest that high stress sensitivity represents vulnerability for depression, rather than purely being a consequence of being depressed.

\section{Resilience}

As discussed in Chapter 2, several findings implicate positive affect (PA) and reward experience (defined as PA reactivity to pleasant daily-life situations) in resilience against depressive complaints. For example, earlier experience sampling twin studies showed that momentary positive emotions provide a buffer against the emotional effects of stressful events in daily life (Wichers, et al., 2007b). The experience of positive emotions also attenuated the expression of genetic vulnerability on negative mood bias in the flow of daily life. The increased stress sensitivity associated with genetic risk for depression was less pronounced during the experience of positive emotions (Wichers, et al., 2008b; Wichers, et al., 
2007a). The experience of positive emotions thus seems to counter the expression of stress sensitivity, a vulnerability phenotype.

Furthermore, reward experience also seems to slow the transgression from vulnerability to a depressive state. Specifically, we found that reward experience appeared to be protective against the development of affective symptoms after exposure to environmental adversity (Chapter 6). Moreover, early changes in positive affect (PA) were a better predictor of recovery from depression than early changes in negative affect (NA). Our results thus link PA with resilience against depression, in line with Barbara Fredrickson's Undoing Hypothesis of positive emotions (Fredrickson, Mancuso, Branigan, \& Tugade, 2000). According to the Undoing Hypothesis, PA has the potential to undo the lingering aftereffects of negative emotional states. For example, Fredrickson and colleagues showed that people's cardiovascular reaction to stressful situations recovered much faster when they viewed PA-inducing rather than sadness-inducing or neutral film clips. This thesis extends the undoing effects of positive emotions to the domain of mental health.

Taken together, NA-related processes appear to be involved especially in vulnerability to depressive symptoms. PA-related processes, on the other hand, seem to be important in the prevention of and the recovery from depressive symptoms, as shown in Figure 1. This suggests that vulnerability and resilience are likely to be (at least partially) independent constructs rather than endpoints of a single dimension (similar to NA and PA, as discussed in the introduction).

\section{Continuous symptom dimensions rather than diagnostic categories}

In Chapter 4, higher stress sensitivity significantly predicted increased continuous depressive symptoms both by itself and in interaction with environmental or genetic risk for depression. The analyses on follow-up diagnoses of depression were much less sensitive, emphasizing the value of continuous measurement of symptoms. These findings therefore support the use of symptom dimensions, rather than diagnostic categories.

Another finding supporting a continuous view of symptom severity is that MBCT-related improvements were not restricted to patients with three or more prior episodes of major depression (Chapter 8). This means that improvements did not depend on a count of earlier episodes based on a categorical cut-off point, as Teasdale and colleagues have postulated earlier (Ma \& Teasdale, 2004; Teasdale, et al., 2000). Our finding is in line with results of Segal and colleagues (Segal, et al., 2010), who found that MBCT was only effective in patients who experienced residual depressive symptoms during remission. 


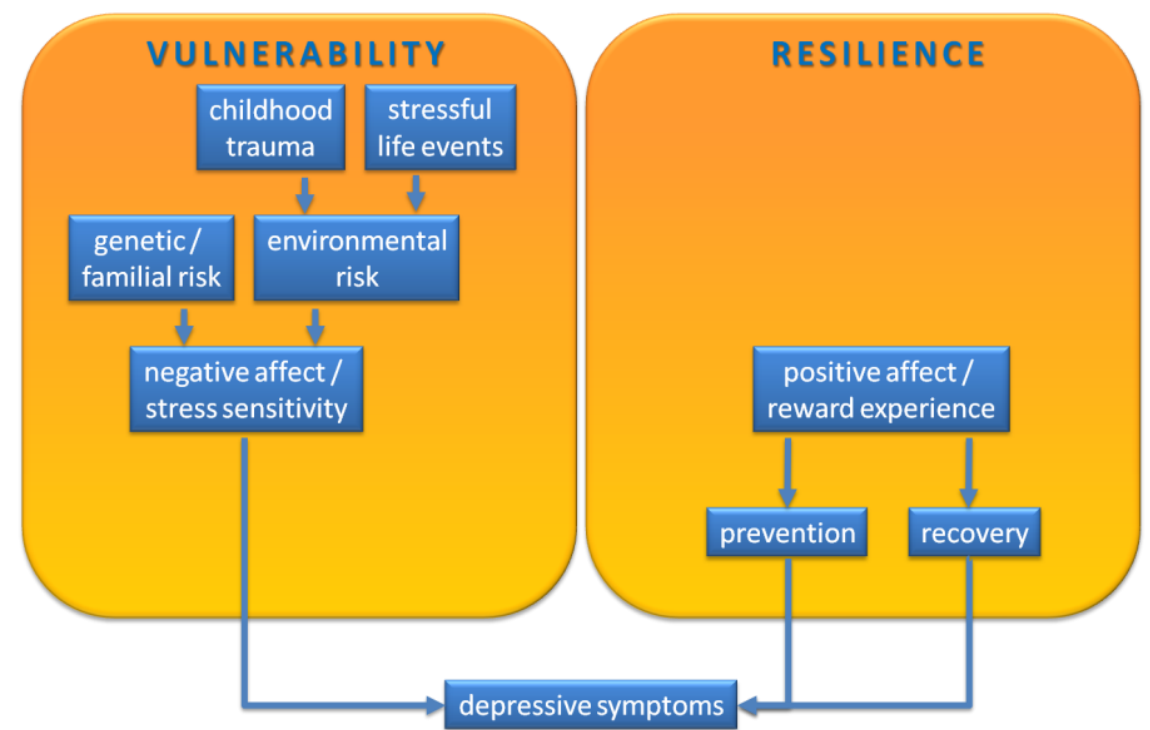

Figure 1. Vulnerability to and resilience against depression as (partially) independent constructs.

A continuous approach to depressive symptomatology may particularly benefit research examining scar theories. As discussed in Chapter 3, it is improbable that cognitive, emotional, behavioral or biological scars would only develop after an episode that fulfilled the criteria lined out in the Diagnostic Statistical Manual of Mental Disorders. More likely, if any scars remained, they would develop gradually and proportionally to the severity of depressive symptoms experienced. Researchers have proposed that such scars may explain why major stressful life events become progressively less associated with recurrences, compared to first-episode onsets of depression (Burcusa \& lacono, 2007; Lewinsohn, et al., 1981). A question unanswered so far is whether the decreased role of stressful life events in recurrent depression means that stressful life events lose the capacity to trigger depression, or whether they remain capable of triggering depression but are bypassed by other causes. Another possibility is that this decreased association in recurrence risk research is a purely statistical phenomenon related to the need to select patients based on the occurrence of an earlier episode (index event bias; Dahabreh \& Kent, 2011).

\section{Sensitization: Smaller stressors take over}

The current thesis supports the view that major stressful life events remain capable of triggering depression (Monroe \& Harkness, 2005) but have to compete 
with the increasing capacity of smaller stressors to similarly trigger depressive complaints (Chapter 4).

Arguments include the finding that participants with a history of major depression had higher baseline stress sensitivity, indicative of behavioral sensitization and scarring. Higher baseline stress sensitivity, in turn, predicted new episodes of depression. Finally, the role of post-baseline stressful life events was less pronounced at higher levels of stress sensitivity (in line with Kendler, et al., 2000). Further support for this hypothesis comes from an earlier study indicating that increased stress sensitivity likely can be attributed to stress exposures during development (Wichers, et al., 2009c).

\section{FROM ETIOLOGY TO INTERVENTION}

Findings outlined above favor a model viewing vulnerability and resilience as partially different constructs and implicate a larger role of positive rather than negative emotions in resilience-related processes. We therefore propose that interventions to increase resilience against depressive symptoms should focus on positive, rather than negative emotions (see Figure 2).

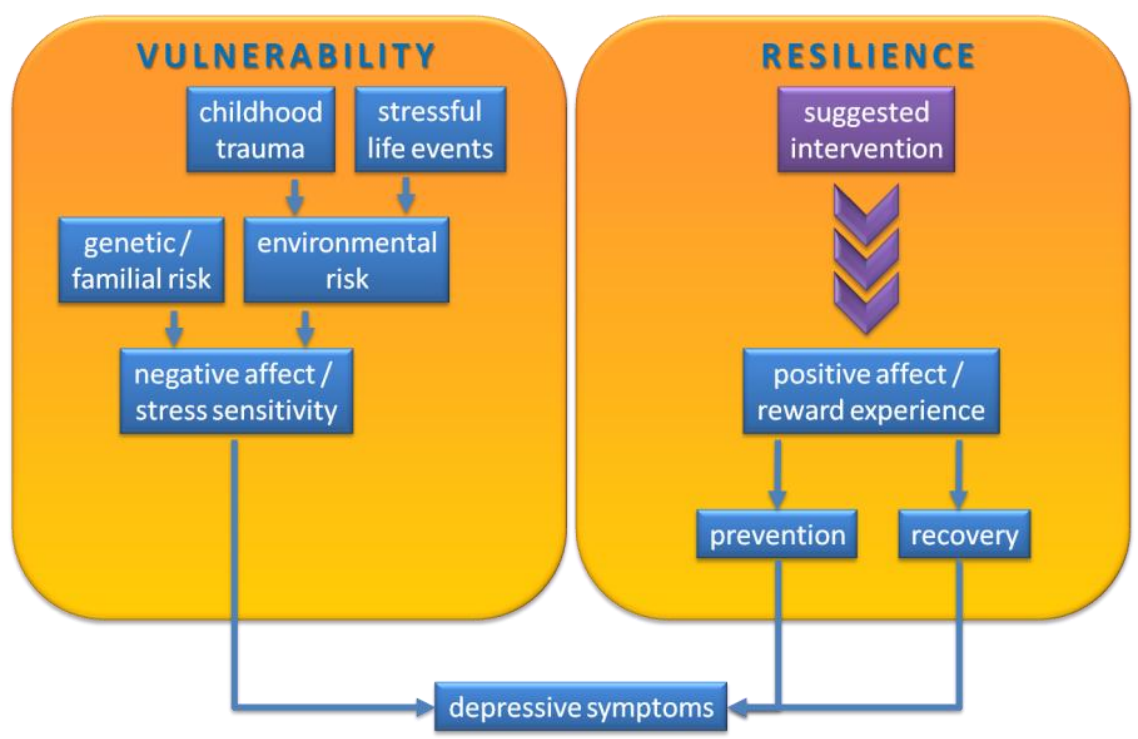

Figure 2. Suggested focus of interventions aimed at resilience. Interventions to increase resilience against depressive symptoms should focus on positive rather than negative affect, given that vulnerability and resilience are likely to be (partially) different and interacting constructs, rather than endpoints of one single continuum. 


\section{Can a behavioral intervention increase the capacity for positive affect?}

As discussed in this thesis, findings from experience sampling studies show that daily-life positive emotions have various beneficial effects with regards to mental health and resilience. People with a higher capacity for experiencing positive emotions seem to be more resilient against the development of depressive complaints compared to people with a lower capacity (Geschwind, et al., 2010). The ability to detect pleasant daily-life events or situations, and to use them as sources of positive affect, be it consciously or unconsciously, may thus help to counter the development of a depressive state. The important question then is whether this ability can be learned.

Research suggests a bidirectional link (an upward spiral) between broadened state of mind and positive affect (Fredrickson, 2004; Garland, et al., 2010). A broadened state of mind may consequently facilitate the experience of positive affect. Mindfulness-based Cognitive Therapy (MBCT) is a group-training program which blends meditational exercises with features of cognitivebehavioral therapy (Segal, et al., 2002b). The main goal of MBCT is to learn to focus on the here-and-now and to develop a mild, curious, and open attitude (Kabat-Zinn, 1990). In other words, participants train to develop increased moment-to-moment awareness in combination with an open mindset. These abilities may ease the detection of fleeting pleasant situations in daily life, as well as heighten the capacity to generate positive emotions. We therefore tested whether participation in MBCT was associated with an increased experience of positive affect and reward in daily life.

Our randomized controlled trial confirmed that only MBCT but not waitlistcontrol participants experienced greater appreciation for pleasant daily-life activities, higher overall levels of positive affect and increased PA-responsiveness to pleasant activities. Results therefore suggest that (i) people can learn to detect pleasant everyday situations and to use their rewarding properties to experience more positive emotions, and (ii) that MBCT may give rise to this process. Increases in PA-related variables were associated with decreases in residual depressive symptoms, underscoring the importance of positive emotion in resilience in depression.

\section{Positive emotion overkill?}

So far, we have established that positive emotions are associated with increased resilience against depression, and that $\mathrm{MBCT}$ may be a venue through which the capacity to experience positive emotions in daily life may be increased. A relevant question is whether interventions to stimulate positivity may backfire, for example by tipping the scales too much towards positive emotions, resulting in 
unbalanced affective processing. After all, a certain amount of short-lived negative emotion may be necessary to adapt to changing circumstances, motivate withdrawal behavior and signal that changes in behavioral strategy are needed (Fredrickson \& Losada, 2005).

Two phenomena are relevant to the discussion of this topic, namely negativity bias and positivity offset. Negativity bias refers to the finding that negative emotions or events are more noticeable than positive emotions or events (Baumeister, et al., 2001; Cacioppo, Gardner, \& Berntson, 1999). Positivity offset refers to the finding that, on average, people report to feel mildly positive, rather than neutral (Cacioppo, et al., 1999).

Using weighted counts of emotion ratings (to take negativity bias and positivity offset into account), Fredrickson and Losada have found that positivity:negativity ratio of approximately $3: 1$ seems to distinguish flourishing from languishing individuals (Fredrickson \& Losada, 2005). Individuals at risk for depression operate at lower-than-normal ratios, and remission from depression is associated with more optimal ratios (Schwartz, et al., 2002). However, research shows that problems can also occur with too much positivity, manifesting as states of grandiosity, denial, or mania (Schwartz, et al., 2002). Mathematical models show that behavior patterns become rigid and inflexible at ratios above 11:1 (Fredrickson \& Losada, 2005). These findings thus indicate that too much positive affect can also be problematic. However, it is unlikely that a behavioral intervention would result in such extreme increases in positive affect. Moreover, individuals at risk for depression have been shown to operate at lower-thannormal ratios (Schwartz, et al., 2002), so an intervention aimed at increasing the experience of positive emotions would probably only restore the balance to a more normal ratio. On the other hand, research on brain patterns (Ekman, Davidson, \& Friesen, 1990) and heart functioning (Rosenberg et al., 2001) suggests that pretending to be feel good may also backfire, implying that the genuineness of positive emotions is important.

These findings would support an intervention which is not too explicit and does not create a demand bias for positive emotions. In this respect, MBCT is a balanced intervention, because participants are not actively instructed to feel more positive. In contrast, they practice to adopt a state of mind (open and curious, with attention on the present moment) which is compatible with the detection of pleasant situations and the generation of positive emotions. Other subtle intervention options may include direct (neuro)feedback on physiological states which facilitate the generation of positive emotions. Feedback on vagal tone may be a promising candidate, given that researchers recently found a 
bidirectional link between increased vagal tone and positive emotions and social connectedness (Kok \& Fredrickson, 2010). Non-invasive, transcutaneous stimulation of the vagus nerve may be a further option. A recent study shows that participants of mindfulness but not relaxation training displayed increases in vagal tone (Delgado, et al., 2010), thus linking MBCT not only to psychological but also to physiological states associated with positive emotions.

\section{Personalized Medicine or Broad-scale Interventions?}

This thesis also touched on the issue of personalized medicine. Personalized medicine refers to the idea that interventions can be tailored to meet personspecific needs. Is there a role for personalized medicine with respect to interventions aimed at increasing resilience?

Findings that might speak in favor of the notion of personalized medicine based on genetics are the fact that specific genes (BDNF, 5-HTTLPR) as well as familial risk have been found to moderate the transition from stress sensitivity to a depressive state (Chapter 4). Also reward experience seems to be moderated by the COMT Val ${ }^{158}$ Met polymorphism (Chapter 2), a finding which has been replicated in the MindMaastricht data (unpublished data). On the other hand, high stress sensitivity predicted transition to a depressive state independently of genetic background, and so did stressful life events (especially when stress sensitivity was low; Chapter 4). Furthermore, estimates on the heritability of dailylife reward experience indicate that up to $85 \%$ of variation in reward experience is due to environmental influences (Lothmann, personal communication). So although genes may exacerbate the effects of other risk factors, the known contribution of specific genes is small, and vulnerable groups can be easily be identified at several other levels (e.g., high stress sensitivity, recent major stressful life events, or familial risk for depression). Moreover, genetic testing is expensive. These facts speak against a role for personalized medicine based on genetic variants.

Another type of personalized medicine may lie in the analysis of individual strength-and-weakness profiles. With respect to different domains (e.g., emotion, cognition, coping, social support) of resilience interventions, such a profile may determine which kind of intervention is most needed. With respect to affective processing, experience sampling may be useful in assessing who benefits most from interventions aimed at increasing reward experience. For example, people scoring low on positive affectivity may benefit most from an intervention aimed at reward experience, whereas another focus (e.g., on stress sensitivity) may be better for people already scoring high on positive affectivity. This topic, however, deserves further examination. 
The most evident conclusion following from the current thesis would be to make early broad-scale resilience interventions available for vulnerable groups. Indeed, it may be better to err on the positive side and to make broad-scale, lowthreshold interventions aimed at increasing resilience available to everyone. For example, resilience exercises may be integrated into everyday life from an early age on, starting in kindergartens and schools (Seligman, Ernst, Gillham, Reivich, \& Linkins, 2009). Work settings could be organized in a way that facilitates the experience of positive emotions (Burton, Pakenham, \& Brown, 2010; Waite \& Richardson, 2004). Even though not everyone may benefit (e.g., reward experience was associated with benefits only in individuals exposed to early or late environmental adversity; Chapter 6), such interventions are unlikely to do harm and likely to result in more resilient individuals, less depressive complaints, and better social contacts (Fredrickson, 2003).

\section{Directions for Future Research}

Several recommendations for future research follow from the studies presented here. First of all, in order to maximize knowledge gains and facilitate cross fertilization between different domains, the degree of correspondence between daily-life phenotypes (measured using momentary assessment techniques) and laboratory tasks will have to be investigated in more detail. For example, there is quite some research on laboratory measures of reward experience and involved brain regions and neurotransmitters. On the other hand, not much is known about how well laboratory reward measures correspond to daily-life reward experience, so the degree to which knowledge is transferable is currently unpredictable.

With regard to diagnostics and response prediction, it would be interesting to examine the role of individual profiles more closely. For example, to what extent do baseline characteristics predict the mechanism of improvement (e.g., increased reward experience, decreased stress sensitivity, improved cognition)? Strong associations between baseline characteristics and response mechanisms may favor more personalized interventions, while weak associations may point to the importance of a single underlying resilience mechanism.

With respect to treatment and intervention, a series of repeated single-case experiments investigating the effective ingredient in MBCT would be useful. MBCT could be split up into several component packages including behavioral activation, attention exercises, stress-reduction, breathing, and attitudinal education. These packages could then be scrambled, and one could track the effects of each package. Possibly, each package could also be compared to a bogus package in order to distinguish specific from nonspecific effects. 
Furthermore, the extent to which increased reward experience represents a mechanism of MBCT needs to be investigated in more detail. For example, an association between the extent of increase in reward experience and a more favorable future course of illness would support the hypothesis of reward experience as a mechanism of change of MBCT. A comparison with an active control intervention could establish the degree to which changes in positive emotion and reward experience are specific to increased mindfulness. Such a comparison may also reveal whether there are yet better methods of increasing the ability to experience positive emotions in daily life.

With regard to physiological facilitation of positive affect in daily life, it would be interesting to investigate to which extent direct manipulation of vagal tone leads to changes in positive affect and reward experience. For example, effects of real direct subcutaneous stimulation of the vagus nerve through a portable device (e.g., see http://www.cerbomed.com/product.html) could be compared to a bogus condition.

\section{CONCLUSIONS}

With regard to the etiology of depressive symptoms, the most important conclusions from this thesis are that (1) high levels of negative emotion and stress sensitivity were involved in vulnerability to depressive symptoms. Furthermore, (2) high levels of positive emotions and reward experience were involved in resilience against depressive symptoms. Concerning the prediction of response to treatment, (3) early increases in positive rather than negative emotion predicted recovery from depression. Moreover, (4) adding measures of positive emotions to conventional measures of symptom improvement may improve clinical decisionmaking. Regarding resilience-related interventions, (5) participation in MBCT facilitated the experience of positive emotions in daily life, suggesting that MBCT is a promising intervention to increase emotional resilience against depressive symptoms. (6) Effects of MBCT were independent of the number of prior depressive episodes, suggesting that $\mathrm{MBCT}$ should be available to everybody in need of increased resilience. 


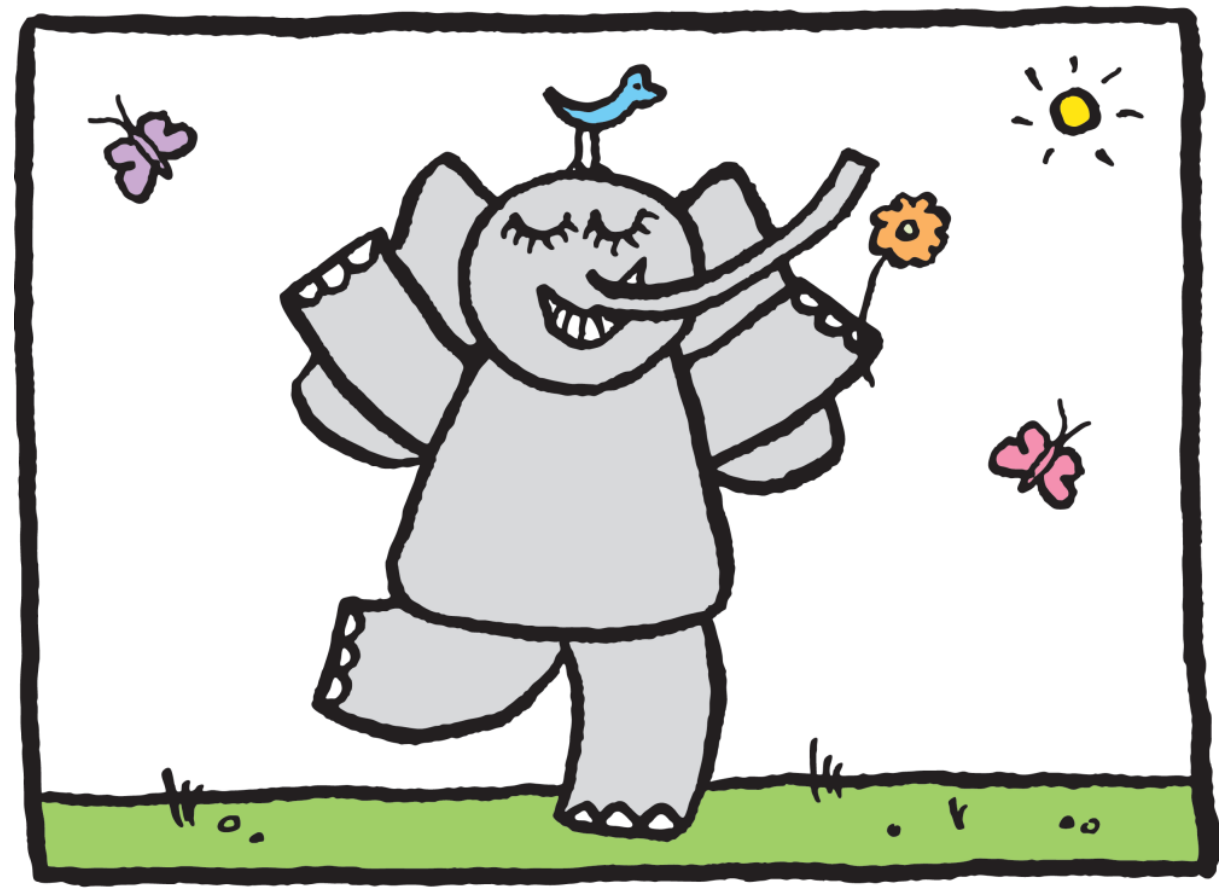

\section{Summany}


From vulnerability to resilience against depression. The value of daily-life positive emotions. This thesis presents experience sampling research on the role of affective processing in vulnerability to and resilience against depressive symptoms. We first examined the role of stress sensitivity in vulnerability to depressive symptoms. Second, we moved on to affective processing involved in resilience against depressive symptoms. Then we investigated the role of early change in positive and negative emotions in predicting recovery from depression. Finally, based on these earlier studies, we presented a novel way to increase emotional resilience against depressive symptoms. In short, we progressed from etiology to intervention, focussing on the value of positive emotions in daily life.

Chapter 1 presents some background information on depressive disorders and affective processes in daily life and introduces the experience sampling method (ESM) used in most of the studies. Furthermore, Chapter 1 provides an outline of the current thesis.

Chapter $\mathbf{2}$ is an extension to the general introduction. Several examples illustrate the complex nature of the diagnostic concept "depression", with interacting genetic and environmental influences. The advantages of examining more proximal intermediate phenotypes, rather than diagnostic categories, are discussed, as well as the potential of ESM to contribute to valid measurements of such intermediate phenotypes. The main idea put forward in Chapter 2 is that stress sensitivity (i.e., negative affect reactivity to stressful daily-life situations) may be an intermediary phenotype associated with vulnerability to depressive symptoms. Positive affect and reward experience (positive affect reactivity to pleasant daily-life situations), on the other hand, are likely to be associated with resilience against depressive symptoms. The chapter closes by highlighting the need for further research on the role of positive emotions in resilience against depressive symptoms.

Chapter $\mathbf{3}$ is a conceptual article which outlines a new approach to the examination of scar theories. Scar theories assume that some trace remains after recovering from a depressive episode, predisposing recovered individuals to relapse or recurrence. However, research on biological, psychological, or cognitive changes has remained largely inconclusive. Chapter 3 proposes a new approach to the concept of 'scar' that is based on a dimensional view of depression and uses methods that take into account the dynamic interplay between a person and his/her environmental context. The new approach furthermore differentiates 
between scars following depression and scars following the factor that actually caused the depression (e.g., stressors). Finally, the new approach proposes a dynamic rather than static view of the concept of 'scar', namely that scars can wax and wane.

Chapter 4 follows up on the idea of stress sensitivity as an intermediary phenotype of depression, using data from a prospective longitudinal study on approximately 500 twins. We examined associations between baseline daily-life stress sensitivity and later depressive state, taking genetic and non-genetic factors into account. Higher stress sensitivity at baseline predicted more depressive symptoms and transition to major depressive disorder at follow-up. Genetic vulnerability increased the probability of transition from stress sensitivity to a depressive state. When stress sensitivity was high, the harmful influence of stressful life events on depressive symptoms was less pronounced. Onset of depression thus is partially attributable to pre-onset measures of stress sensitivity, supporting the idea of stress sensitivity as an intermediary phenotype of depression.

In Chapter 5, we examined affective mechanisms involved in recovery from depression in a sample of 49 depressed patients. Specifically, we tested the association between changes in positive and negative emotions during the first week of pharmacological treatment and severity of depressive symptoms at week six. We found that early change in positive rather than negative emotions best predicted response to treatment, supporting the notion that antidepressants activate resilience-like mechanisms. Effects of early change in negative emotions were only half as large and disappeared when evaluated simultaneously with early change in positive emotions. Monitoring of positive emotions in early stages of treatment furthermore contributed over and above outcome prediction using the conventionally employed Hamilton Depression Rating Scale (HDRS; Hamilton, 1960). Results thus support the notion that positive emotions in particular are involved in resilience and recovery. Moreover, monitoring of positive emotions in early stages of treatment may improve clinical decision making.

Chapter 6 presents results of a prospective twin study on daily-life reward experience and resilience against affective symptoms. We found that high reward experience at baseline was associated with reduced future affective symptoms in vulnerable participants (i.e., those with previous exposure to childhood adversity or stressful life events). High daily-life reward experience thus increases resilience 
after environmental adversity. Results raise the question whether people can learn to get more out of pleasant daily-life experiences and point to a novel area of therapeutic intervention (aimed at modification of reward experience).

In Chapter 7, we investigated whether Mindfulness-Based Cognitive Therapy (MBCT) may be an intervention which increases momentary positive emotions and the ability to make use of natural rewards in daily life. MBCT is a group skillstraining program which combines meditation exercises with features of Cognitive Therapy. We compared MBCT to a waitlist control group in a randomized controlled trial on 130 participants with residual depressive symptoms. MBCT compared to a waitlist control condition was associated with significant increases in appraisals of positive emotion and activity pleasantness, as well as enhanced ability to boost momentary positive emotions by engaging in pleasant activities. Furthermore, increases in positive emotion variables were associated with decreases in residual depressive symptoms. From a clinical point of view, findings are relevant because they indicate that a non-pharmacological intervention may potentially improve the reward system. Also, improvements in affective resilience phenotypes may contribute to the (by now well-documented) protective effects of MBCT against depressive relapse.

Chapter 8 further examined the effects of MBCT. Until now, patients with only one or two prior episodes of major depression were usually excluded from trials investigating the effects of $\mathrm{MBCT}$ on depression (based on findings from two earlier studies). However, several arguments support the thesis that this exclusion may have been premature: Effects of $\mathrm{MBCT}$ are diverse and not restricted to patients with prior depressive episodes. Furthermore, we showed that MBCT facilitated an affective processing style associated with increased resilience. MBCT should therefore be beneficial for anyone at risk for depression (independent of the number of prior depressive episodes). Indeed, we found no evidence for increased efficacy of $\mathrm{MBCT}$ in participants with three or more compared to two or less prior episodes in our sample. Clinical implications following from this trial are that participation in $\mathrm{MBCT}$ trials and treatment should not be restricted to patients with at least three depressive episodes. Instead, treatment should be available to anyone in need of increased resilience against depressive complaints.

Chapter 9 provides a general discussion of the studies included in this thesis, along with clinical and societal implications and suggestions for future research. The main conclusion is that vulnerability and resilience represent (at least 
partially) independent constructs, with positive emotions being especially involved in processes related to resilience. Therefore, interventions to enhance resilience against depressive complaints are likely to be more successful when they focus on positive, rather than negative emotions. 



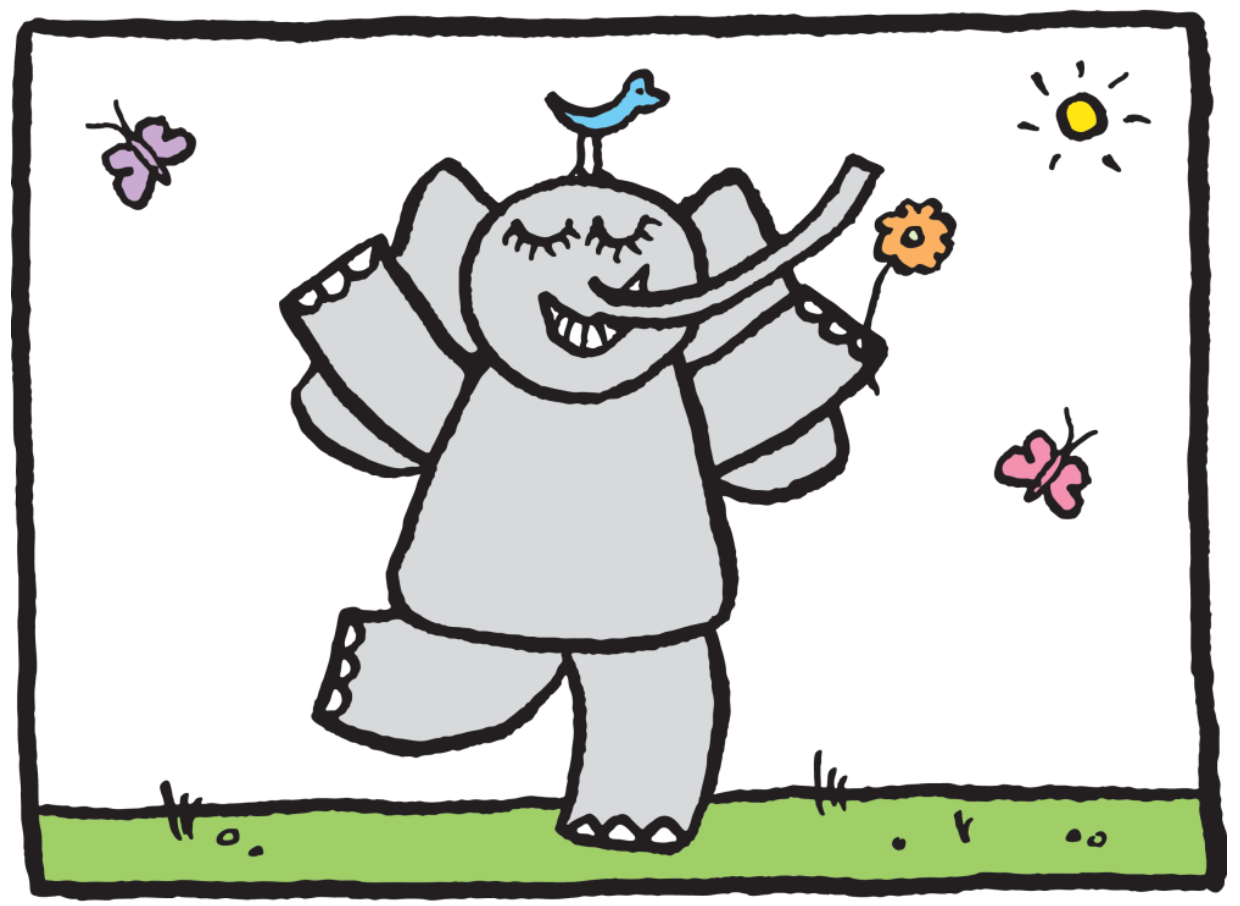

Samenvatting 
From vulnerability to resilience against depression. The value of daily-life positive emotions. Dit proefschrift presenteert Experience Sampling onderzoek naar de rol van affectieve processen in kwetsbaarheid voor en veerkracht tegen depressieve symptomen. We hebben eerst de rol van stressgevoeligheid in het dagelijkse leven als voorspeller van kwetsbaarheid voor depressieve symptomen onderzocht. Hierna onderzochten wij in hoeverre affectieve processen betrokken zijn bij veerkracht tegen depressieve symptomen. Vervolgens hebben we onderzoek gedaan naar de rol van vroege veranderingen in positieve en negatieve emoties in het voorspellen van herstel van depressie. Tot slot presenteerden wij, op basis van deze eerdere studies, een nieuwe manier om emotionele veerkracht tegen depressieve klachten te verhogen. Kortom, we begonnen met onderzoek naar de etiologie van depressieve symptomen en eindigden met een interventie gericht op het verhogen van positieve emoties in het dagelijks leven.

Hoofdstuk 1 geeft achtergrondinformatie over depressieve stoornissen en affectieve processen in het dagelijkse leven en introduceert de Experience Sampling Methode (ESM) die in de meeste studies in dit proefschrift gebruikt is. Bovendien geeft Hoofdstuk 1 een overzicht van het huidige proefschrift.

Hoofdstuk 2 is een uitbreiding op de algemene inleiding. Een aantal voorbeelden illustreert de complexe aard van de diagnosecategorie "depressie", waarin genetische invloeden en ook omgevingsinvloeden een rol spelen. De voordelen van het onderzoeken van meer proximale tussenliggende fenotypes, in plaats van diagnostische categorieën, worden besproken, evenals het potentieel van ESM om bij te dragen aan een valide meting van dergelijke intermediaire fenotypes. Het belangrijkste idee dat in hoofdstuk twee naar voren wordt gebracht is dat stressgevoeligheid (dat wil zeggen, een sterke stijging in negatieve emoties als gevolg van stressvolle situaties uit het dagelijkse leven) een intermediair fenotype van kwetsbaarheid voor depressieve symptomen zou kunnen zijn. Positief affect en het ervaren van beloning (een sterke stijging van positieve emoties als gevolg van aangename situaties uit het dagelijkse leven), aan de andere kant, kan in verband worden gebracht met veerkracht tegen depressieve symptomen. Het hoofdstuk sluit af door te wijzen op de noodzaak van verder onderzoek naar de rol van positieve emoties in weerbaarheid tegen depressieve symptomen.

Hoofdstuk 3 is een conceptueel artikel dat een nieuwe benadering schetst van onderzoek naar "scar" (litteken) theorieën. Scar theorieën gaan ervan uit dat een 
litteken overblijft na herstel van een depressieve episode. Dit litteken zou herstelde individuen predisponeren om opnieuw een depressie te krijgen. Echter bleef onderzoek naar de biologische, psychologische of cognitieve veranderingen grotendeels zonder resultaten. Hoofdstuk 3 stelt een nieuwe benadering van het littekenconcept voor. De voorgestelde benadering is gebaseerd op een continuümvisie van depressieve symptomen (meer of minder klachten in plaats van wel of niet depressief) en maakt gebruik van methodes die rekening houden met de dynamische wisselwerking tussen een persoon en zijn of haar omgeving. De nieuwe aanpak maakt bovendien onderscheid tussen littekens als gevolg van depressie en littekens als gevolg van factoren die een depressie kunnen uitlokken (bijv. stressoren). Ook heeft de voorgestelde aanpak een dynamische in plaats van een statische opvatting van het begrip "litteken", namelijk dat littekens kunnen groeien en krimpen.

Hoofdstuk 4 gaat verder in op het idee van stressgevoeligheid als een intermediair fenotype van depressie, met behulp van gegevens uit een prospectieve longitudinale studie waar ongeveer 500 tweelingen aan deelnamen. Wij onderzochten associaties tussen stressgevoeligheid in het dagelijkse leven bij begin van de studie en latere depressieve toestand, waarbij we rekening hielden met genetische en niet-genetische factoren. Een hogere stressgevoeligheid voorspelde meer depressieve symptomen en een hoger risico op een klinisch relevante depressieve stoornis bij de vervolgmetingen. Genetische kwetsbaarheid hing samen met een verhoogde kans op transitie van stressgevoeligheid naar een depressieve toestand. Wanneer de stressgevoeligheid hoog was, was de schadelijke invloed van stressvolle gebeurtenissen op het ontstaan van depressieve symptomen minder sterk. Het ontstaan van een depressie is dus deels toe te schrijven aan eerdere (pre-depressieve) metingen van stressgevoeligheid. Deze bevindingen steunen het idee van stressgevoeligheid als een intermediair fenotype van depressie.

In hoofdstuk 5 onderzochten we affectieve mechanismen die betrokken zijn in het herstel van een depressie. In een steekproef van 49 depressieve patiënten testten we de associatie tussen veranderingen in positieve en negatieve emoties tijdens de eerste week van een farmacologische behandeling en ernst van depressieve symptomen in de zesde week. De resultaten lieten zien dat een vroege verandering van positieve in plaats van negatieve emoties een goede respons op de behandeling het beste voorspeld. De resultaten zijn dus compatibel met het idee dat antidepressiva veerkrachtachtige mechanismen activeren. Effecten van 
een vroege verandering in negatieve emoties waren slechts half zo groot en verdwenen wanneer ze gelijktijdig met een vroege verandering in de positieve emoties geëvalueerd werden. Het monitoren van positieve emoties in zo'n vroeg stadium van de behandeling droeg bovendien bij aan een verbeterde voorspelling van behandelrespons, vergeleken met alleen de Hamilton Depression Rating Scale (HDRS, Hamilton, 1960) die gewoonlijk wordt toegepast. De resultaten steunen dus het idee dat vooral positieve emoties betrokken zijn bij veerkracht tegen en herstel van depressie. Bovendien kan het monitoren van positieve emoties in vroege stadia van de behandeling mogelijk leiden tot een verbeterde besluitvorming met betrekking tot de behandeling van patiënten met depressieve klachten.

Hoofdstuk 6 presenteert de resultaten van een prospectieve tweelingstudie waarin we gekeken hebben naar de relatie tussen het ervaren van beloning in het dagelijkse leven en weerbaarheid tegen affectieve symptomen. We vonden dat een hoog vermogen om beloning te ervaren aan het begin van de studie geassocieerd was met verminderde affectieve symptomen in de toekomst. Wel was dit alleen het geval voor kwetsbare deelnemers (mensen die tegenslagen in de kindertijd of recente stressvolle levensgebeurtenissen hadden meegemaakt). Een hoog vermogen om beloning te ervaren is dus een teken van veerkracht. De resultaten roepen de vraag op of mensen zouden kunnen leren om meer voldoening te halen uit alledaagse plezierige ervaringen. Ook wijzen de resultaten naar een nieuwe invalshoek wat betreft therapeutische interventies (namelijk gericht op het verhogen van het ervaren van beloning).

In hoofdstuk 7 hebben we onderzocht of Mindfulness-Based Cognitive Therapy (MBCT) gebruikt zou kunnen worden om positieve emoties en het vermogen om voldoening te halen uit alledaagse plezierige ervaringen te vergroten. MBCT is een groepstrainingsprogramma dat meditatieoefeningen combineert met technieken uit de Cognitieve Therapie. We vergeleken MBCT met een wachtlijstcontrolegroep in een gerandomiseerd gecontroleerd onderzoek waar in totaal 130 deelnemers met restklachten van depressie aan deelnamen. In vergelijking met de wachtlijstcontrolegroep was MBCT geassocieerd met een significante stijging van positieve emoties en plezierige activiteiten, evenals een verbeterd vermogen om positieve emoties te genereren naar aanleiding van plezierige activiteiten. Verder waren stijgingen in variabelen gerelateerd aan positieve emotie geassocieerd met dalingen in residuële depressieve symptomen. Vanuit een klinisch standpunt zijn de bevindingen relevant omdat ze laten zien dat een niet-farmacologische 
interventie het vermogen om beloning te ervaren potentieel kan verbeteren. Ook zouden de verbeteringen in de affectieve fenotypes geassocieerd met veerkracht mogelijk een deel van de (inmiddels goed gedocumenteerde) beschermende effecten van MBCT tegen terugkerende depressie kunnen verklaren.

In hoofdstuk 8 hebben we de effecten van MBCT verder onderzocht. Tot nu toe werden patiënten met slechts een of twee eerdere episodes van depressie meestal uitgesloten van onderzoek naar de effecten van MBCT op depressie (gebaseerd op de bevindingen van twee eerdere studies). Verschillende argumenten ondersteunen echter de stelling dat deze uitsluiting onterecht is: effecten van MBCT zijn divers en niet beperkt tot patiënten met eerdere depressieve episodes. Verder hebben we laten zien dat MBCT een affectieve verwerkingsstijl vergemakkelijkt die geassocieerd is met een verhoogde veerkracht. MBCT zou dus gunstig moeten zijn voor iedereen met een verhoogd risico voor depressie (onafhankelijk van het aantal eerdere depressieve episodes). We vonden in onze steekproef inderdaad geen bewijs voor een verhoogde effectiviteit van MBCT bij deelnemers met drie of meer in vergelijking met twee of minder episodes. Klinische implicaties van deze studie zijn dat deelname aan MBCT onderzoek en behandeling niet beperkt zou moeten worden tot patiënten met minstens drie depressieve episodes. In plaats daarvan zou de behandeling beschikbaar moeten zijn voor iedereen die behoefte heeft aan grotere weerbaarheid tegen depressieve klachten.

In hoofdstuk 9 vindt een algemene bespreking van de studies in dit proefschrift plaats, worden klinische en maatschappelijke implicaties bediscussieerd en suggesties voor toekomstig onderzoek gedaan. De belangrijkste conclusie is dat kwetsbaarheid en veerkracht (tenminste gedeeltelijk) onafhankelijke constructen representeren. Positieve emoties lijken vooral betrokken te zijn bij processen die verband houden met veerkracht. Interventies om weerbaarheid tegen depressieve klachten te verbeteren zullen dan ook waarschijnlijk meer succes hebben als ze zich richten op positieve, in plaats van negatieve emoties. 



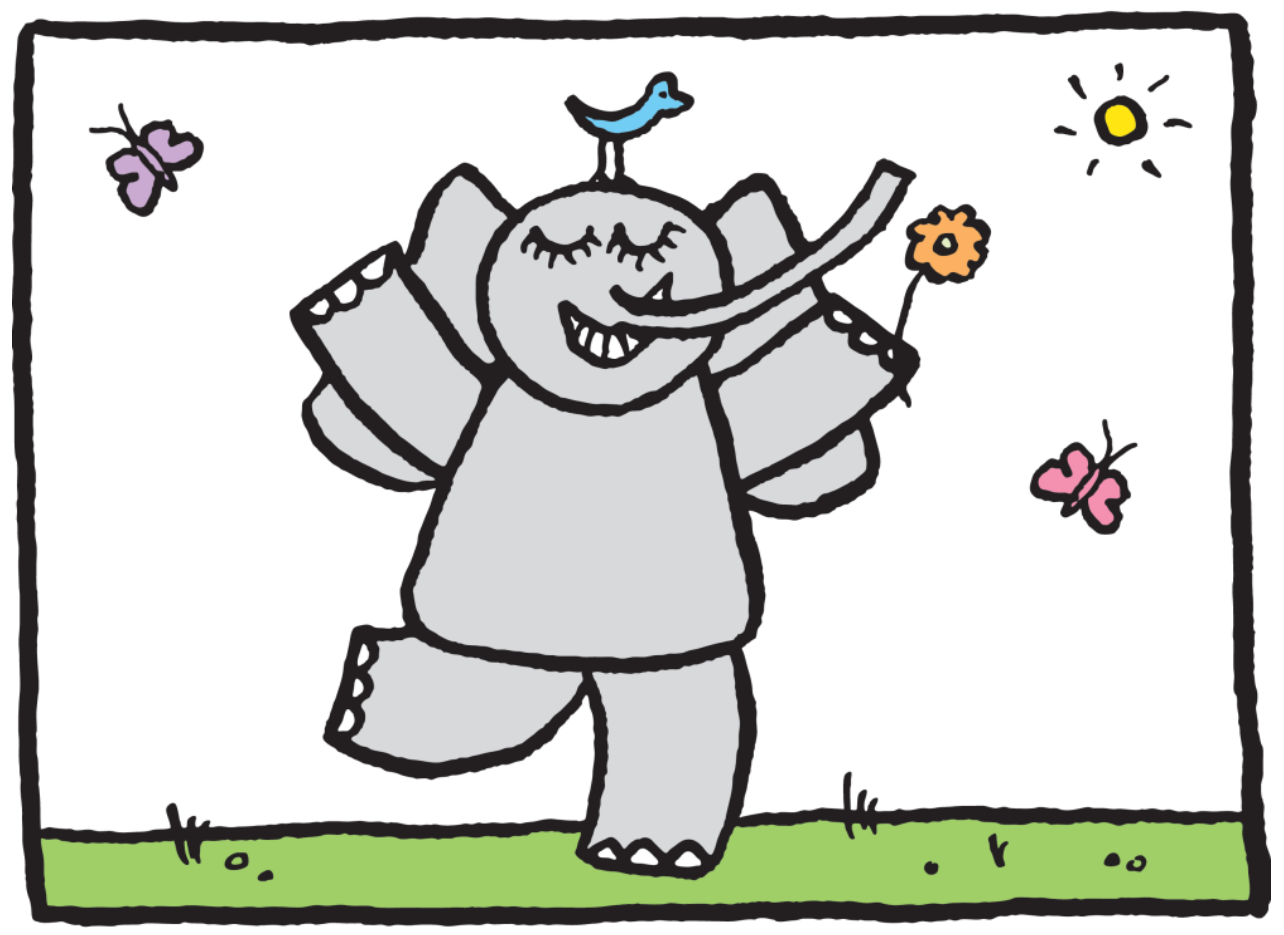

\section{References}


Aguilera, M., Arias, B., Wichers, M., Barrantes-Vidal, N., Moya, J., Villa, H., et al. (2009). Early adversity and 5HTT/BDNF genes: New evidence of gene-environment interactions on depressive symptoms in a general population. Psychol Med, 1-8.

Almagor, M., \& Ben-Porath, Y. S. (1989). The two-factor model of self-reported mood: A cross-cultural replication. Journal of Personality Assessment, 53(1), 10-21.

American Psychiatric Association. (2000). Diagnostic and Statistical Manual of Mental Disorders (4 ed.). Washington, DC.

Araya, R., Hu, K., Heron, J., Enoch, M. A., Evans, J., Lewis, G., et al. (2008). Effects of stressful life events, maternal depression and 5-HTTLPR genotype on emotional symptoms in pre-adolescent children. Am J Med Genet B Neuropsychiatr Genet.

Arntz, A., \& Wessel, I. (1996). Jeugd Trauma Vragenlijst (Dutch Version of the Childhood Trauma Questionnaire). Maastricht.

Baer, R. A. (2003). Mindfulness training as a clinical intervention: A conceptual and empirical review. Clinical Psychology: Science and Practice, 10(2), 125-143. doi: 10.1093/clipsy/bpg01

Baer, R. A. (2006). Mindfulness-based treatment approaches: Clinician's guide to evidence base and applications. Burlington, MA: Academic Press.

Baer, R. A., Smith, G. T., \& Allen, K. B. (2004). Assessment of mindfulness by self-report: The Kentucky Inventory of Mindfulness Skills. Assessment, 11(3), 191-206. doi: 10.1177/1073191104268029

Bagby, R. M., Ryder, A. G., Schuller, D. R., \& Marshall, M. B. (2004). The Hamilton Depression Rating Scale: Has the gold standard become a lead weight? American Journal of Psychiatry, 161(12), 2163-2177. doi: 10.1176/appi.ajp.161.12.2163

Ballesteros, J., Bobes, J., Bulbena, A., Luque, A., Dal-Ré, R., Ibarra, N., et al. (2007). Sensitivity to change, discriminative performance, and cutoff criteria to define remission for embedded short scales of the Hamilton Depression Rating Scale (HAMD). Journal of Affective Disorders, 102(1-3), 93-99.

Barge-Schaapveld, D., \& Nicolson, N. A. (2002). Effects of antidepressant treatment on the quality of daily life: An experience sampling study. Journal of Clinical Psychiatry, 63(6), 477-485.

Barge-Schaapveld, D. Q. C. M., Nicolson, N. A., Berkhof, J., \& deVries, M. W. (1999). Quality of life in depression: Daily life determinants and variability. Psychiatry Research, 88(3), 173-189. doi: 10.1016/S01651781(99)00081-5

Barnett, P. A., \& Gotlib, I. H. (1988). Psychosocial functioning and depression: Distinguishing among antecedents, concomitants, and consequences. Psychol Bull, 104(1), 97-126.

Barnhofer, T., Crane, C., Hargus, E., Amarasinghe, M., Winder, R., \& Williams, J. M. G. (2009). Mindfulness-based cognitive therapy as a treatment for chronic depression: A preliminary study. Behaviour Research and Therapy, 47(5), 366-373. doi: 10.1016/j.brat.2009.01.019

Barnhofer, T., Duggan, D., Crane, C., Hepburn, S., Fennell, M. J. V., \& Williams, J. M. G. (2007). Effects of meditation on frontal [alpha]-asymmetry in previously suicidal individuals. Neuroreport, 18(7), 709712. doi: 10.1097/WNR.0b013e3280d943cd

Baum, C., Kuyken, W., Bohus, M., Heidenreich, T., Michalak, J., \& Steil, R. (2010). The psychometric properties of the kentucky inventory of mindfulness skills in clinical populations. Assessment, 17(2), 220-229. doi: $10.1177 / 1073191109356525$

Baumeister, R. F., Bratslavsky, E., Finkenauer, C., \& Vohs, K. D. (2001). Bad is stronger than good. Review of General Psychology, 5(4), 323-370. doi: 10.1037//1089-2680.5.4.323

Baune, B. T., Hohoff, C., Berger, K., Neumann, A., Mortensen, S., Roehrs, T., et al. (2008). Association of the COMT $\mathrm{Val}^{158} \mathrm{Met}$ variant with antidepressant treatment response in major depression. Neuropsychopharmacology, 33, 924-932.

Beach, S. R., \& Amir, N. (2003). Is depression taxonic, dimensional, or both? J Abnorm Psychol, 112(2), 228-236.

Bearden, C. E., \& Freimer, N. B. (2006). Endophenotypes for psychiatric disorders: Ready for primetime? Trends in Genetics, 22(6), 306-313.

Bech, P. (2004). The impact of the SCL-90 on the validity of the DSM-IV neurotic or stress-related disorders. Acta Psychiatrica Scandinavica, 110, 161-162.

Beck, A. T. (2008). The evolution of the cognitive model of depression and its neurobiological correlates. American Journal of Psychiatry, 165(8), 969-977. doi: 10.1176/appi.ajp.2008.08050721

Beck, A. T., Rush, A. J., Shaw, B. F., \& Emergy, G. (1979). Cognitive therapy of depression. New York: Guilford Press.

Beevers, C. G., Rohde, P., Stice, E., \& Nolen-Hoeksema, S. (2007). Recovery from major depressive disorder among female adolescents: A prospective test of the Scar Hypothesis. Journal of Consulting and Clinical Psychology, 75(6), 888-900. 
Bergstrom, A., Jayatissa, M. N., Mork, A., \& Wiborg, O. (2008). Stress sensitivity and resilience in the chronic mild stress rat model of depression; an in situ hybridization study. Brain Res, 1196, 41-52.

Bernstein, D. P., Ahluvalia, T., Pogge, D., \& Handelsman, L. (1997). Validity of the Childhood Trauma Questionnaire in an adolescent psychiatric population. Journal of the American Academy of Child and Adolescent Psychiatry, 36(3), 340-348.

Bernstein, D. P., Fink, L., Handelsman, L., Foote, J., Lovejoy, M., Wenzel, K., et al. (1994). Initial reliability and validity of a new retrospective measure of child-abuse and neglect. American Journal of Psychiatry, $151(8), 1132-1136$.

Bhagwagar, Z., \& Cowen, P. J. (2008). 'It's not over when it's over': Persistent neurobiological abnormalities in recovered depressed patients. Psychological Medicine, 38, 307-313.

Bilder, R. M., Volavka, J., Lachman, H. M., \& Grace, A. A. (2004). The catechol-o-methyltransferase polymorphism: Relations to the tonic-phasic dopamine hypothesis and neuropsychiatric phenotypes. Neuropsychopharmacology, 29(11), 1943-1961.

Bondolfi, G., Jermann, F., Van der Linden, M., Gex-Fabry, M., Bizzini, L., Rouget, B. W., et al. (2010). Depression relapse prophylaxis with mindfulness-based cognitive therapy: Replication and extension in the swiss health care system. Journal of Affective Disorders, 122(3), 224-231. doi: 10.1016/j.jad.2009.07.007

Bouma, E. M., Ormel, J., Verhulst, F. C., \& Oldehinkel, A. J. (2008). Stressful life events and depressive problems in early adolescent boys and girls: The influence of parental depression, temperament and family environment. J Affect Disord, 105(1-3), 185-193.

Bradford-Hill, A., \& Proceedings of the Royal Society of Medicine, 295-300. (1965). The environment and disease: Association or causation? Proceedings of the Royal Society of Medicine, 58, 295-300.

Brown, K. W., \& Ryan, R. M. (2003). The benefits of being present: Mindfulness and its role in psychological wellbeing. Journal of Personality and Social Psychology, 84(4), 822-848. doi: 10.1037/0022-3514.84.4.82

Brunoni, A. R., Lopes, M., \& Fregni, F. (2008). A systematic review and meta-analysis of clinical studies on major depression and BDNF levels: Implications for the role of neuroplasticity in depression. Int $J$ Neuropsychopharmacol, 11(8), 1169-1180.

Burcusa, S. L., \& lacono, W. G. (2007). Risk for recurrence in depression. Clinical Psychology Review, 27(8), 959985. doi: 10.1016/j.cpr.2007.02.005

Burgdorf, J., \& Panksepp, J. (2006). The neurobiology of positive emotions. Neuroscience and Biobehavioral Reviews, 30, 173-187.

Burton, N. W., Pakenham, K. I., \& Brown, W. J. (2010). Feasibility and effectiveness of psychosocial resilience training: A pilot study of the ready program. Psychology, Health \& Medicine, 15(3), 266-277. doi: $10.1080 / 13548501003758710$

Bylsma, L. M., Morris, B. H., \& Rottenberg, J. (2008). A meta-analysis of emotional reactivity in major depressive disorder. Clinical Psychology Review, 28(4), 676-691. doi: 10.1016/j.cpr.2007.10.001

Bylsma, L. M., Taylor-Clift, A., \& Rottenberg, J. (2011). Emotional reactivity to daily events in major and minor depression. Journal of Abnormal Psychology, 120(1), 155-167. doi: 10.1037/a002166

Cacioppo, J. T., Gardner, W. L., \& Berntson, G. G. (1999). The affect system has parallel and integrative processing components: Form follows function. Journal of Personality and Social Psychology, 76(5), 839-855. doi: 10.1037/0022-3514.76.5.839

Cannon, T., \& Keller, M. C. (2005). Endophenotypes in the genetic analyses of mental disorders. Annual Review of Clinical Psychology, 2, 267-290.

Caspi, A., \& Moffitt, T. E. (2006). Gene-environment interactions in psychiatry: Joining forces with neuroscience. Neuroscience, 7, 583-590.

Caspi, A., Sugden, K., Moffitt, T. E., Taylor, A., Craig, I. W., Harrington, H., et al. (2003). Influence of life stress on depression: Moderation by a polymorphism in the 5-htt gene. Science, 301, 386-389.

Chen, Z.-Y., Jing, D., Bath, K. G., Ieraci, A., Khan, T., Siao, C.-J., et al. (2006). Genetic variant bdnf (Val ${ }^{66}$ Met) polymorphism alters anxiety-related behavior. Science, 314, 140-143.

Clark, L. A., Watson, D., \& Leeka, J. (1989). Diurnal variation in the positive affects. Motivation and Emotion, 13(3), 205-234. doi: 10.1007/bf00995536

Clayton, D., \& Hill, M. (1993). Wald tests. In D. Clayton \& M. Hills (Eds.), Statistical models in epidemiology (pp. 101-102). Oxford: Oxford Science Publications.

Coelho, H. F., Canter, P. H., \& Ernst, E. (2007). Mindfulness-based cognitive therapy: Evaluating current evidence and informing future research. Journal of Consulting and Clinical Psychology, 75(6), 1000-1005. doi: 10.1037/0022-006x.75.6.1000

Cohen, J. (1992). A power primer. Psychological Bulletin, 112, 155-159. 
Cohn, M. A., Fredrickson, B. L., Brown, S. L., Mikels, J. A., \& Conway, A. M. (2009). Happiness unpacked: Positive emotions increase life satisfaction by building resilience. Emotion, 9(3), 361-368. doi: $10.1037 / a 0015952$

Comijs, H. C., Beekman, A. T., Smit, F., Bremmer, M., van Tilburg, T., \& Deeg, D. J. (2007). Childhood adversity, recent life events and depression in late life. J Affect Disord, 103(1-3), 243-246.

Conway, M., Csank, P. A. R., Holm, S. L., \& Blake, C. K. (2000). On assessing individual differences in rumination on sadness. Journal of Personality Assessment, 75(3), 404-425.

Coyne, J. C. (1994). Self-reported distress: Analog or ersatz depression? Psychol Bull, 116(1), 29-45.

Craddock, N., Owen, M. J., \& O'Donovan, M. C. (2006). The catechol-o-methyl transferase (COMT) gene as a candidate for psychiatric phenotypes: Evidence and lessons. Molecular Psychiatry, 11(5), 446-458.

Csikszentmihalyi, M., \& Larson, R. (1987). Validity and reliability of the experience sampling method. Journal of Nervous and Mental Disease, 175(9), 526-536.

Cuijpers, P., \& Smit, F. (2004). Subthreshold depression as a risk indicator for major depressive disorder: A systematic review of prospective studies. Acta Psychiatrica Scandinavica, 109(5), 325-331. doi: 10.1111/j.1600-0447.2004.00301.x

Cuijpers, P., \& Smit, F. (2008). Has time come for broad-scale dissemination for prevention of depressive disorders? Acta Psychiatrica Scandinavica, 118(6), 419-420.

Dahabreh, I. J., \& Kent, D. M. (2011). Index event bias as an explanation for the paradoxes of recurrence risk research. Jama-Journal of the American Medical Association, 305(8), 822-823. doi: 10.1001/jama.2011.163

Danner, D. D., Snowdon, D. A., \& Friesen, W. V. (2001). Positive emotions in early life and longevity: Findings from the nun study. Journal of Personality and Social Psychology, 80(5), 804-813.

Dannlowski, U., Ohrmann, P., Bauer, J., Kugel, H., Arolt, V., Heindel, W., et al. (2007a). Amygdala reactivity to masked negative faces is associated with automatic judgmental bias in major depression: A 3 T FMRI study. J Psychiatry Neurosci, 32(6), 423-429.

Dannlowski, U., Ohrmann, P., Bauer, J., Kugel, H., Arolt, V., Heindel, W., et al. (2007b). Amygdala reactivity predicts automatic negative evaluations for facial emotions. Psychiatry Res, 154(1), 13-20.

Davidson, R. J. (1992). Emotion and affective style: Hemispheric substrates. Psychological Science, 3 (1), $39-43$.

Davidson, R. J., Ekman, P., Saron, C. D., Senulis, J. A., \& Friesen, W. V. (1990). Approach-withdrawal and cerebral asymmetry: Emotional expression and brain physiology: I. Journal of Personality and Social Psychology, 58(2), 330-341.

Davidson, R. J., \& Fox, N. A. (1982). Asymmetrical brain activity discriminates between positive and negative affective stimuli in human infants. Science, 218(4578), 1235-1237.

Davidson, R. J., Kabat-Zinn, J., Schumacher, J., Rosenkranz, M., Muller, D., Santorelli, S. F., et al. (2003). Alterations in brain and immune function produced by mindfulness meditation. Psychosomatic Medicine, 65(4), 564-570. doi: 10.1097/01.psy.0000077505.67574.e3

De Fruyt, F., Van Leeuwen, K., Bagby, R. M., Rolland, J. P., \& Rouillon, F. (2006). Assessing and interpreting personality change and continuity in patients treated for major depression. Psychol Assess, 18(1), 7180.

De Vries, M. (Ed.). (1992). The experience of psychopathology: Investigating mental disorders in their natural settings. Cambridge: Cambridge University Press.

Dekeyser, M., Raes, F., Leijssen, M., Leysen, S., \& Dewulf, D. (2008). Mindfulness skills and interpersonal behaviour. Personality and Individual Differences, 44, 1235-1245. doi: 10.1016/j.paid.2007.11.018

Delespaul, P. A. E. G. (1995). Assessing schizophrenia in daily life. The Experience Sampling Method. Maastricht: Maastricht University Press.

Delgado, L. C., Guerra, P., Perakakis, P., Vera, M. N., del Paso, G. R., \& Vila, J. (2010). Treating chronic worry: Psychological and physiological effects of a training programme based on mindfulness. Behaviour Research and Therapy, 48(9), 873-882. doi: 10.1016/j.brat.2010.05.012

Depue, R. A., Luciana, M., Arbisi, P., Collins, P., \& Leon, A. (1994). Dopamine and the structure of personality: Relation of agonist-induced dopamine activity to positive emotionality. Journal of Personality and Social Psychology, 67(3), 485-498.

Derogatis, L. R. (1994). SCL-90-R: Symptom Checklist-90-R. Administration, scoring and procedures manual. Minneapolis, MN: National Computer Systems.

Derogatis, L. R., Rickels, K., \& Rock, A. F. (1976). The SCL-90 and the MMPI: A step in the validation of a new selfreport scale. Br J Psychiatry, 128, 280-289.

Derom, C., Vlietinck, R., Thiery, E., Leroy, F., Fryns, J. P., \& Derom, R. (2002). The East Flanders Prospective Twin Survey (EFPTS). Twin Research, 5(5), 337-341. 
Derom, C. A., Vlietinck, R. F., Thiery, E. W., Leroy, F. O. G., Fryns, J. P., \& Derom, R. M. (2006). The East Flanders Prospective Twin Survey (EFPTS). Twin Research and Human Genetics, 9(6), 733-738.

DeVries, M. W. (Ed.). (1992). The experience of psychopathology: Investigating mental disorders in their natural settings. Cambridge: Cambridge university press.

Dichter, G. S., Tomarken, A. J., Freid, C. M., Addington, S., \& Shelton, R. C. (2005). Do venlafaxine XR and paroxetine equally influence negative and positive affect? Journal of Affective Disorders, 85(3), 333339.

Diener, E., \& Emmons, R. A. (1984). The independence of positive and negative affect. Journal of Personality and Social Psychology, 47(5), 1105-1117.

Dreher, J. C., Kohn, P., Kolachana, B., Weinberger, D. R., \& Berman, K. F. (2009). Variation in dopamine genes influences responsivity of the human reward system. Proceedings of the National Academy of Sciences of the United States of America, 106(2), 617-622. doi: 10.1073/pnas.0805517106

Duggan, C. F., Sham, P., Lee, A. S., \& Murray, R. M. (1991). Does recurrent depression lead to a change in neuroticism? Psychol Med, 21(4), 985-990.

Duman, R. S., \& Monteggia, L. M. (2006). A neurotrophic model for stress-related mood disorders. Biological Psychiatry, 59, 1116-1127.

Dunn, B. D., Dalgleish, T., Lawrence, A. D., Cusack, R., \& Ogilvie, A. D. (2004). Categorical and dimensional reports of experienced affect to emotion-inducing pictures in depression. Journal of Abnormal Psychology, 113(4), 654-666.

Easterlin, B., \& Cardena, E. (1998). Cognitive and emotional differences between short and long term vipassana meditators. Imagination, Cognition, \& Personality, 18(1), 69-81.

Eaves, L., Silberg, J., \& Erkanli, A. (2003). Resolving multiple epigenetic pathways to adolescent depression. J Child Psychol Psychiatry, 44(7), 1006-1014.

Eck, M. v., Nicolson, N. A., \& Berkhof, J. (1998). Effects of stressful daily events on mood states: Relationship to global perceived stress. Journal of Personality and Social Psychology, 75(6), 1572-1585.

Egan, M., Goldman, D., \& Weinberger, D. (2002). The human genome. American Journal of Psychiatry, $159(1), 12$.

Evans, S., Ferrando, S., Findler, M., Stowell, C., Smart, C., \& Haglin, D. (2008). Mindfulness-based cognitive therapy for generalized anxiety disorder. Journal of Anxiety Disorders, 22(4), 716-721. doi: 10.1016/j.janxdis.2007.07.005

Fanous, A., Gardner, C. O., Prescott, C. A., Cancro, R., \& Kendler, K. S. (2002). Neuroticism, major depression and gender: A population-based twin study. Psychological Medicine, 32(4), 719-728.

First, M. B., Spitzer, R. L., Gibbon, M., \& Williams, J. B. W. (1995). Structured Clinical Interview for DSM-IV Axis I Disorders. New York: Biometrics Research Department: New York State Psychiatric Institute.

First, M. B., Spitzer, R. L., Gibbon, M., \& Williams, J. B. W. (2002). Structured Clinical Interview for DSM-IV-TR Axis I Disorders, Research Version (November 2002 ed.). New York: Biometrics Research, New York State Psychiatric Institute.

Fish, E. W., Shahrokh, D., Bagot, R., Caldji, C., Bredy, T., Szyf, M., et al. (2004). Epigenetic programming of stress responses through variations in maternal care. Annals of the New York Academy of Science, 1036, 167-180.

Flett, G. L., Vredenburg, K., \& Krames, L. (1997). The continuity of depression in clinical and nonclinical samples. Psychol Bull, 121(3), 395-416.

Folkman, S., \& Moskowitz, J. T. (2000). Positive affect and the other side of coping. American Psychologist, 55(6), 647-654. doi: 10.1037//0003-066X.55.6.647

Fredrickson, B. L. (2001). The role of positive emotions in positive psychology - the broaden-and-build theory of positive emotions. American Psychologist, 56(3), 218-226. doi: 10.1037//0003-066X.56.3.218

Fredrickson, B. L. (2003). The value of positive emotions - the emerging science of positive psychology in coming to understand why it's good to feel good. American Scientist, 91(4), 330-335. doi: 10.1511/2003.4.330

Fredrickson, B. L. (2004). The broaden-and-build theory of positive emotions. Philosophical Transactions of the Royal Society of London Series B-Biological Sciences, 359(1449), 1367-1377. doi: 10.1098/rstb.2004.1512

Fredrickson, B. L., Cohn, M. A., Coffey, K. A., Pek, J., \& Finkel, S. M. (2008). Open hearts build lives: Positive emotions, induced through loving-kindness meditation, build consequential personal resources. Journal of Personality and Social Psychology, 95(5), 1045-1062. doi: 10.1037/a0013262

Fredrickson, B. L., \& Losada, M. F. (2005). Positive affect and the complex dynamics of human flourishing. American Psychologist, 60(7), 678-686. doi: 10.1037/0003-066x.60.7.678

Fredrickson, B. L., Mancuso, R. A., Branigan, C., \& Tugade, M. M. (2000). The undoing effect of positive emotions. Motivation and Emotion, 24(4), 237-258. 
Fredrickson, B. L., Tugade, M. M., Waugh, C. E., \& Larkin, G. R. (2003). What good are positive emotions in crises? A prospective study of resilience and emotions following the terrorist attacks on the united states on september 11th, 2001. Journal of Personality and Social Psychology, 84(2), 365-376. doi: 10.1037/0022-3514.84.2.365

Frijda, N. H., Kuipers, P., \& ter Schure, E. (1989). Relations among emotion, appraisal, and emotional action readiness. Journal of Personality and Social Psychology, 57(2), 212-228.

Gable, S. L., \& Haidt, J. (2005). What (and why) is positive psychology? Review of General Psychology, 9(2), 103110.

Galvan, A., Hare, T. A., Davidson, M., Spicer, J., Glover, G., \& Casey, B. J. (2005). The role of ventral frontostriatal circuitry in reward-based learning in humans. Journal of Neuroscience, 25(38), 8650-8656. doi: 10.1523/jneurosci.2431-05.2005

Ganzel, B., Casey, B. J., Glover, G., Voss, H. U., \& Temple, E. (2007). The aftermath of 9/11: Effect of intensity and recency of trauma on outcome. Emotion, 7(2), 227-238.

Ganzel, B. L., Kim, P., Glover, G. H., \& Temple, E. (2008). Resilience after 9/11: Multimodal neuroimaging evidence for stress-related change in the healthy adult brain. Neuroimage, 40(2), 788-795.

Garland, E. L., Fredrickson, B. L., Kring, A. M., Johnson, D. P., Meyer, P. S., \& Penn, D. L. (2010). Upward spirals of positive emotions counter downward spirals of negativity: Insights from the broaden-and-build theory and affective neuroscience on the treatment of emotion dysfunctions and deficits in psychopathology. Clinical Psychology Review, 30(7), 849-864. doi: 10.1016/j.cpr.2010.03.002

Gatt, J. M., Nemeroff, C. B., Dobson-Stone, C., Paul, R. H., Bryant, R. A., Schofield, P. R., et al. (2009). Interactions between bdnf val66met polymorphism and early life stress predict brain and arousal pathways to syndromal depression and anxiety. Mol Psychiatry.

Geschwind, N., Nicolson, N. A., Peeters, F., van Os, J., Barge-Schaapveld, D., \& Wichers, M. (2011a). Early improvement in positive rather than negative emotion predicts remission from depression after pharmacotherapy. European Neuropsychopharmacology, 21(3), 241-247. doi: 10.1016/j.euroneuro.2010.11.004

Geschwind, N., Peeters, F., Drukker, M., Van Os, J., \& Wichers, M. C. (2011b). Mindfulness training increases momentary positive emotions and reward experience in adults vulnerable to depression. A randomized controlled trial. Journal of Consulting and Clinical Psychology, 79(5), 618-628. doi: $10.1037 / \mathrm{a} 002459$

Geschwind, N., Peeters, F., Jacobs, N., Delespaul, P., Derom, C., Thiery, E., et al. (2010). Meeting risk with resilience: High daily life reward experience preserves mental health. Acta Psychiatrica Scandinavica, 122(2), 129-138. doi: 10.1111/j.1600-0447.2009.01525.x

Glaser, J.-P., van Os, J., Portegijs, P. J. M., \& Myin-Germeys, I. (2006). Childhood trauma and emotional reactivity to daily life stress in adult frequent attenders of general practitioners. Journal of Psychosomatic Research, 61(2), 229-236.

Godfrin, K. A., \& van Heeringen, C. (2010). The effects of mindfulness-based cognitive therapy on recurrence of depressive episodes, mental health and quality of life: A randomized controlled study. Behaviour Research and Therapy, 48(8), 738-746. doi: doi:10.1016/j.brat.2010.04.006

Goldapple, K., Segal, Z., Garson, C., Lau, M., Bieling, P., Kennedy, S., et al. (2004). Modulation of cortical-limbic pathways in major depression. Treatment-specific effects of cognitive behavior therapy. Archives of General Psychiatry, 61, 34-41.

Hamilton, M. (1960). A rating scale for depression. Journal of Neurology, Neurosurgery and Psychiatry, 23(1), 5662. doi: $10.1136 /$ jnnp.23.1.56

Hammen, C. (1991). Generation of stress in the course of unipolar depression. J Abnorm Psychol, 100(4), 555561.

Hammen, C. (2005). Stress and depression. Annual Review of Clinical Psychology, 1, 293-319.

Hankin, B. L., Fraley, R. C., Lahey, B. B., \& Waldman, I. D. (2005). Is depression best viewed as a continuum or discrete category? A taxometric analysis of childhood and adolescent depression in a populationbased sample. Journal of Abnormal Psychology, 114(1), 96-110.

Hargus, E., Crane, C., Barnhofer, T., \& Williams, J. M. G. (2010). Effects of mindfulness on meta-awareness and specificity of describing prodromal symptoms in suicidal depression. Emotion, 10(1), 34-42. doi: $10.1037 / \mathrm{a} 001682$

Harmer, C. J., de Bodinat, C., Dawson, G. R., Dourish, C. T., Waldenmaier, L., Adams, S., et al. (2010). Agomelatine facilitates positive versus negative affective processing in healthy volunteer models. Journal of Psychopharmacology. doi: 10.1177/0269881110376689

Hashimoto, K. (2007). Bdnf variant linked to anxiety-related behaviors. Bioessays, 29, 116-119. 
Hashimoto, K., Shimizu, E., \& lyo, M. (2004). Critical role of brain-derived neurotrophic factor in mood disorders. Brain Research Reviews, 45, 104-114.

Hasler, G., Drevets, W. C., Manji, H. K., \& Charney, D. S. (2004). Discovering endophenotypes for major depression. Neuropsychopharmacology, 29, 1765-1781.

Hayden, E. P., Dougherty, L. R., Maloney, B., Olino, T. M., Sheikh, H., Durbin, C. E., et al. (2008). Early-emerging cognitive vulnerability to depression and the serotonin transporter promoter region polymorphism. $J$ Affect Disord, 107(1-3), 227-230.

Heim, C., Newport, D. J., Heit, S., Graham, Y. P., Wilcox, M., Bonsall, R., et al. (2000). Pituitary-adrenal and autonomic responses to stress in women after sexual and physical abuse in childhood. JAMA, 284(5), 592-597.

Henkel, V., Seemuller, F., Obermeier, M., Adli, M., Bauer, M., Mundt, C., et al. (2009). Does early improvement triggered by antidepressants predict response/remission? Analysis of data from a naturalistic study on a large sample of inpatients with major depression. Journal of Affective Disorders, 115(3), 439-449. doi: 10.1016/j.jad.2008.10.011

Jacobs, N., Kenis, G., Peeters, F., Derom, C., Vlietinck, R., \& van Os, J. (2006). Stress-related negative affectivity and genetically altered serotonin transporter function: Evidence of synergism in shaping risk of depression. Archives of General Psychiatry, 63(9), 989-996. doi: 10.1001/archpsyc.63.9.989

Jacobs, N., Nicolson, N. A., Derom, C., Delespaul, P., van Os, J., \& Myin-Germeys, I. (2005). Electronic monitoring of salivary cortisol sampling compliance in daily life. Life Sciences, 76(21), 2431-2443. doi: 10.1016/j.Ifs.2004.10.045

Judd, L. L., Akiskal, H. S., Maser, J. D., Zeller, P. J., Endicott, J., Coryell, W., et al. (1998). Major depressive disorder: A prospective study of residual subthreshold depressive symptoms as predictor of rapid relapse. Journal of Affective Disorders, 50(2-3), 97-108. doi: 10.1016/S0165-0327(98)00138-4

Judd, L. L., Paulus, M. P., \& Zeller, P. (1999). The role of residual subthreshold depressive symptoms in early episode relapse in unipolar major depressive disorder. Archives of General Psychiatry, 56(8), 764-765.

Judd, L. L., Schettler, P. J., \& Akiskal, H. S. (2002). The prevalence, clinical relevance, and public health significance of subthreshold depressions. Psychiatric Clinics of North America, 25(4), 685-698. doi: 10.1016/S0193953X(02)00026-6

Kabat-Zinn, J. (1990). Full catastrophe living: The program of the stress reduction clinic at the university of massachusetts medical center. New York: Dell Publishing.

Kabat-Zinn, J. (2003). Mindfulness-based interventions in context: Past, present, and future. Clinical PsychologyScience and Practice, 10(2), 144-156. doi: 10.1093/clipsy/bpg016

Karson, C., Kleinman, J., Berman, K., Phelps, B., Wise, C., DeLisi, L., et al. (1983). An inverse correlation between spontaneous eye-blink rate and platelet monoamine oxidase activity. The British Journal of Psychiatry, 142(1), 43-46. doi: 10.1192/bjp.142.1.43

Kaufman, J., Yang, B.-Z., Douglas-Palumberi, H., Grasso, D., Lipschitz, D., Houshyar, S., et al. (2006). Brain-derived neurotrophic factor-5-httlpr gene interactions and environmental modifiers of depression in children. Biological Psychiatry, 59, 673-680.

Kazdin, A. E. (2007). Mediators and mechanisms of change in psychotherapy research. Annual Review of Clinical Psychology, 3(1), 1-27. doi: doi:10.1146/annurev.clinpsy.3.022806.091432

Kendler, K. S., \& Gardner, C. O. (1998). Boundaries of major depression: An evaluation of DSM-IV criteria. American Journal of Psychiatry, 155, 172-177.

Kendler, K. S., Kuhn, J. W., \& Prescott, C. A. (2004). Childhood sexual abuse, stressful life events and risk for major depression in women. Psychological Medicine, 34, 1475-1482.

Kendler, K. S., Thornton, L. M., \& Gardner, C. O. (2000). Stressful life events and previous episodes in the etiology of major depression in women: An evaluation of the "kindling" hypothesis. American Journal of Psychiatry, 157(1), 243-1251.

Kendler, K. S., Thornton, L. M., \& Gardner, C. O. (2001). Genetic risk, number of previous depressive episodes, and stressful life events in predicting onset of major depression. American Journal of Psychiatry, 158(4), 582-586.

Kendler, K. S., Thornton, L. M., \& Prescott, C. A. (2001). Gender differences in the rates of exposure to stressful life events and sensitivity to their depressogenic effects. American Journal of Psychiatry, 158(4), 587593.

Kenny, M. A., \& Williams, J. M. G. (2007). Treatment-resistant depressed patients show a good response to mindfulness-based cognitive therapy. Behaviour Research and Therapy, 45(3), 617-625. doi: 10.1016/j.brat.2006.04.008

Kessler, R. C. (1997). The effects of stressful life events on depression. Annual Review of Psychology, 48, 191-214. 
Killingsworth, M. A., \& Gilbert, D. T. (2010). A wandering mind is an unhappy mind. Science, 330(6006), 932. doi: 10.1126/science.1192439

Kingston, T., Dooley, B., Bates, A., Lawlor, E., \& Malone, K. (2007). Mindfulness-based cognitive therapy for residual depressive symptoms. Psychology and Psychotherapy-Theory Research and Practice, 80, 193203. doi: $10.1348 / 147608306 \times 116016$

Koenen, K. C., Moffitt, T. E., Poulton, R., Martin, J., \& Caspi, A. (2007). Early childhood factors associated with the development of post-traumatic stress disorder: Results from a longitudinal birth cohort. Psychological Medicine, 37(2), 181-192. doi: 10.1017/s0033291706009019

Kok, B. E., \& Fredrickson, B. L. (2010). Upward spirals of the heart: Autonomic flexibility, as indexed by vagal tone, reciprocally and prospectively predicts positive emotions and social connectedness. Biological Psychology, 85(3), 432-436. doi: 10.1016/j.biopsycho.2010.09.005

Kringelbach, M. L. (2005). The human orbitofrontal cortex: Linking reward to hedonic experience. Nature Reviews Neuroscience, 6, 691-702.

Kudielka, B. M., Broderick, J. E., \& Kirschbaum, C. (2003). Compliance with saliva sampling protocols: Electronic monitoring reveals invalid cortisol daytime profiles in noncompliant subjects. Psychosomatic Medicine, 65(2), 313-319. doi: 10.1097/01.psy.0000058374.50240.bf

Kumar, S., Feldman, G., \& Hayes, A. (2008). Changes in mindfulness and emotion regulation in an exposure-based cognitive therapy for depression. Cognitive Therapy and Research, 32(6), 734-744. doi: 10.1007/s10608-008-9190-1

Kuyken, W., Byford, S., Taylor, R. S., Watkins, E., Holden, E., White, K., et al. (2008). Mindfulness-based cognitive therapy to prevent relapse in recurrent depression. Journal of Consulting and Clinical Psychology, 76(6), 966-978. doi: 10.1037/a0013786

Kuyken, W., Watkins, E., Holden, E., White, K., Taylor, R. S., Byford, S., et al. (2010). How does mindfulness-based cognitive therapy work? Behaviour Research and Therapy, 48(11), 1105-1112. doi: 10.1016/j.brat.2010.08.003

Lang, U. E., Hellweg, R., Kalus, P., Bajbouj, M., Lenzen, K. P., Sander, T., et al. (2005). Association of a functional bdnf polymorphism and anxiety-related personality traits. Psychopharmacology, 180, 95-99.

Lataster, T., Wichers, M. C., Jacobs, N., Mengelers, R., Derom, C., Thiery, E., et al. (2009). Does reactivity to stress cosegregate with subclinical psychosis? A general population twin study. Acta Psychiatrica Scandinavica, 119(1), 45-53. doi: 10.1111/j.1600-0447.2008.01263.x

Laucht, M., Treutlein, J., Blomeyer, D., Buchmann, A. F., Schmid, B., Becker, K., et al. (2009). Interaction between the 5-httlpr serotonin transporter polymorphism and environmental adversity for mood and anxiety psychopathology: Evidence from a high-risk community sample of young adults. Int $J$ Neuropsychopharmacol, 1-11.

Leppänen, J. M. (2006). Emotional information processing in mood disorders: A review of behavioral and neuroimaging findings. Current Opinion in Psychiatry, 19(1), 34-39.

Leserman, J., Li, Z. M., Hu, Y. M. J. B., \& Drossman, D. A. (1998). How multiple types of stressors impact on health. Psychosomatic Medicine, 60(2), 175-181.

Lewinsohn, P. M., Steinmetz, J. L., Larson, D. W., \& Franklin, J. (1981). Depression-related cognitions: Antecedent or consequence? Journal of Abnormal Psychology, 90(3), 213-219. doi: 10.1037/0021-843x.90.3.213

Loos, R., Derom, C., Vlietinck, R., \& Derom, R. (1998). The East Flanders Prospective Twin Survey (Belgium): A population-based register. Twin Research, 1(4), 167-175.

Lutz, A., Slagter, H. A., Dunne, J. D., \& Davidson, R. J. (2008). Attention regulation and monitoring in meditation. Trends in Cognitive Sciences, 12(4), 163-169. doi: 10.1016/j.tics.2008.01.005

Lyubomirsky, S., King, L., \& Diener, E. (2005). The benefits of frequent positive affect: Does happiness lead to success? Psychological Bulletin, 131(6), 803-855. doi: 10.1037/0033-2909.131.6.803

Ma, S. H., \& Teasdale, J. D. (2004). Mindfulness-based cognitive therapy for depression: Replication and exploration of differential relapse prevention effects. Journal of Consulting and Clinical Psychology, 72(1), 31-40. doi: 10.1037/0022-006x.72.1.31

Malhi, G. S., \& Berk, M. (2007). Does dopamine dysfunction drive depression? Acta Psychiatrica Scandinavica, 115(s443), 116-124.

Mathew, K. L., Whitford, H. S., Kenny, M. A., \& Denson, L. A. (2010). The long-term effects of mindfulness-based cognitive therapy as a relapse prevention treatment for major depressive disorder. Behavioural and Cognitive Psychotherapy, 38(5), 561-576. doi: 10.1017/s135246581000010x

McFarland, B. R., \& Klein, D. N. (2009). Emotional reactivity in depression: Diminshed responsiveness to anticipated reward but not to anticipated punishment or to nonreward or avoidance. Depression and Anxiety, 26(2), 117-122. doi: 10.1002/da.20513 
Meyer, T. J., Miller, M. L., Metzger, R. L., \& Borkovec, T. D. (1990). Development and validation of the Penn State Worry Questionnaire. Behaviour Research and Therapy, 28, 487-495. doi: 10.1016/00057967(90)90135-6

Mill, J., \& Petronis, A. (2007). Molecular studies of major depressive disorder: The epigenetic perspective. Molecular Psychiatry, 12, 799-814.

Millan, M. J. (2009). Dual- and triple-acting agents for treating core and co-morbid symptoms of major depression: Novel concepts, new drugs. Neurotherapeutics, 6(1), 53-77.

Moffitt, T. E., Caspi, A., \& Rutter, M. (2005). Strategy for investigating interactions between measured genes and measured environments. Archives of General Psychiatry, 62, 473-481.

Monroe, S. M., \& Harkness, K. L. (2005). Life stress, the "kindling" hypothesis, and the recurrence of depression: Considerations from a life stress perspective. Psychological Review, 112(2), 417-445. doi: 10.1037/0033-295X.112.2.417

Morris, B. H., Bylsma, L. M., \& Rottenberg, J. (2009). Does emotion predict the course of major depressive disorder? A review of prospective studies. British Journal of Clinical Psychology, 48(3), 255-273. doi: 10.1348/014466508X396549

Mueller, T. I., Leon, A. C., Keller, M. B., Solomon, D. A., Endicott, J., Coryell, W., et al. (1999). Recurrence after recovery from major depressive disorder during 15 years of observational follow-up. American Journal of Psychiatry, 156(7), 1000-1006.

Myin-Germeys, I., \& van Os, J. (2007). Stress-reactivity in psychosis: Evidence for an affective pathway to psychosis. Clinical Psychology Review, 27(4), 409-424. doi: 10.1016/j.cpr.2006.09.005

Myin-Germeys, I., van Os, J., Schwartz, J. E., Stone, A. A., \& Delespaul, P. A. (2001). Emotional reactivity to daily life stress in psychosis. Archives of General Psychiatry, 58(12), 1137-1144. doi: 10.1001/archpsyc.58.12.1137

Nair, A., \& Vaidya, V. A. (2006). Cyclic amp response element binding protein and brain-derived neurotrophic factor: Molecules that modulate our mood? J. Biosci, 31, 423-434.

Nierenberg, A. A., Husain, M. M., Trivedi, M. H., Fava, M., Warden, D., Wisniewski, S. R., et al. (2010). Residual symptoms after remission of major depressive disorder with citalopram and risk of relapse: A STAR*D report. Psychological Medicine, 40(01), 41-50. doi: 10.1017/S0033291709006011

Nutt, D., Demyttenaere, K., Janka, Z., Aarre, T., Bourin, M., Canonico, P. L., et al. (2007). The other face of depression, reduced positive affect: The role of catecholamines in causation and cure. Journal of Psychopharmacology, 21(5), 461-471. doi: 10.1177/0269881106069938

Oberlander, T. F., Weinberg, J., Papsdorf, M., Grunau, R., Misri, S., \& Devlin, A. M. (2008). Prenatal exposure to maternal depression, neonatal methylation of human glucocorticoid receptor gene (NR3C1) and infant cortisol stress responses. Epigenetics, 3(2), 97-106.

Ormel, J., Oldehinkel, A. J., Nolen, W. A., \& Vollebergh, W. (2004a). Psychosocial disability before, during, and after a major depressive episode: A 3-wave population-based study of state, scar, and trait effects. Arch Gen Psychiatry, 61(4), 387-392.

Ormel, J., Oldehinkel, A. J., \& Vollebergh, W. (2004b). Vulnerability before, during, and after a major depressive episode: A 3-wave population-based study. Arch Gen Psychiatry, 61(10), 990-996.

Papakostas, G. I., Perlis, R. H., Scalia, M. J., Petersen, T. J., \& Fava, M. (2006). A meta-analysis of early sustained response rates between antidepressants and placebo for the treatment of major depressive disorder. Journal of Clinical Psychopharmacology, 26(1), 56-60. doi: 10.1097/01.jcp.0000195042.62724.76

Paykel, E. S. (1997). The interview for recent life events. Psychological Medicine, 27, 301-310.

Pearson, H. (2006). What is a gene? Nature, 441, 399-401.

Peeters, F., Berkhof, J., Delespaul, P., Rottenberg, J., \& Nicolson, N. A. (2006). Diurnal mood variation in major depressive disorder. Emotion, 6(3), 383-391. doi: 10.1037/1528-3542.6.3.383

Peeters, F., Nicolson, N. A., Berkhof, J., Delespaul, P., \& De Vries, M. (2003). Effects of daily events on mood states in major depressive disorder. Journal of Abnormal Psychology, 112(2), 203-211. doi: 10.1037/0021-843X.112.2.203

Pettit, J. W., Lewinsohn, P. M., \& Joiner, T. E., Jr. (2006). Propagation of major depressive disorder: Relationship between first episode symptoms and recurrence. Psychiatry Res, 141(3), 271-278.

Philibert, R. A., Sandhu, H., Hollenbeck, N., Gunter, T., Adams, W., \& Madan, A. (2008). The relationship of 5HTT (SLC6A4) methylation and genotype on mrna expression and liability to major depression and alcohol dependence in subjects from the iowa adoption studies. Am J Med Genet B Neuropsychiatr Genet, $147 B(5), 543-549$.

Pocock, S. J., Assmann, S. E., Enos, L. E., \& Kasten, L. E. (2002). Subgroup analysis, covariate adjustment and baseline comparisons in clinical trial reporting: Current practice and problems. Statistics in Medicine, 21, 2917-2930. doi: 10.1002/sim.1296 
Post, R. M. (1992). Transduction of psychosocial stress into the neurobiology of recurrent affective disorder. American Journal of Psychiatry, 149(8), 999-1010.

Posternak, M. A., \& Zimmerman, M. (2005). Is there a delay in the antidepressant effect? A meta-analysis. Journal of Clinical Psychiatry, 148-158.

Poulter, M. O., Du, L., Weaver, I. C., Palkovits, M., Faludi, G., Merali, Z., et al. (2008). Gaba(a) receptor promoter hypermethylation in suicide brain: Implications for the involvement of epigenetic processes. Biol Psychiatry.

Quitkin, F. M., Rabkin, J. G., Ross, D., \& Stewart, J. W. (1984). Identification of true drug response to antidepressants: Use of pattern analysis. Archives of General Psychiatry, 41(8), 782-786. doi: 10.1001/archpsyc.1984.01790190056007

Raes, F., Dewulf, D., Van Heeringen, C., \& Williams, J. M. G. (2009). Mindfulness and reduced cognitive reactivity to sad mood: Evidence from a correlational study and a non-randomized waiting list controlled study. Behaviour Research and Therapy, 47(7), 623-627. doi: 10.1016/j.brat.2009.03.007

Raes, F., Hermans, D., \& Eelen, P. (2003). De nederlandstalige versie van de Ruminative Response Scale (RRS-NL) en de Rumination On Sadness Scale (RSS-NL) [the Dutch version of the Ruminative Response Scale (RRS-NL) and the Rumination On Sadness Scale (RSS-NL)]. Gedragstherapie, 36, 97-104.

Ramel, W., Goldin, P. R., Carmona, P. E., \& McQuaid, J. R. (2004). The effects of mindfulness meditation on cognitive processes and affect in patients with past depression. Cognitive Therapy and Research, 28(4), 433-455. doi: 10.1023/B:COTR.0000045557.15923.96

Rawlings, N., Norbury, R., Cowen, P., \& Harmer, C. (2010). A single dose of mirtazapine modulates neural responses to emotional faces in healthy people. Psychopharmacology, 1-10. doi: 10.1007/s00213-0101983-8

Rohde, P., Lewinsohn, P. M., \& Seeley, J. R. (1990). Are people changed by the experience of having an episode of depression? A further test of the Scar Hypothesis. J Abnorm Psychol, 99(3), 264-271.

Rush, A. J., Gullion, C. M., Basco, M. R., Jarrett, R. B., \& Trivedi, M. H. (1996). The Inventory Of Depressive Symptomatology (IDS): Psychometric properties. Psychological Medicine, 26(03), 477-486. doi: doi:10.1017/S0033291700035558

Rush, A. J., Trivedi, M. H., Wisniewski, S. R., Nierenberg, A. A., Stewart, J. W., Warden, D., et al. (2006). Acute and longer-term outcomes in depressed outpatients requiring one or several treatment steps: A STAR*D report. American Journal of Psychiatry, 163(11), 1905-1917. doi: 10.1176/appi.ajp.163.11.1905

Schroevers, M. J., \& Brandsma, R. (2010). Is learning mindfulness associated with improved affect after mindfulness-based cognitive therapy? British Journal of Psychology, 101, 95-107. doi: $10.1348 / 000712609 \times 424195$

Schultz, W. (2002). Getting formal with dopamine and reward. Neuron, 36(2), 241-263.

Schwartz, R. M., Reynolds, C. F., III, Thase, M. E., Frank, E., Fasiczka, A. L., \& Haaga, D. A. F. (2002). Optimal and normal affect balance in psychotherapy of major depression: Evaluation of the balanced states of mind model. Behavioural and Cognitive Psychotherapy, 30(4), 439-450. doi: $10.1017 / \mathrm{s} 1352465802004058$

Segal, Z. V., Bieling, P., Young, T., MacQueen, G., Cooke, R., Martin, L., et al. (2010). Antidepressant monotherapy vs sequential pharmacotherapy and mindfulness-based cognitive therapy, or placebo, for relapse prophylaxis in recurrent depression. Archives of General Psychiatry, 67(12), 1256-1264. doi: 10.1001/archgenpsychiatry.2010.168

Segal, Z. V., Gemar, M., \& Williams, S. (1999). Differential cognitive response to a mood challenge following successful cognitive therapy or pharmacotherapy for unipolar depression. J Abnorm Psychol, 108(1), 3-10.

Segal, Z. V., Kennedy, S., Gemar, M., Hood, K., Pedersen, R., \& Buis, T. (2006). Cognitive reactivity to sad mood provocation and the prediction of depressive relapse. Arch Gen Psychiatry, 63(7), 749-755.

Segal, Z. V., Teasdale, J. D., Williams, J. M., \& Gemar, M. C. (2002a). The mindfulness-based cognitive therapy adherence scale: Inter-rater reliability, adherence to protocol and treatment distinctiveness. Clinical Psychology \& Psychotherapy, 9(2), 131-138. doi: 10.1002/cpp.320

Segal, Z. V., Williams, J. M., Teasdale, J. D., \& Gemar, M. (1996). A cognitive science perspective on kindling and episode sensitization in recurrent affective disorder. Psycholocal Medicine, 26, 371-380. doi: $10.1017 /$ S0033291700034760

Segal, Z. V., Williams, J. M. G., \& Teasdale, J. D. (2002b). Mindfulness-based cognitive therapy for depression: $A$ new approach to preventing relapse. New York: The Guilford Press.

Seligman, M., Ernst, R., Gillham, J., Reivich, K., \& Linkins, M. (2009). Positive education: Positive psychology and classroom interventions. Oxford Review of Education, 35(3), 293-311. doi: $10.1080 / 03054980902934563$ 
Seligman, M. E. P., \& Csikszentmihalyi, M. (2000). Positive psychology. An introduction. American Psychologist, 55(1), 5-14.

Seligman, M. E. P., Steen, T. A., \& Peterson, C. (2005). Positive psychology progress: Empirical validation of interventions. American Psychologist, Vol. 60, No. 65, 410-421. doi: 10.1037/0003-066X.60.5.410

Sen, S., Duman, R., \& Sanacora, G. (2008). Serum brain-derived neurotrophic factor, depression, and antidepressant medications: Meta-analyses and implications. Biol Psychiatry, 64(6), 527-532.

Shea, M. T., Leon, A. C., Mueller, T. I., Solomon, D. A., Warshaw, M. G., \& Keller, M. B. (1996). Does major depression result in lasting personality change? Am J Psychiatry, 153(11), 1404-1410.

Sheppard, L. C., \& Teasdale, J. D. (2004). How does dysfunctional thinking decrease during recovery from major depression? J Abnorm Psychol, 113(1), 64-71.

Slade, T. (2007). Taxometric investigation of depression: Evidence of consistent latent structure across clinical and community samples. Aust N Z J Psychiatry, 41(5), 403-410.

Sloan, D. M., Strauss, M. E., Quirk, S. W., \& Sajatovic, M. (1997). Subjective and expressive emotional responses in depression. Journal of Affective Disorders, 46, 135-141.

Snijders, T., \& Bosker, R. (1999). Multilevel analysis: An introduction to basis and advanced multilevel modeling. London: SAGE publications Ltd.

Solomon, A., Ruscio, J., Seeley, J. R., \& Lewinsohn, P. M. (2006). A taxometric investigation of unipolar depression in a large community sample. Psychol Med, 36(7), 973-985.

Stassen, H. H., Angst, J., Hell, D., Schayfetter, C., \& Szegedi, A. (2007). Is there a common resilience mechanism underlying antidepressant drug response? Evidence from 2848 patients. Journal of Clinical Psychiatry, 68(8), 1195-1205.

StataCorp. (2009). Stata statistical software: Release 11. College Station, TX: StataCorp LP.

Stroud, C. B., Davila, J., \& Moyer, A. (2008). The relationship between stress and depression in first onsets versus recurrences: A meta-analytic review. Journal of Abnormal Psychology, 117(1), 206-213.

Szegedi, A., Jansen, W. T., van Willigenburg, A. P. P., van der Meulen, E., Stassen, H. H., \& Thase, M. E. (2009). Early improvement in the first 2 weeks as a predictor of treatment outcome in patients with major depressive disorder: A meta-analysis including 6562 patients. Journal of Clinical Psychiatry, 70(3), 344353.

Szegedi, A., Rujescu, D., Tadic, A., Müller, M. J., Kohnen, R., Stassen, H. H., et al. (2005). The catechol-omethyltransferase $\mathrm{Val}{ }^{108 / 158}$ Met polymorphism affects short-term treatment response to mirtazapine, but not to paroxetine in major depression. The Pharmacogenomics Journal, 5, 49-53.

Tadic, A., Helmreich, I., Mergl, R., Hautzinger, M., Kohnen, R., Henkel, V., et al. (2010). Early improvement is a predictor of treatment outcome in patients with mild major, minor or subsyndromal depression. Journal of Affective Disorders, 120(1-3), 86-93.

Tang, T. Z., Luborsky, L., \& Andrusyna, T. (2002). Sudden gains in recovering from depression: Are they also found in psychotherapies other than cognitive-behavioral therapy? Journal of Consulting and Clinical Psychology, 70(2), 444-447. doi: 10.1037//0022-006x.70.2.444

Teasdale, J. D., Moore, R. G., Hayhurst, H., Pope, M., Williams, S., \& Segal, Z. V. (2002). Metacognitive awareness and prevention of relapse in depression: Empirical evidence. J Consult Clin Psychol, 70(2), 275-287.

Teasdale, J. D., Segal, Z. V., \& Williams, J. M. G. (1995). How does cognitive therapy prevent depressive relapse and why should attentional control (mindfulness) training help. Behaviour Research and Therapy, 33(1), 25-39. doi: 10.1016/0005-7967(94)E0011-7

Teasdale, J. D., Segal, Z. V., Williams, J. M. G., Ridgeway, V. A., Soulsby, J. M., \& Lau, M. A. (2000). Prevention of relapse/recurrence in major depression by mindfulness-based cognitive therapy. Journal of Consulting and Clinical Psychology, 68(4), 615-623. doi: 10.1037//0022-006X.68.4.615

The European Network of Schizophrenia Networks for the Study of Gene-Environment Interactions (EU-GEI). (2008). Schizophrenia aetiology: Do gene-environment interactions hold the key? Schizophrenia Research, 102, 21-26.

Tomarken, A. J., Dichter, G. S., Freid, C., Addington, S., \& Shelton, R. C. (2004). Assessing the effects of bupropion sr on mood dimensions of depression. Journal of Affective Disorders, 78(3), 235-241.

Tooby, J., \& Cosmides, L. (1990). The past explains the present: Emotional adaptations and the structure of ancestral environments. Ethology and Sociobiology, 11(4-5), 375-424.

Tugade, M. M., \& Fredrickson, B. L. (2004). Resilient individuals use positive emotions to bounce back from negative emotional experiences. Journal of Personality and Social Psychology, 86(2), 320-333. doi: 10.1037/0022-3514.86.2.320

Tugade, M. M., Fredrickson, B. L., \& Barrett, L. F. (2004). Psychological resilience and positive emotional granularity: Examining the benefits of positive emotions on coping and health. Journal of Personality, 72(6), 1161-1190. doi: 10.1111/j.1467-6494.2004.00294.x 
Van Os, J., \& Jones, P. B. (1999). Early risk factors and adult person-environment relationships in affective disorder. Psychological Medicine, 29, 1055-1067.

Van Praag, H. M., De Kloet, R., \& Van Os, J. (2004a). Life events and depression: Is there a causal connection. In H. M. Van Praag, R. De Kloet \& J. Van Os (Eds.), Stress, the brain, and depression (pp. 38-58). Cambridge: University Press.

Van Praag, H. M., De Kloet, R., \& Van Os, J. (2004b). Stress, the brain, and depression. Cambridge: University Press.

Van Schaik, D. J. F., Klijn, A. F. J., van Hout, H. P. J., van Marwijk, H. W. J., Beekman, A. T. F., de Haan, M., et al. (2004). Patients' preferences in the treatment of depressive disorder in primary care. General Hospital Psychiatry, 26(3), 184-189. doi: 10.1016/j.genhosppsych.2003.12.001

Verhagen, M., van der Meij, A., van Deurzen, P. A., Janzing, J. G., Arias-Vasquez, A., Buitelaar, J. K., et al. (2008). Meta-analysis of the BDNF Val ${ }^{66}$ Met polymorphism in major depressive disorder: Effects of gender and ethnicity. Mol Psychiatry.

Waite, P. J., \& Richardson, G. E. (2004). Determining the efficacy of resiliency training in the work site. Journal of Allied Health, 33(3), 178-183.

Watson, D., Clark, L. A., \& Carey, G. (1988). Positive and negative affectivity and their relation to anxiety and depressive disorders. Journal of Abnormal Psychology, 97(3), 346-353.

Watson, D., \& Tellegen, A. (1985). Toward a consensual structure of mood. Psychological Bulletin, 98, 219-235.

Watson, D., Wiese, D., Vaidya, J., \& Tellegen, A. (1999). The two general activation systems of affect: Structural findings, evolutionary considerations, and psychobiological evidence. Journal of Personality and Social Psychology, 76(5), 820-838.

Wichers, M. C., Aguilera, M., Kenis, G., Krabbendam, L., Myin-Germeys, I., Jacobs, N., et al. (2008a). The catecholo-methyl transferase $\mathrm{Val}^{158}$ Met polymorphism and experience of reward in the flow of daily life. Neuropsychopharmacology, 33(13), 3030-3036. doi: 10.1038/sj.npp.1301520

Wichers, M. C., Barge-Schaapveld, D. Q. C. M., Nicolson, N. A., Peeters, F., de Vries, M., Mengelers, R., et al. (2009a). Reduced stress-sensitivity or increased reward experience: The psychological mechanism of response to antidepressant medication. Neuropsychopharmacology, 34, 923-931. doi: 10.1038/npp.2008.1066

Wichers, M. C., Geschwind, N., Jacobs, N., Kenis, G., Peeters, F., Derom, C., et al. (2009b). Transition from stress sensitivity to a depressive state: Longitudinal twin study. The British Journal of Psychiatry, 195(6), 498503. doi: 10.1192/bjp.bp.108.056853

Wichers, M. C., Kenis, G., Jacobs, N., Myin-Germeys, I., Schruers, K., Mengelers, R., et al. (2008b). The psychology of psychiatric genetics: Evidence that positive emotions in females moderate genetic sensitivity to social stress associated with the BDNF Val ${ }^{66} \mathrm{Met}$ polymorphism. Journal of Abnormal Psychology, 117(3), 699-704. doi: 10.1037/a0012909

Wichers, M. C., Myin-Germeys, I., Jacobs, N., Kenis, G., Derom, C., Vlietinck, R., et al. (2008c). Susceptibility to depression expressed as alterations in cortisol day curve: A cross-twin, cross-trait study. Psychosomatic Medicine, 70(3), 314-318.

Wichers, M. C., Myin-Germeys, I., Jacobs, N., Peeters, F., Kenis, G., Derom, C., et al. (2007a). Evidence that moment-to-moment variation in positive emotions buffer genetic risk for depression: A momentary assessment twin study. Acta Psychiatrica Scandinavica, 115(6), 451-457. doi: 10.1111/j.16000447.2006.00924.x

Wichers, M. C., Myin-Germeys, I., Jacobs, N., Peeters, F., Kenis, G., Derom, C., et al. (2007b). Genetic risk of depression and stress-induced negative affect in daily life. British Journal of Psychiatry, 191, 218-223. doi: 10.1192/bjp.bp.106.032201

Wichers, M. C., Peeters, F., Geschwind, N., Jacobs, N., Simons, C. J. P., Derom, C., et al. (2010). Unveiling patterns of affective responses in daily life may improve outcome prediction in depression: A momentary assessment study. Journal of Affective Disorders, 124(1), 191-195. doi: 10.1016/j.jad.2009.11.010

Wichers, M. C., Schrijvers, D., Geschwind, N., Jacobs, N., Myin-Germeys, I., Thiery, E., et al. (2009c). Mechanisms of gene-environment interactions in depression: Evidence that genes potentiate multiple sources of adversity. Psychological Medicine, 39, 1077-1086.

Williams, J. M. G. (2008). Mindfulness, depression and modes of mind. Cognitive Therapy and Research, 32(6), 721-733. doi: 10.1007/s10608-008-9204-z

World Health Organization. (2001). Burden of mental and behavioral disorders. The World Health Report 2001 Mental Health: New understanding, new hope (pp. 19-45).

Yoshida, K., Higuchi, H., Takahashi, H., Kamata, M., Sato, K., Inoue, K., et al. (2008). Influence of the tyrosine hydroxylase val81met polymorphism and catechol-o-methyltransferase Val ${ }^{158}$ Met polymorphism on 
the antidepressant effect of milnacipran. Human Psychopharmacology: Clinical and Experimental, 23, 121-128.

Zautra, A. J., Johnson, L. M., \& Davis, M. C. (2005). Positive affect as a source of resilience for women in chronic pain. Journal of Consulting and Clinical Psychology, 73(2), 212-220. doi: 10.1037/0022-006X.73.2.212

Zeiss, A. M., \& Lewinsohn, P. M. (1988). Enduring deficits after remissions of depression: A test of the scar hypothesis. Behav Res Ther, 26(2), 151-158.

Zobel, A. W., Nickel, T., Sonntag, A., Uhr, M., Holsboer, F., \& Ising, M. (2001). Cortisol response in the combined dexamethasone/crh test as predictor of relapse in patients with remitted depression. A prospective study. J Psychiatr Res, 35(2), 83-94. 



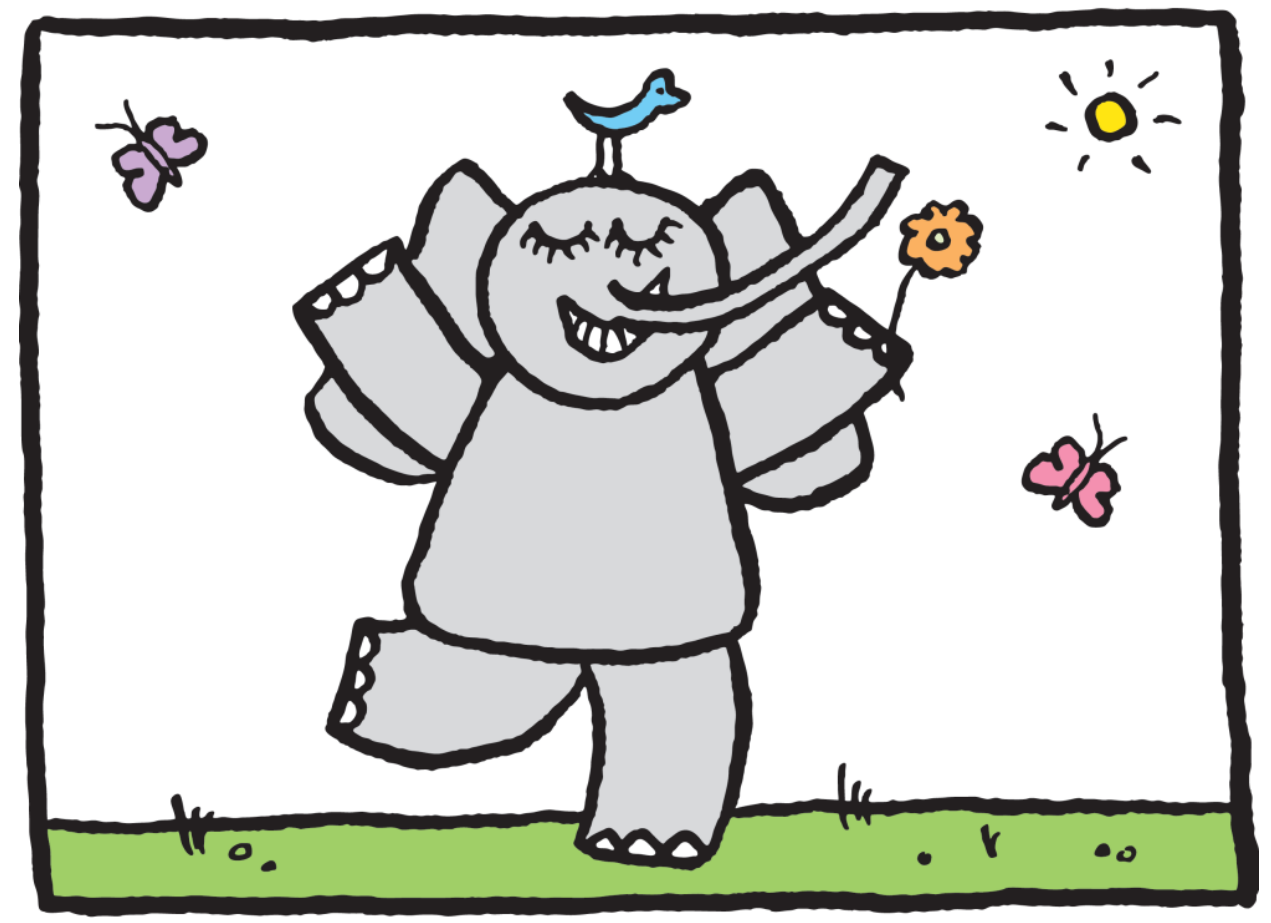

\section{Thanks to...}


Allereerst dank aan mijn geweldig team van (co)promotoren. Marieke, heel erg bedankt dat mijn dagelijkse begeleider bent geweest! Ik kon altijd jouw kamer binnenlopen met dringende vragen, en met een nieuwe injectie van onverzettelijk optimisme en inspiratie weer vertrekken. Ik zal jou en ons onlangs opgerichte meditatieclubje missen! Frenk, dank je wel voor je klinische input, je advies en je betrokkenheid. Ik keek altijd uit naar je commentaar op nieuwe manuscripten, waarin je suggesties of kritiek afwisselde met kleurrijke complimenten. Jim, ondanks je drukke agenda kon ik altijd rekenen op een snelle reactie en steun waar het nodig was. Ik voel me vereerd dat ik met jullie mocht samenwerken en van jullie mocht leren en hoop dat ook in toekomst nog te kunnen blijven doen.

Mijn paranimfen.... Rufa, je hebt me gered toen ik bijna verdronk in aanmeldingen voor het MindMaastricht onderzoek! Ik vond het heel erg fijn om met jou samen te werken, te sporten en vrienden te worden. Nicole, heel gezellig dat we de laatste maanden een kamer (en de dagelijkse highs en lows van het onderzoek doen) hebben gedeeld. Ik zal je luisterend oor missen (net als de niet nalatende stroom van importkoekjes en chocola)! Ook bedankt voor het babysitten - goed om Jamie in jouw ervaren handen te weten...

Greet Kellens en Marga Schnitzeler, heel erg bedankt voor jullie hulp bij de verzameling van de follow-up data. Karlijn Gielen, jouw zelfbeschilderde koekjesboord vormt nog steeds een hele decoratieve achtergrond voor bovengenoemde koekjes. Ook aan jou dank voor je hulp bij de dataverzameling, net als Fritz Renner. Rob Vincken, Franca Warmenhoven en de andere SeeTrue Mindfulness trainers, bedankt voor de prettige samenwerking en de flexibele opstelling als er (in verband met de randomizatie) vaak nog op het laatste minuut deelnemers voor een mindfulness training geplaatst moesten worden. Anja Karst en Marionne Wolfis, bedankt voor jullie bereidheid om mee te werken aan het onderzoek. Ook aan de andere therapeuten van de RIAGG: dank jullie wel voor het doorverwijzen van geïnteresseerde cliënten. Frits Vissers, dank voor de interessante gesprekken over flow en heartcoherentie en je training met de EmWave.

Ook de deelnemers van het MindMaastricht onderzoek wil ik van harte danken voor hun inzet en hun bereidheid om 2 weken lang met een piepend horloge rond te lopen en hun ervaringen met ons te delen.

Nele Jacobs en Claudia Lothman, het was altijd leuk om samen op congres te gaan en cultuur en etensgelegenheden te verkennen. Speedy Dina en Margreet, leuk dat we niet alleen samen de Research Master gedaan hebben maar voor de PhD op dezelfde afdeling zijn belandt! Odette, Mari, Rebecca, Petra, Margriet, Steffi, Johan, en Tineke - leuk dat jullie er waren. Lunchers, 
bedankt voor de gezellige lunchpauzes! Martine, tot snel weer op de spinningfiets...

Anne Fett, bedankt voor je vriendschap, gezellige etentjes in Maastricht en cultuur, muziek en wilde dansnachten in London. Valentina, thanks for your music and film suggestions, good conversations and shared experiences in the big city. Hope to see you again soon! Frühling, thank you for being my host in London! And also for saving me from forced physical inactivity when it turned out that one needed a British bank account in order to become a member at the gym. Zarsh, Lucy (fellow early veggie moral twin-separated-at-birth), and Georgina, thank you for shared lunch breaks and witty or funny conversations. Marisa, bloodypump is not the same without you.

Marjan, bedankt voor de gezellige praatjes over paarden, konijnen en andere dieren, en voor je advies op statistisch gebied. Wolfgang, jij ook van harte bedankt voor je advies, de powerberekeningen, en je bewonderenswaardige kennis van de statistiek. Ron, dank je wel voor je altijd snelle hulp als de computer weer eens niet mee wilde werken!!! Nancy, leuk om je hier weer tegen te komen na afloop van het RM programma - het was fijn om met je samen te werken. Inez, je daadkracht, gedrevenheid, en enthousiasme zijn bewonderenswaardig. Dank ook voor het voorzitten van de leescommissie. Gunter, Ruud en Bart, dank voor de samenwerking wat betreft de genetische aspecten (en Bart ook voor het uitlenen van de babykleertjes, en voor de luieroefensessie). Koen, dank voor je poging tot introductie in de Vlaamse cultuur, ik hoop dat je gelijk gaat hebben met het gat en de boter... Truda, Frieda, Philippe, en Deborah, bedankt voor de hulp met de dagboekjes of met organisatorische aspecten van de studie. Trees, Leni, Jolanda, Ine, Wendy, ook jullie bedankt voor jullie steun (en Leni ook voor de leuke kaartjes, versieringen, en lekkernijen).

Anita Jansen en Anne Roefs, in jullie eetgroep heb ik veel geleerd over onderzoek - fijn dat we nog altijd af en toe contact hebben!

Gute Freunde sind selten und wichtig - danke dass es euch gibt. Meine liebe Hannah, Christian und Najima - auf viele weitere schönen Wochenenden im Spessart, und Zeit füreinander. Anne and Nathi, I wish we could see each other more often. Looking forward to the next time, with little Shoshana! Elissa, thanks for your friendship, and the bunny sitting, Alex too. Anna and Thomas, thank you for many delicious meals and fun evenings. Please come back soon! Martijn, ik meld me alvast aan voor en parachutesprong met jou. Selma en Hugo, jullie zullen altijd welkom zijn in ons huis (welk huis het ook is ;-)). Linda, ik hoop dat we 


\section{Acknowledgements}

blijven afspreken met Stijn en Jamie. Anouk, het was leuk om je vaker te zien de afgelopen tijd. Niet te ver weg verhuizen, hé?

Sonja en Gerus, een weekendje Den Haag is altijd ontspanning puur... bedankt! Mum and Dad, thanks for always being there, supporting me and believing in me. A big thank you to Granny Weatherwax, too! Sis Kit, schön dass es Dich gibt - und auch Uncle Greg. Special thanks also to Eilis, Tony, Wendy and Neil.

Sibren, love of my life - I am looking forward to many more wonderful years to come. Jamie, so glad you joined us... I enjoy every moment with you, and hope you will always be as cheerful and happy as you are now! 


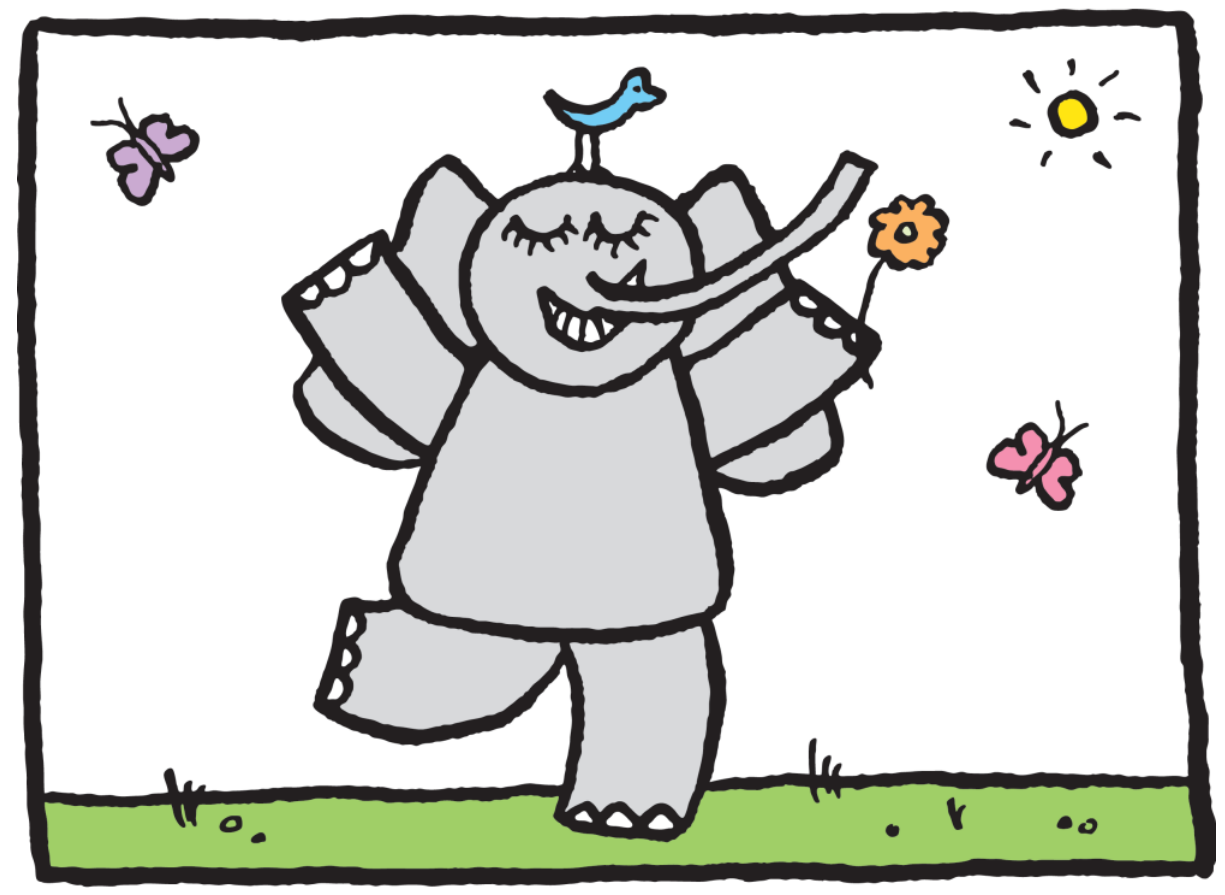

\section{C.V. and Publications}




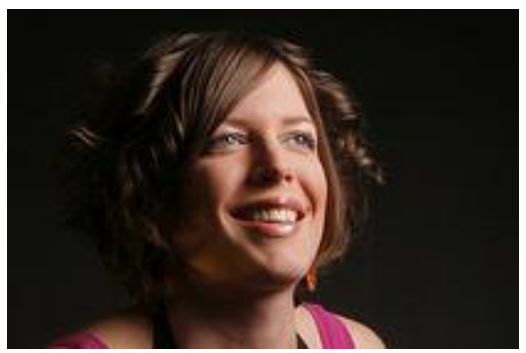

Nicole Geschwind was born on September $7^{\text {th }}$, 1979 in Bremen, Germany. She graduated from secondary school (Gymnasium Liebfrauenschule, Cologne) in 1998. From 2002 on, she studied Psychology at Maastricht University, the Netherlands. In 2005, she obtained her Bachelor's degree in Cognitive

Psychology (cum laude). During the two-year Research Master's program, she specialized in Psychopathology, and obtained her Master's Degree in Cognitive Neuroscience, Neuropsychology, and Psychopathology in 2007 (cum laude). During her studies, Nicole frequently worked as a research assistant, resulting in several (co)publications on eating behaviour and addiction. From 2007 until 2011, she worked on her PhD project at the Department of Psychiatry and Psychology, Maastricht University. During this time, she spent 6 months at the Institute of Psychiatry, King's College London, UK. In March 2011, she gave birth to her son Jamie. From January 2012 on, she will work as a post-doc at the University of Leuven, Belgium.

\section{PUBLICATIONS}

Fett, A.-K., Lattimore, P., Roefs, A., Geschwind, N., \& Jansen, A. (2009). Food cue exposure and body image satisfaction: The moderating role of $\mathrm{BMI}$ and dietary restraint. Body Image, 6(1), 14-18.

Geschwind, N., Nicolson, N. A., Peeters, F., van Os, J., Barge-Schaapveld, D., \& Wichers, M. (2011a). Early improvement in positive rather than negative emotion predicts remission from depression after pharmacotherapy. European Neuropsychopharmacology, 21(3), 241-247. doi: 10.1016/j.euroneuro.2010.11.004

Geschwind, N., Peeters, F., Drukker, M., Van Os, J., \& Wichers, M. (2011b). Mindfulness training increases momentary positive emotions and reward experience in adults vulnerable to depression. A randomized controlled trial. Journal of Consulting and Clinical Psychology, 79(5), 618-628. doi: 10.1037/a002459

Geschwind, N., Peeters, F., Jacobs, N., Delespaul, P., Derom, C., Thiery, E., . . . Wichers, M. (2010). Meeting risk with resilience: High daily-life reward experience preserves mental health. Acta Psychiatrica Scandinavica, 122(2), 129-138. doi: 10.1111/j.1600-0447.2009.01525.x 
Geschwind, N., Roefs, A., Lattimore, P., Fett, A.-K., \& Jansen, A. (2008). Dietary restraint moderates the effects of food exposure on women's body and weight satisfaction. Appetite, 51(3), 735-738.

Geschwind, N., van Os, J., Peeters, F., \& Wichers, M. (2009). The role of affective processing in vulnerability to and resilience against depression. In C. M. Pariante, R. M. Nesse, D. Nutt \& L. Wolpert (Eds.), Understanding depression: A translational approach (pp. 181-192). NY: New York: Oxford University Press Inc.

Guerrieri, R., Nederkoorn, C., Stankiewicz, K., Alberts, H., Geschwind, N., Martijn, C., \& Jansen, A. (2007). The influence of trait and induced state impulsivity on food intake in normal-weight healthy women. Appetite, 49(1), 66-73. doi: 10.1016/j.appet.2006.11.008

Havermans, R. C., Geschwind, N., Filla, S., Nederkoorn, C., \& Jansen, A. (2009). Sensory-specific satiety is unaffected by manipulations of flavour intensity. Physiology and Behavior, 97, 327-333.

Thewissen, R., Havermans, R. C., Geschwind, N., van den Hout, M., \& Jansen, A. (2007). Pavlovian conditioning of an approach bias in low-dependent smokers. Psychopharmacology, 194(1), 33-39. doi: 10.1007/s00213-0070819-7

Wichers, M., Geschwind, N., Jacobs, N., Kenis, G., Peeters, F., Derom, C. , . . van Os, J. (2009a). Transition from stress sensitivity to a depressive state: Longitudinal twin study. The British Journal of Psychiatry, 195(6), 498-503. doi: 10.1192/bjp.bp.108.056853

Wichers, M., Geschwind, N., van Os, J., \& Peeters, F. (2010a). Scars in depression: Is a conceptual shift necessary to solve the puzzle? Psychological Medicine, 40(3), 359-365. doi: 10.1017/s0033291709990420

Wichers, M., Peeters, F., Geschwind, N., Jacobs, N., Simons, C. J. P., Derom, C., . . van Os, J. (2010b). Unveiling patterns of affective responses in daily life may improve outcome prediction in depression: A momentary assessment study. Journal of Affective Disorders, 124(1), 191-195. doi: 10.1016/j.jad.2009.11.010

Wichers, M., Schrijvers, D., Geschwind, N., Jacobs, N., Myin-Germeys, I., Thiery, E., et al. (2009b). Mechanisms of gene-environment interactions in depression: Evidence that genes potentiate multiple sources of adversity. Psychological Medicine, 39, 1077-1086. 


\section{SUBMITTED}

Cramer, A. O. J., Sluis, S. v. d., Noordhof, A., Wichers, M., Geschwind, N., Aggen, S. H., ... Borsboom, D. (invited paper). Dimensions of normal personality as networks in search of equilibrium: You can't like parties if you don't like people. European Journal of Personality

Geschwind, N., Peeters, F., Van Os, J., \& Wichers, M. (submitted). Mindfulnessbased cognitive therapy reduces residual depressive symptoms irrespective of number of prior depressive episodes. A randomized controlled trial.

Rutten, B. P., Hammels, C., Geschwind, N., Lothmann, C., Pishva, E., Kenis, G., . . Wichers, M. (invited paper). Resilience in Mental Health: Linking Psychological and Neurobiological Perspectives. Acta Psychiatrica Scandinavica.

\section{PRESENTATIONS}

Oral presentation at the Invitational Symposium on Mindfulness Research, in Amsterdam, the Netherlands, November 2011. Effects of MindfulnessBased Cognitive Therapy on the experience of positive emotions in daily life: A Randomized Controlled Trial.

Oral presentation at the Emotions Congres in Tilburg, the Netherlands, October 2011. Effects of Mindfulness-Based Cognitive Therapy on the experience of positive emotions in daily life: A Randomized Controlled Trial.

Invited lecture in the seminar series 'New Trends in Human Genetics' at the Institute of Human Genetics, Bonn University, Germany, November 2010. Topic: Gene-environment interaction and momentary assessment of reactive phenotypes in psychiatry.

Invited lecture at the Institute of Psychiatry, King's College London, UK, June 2010. Topic: Recovery from and resilience against depression: The role of positive emotions and experiences of reward in the flow of daily life.

Poster presentation at the Psychiatric Genetics congress in Athens, Greece, October 2010. GxE in daily-life Reward Experience: COMT Val ${ }^{158} \mathrm{Met}$ interacts with pleasant events.

Hot Topic oral presentation at the International Society for Research on Emotion (ISRE) congress in Leuven, Belgium, August 2009. In women at risk for low mood, daily-life reward experience protects against future affective symptoms: A longitudinal twin study. 
Poster presentation at the first world congress of the International Positive Psychology Association (IPPA), Philadelphia, USA, June 2009. In women at risk for low mood, daily-life reward experience protects against future affective symptoms: A longitudinal twin study.

Poster Award Oral Presentation at the Mental Health and Neuroscience scientific meeting, March 2009. In women, daily-life reward experience protects against developing stress-induced affective symptoms: A longitudinal twin study.

Poster Award Oral Presentation at the Association of European Psychiatrists' Symposium in Dubrovnic, Croatia, June 2008. The mechanism of geneenvironment interactions in depression: Evidence that genes potentiate multiple sources of adversity.

Presentation on career opportunities and the life of a PhD student at the 'Biological career information day' of Maastricht University, September 2007 and September 2008.

Information evening on Mindfulness and Depression at the Community Mental Health Center (RIAGG), Maastricht, April 2008.

Presentation on Mindfulness and Depression at the Arcus Volksuniverseit in Heerlen, November 2008.

Presentation of Research Proposal 'Predictors of Successful Weight Loss' at the Netherlands Organisation for Scientific Research (NWO), 2007.

Poster Presentation at the British Feeding and Drinking Group (BFDG) Conference in Newcastle upon Tyne, 2007. Dietary restraint modulates the effects of food cue exposure on body and weight satisfaction.

\section{MEDIA}

L1 TV: Feature on Mindfulness; January 2009.

Depressies kunnen niet tegen klein geluk - Article in newspapers 'De Limburger' and 'Limburgs Dagblad'; December 2008.

Mindfulness tegen depressie - Article in magazine Palet; November 2008.

February 28th, 2008: Gedachten beschouwen als boten die voorbij varen - Article in University newspaper 'Observant'. 